\title{
Determinanten der Arbeitgeberwahl von potenziellen Bewerbern in der Ernährungsindustrie
}

\author{
Dissertation \\ zur Erlangung des Doktorgrades \\ der Fakultät für Agrarwissenschaften \\ der Georg-August-Universität Göttingen
}

vorgelegt von

Marina Abramovskij

geboren in Kiew (Ukraine)

Göttingen, im Juli 2013 
D 7

1. Referent: Prof. Dr. Ulrich Enneking

2. Korreferent: Prof. Dr. Rainer Marggraf

Tag der mündlichen Prüfung: 4. Juli 2013 


\section{Danksagung}

Die vorliegende Arbeit befasst sich mit dem Informations- und Entscheidungsverhalten potenzieller Bewerber in der Ernährungsindustrie. Zum erfolgreichen Gelingen dieser Arbeit haben verschiedene Personen einen sehr wertvollen Beitrag geleistet, für den ich mich an dieser Stelle herzlich bedanken möchte.

Mein außerordentlicher Dank gilt meinem Doktorvater, Prof. Dr. Ulrich Enneking, der mich mit seiner Aufgeschlossenheit für eine interdisziplinäre Zusammenarbeit, seinen kreativen Forschungsideen und seinen kritischen Fragen sehr unterstützt hat. Durch ihn habe ich gelernt, dass die Person-Vorgesetzten-Passung nicht nur ein theoretisches Konstrukt ist. Darüber hinaus möchte ich Prof. Dr. Rainer Marggraf für die Übernahme des Zweitgutachtens danken. Prof. Dr. Ludwig Theuvsen danke ich für die Mitwirkung in der Promotionskommission.

Danken will ich auch meinem Gymnasiallehrer Horst Rosemann, der bei mir das Interesse und die Freude am kritischen Diskurs geweckt hat. Prof. Dr. Karin Schnitker danke ich für ihre motivierenden Aufmunterungen, die mir über manches Hindernis hinweggeholfen haben.

Schließlich möchte ich mich ganz besonders herzlich bei meiner Familie und meinen Freunden bedanken: Meinen Eltern, weil ich mich immer und in allen Lebenslagen auf sie verlassen kann. Bei Leonardo Grani möchte ich mich für seine Geduld in allen Phasen der Promotion und seine unterstützend kritische Haltung bedanken. Helena Olfert danke ich schließlich für die vielen konstruktiven Gespräche „rund“ um die Promotion, die so manchen Zweifel an der Richtigkeit dieses Vorhabens ausräumen konnten.

Osnabrück, im Mai 2013 


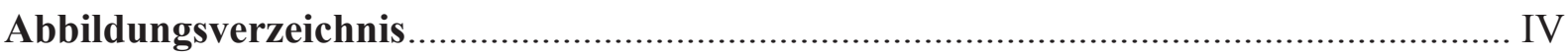

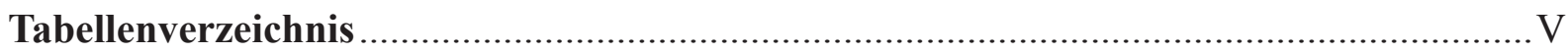

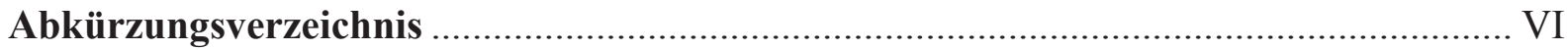

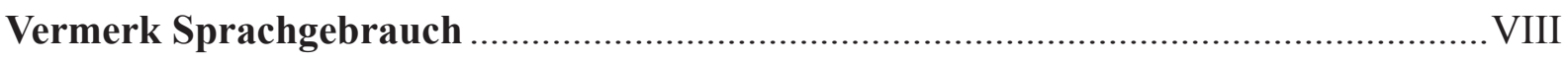

$1 \quad$ Einleitung

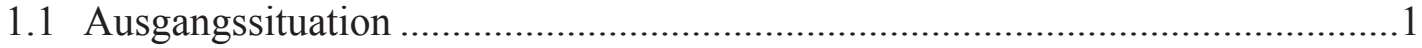

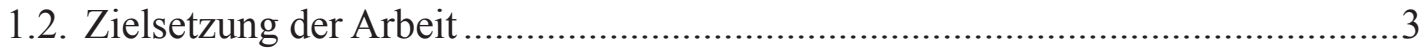

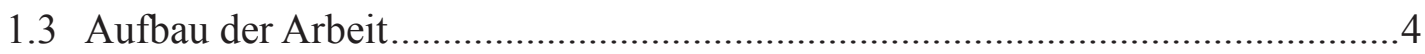

2 Konzeptionelle Grundlagen des Personalmarketings

2.1 Entstehung und Definition des Begriffs Personalmarketing .............................6

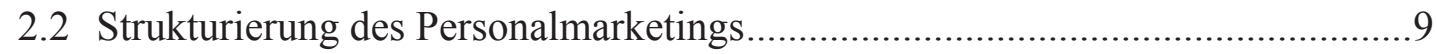

2.3 Instrumente der externen Bewerberansprache ..................................................12

2.4 Stellenwert des Personalmarketings innerhalb der Ernährungsindustrie ............15

2.5 Empirische Perspektive des Personalmarketings .............................................. 18

2.5.1 Wissenschaftliche Studien zum Personalmarketing ..............................18

2.5.2 Praxisorientierte Studien zum Personalmarketing ................................25

3 Theoretische Grundlagen der Arbeitgeberwahl .30

3.1 Determinanten der Arbeitgeberwahl aus informationsökonomischer Sicht.......31

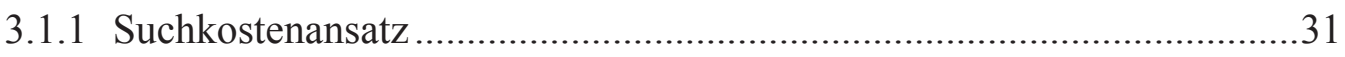

3.1.2 Informationsökonomische Eigenschaftstypologie ................................33

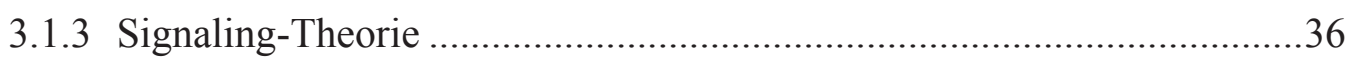

3.2 Determinanten der Arbeitgeberwahl aus erwartungswerttheoretischer Sicht ....41

3.3 Determinanten der Arbeitgeberwahl aus prozessualer Sicht.............................45

3.3.1 Prozessmodell von Soelberg ................................................................45

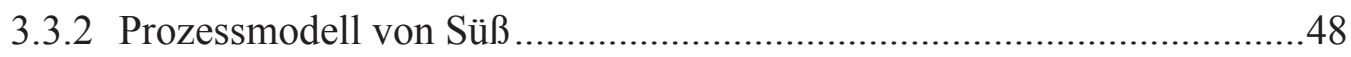

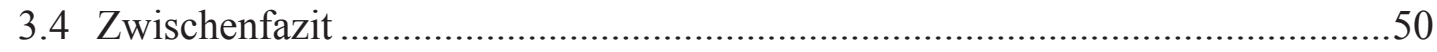

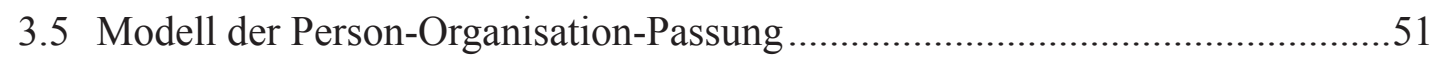

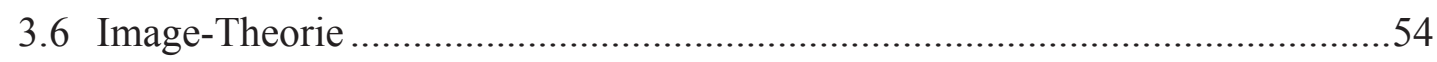

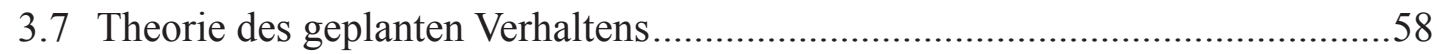

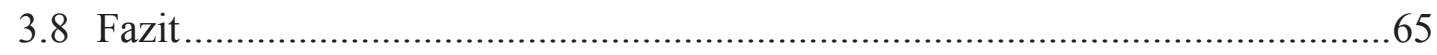

4.1 Informationsverhalten potenzieller Bewerber................................................67

4.2 Differenzielle Wirkung des Arbeitgeberimages .............................................69 


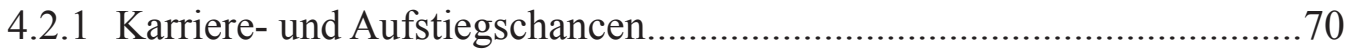

4.2.2 Vereinbarkeit von Beruf und Familie/Privateben .................................. 70

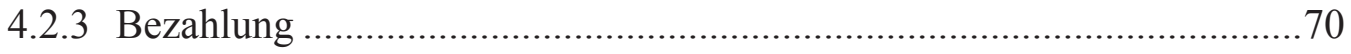

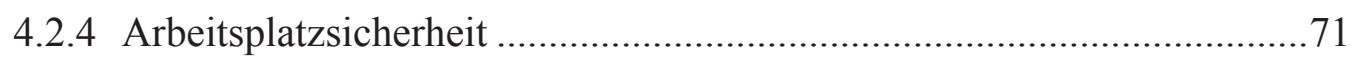

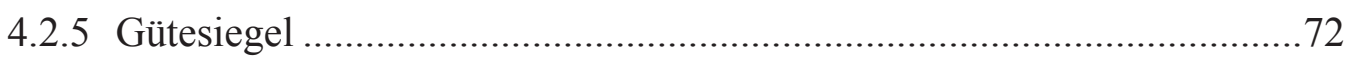

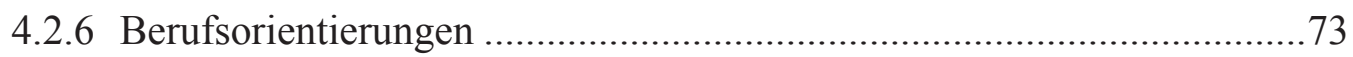

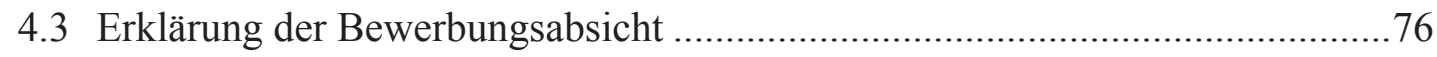

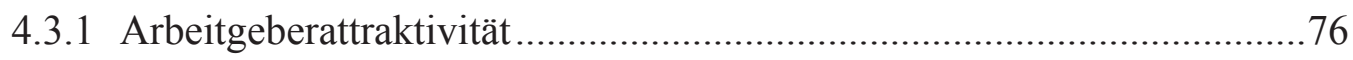

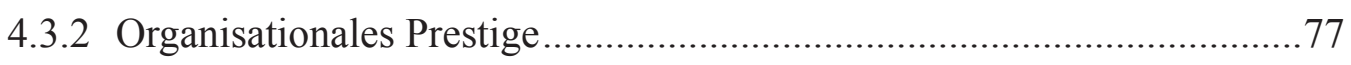

4.3.3 Erfolgswahrscheinlichkeit der Bewerbung ..........................................79

4.3.4 Alternativen auf dem Arbeitsmarkt..................................................... 79

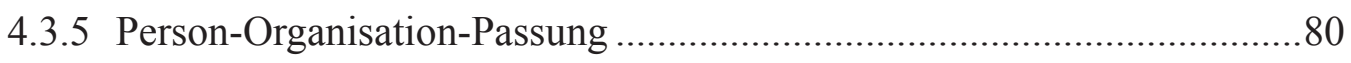

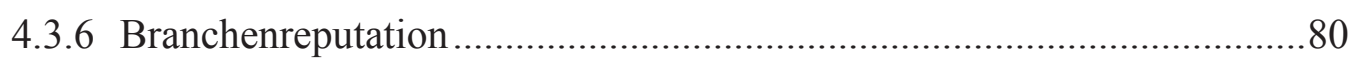

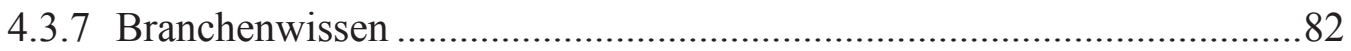

4.4 Zusammenfassende Darstellung der Untersuchungsmodelle und Hypothesen...83

5.1 Methodenwahl für die Messung des zweiten Teilmodells ................................8

5.2 Experimentelles Design der Discrete-Choice-Analyse ....................................92

5.3 Methodenwahl für die Messung des dritten Teilmodells ..................................95

5.4 Operationalisierung der Modellkonstrukte..................................................... 100

5.4.1 Operationalisierung des Arbeitgeberimages .......................................100

5.4.1.1 Operationalisierung der Karriere- und Aufstiegschancen .........101

5.4.1.2 Operationalisierung der Vereinbarkeit von Beruf und Familie...101

5.4.1.3 Operationalisierung der Bezahlung ..........................................101

5.4.1.4 Operationalisierung der Arbeitsplatzsicherheit ........................102

5.4.1.5 Operationalisierung der Gütesiegel ........................................102

5.4.2 Operationalisierung der Berufsorientierungen...................................... 103

5.4.3 Operationalisierung der Konstrukte zur Vorhersage

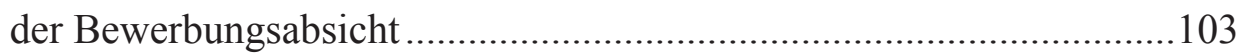

5.4.3.1 Operationalisierung der Arbeitgeberattraktivität ......................105

5.4.3.2 Operationalisierung des organisationalen Prestiges ..................105

5.4.3.3 Operationalisierung der Erfolgswahrscheinlichkeit

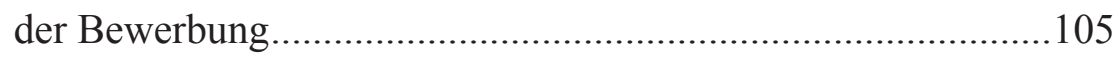

5.4.3.4 Operationalisierung der Bewerbungsabsicht ............................106

5.4.3.5 Operationalisierung der Person-Organisation-Passung .............106

5.4.3.6 Operationalisierung der Alternativen auf dem Arbeitsmarkt.....107

5.4.3.7 Operationalisierung der Branchenreputation.............................107

5.4.3.8 Operationalisierung des Branchenwissens ...............................107 
5.4.4 Operationalisierung der Methoden zur externen Bewerberansprache ...108

5.4.5 Operationalisierung des Involvements.............................................. 108

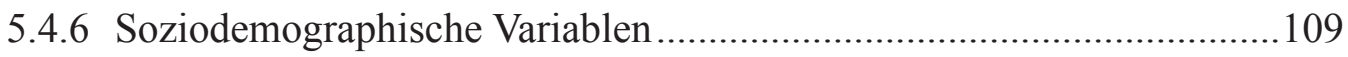

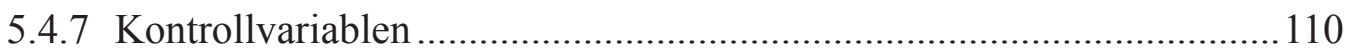

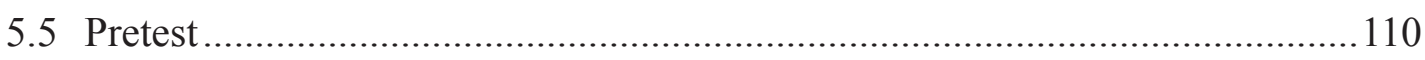

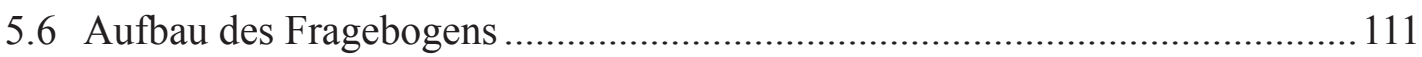

5.7 Durchführung der Befragung und Stichprobenauswahl ................................112

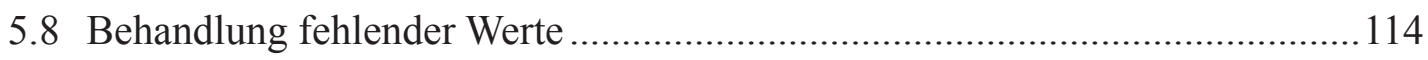

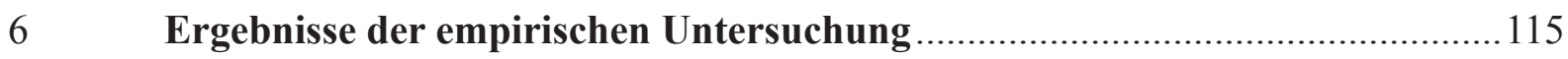

6.1 Beschreibung der Stichprobe ................................................................... 115

6.2 Analyse des ersten Teilmodells: Informationsverhalten potenzieller Bewerber.. 117

6.2.1 Bekanntheitsgrad der Kommunikationsmaßnahmen ...........................120

6.2.2 Nutzungsintensität der Kommunikationsmaßnahmen ..........................123

6.2.3 Nützlichkeitsbewertung der Kommunikationsmaßnahmen...................126

6.2.4 Präferenzmaß der Kommunikationsmaßnahmen..................................130

6.3 Analyse des zweiten Teilmodells:

Differenzielle Wirkung des Arbeitgeberimages ............................................131

6.4 Analyse des dritten Teilmodells: Erklärung der Bewerbungsintention.............140

6.4.1 Beurteilung des Messmodells ........................................................... 140

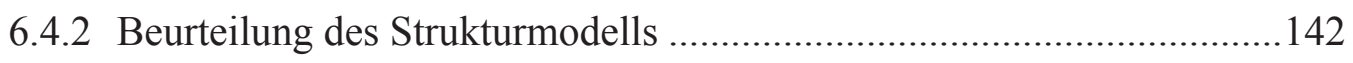

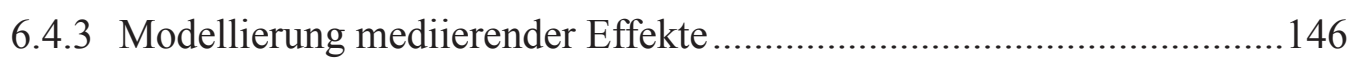

6.5 Zusammenfassende Darstellung der Hypothesenprüfung................................149

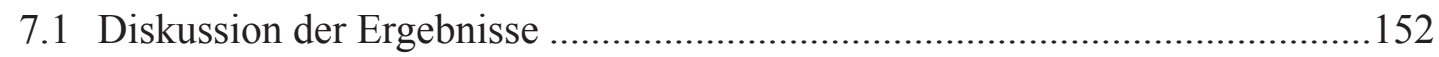

7.1.1 Ergebnisdiskussion zum ersten Teilmodell........................................152

7.1.2 Ergebnisdiskussion zum zweiten Teilmodell ......................................155

7.1.3 Ergebnisdiskussion zum dritten Teilmodell ........................................161

7.2 Limitationen und weiterer Forschungsbedarf ...............................................163

7.3 Implikationen für die Unternehmenspraxis................................................. 168

$8 \quad$ Zusammenfassung

Literaturverzeichnis 


\section{Abb.-Nr. Bezeichnung}

2-1 Personalmarketing als Prozess

2-2 Entwicklungsprognose des Erwerbspotenzials in Deutschland

3-1 Modell der Person-Organisation-Passung

3-2 Modell kognitiver Entscheidungsstrukturen eines potenziellen Bewerbers bei der Arbeitgeberwahl

3-3 Theorie des geplanten Verhaltens

4-1 Postulierte Zusammenhänge im ersten Teilmodell

4-2 Postulierte Zusammenhänge im zweiten Teilmodell

4-3 Postulierte Zusammenhänge im dritten Teilmodell

5-1 Vorgehen bei der DCA

5-2 Fragebogenaufbau

6-1 Bekanntheitsgrad der beiden Gütesiegeln

6-2 Eingeschätzte Relevanz der Gütesiegel bei der Arbeitgeberwahl

6-3 Parameterschätzungen des Kausalmodells nach Bootstrapping mit 200 Stichproben

6-4 Mediatorenmodell 


\section{Tab.-Nr. Bezeichnung}

2-1 Ausgewählte Instrumente des Personalmarketings

Anzahl von Unternehmen und Mitarbeitern in der Ernährungsindustrie mit 20 und mehr Beschäftigten im Jahr 2009 in Deutschland

2-3 Unternehmensgrößenabgrenzung des Instituts für Mittelstandsforschung (Eigene Darstellung)

2-4 Unternehmensgrößenabgrenzung der Europäischen Kommission

2-5 Struktur der produzierenden Ernährungsindustrie für Unternehmen mit 20 und mehr tätigen Personen

2-6 Anbieter von Arbeitgeberwettbewerbern, Zertifizierungen und Rankings

3-1 Typologie der Leistungseigenschaften zur Charakterisierung von Kaufentscheidungsprozessen

3-2 Such-, Erfahrungs- und Vertrauenseigenschaften eines Stellenangebotes

5-1 Unterschiede zwischen der TCA, der DCA und der Policy Capturing bzw. Vignettenanalyse

5-2 Methodenvergleich zwischen PLS und Kovarianzstrukturanalyse

5-3 Arbeitsplatzmerkmale und ihre Ausprägungen im Discrete-Choice-Modell

5-4 Deskriptive Statistiken und interne Konsistenzen (Cronbach's) aller im Teilmodell 3 berücksichtigten Skalen (N=671)

6-1 Sozidemographische Merkmale der Stichprobe

6-2 Bekanntheitsgrad, Nutzungsintensität, Nützlichkeitsbewertung und Präferenz einzelner Personalmarketingmaßnahmen

6-3 Logistische Regression zur Vorhersage des Bekanntheitsgrades von Maßnahmen

6-4 Logistische Regression zur Vorhersage der Nutzungsintensität von Maßnahmen

6-5 Schrittweise multiple Regression zur Vorhersage der Nützlichkeitsbewertung von Maßnahmen

6-6 Zusammenhänge von soziodemographischen Merkmalen mit dem Präferenzmaß von Maßnahmen

6-7 Modellvergleich anhand des AIC

6-8 Schätzergebnisse des Discrete-Choice-Modells

6-9 Güte der Messmodelle

6-10 Analyseergebnisse der mediierenden Effekte

6-11 Zusammenfassende Darstellung der Hypothesenprüfung 
Abb. Abbildung

AEW Agrar- und Ernährungswissenschaften

AIC Akaike-Informationskriterium

AVE durchschnittlich erfasste Varianz

BWL Betriebswirtschaftslehre

bzw. beziehungsweise

ca. circa

CA Conjoint-Analyse

CSR Corporate Social Responsibility

DCA Discrete-Choice-Analyse

DCE Discrete-Choice-Experiment

ELM Elaborations-Wahrscheinlichkeits-Modell

et al. et alii

EU Europäische Union

f. folgende

FR Faktorreliabilität

ggf. gegebenenfalls

Hrsg. Herausgeber

i.d.R. in der Regel

IfM Institut für Mittelstandforschung Bonn

IIA independence of irrelevant alternatives

KMU kleine und mittlere Unternehmen

LL LogLikelihood

LMT Lebensmitteltechnologie

LR Likelihood-Ratio

Mio. Millionen

ML Maximum-Likelihood-Schätzung

MNL Multinomiales Logit-Modell

Nr. Nummer

o.J. ohne Jahr

OR Odds Ratio

PLS Partial-Least-Squares

sog. sogenannte

TCA traditionelle Conjoint-Analyse

Tab. Tabelle

TOPB theory of planned behavior

Tsd. Tausend

u.a. unter anderem

vgl. vergleiche

VIF Variance Inflation Factor 
vs. versus

z.B. zum Beispiel

zit. zitiert

z.T. zum Teil 


\section{Zum Sprachgebrauch in dieser Arbeit}

Vorab wird betont, dass in dieser Arbeit Personen männlichen und weiblichen Geschlechts gemeint sind. Wenn in dieser Arbeit zur Vereinfachung und besseren Lesbarkeit ausschließlich die kürzere männliche Form benutzt wird, so sind ausdrücklich weibliche Personen eingeschlossen. 
KAPITEL 1

\section{Einleitung}

\subsection{Ausgangssituation}

Mitarbeiter gelten nach dem Verständnis des Human Resource Managements als das wichtigste Kapital eines Unternehmens (ARMSTRONG, 2012). Zu diesem wissenschaftlichen Grundsatz bekennen sich heute fast alle Unternehmen in ihren Leitbildern oder Grundsätzen (SPONHEUER, 2010). Eine dauerhafte Gewinnung von Mitarbeitern ist infolgedessen als entscheidender Wettbewerbsfaktor zu sehen, der sich inzwischen auch empirisch hinreichend nachweisen lässt (für Beispiele vgl. MARCUS, 2011). Für die stetig zunehmende Bedeutung des Personalmarketings innerhalb des Personalmanagements lassen sich zugleich weitere Ursachen identifizieren. So wird in verschiedenen Studien aufgrund des demographischen Wandels mittelfristig eine immense Verknappung von verfügbaren Arbeitnehmern prognostiziert, was wiederum zu erhöhten Akquisitionsanstrengungen zur Deckung des bestehenden qualitativen und quantitativen Personalbedarfs führt (KAY et al., 2010). Hierdurch wandeln sich viele Bereiche des Arbeitsmarktes von einem Arbeitgeber- hin zu einem Arbeitnehmermarkt (BÖTTGER, 2012). Neben dem demographisch verursachten Mangel an Fach- und Führungskräften gewinnt das Personalmarketing auch aufgrund des Wertewandels, des zunehmend global ausgetragenen und deshalb verschärften Wettbewerb um qualifizierte Arbeitskräfte, der stetig wachsenden Bedeutung von wissens- und dienstleistungsintensiven Unternehmensaktivitäten sowie der steigenden qualitativen Anforderungen der Unternehmen an ihre aktuellen und potenziellen Mitarbeiter immer mehr an Aufmerksamkeit (KIRCHGEORG und MÜLLER, 2011).

Die Verfügbarkeit von Fach- und Führungskräften stellt derzeit auch in der Ernährungsindustrie derzeit ein zentrales Branchenthema dar. Das branchenspezifische Ausmaß dieser Problematik lässt sich beispielsweise anhand einer Studie von THEUVSEN et al. (2009) konkretisieren, in der 51 Prozent der befragten Unternehmen angaben, dem Thema einen hohen Stellenwert beizumessen. Auch die Ergebnisse einer Studie des Beratungsunternehmens AFC verdeutlichen, dass es sich dabei um einen gewichtigen Problembereich handelt (ELLES et al., 2012). Dort gab immerhin rund ein Drittel der befragten Unternehmen an, dass sie die zukünftige Anwerbung von Akademikern für gefährdet halten. 
Für die überwiegend mittelständischen Unternehmen der Ernährungsindustrie kommt bei der Deckung ihres Personalbedarfs allerdings erschwerend hinzu, dass sie im Gegensatz zu Großunternehmen oftmals kein ausgeprägtes und effektiv kommuniziertes Arbeitgeberimage aufweisen und auch aufgrund ihrer zumeist auf die Region beschränkten Bekanntheit Probleme haben, die notwendigen qualifizierten Fach- und Führungskräfte zu rekrutieren (SCHMIDTKE, 2001). Darüber hinaus dürften auch mangelnde Branchenkenntnisse seitens potenzieller Bewerber und ein vergleichsweise schlechtes Reputationsniveau der Ernährungsindustrie die Gewinnung von geeigneten Mitarbeitern spürbar beeinträchtigen (ALBERSMEIER und SPILLER, 2009).

$\mathrm{Zu}$ der grundsätzlichen Frage der Mitarbeitergewinnung liegt bereits eine Vielzahl wissenschaftlicher und praxisorientierter Studien vor, die zumeist auf einer bewerberseitigen Betrachtungsweise basieren. Beschäftigt man sich mit der Fülle der berichteten Ergebnisse allerdings etwas genauer, so wird sehr schnell deutlich, dass ihr Beitrag zur Lösung der oben skizzierten Problemlage sowohl aus der Forschungs- als auch aus der Unternehmensperspektive zumindest bislang als insgesamt gering zu werten ist. Als ursächlich hierfür lassen sich mehrere Aspekte benennen. So fehlt in den bisherigen Studien zum einen eine speziell auf die Ernährungsindustrie ausgerichtete Analyse des Informations- und Entscheidungsverhaltens potenzieller Bewerber, obgleich bereits deutliche Hinweise für die Relevanz branchenspezifischer Determinanten, wie beispielsweise der Branchereputation, vorliegen (HAUTZINGER, 2009). In diesem Kontext wurde bislang auch nicht untersucht, ob und inwieweit sich die von den Unternehmen eingeschlagenen Rekrutierungswege bei den für sie relevanten Zielgruppen als wirksam erweisen. Ferner ist generell zu konstatieren, dass die Art, wie Entscheidungen von Bewerbern tatsächlich zustande kommen, bisher nur in Ansätzen erforscht ist (vgl. BoswELL et al., 2012). Kritisch muss ferner zur derzeitigen Forschungslage angemerkt werden, dass die in den beiden hier besonders relevanten Disziplinen - der marketingwissenschaftlichen und der psychologischen Forschung - erzielten Erkenntnisse nur unzureichend miteinander verknüpft wurden. Ein weiterer Problempunkt ist darin zu sehen, dass die Erhebung unmittelbarer Wichtigkeitsurteile von Arbeitsplatzmerkmalen, wie es in den bisherigen Studien überwiegend der Fall war, wenig geeignet ist, die Präferenzen potenzieller Bewerber valide zu messen (MOSER und ZEMPEL, 2006). Ebenso fehlt es derzeit an wissenschaftlichen Studien, die sich mit den neueren Entwicklungen des Personalmarketings, wie dem stetig wachsenden Einsatz von Gütesiegeln, fundiert auseinandersetzen (vgl. SCHERM und SÜSS, 2010; NAUNDORF und SPENGLER, 2012). Insgesamt ergibt sich hieraus die wesentliche Forderung, schlüssige Modelle zu entwickeln, die es erlauben, die bislang unberücksichtigten Phänomene mithilfe interdisziplinärer Forschungsansätze zu analysieren und zu erklären und dadurch eine derzeit noch vorhandene Forschungslücke zu schließen. 


\subsection{Zielsetzung der Arbeit}

Wie die vorangegangenen Ausführungen zeigen, liegen bislang keine hinreichenden Erkenntnisse zum Informations- und Entscheidungsverhalten potenzieller Bewerber in der Ernährungsindustrie vor, obgleich eine entsprechende branchenspezifische Analyse sowohl aus der Unternehmens- als auch aus der Bewerbersicht sehr bedeutsam ist. Eine differenzierte und fundierte Kenntnis der bevorzugten Informationsquellen, relevanten Bewertungskriterien und Indikatoren, die eine Bewerbungsentscheidung begünstigen, eröffnet mittelständischen Unternehmen aus der Ernährungsindustrie die Möglichkeit, ihre Rekrutierungsaktivitäten optimal auf die relevante Zielgruppe auszurichten und nicht zuletzt dadurch besonders qualifizierte Bewerber für das eigene Unternehmen zu gewinnen. Auf der anderen Seite sollte die Kenntnis der persönlichen beruflichen Ziele und ihrer Auswirkungen auf die Arbeitgeberwahl potenziellen Bewerbern dazu verhelfen, ihre eigene Bewerbungsentscheidung transparenter und damit nachhaltiger fällen zu können.

Vor dem Hintergrund der oben aufgezeigten konzeptionellen und methodischen Lücken in der bisherigen Forschung zur Arbeitgeberwahl, ist das zentrale Ziel dieser Arbeit darin zu sehen, das Informations- und Entscheidungsverhalten potenzieller Bewerber in der Ernährungsindustrie einer fundierten und dabei möglichst differenzierten Analyse zu unterziehen. Hiervon ausgehend lassen sich drei wissenschaftliche Teilziele formulieren. So wird zum einen untersucht, welche Zusammenhänge zwischen verschiedenen soziodemographischen und persönlichen Merkmalen der Befragten und ausgewählten Maßnahmen des externen Personalmarketings bestehen. Ausgehend von einem mehrstufigen Personalmarketingprozess soll damit zugleich die in der ersten Phase vorherrschende Frage beantwortet werden, welche Rekrutierungskanäle dafür ausschlaggebend sind, dass aus potenziellen Bewerbern tatsächliche Bewerber werden.

Weiterhin steht die Frage im Vordergrund, was potenziellen Bewerbern wichtig ist, wenn sie sich für ein konkretes Stellenangebot zu entscheiden haben. Neben der Bedeutung ausgewählter Arbeitsplatzmerkmale wird hierbei der Einfluss von Gütesiegeln untersucht. $\mathrm{Ob}$ sich ein Gütesiegel auf die Wahl eines Stellenangebotes auswirkt, sollte einerseits von seiner Bekanntheit und grundsätzlichen Relevanz für potenzielle Bewerber abhängen. Andererseits ist es naheliegend anzunehmen, dass sich seine Signalfunktion besonders dann entfalten sollte, wenn die Botschaft des Gütesiegels mit den Berufsorientierungen potenzieller Bewerber übereinstimmt. Aus methodischer Perspektive kommt bei diesem Teilmodell die Überlegung hinzu, dass ein marketingwissenschaftliches Verständnis eines Stellenangebotes als Produkt, das gegenüber Kunden, in diesem Fall potenziellen Bewerbern, möglichst attraktiv zu gestalten ist, auch eine entsprechende Analysemethode erfordert. In der Anwendung eines dekompositionellen Präferenzmessverfahrens, der Discrete-Choice-Analyse, auf das Entscheidungsverhalten potenzieller Bewerber liegt folglich ein weiterer Schwerpunkt dieser Arbeit. 
Ferner soll die wesentliche Forschungsfrage, wie es zu einer Bewerbungsentscheidung bei einem mittelständischen Unternehmen der Ernährungsindustrie kommt, mithilfe eines theoretischen Kausalmodells beantwortet werden. Dazu werden die informationsökonomischen und prozessualen Modelle der Arbeitgeberwahl zunächst als Diskussionsgrundlage herangezogen. Darüber hinaus wird die Anwendbarkeit der Theorie des geplanten Verhaltens als Rahmentheorie zur Vorhersage der Bewerbungsintention erörtert und dessen Modellkomponenten sowie postulierte Wirkbeziehungen auf die zugrunde liegende Fragestellung übertragen. Basierend auf diesem Grundmodell werden weitere theoriegeleitet ausgewählte Determinanten in das Kausalmodell integriert, die ihrerseits in psychologischen und marketingwissenschaftlichen Ansätzen zu verorten sind. So werden neben dem Modell der Person-Organisation-Passung die branchenspezifischen Determinanten in Form von Branchenwissen und Branchenruf berücksichtigt. Das erweiterte Kausalmodell dient als Grundlage der empirischen Untersuchung.

Aus den hier erzielten Ergebnissen sollen schließlich Handlungsempfehlungen für die Unternehmenspraxis abgeleitet werden, mit deren Hilfe sich zielgruppen- und branchenspezifische Personalmarketingaktivitäten strategisch planen und umsetzen lassen.

\subsection{Aufbau der Arbeit}

Die Arbeit gliedert sich in drei inhaltliche Komplexe: Während im ersten Abschnitt die theoretischen Grundlagen, Instrumente und empirische Studien des Personalmarketings erläutert werden, beinhaltet der zweite Anschnitt eine ausführliche Darstellung theoretischer Ansätze zum Entscheidungsverhalten potenzieller Bewerber. Im dritten Abschnitt werden darauf aufbauend drei Teilmodelle konzipiert und anschließend in einer empirischen Untersuchung überprüft.

Nach der Einleitung im ersten Kapitel folgt im zweiten Kapitel eine theoretische Erörterung der konzeptionellen Hintergründe des Personalmarketings. Neben einer definitorischen Abgrenzung werden dabei zunächst die dazugehörigen Instrumente und branchenspezifische Handlungsfelder aufgezeigt. Daran schließt sich eine systematische Analyse des aktuellen Forschungsstandes an, die sowohl aus einer wissenschaftlichen als auch praxisorientierten Perspektive erfolgt.

Das dritte Kapitel widmet sich einer ausführlichen Betrachtung von informationsökonomischen und prozessorientierten Ansätzen der Arbeitgeberwahl. Darauffolgend werden die theoretischen Grundlagen des Modells der Person-Organisation-Passung, der Image-Theorie sowie der Theorie des geplanten Verhaltens erläutert. 
Aufbauend auf den theoretischen Erkenntnissen und den daraus resultierenden relevanten Konstrukten werden drei Teilmodelle zur umfassenden Analyse des Informations- und Entscheidungsverhaltens potenzieller Bewerber konzipiert. Im ersten Teilmodell wird der Forschungsfrage nachgegangen, ob und inwieweit das Informationsverhalten potenzieller Bewerber mit ihren soziodemographischen und persönlichkeitsspezifischen Merkmale zusammenhängt. Die Bedeutung ausgewählter Arbeitsplatzmerkmale steht im Fokus des zweiten Teilmodells. Basierend auf den Annahmen der Theorie des geplanten Verhaltens wird im dritten Teilmodell überprüft, welche Determinanten für die Entstehung einer Bewerbungsabsicht ausschlaggebend sind. Die in den Teilmodellen postulierten Wirkbeziehungen werden dabei anhand des jeweiligen Hypothesensystems abgebildet, die ihrerseits als Grundlage der empirischen Untersuchung dienen.

Der folgende empirische Teil - beginnend mit dem Kapitel fünf - befasst sich zunächst ausführlich mit den verwendeten Mess- und Analyseverfahren sowie den dabei eingesetzten Instrumenten und dem zugrunde liegenden Datensatz. Eine Besonderheit dieser Arbeit stellt zugleich das breite methodische Instrumentarium dar, auf das innerhalb der statistischen Analyse der drei Teilmodelle zurückgegriffen wird. In diesem Kontext erfährt auch die Operationalisierung der theoretischen Konstrukte eine ausführliche Betrachtung.

Im daran anschließenden Kapitel fünf werden die empirischen Untersuchungsergebnisse der drei Teilmodelle detailliert vorgestellt. Dabei wird anhand der erzielten Ergebnisse jeweils überprüft, ob die entsprechenden Forschungshypothesen zu bestätigen oder aber zu verwerfen sind. Den Schluss dieses Kapitels bildet eine überblicksartige Betrachtung aller theoretischen Hypothesen und der dazugehörigen empirischen Befunde.

Das letzte siebte Kapitel dieser Arbeit setzt sich kritisch mit den empirischen Befunden auseinander und zeigt neben den daraus gewonnen Erkenntnissen auch ihre Beschränkungen und den damit einhergehenden weiterführenden Forschungsbedarf auf. Die wissenschaftliche Diskussion wird um eine praxisorientierte Beurteilung der Ergebnisse samt gezielten Handlungsempfehlungen für mittelständische Unternehmen der Ernährungsindustrie ergänzt. Eine zusammenfassende Betrachtung bildet den Abschluss dieser Arbeit. 


\section{Konzeptionelle Grundlagen des Personalmarketings}

\subsection{Entstehung und Definition des Begriffs Personalmarketing}

Die wissenschaftliche Anwendung von Marketing-Konzepten auf Fragen der Personalbeschaffung und -entwicklung reicht bis in die späten 60er Jahre zurück (FRANKE, 2000; MOSER und ZEMPEL, 2006). Im Zuge des hohen bundesdeutschen Wirtschaftswachstums in den Jahren 1968 bis 1975 und der dadurch bedingten zunehmenden Knappheit an qualifizierten Arbeitskräften verbreitete sich das Konzept des Personalmarketings in der deutschsprachigen Fachliteratur. Nach 1975 lässt sich allerdings ein deutlicher Rückgang des Interesses an Personalmarketingfragen verzeichnen, was auf die damals aufkommende Massenarbeitslosigkeit zurückgeführt wird. Seit den 80er Jahren steigt die Zahl der wissenschaftlichen Arbeiten zum Personalmarketing wieder an (KIRCHGEORG und MÜLLER, 2011).

Als eine der ersten wissenschaftlichen Konzeptionen des Personalmarketings wird die Arbeit von HUNZIKER (1973) gekennzeichnet, in der es als eine Subfunktion des Personalmanagements betrachtet wird und somit lediglich einige Teilbereiche, vor allem die Personalbeschaffung und die Personalwerbung, umfasst. Die nächsten Schritte in der Entwicklung des Personalmarketings bestanden sodann in der „langfristig angelegten mitarbeiterorientierten Personalpolitik“ (FRÖHLICH, 1987: 43). Demzufolge dient der Arbeitsplatz als mögliches Mittel zur Erfüllung menschlicher Bedürfnisse, die u.a. im Bestreben nach Partizipation an organisationalen Entscheidungsprozessen zu lokalisieren sind. Seine größte Begriffsfassung erfährt das Personalmarketing innerhalb des Unternehmensführungs-Ansatzes. Darin wird es als die „Orientierung der gesamten Personalpolitik eines Unternehmens an den Bedürfnissen von gegenwärtigen und zukünftigen Mitarbeitern, mit dem Ziel, gegenwärtige Mitarbeiter zu motivieren und neue Mitarbeiter zu gewinnen“ beschrieben (SIMON et al., 1995: 13). Im Vordergrund steht somit die Überlegung, sämtliche Unternehmensaktivitäten konsequent an den Vorstellungen und Bedürfnissen der vorhandenen und zukünftigen Mitarbeiter auszurichten. 
Der nächste Schritt in der theoriebasierten Entwicklung des Personalmarketings erfolgt mit dem Konzept des Generic Marketings, worin die aus dem klassischen Konsumgütermarketing bekannten Theorien, Konzepte und Instrumente auf die bestehenden Aufgaben im Personalbereich übertragen werden (KOTLER, 1972). Die zugrunde liegende Kernannahme besteht hierbei darin, dass Austauschprozesse nicht nur in klassischen Anwendungsbereichen des Marketings stattfinden. Vielmehr wird eine Ausweitung des Marketinggedankens auf sämtliche Organisations- und Steuerungseinheiten von Austauschprozessen an Märkten postuliert. Eine dieser Übertragungen stellt das Personalmarketing dar, sodass unter analoger Verwendung von Marketingstrategien und -instrumenten ein Austausch von Arbeitsangebot und -nachfrage vollzogen werden kann. Auf diesem Marketingverständnis basierend lässt sich das Personalmarketing als ein organisationaler Prozess kennzeichnen, das darauf abzielt, das Produkt Arbeitsplatz bzw. Stellenangebot möglichst attraktiv zu gestalten ist, um Kunden in Form potenzieller und tatsächlicher Mitarbeiter für die eigene Organisation zu gewinnen bzw. sie langfristig an diese zu binden (SCHOLZ, 2000).

In der Literatur wird der Begriff, Aufgabenbereich und Zielgruppenbezug des Personalmarketings unterschiedlich weit gefasst und reicht von der reinen Gewinnung potenzieller Mitarbeiter bis zu einem ganzheitlichen Verständnis, in dem der Zielgruppenbezug um tatsächliche Mitarbeiter erweitert wird (vgl. für einen Überblick: FRANKE, 2000). Letzterem zufolge lässt sich das Personalmarketing als ein „Prozess charakterisieren, der bei der Ansprache potenzieller Bewerber beginnt und bis zu Bemühungen um den dauerhaften Verbleib neuer Mitarbeiter in der Organisation reicht“" (MOSER und ZEMPEL, 2006: 70). Mit dieser Auffassung rücken zugleich die Bedürfnisse und Erwartungen sowohl potenzieller als auch aktueller Mitarbeiter in den Fokus des Personalmarketings (KIRCHGEORG und MüLLER, 2011). MOSER und ZEMPEL (2006) werten eine solch breit angelegte Perspektive jedoch als problematisch, da insbesondere deutschsprachige Publikationen nahezu sämtliche Maßnahmen des Personalmanagements auch als Bestandteile des Personalmarketings behandeln. In der Unternehmenspraxis überwiegt gleichwohl die Bedeutung des Personalmarketings im engeren Sinne, da sich die zugrunde liegenden Aktivitäten auf die Bewerberansprache und Rekrutierung konzentrieren. Vor diesem Hintergrund bildet dieses Aufgabenfeld den Schwerpunkt der folgenden Ausführungen.

Ein relativ neuer Ansatz im Forschungsfeld des Personalmarketings besteht im sog. Employer Branding, worunter der strategisch gesteuerte Auftritt eines Unternehmens als attraktiver Arbeitgeber verstanden wird (BÖTTGER, 2012). Bei diesem Ansatz werden die ursprünglich auf Konsumenten zielenden Bemühungen der Markenführung, ein Unternehmen bzw. seine Produkte und Dienstleistungen als besonders attraktiv zu positionieren und dadurch Nachfrage zu generieren, auf den Arbeitsmarkt übertragen (SPONHEUER, 2010). Die zentrale Annahme besteht hierbei darin, dass die Verbesserung der eigenen Arbeitgeberattraktivität auch die Anzahl geeigneter Bewerber, die hinsichtlich ihrer Wertvorstellungen und Anforderungen besonders gut zur Organisation passen, steigert (KIRCHGEORG und MÜLLER, 2011). Allerdings 
liegt ein allgemein anerkanntes und theoretisch fundiertes Verständnis des Employer Branding Ansatzes bislang noch nicht vor (SPONHEUER, 2010). Das Konzept stützt sich derzeit vielmehr auf praxisnahe Veröffentlichungen, die ihrerseits zumeist auf die Vermarktung eigener Angebote von Unternehmens- und Personalberatungen sowie Werbeagenturen abzielen (GROBE, 2008). Gleichwohl steigt die Anzahl von konzeptionellen und empirischen Forschungsarbeiten in diesem Bereich in der letzten Zeit bemerkenswert an, sodass von einer baldigen Etablierung dieses Forschungsansatzes in der Marketingwissenschaft auszugehen ist (EDWARDS, 2010).

Wenngleich der Begriff des Personalmarketings in der Forschung und Praxis etabliert ist, wird er von einigen Autoren hinsichtlich seiner semantischen und auch inhaltlichen Konzeption kritisch hinterfragt (FRANKE, 2000). So bemängeln MOSER und ZEMPEL (2006), dass mit dem Begriff per se eine irreführende semantische Wirkung einhergeht, da hierbei Arbeitsplätze und nicht Personen vermarktet werden. Darüber hinaus sehen die Autoren den Marketingbegriff grundsätzlich mit strategischen und kalkulativen Elementen im Umgang mit anderen Menschen verbunden, wodurch eine geminderte Aufrichtigkeit bzw. Authentizität wahrnehmbar wird. Die inhaltlichen Einwände beziehen sich indes auf die mit dem Personalmarketing einhergehende Analogie zum Absatzmarketing (MOSER, 1992). So erfordert die Übertragbarkeit von Marketingkonzepten auf den Personalbereich eine grundsätzliche Vergleichbarkeit von Marktstrukturen und Entscheidungsverhalten, die nicht einschränkungsfrei vorliegt. Ursächlich dafür ist zum einen, dass das Entscheidungsverhalten innerhalb der Arbeitsplatzwahl von anderen Kaufentscheidungsprozessen abweicht. So sind die Entscheidungen, die im Rahmen der Arbeitsplatzwahl stattfinden, relativ selten, sodass Lernmöglichkeiten aus Fehlentscheidungen eingeschränkt sind. Überdies steht beim Abschluss des Arbeitsvertrags die zu erbringende Leistung im Detail nicht fest, was ein gewisses Mindestmaß an beiderseitigem Vertrauen voraussetzt, was allerdings nicht in gleichem Maße für andere Kaufentscheidungen gilt. Schließlich liegt die Freiwilligkeit der Marktteilnahme aufgrund ökonomischer Zwänge aufseiten des Arbeitnehmers streng genommen häufig nicht vor. SÜSS (1996) verweist zudem auf die starke Regulierung des Arbeitsmarktes, beispielsweise durch Betriebsverfassungs- und Kündigungsschutzgesetz, Arbeitszeitverordnung sowie eine Vielzahl weiterer Kollektivvereinbarungen wie Tarifverträgen. Aus den dargestellten Differenzen zwischen Produktmärkten und dem Arbeitsmarkt schlussfolgert der Autor, dass im Bereich des Personalmarketings ein vergleichsweise geringer unternehmerischer Gestaltungsspielraum besteht. MALMENDIER (2006) führt hingegen an, dass die strukturellen Differenzen zwischen dem Arbeits- und Produkt- bzw. Dienstleistungsmarkt zwar stets eine Anpassung erfordern, eine generelle Übertragung von Marketingkonzepten und Instrumenten jedoch nicht wesentlich einschränken. So identifiziert der Autor beispielsweise im Bereich von privaten Rentenversicherungen ein Beispiel für einen Absatzmarkt, der mit dem Arbeitsmarkt vergleichbare Strukturen aufweist. Ferner führt er an, dass Hochschulabsolventen aufgrund ihrer hohen Qualifikation häufig außertarifliche Regelungen, z.B. übertarifliches Entgelt oder flexible Arbeitszeiten, vereinbaren. 


\subsection{Strukturierung des Personalmarketings}

KIRCHGEORG und MÜLlER (2011) nennen drei zentrale Aufgaben, die innerhalb des Personalmanagements originär dem Personalmarketing zugeschrieben werden. Dazu gehört erstens eine Profilierungsfunktion, die sich aus einem zukünftig schärferen Wettbewerb von Unternehmen und anderen Organisationen um besonders geeignete Mitarbeiter heraus begründet. Dabei werden solche Wettbewerbsvorteile angestrebt, mit denen die relevanten Zielgruppen am besten angezogen werden können. Zweitens dient die Akquisitionsfunktion dazu, bei potenziellen Bewerbern einen hohen Bekanntheitsgrad, eine Präferenz und Bewerbungsabsicht zu bewirken. Mithilfe der Motivationsfunktion soll schließlich der Arbeitsplatz und das Arbeitsumfeld tatsächlicher Mitarbeiter bedürfnisgerecht gestaltet werden, um sie möglichst lange an das Unternehmen zu binden. Das Personalmarketing wird dabei als die organisationale Einheit betrachtet, die sich gezielt den beschriebenen Funktionen widmet. Vor diesem Hintergrund erweist sich Personalmarketing als ein Prozess, der bei der Anwerbung qualifizierter Bewerber beginnt und sich über die Gestaltung des Auswahlverfahrens und die Festlegung der Konditionen eines Arbeitsvertrags bis hin zur Führung und langfristigen Personalentwicklung der Mitarbeiter fortsetzt (KANNING et al., 2009). Eine systematische Strukturierung des Personalmarketings lässt sich anhand von zwei Kategorien vornehmen (SIMON et al., 1995; MALMENDIER, 2006):

- Zielgruppe (extern vs. intern)

- Horizont (strategisch vs. taktisch)

Da im Rahmen von Stellenbesetzungen grundsätzlich zwei Wirkungsrichtungen möglich sind, nämlich die Ansprache externer Bewerber und der bereits in der Organisation tätigen Mitarbeiter, wird innerhalb der Personalmarketing-Konzeption zwischen einer internen und einer externen Orientierung unterschieden (SÜSS, 1996). Externes Personalmarketing umfasst folglich alle Ziele und Maßnahmen, die auf den externen Arbeitsmarkt wirken. Demgegenüber gehören alle nach innen gerichteten Maßnahmen zum internen Personalmarketing, das primär auf eine mitarbeiterorientierte Personalpolitik abzielt. Allerdings sind die aufgezeigten Wirkungsrichtungen aufgrund bestehender Interdependenzen nicht unabhängig voneinander zu betrachten. So kann beispielsweise eine inadäquate innerbetriebliche Führungspraxis und die damit einhergehende hohe Fluktuationsrate negative Auswirkungen auf das Arbeitgeberimage haben und damit die Personalbeschaffung nachhaltig beeinträchtigen (TEUFER, 1999).

Eine weitere Kategorisierungsebene stellt die Unterscheidung zwischen einer strategischen und einer taktischen Ausrichtung des Personalmarketings dar. Strategische Ansätze beinhalten langfristige betriebliche Grundsatzentscheidungen wie die Zielfestlegung für das Personalmarketing, die Zielgruppenselektion sowie die Positionierung der Organisation auf dem Arbeitsmarkt (SÜSS, 1996). Ausgangsbasis des strategischen Personalmarketings stellt der ermittelte quali- 
tative und quantitative Personalbedarf einer Organisation dar. Darauf aufbauend wird anhand verschiedener Kriterien, wie beispielsweise Ausbildungsniveau, Berufserfahrung und Art des angestrebten Beschäftigungsverhältnisses die angestrebte Zielgruppe auf dem Arbeitsmarkt bestimmt. An die Definition der relevanten Arbeitsmarktsegmente schließt sich eine detaillierte Analyse von zielgruppenspezifischen Bedürfnissen, Anforderungen und Erwartungen an. Eine genaue Kenntnis der relevanten Zielgruppe ist deshalb so bedeutsam, da sich potenzielle Bewerber hinsichtlich ihrer Erwartungen und Anforderungen an zukünftige Arbeitgeber deutlich voneinander unterscheiden (BÖTTGER, 2012). Mithilfe unterschiedlicher Segmentierungsansätze, die beispielsweise auf persönlichkeitsspezifischen oder soziodemographischen Merkmalen beruhen, kann die relevante Zielgruppe in mehrere homogene Cluster untergliedert werden, in denen wiederum die dafür jeweils besonders geeigneten Instrumente des Personalmarketings eingesetzt werden (MEFFERT et al., 2012). Gemäß dieser strategischen Ausrichtung wird das Personalmarketing folglich als ein langfristiger Prozess zur systematischen Schaffung von Präferenzen bei aktuellen und potenziellen Mitarbeitern gegenüber einer Organisation verstanden, der eines konsequenten und langfristigen Einsatzes von Personalmarketinginstrumenten bedarf (KIRCHGEORG und MÜLLER, 2011).

Demgegenüber ist die taktische Dimension des Personalmarketings darauf ausgerichtet, den Einsatz von Personalmarketinginstrumenten unter der Berücksichtigung der strategischen Ausrichtung zu planen und operativ umzusetzen. Obgleich über die Zusammensetzung eines optimalen, an den Bewerberbedürfnissen orientierten Personalmarketing-Mix in der marketingwissenschaftlichen Literatur bisher kein Konsens herrscht, wird in Anlehnung an den Strukturierungsvorschlag von SIMON et al. (1995) grundsätzlich zwischen der Leistungs-, Gehaltsund Kommunikationspolitik unterschieden.

Die Leistungspolitik bezieht sich im Wesentlichen auf die zielgruppengerechte Arbeitsplatzgestaltung. Hierbei nehmen die organisationalen Arbeitsplatzplatzmerkmale, wie beispielsweise die Arbeitsinhalte, Aufstiegs- und Weiterbildungsmöglichkeiten, Arbeitszeiten, aber auch die Arbeitsplatzsicherheit und das Betriebsklima, einen hohen Stellenwert ein. Das Ziel der Leistungspolitik besteht darin, die im Rahmen des Arbeitsmarktverhaltens ermittelten Anforderungen von potenziellen Bewerbern möglichst gut zu erfüllen. Die zugrunde liegenden Gestaltungsmöglichkeiten sind allerdings je nach Arbeitsplatz mehr oder weniger begrenzt (KIRCHGEORG und MÜLLER, 2011).

Unter Gehaltspolitik fallen indes gesetzliche, tarifliche und freiwillige betriebliche Sozial- und Nebenleistungen. Hierbei spielt insbesondere die Bestimmung leistungsangemessener Gehaltsniveaus und -entwicklungen eine wichtige Rolle (KIRCHGEORG und MÜLLER, 2011). Ferner sind hierunter die beiden Arbeitsplatzmerkmale Gehaltshöhe und -struktur zu sehen, die als wesentliche entgeltpolitische Instrumente zur Steigerung der Arbeitgeberattraktivität erachtet 
werden (SIMON et al., 1995). Bei der Vergütung wird grundsätzlich zwischen einem fixen Gehaltsbestandteil, der vertraglich festgelegt ist und ohne unmittelbaren Bezug zur erbrachten Leistung gewährt wird, und einem variablen Anteil, dessen Höhe nicht vertraglich festgesetzt, sondern von der Leistung bzw. vom Erfolg abhängt, unterschieden (TUSCHKE, 2011). Zu den Gestaltungsparametern der variablen Vergütung gehören dabei neben der Vergütungshöhe auch die Wahl der Bemessungsgrundlage, die sich ihrerseits aus individuellen Zielvereinbarungen, den Ergebniskennzahlen sowie marktorientierten Kennzahlen zusammensetzt, sowie das mit dem variablen Vergütungsanteil einhergehende Vergütungsrisiko.

Die Kommunikationspolitik wird vor allem innerhalb der Profilierungsfunktion vorausgesetzt und umfasst sämtliche Handlungsschritte, in denen die an die relevante Zielgruppe zu vermittelnden Aussagen samt den dazugehörigen Kommunikationsmaßnahmen festgelegt werden (SIMON et al., 1995). Daneben dient sie der zielgruppenspezifischen Vermittlung der Leistungsund Gehaltspolitik und stellt somit eine entscheidende Voraussetzung zur Steigerung der Arbeitgeberattraktivität dar (KIRCHGEORG und MÜLLER, 2011). Einer Organisation stehen hierzu unterschiedliche Maßnahmen zur Verfügung, die sich zugleich in ihrer Möglichkeit, die von Bewerbern präferierte Spezifität oder Informationsmenge zu präsentieren, deutlich voneinander unterscheiden (vgl. BARBER und ROEHLING, 1993; GATEWOOD et al., 1993; COBER et al., 2004; ROBERSON et al., 2005; ALLEN et al., 2007; COLLINS, 2007).

Eine weitere Strukturierungsebene des Personalmarketings bietet das unten dargestellte Prozessmodell von MOSER und ZEMPEL (2006), das auf einem idealtypischen Rekrutierungsverlauf basiert.

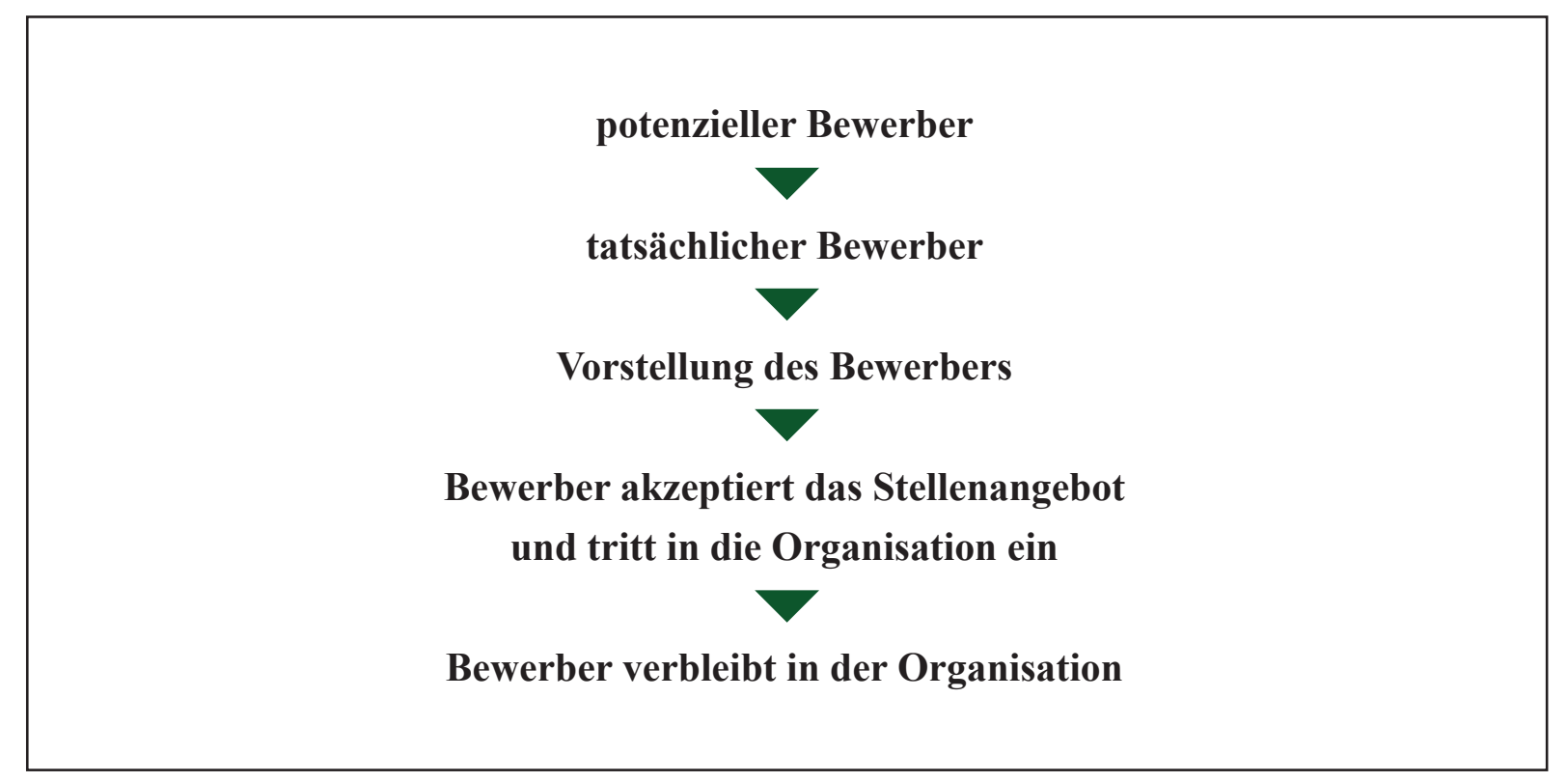

Abb. 2-1 : Personalmarketing als Prozess (MOSER und ZEMPEL, 2006) 
Den Ausgangspunkt bildet hierbei die sog. Attraktion, in der die Ansprache von potenziellen Bewerbern stattfindet. Potenzielle Bewerber können dabei über diverse Rekrutierungskanäle angesprochen und dadurch als tatsächliche Bewerber gewonnen werden, die sich beim Unternehmen vorstellen. Nach der Annahme eines Stellenangebots verbleiben sie typischerweise zunächst im Unternehmen. Dabei wird angenommen, dass sämtliche Phasen des Personalmarketings auf einem wechselseitigen Auswahlprozess zwischen dem Bewerber einerseits und dem Unternehmen andererseits beruhen. Eine prozessuale Differenzierung des Personalmarketings setzt zugleich voraus, dass sich Bewerber an jedem Entscheidungspunkt entschließen könnten, ihr Interesse an der zu besetzenden Stelle aus unterschiedlichen Gründen aufzugeben. Demzufolge besteht die zentrale Aufgabe des Personalmarketings darin, die in den einzelnen Auswahlphasen stattfindenden Entscheidungen der Bewerber zu beeinflussen.

\subsection{Instrumente der externen Bewerberansprache}

Die folgenden Darstellungen konzentrieren sich auf die Maßnahmen zur externen Bewerberansprache, da sie die relevanten Kommunikationsmaßnahmen im Personalmarketing umfassen und somit die Basis einer erfolgreichen Rekrutierung darstellen (SIMON et al., 1995; TURBAN, 2001; COLLINS, 2007). Die konkrete Ausgestaltung eines Rekrutierungsprozesses kann anhand zahlreicher Instrumente und Medien erfolgen, zu deren Strukturierung an dieser Stelle das Elaborations-Wahrscheinlichkeits-Modell (ELM) von PETTY und CACIOPPO (1986) herangezogen wird. Die Theorie wurde im Kontext der sozialpsychologischen Forschung zur Einstellungsänderung entwickelt, lässt sich aber gezielt auf das Feld des Personalmarketings übertragen (CABLE und TURBAN, 2003; ROBERSON et al., 2005). Die zentrale theoretische Annahme des ELM besteht darin, dass Menschen nach zutreffenden Einstellungen streben, die sie anschließend zu richtigen Entscheidungen befähigen sollen. Zugleich verhindert die große, stets auf sie einströmende Informationsmenge eine extensive Verarbeitung sämtlicher Informationen, sodass zwangsläufig eine selektive Informationsverarbeitungsstrategie erforderlich wird. Eine weitere Kernannahme des Modells ist darin zu sehen, dass hier von zwei verschiedenen Informationsverarbeitungsrouten ausgegangen wird, die man als zentral und peripher bezeichnet. Im Rahmen der zentralen Informationsverarbeitung werden alle verfügbaren Informationen zunächst gebündelt und detailliert analysiert, bevor es zu einer Einstellungsänderung kommt. Dieser Prozess wird primär von der Informationsgüte und weniger von der Informationsquelle oder der Präsentationsart beeinflusst. Insgesamt handelt es sich hierbei um einen sehr aufwendigen Informationsverarbeitungsprozess. Demgegenüber wird innerhalb der peripheren Informationsverarbeitung keine umfassende rationale Bewertung des Einstellungsobjektes vorgenommen. Stattdessen werden zur Einstellungsbildung bzw. -änderung diverse Hinweisreize und Heuristiken, wie beispielsweise die Art der Informationspräsentation oder die Informationsquelle, herangezogen (BURMANN und SCHAEFER, 2005). Dabei postulieren PETTY und CACIOPPO 
(1986) weiterhin, dass die Wahl der Informationsverarbeitungsroute von mehreren Faktoren abhängt, wobei das persönliche Involvement in diesem Kontext einen hohen Stellenwert einnimmt. So konnte in mehreren empirischen Studien ein deutlicher Zusammenhang zwischen dem Involvement und der Art der Informationsverarbeitung nachgewiesen werden (vgl. für einen Überblick: PETTY et al., 1997). Demzufolge wird bei einem hohen Involvement eher die zentrale Informationsverarbeitungsroute gewählt, während niedriges Involvement bevorzugt zur peripheren Informationsverarbeitungsroute führt.

Bei den vielfältigen Methoden der externen Bewerberansprache lassen sich demnach deutliche Unterschiede hinsichtlich der jeweils bevorzugten Informationsverarbeitungsroute erwarten (CABLE und TURBAN, 2003; ROBERSON et al., 2005). Dabei wird zum einen unterschieden, ob die eingesetzten Instrumente eine persönliche oder unpersönliche Kommunikation gegenüber dem Bewerber ermöglichen. Und zum anderen wird gefragt, ob die Kontaktaufnahme zum Bewerber direkt oder indirekt erfolgt (SÜSS, 1996). Eine persönliche Kommunikation ist vor allem dadurch gekennzeichnet, dass sie ohne zwischengeschaltete Medien auskommt. Demzufolge sind Kommunikationsmaßnahmen wie Praktika, Vorträge von Unternehmensvertretern in Lehrveranstaltungen und Unternehmensmessen als persönlich-direkt zu kennzeichnen. Gespräche mit potenziellen Bewerbern, die sich aus der Ansprache von Meinungsführern, z.B. Professoren, ergeben, sind indes der persönlich-indirekten Kommunikationsform zuzuordnen. Im Vergleich zu den unpersönlichen Kommunikationsinstrumenten wird den persönlichen Rekrutierungskanälen eine hohe Wahrnehmungswirkung bei der relevanten Zielgruppe bescheinigt (KIRCHGEORG und MüLLER, 2011). Demgegenüber lässt sich die unpersönliche Kommunikation vor allem durch einen intensiven Medieneinsatz charakterisieren. So greift die unpersönlich-direkte Kommunikation auf Individualmedien zurück, wie sie beispielsweise in den per Mail oder Brief zugestellten ImageBroschüren vorzufinden sind. Allerdings geht mit solchen Kommunikationsmedien auch ein vergleichsweise beschränkter Wirkungskreis einher, da die Broschüren lediglich auf entsprechenden Rekrutierungsveranstaltungen verteilt oder auf expliziten Wunsch potenzieller Bewerber ausgehändigt werden. Davon abzugrenzen sind Stellen- und Imageanzeigen, die als Massenmedien fungieren und daher zur Kategorie der unpersönlich-indirekten Kommunikation zuzuordnen sind. Als vorteilhaft erweist sich bei dieser Kommunikationsform, dass sie aufgrund ihres hohen Verbreitungsgrades einen vergleichsweise großen Personenkreis erreichen können, in dem das Unternehmen bislang noch nicht bekannt war.

Grundsätzlich wird davon ausgegangen, dass der persönliche und direkte Kontakt zwischen Unternehmen und potenziellen Bewerbern im Vergleich zur Massenmedienkommunikation die zentrale Informationsverarbeitung begünstigt (CABLE und TURBAN, 2003). Ursächlich hierfür ist SÜSS (1996) zufolge die mit dem persönlichen Kontakt einhergehende höhere Glaubwürdigkeit, die stärkeren sozialen Kontrollmöglichkeiten sowie die größere Flexibilität beim 
gegenseitigen Informationsaustausch. Des Weiteren wird angenommen, dass die Merkmale der in den Kommunikationsprozess einbezogenen Personen die Informationsverarbeitungsart beeinflussen (LARSEN und PHILIIPS, 2002). So zeigen entsprechende Studien, dass das Interviewerverhalten, das beispielsweise im Rahmen einer Firmenmesse gezeigt wird, die Wahrnehmung der Merkmale der Organisation bzw. der Tätigkeit beeinflusst (TURBAN et al., 1998). In der folgenden Übersicht werden einige Möglichkeiten zur Bewerberansprache der Zielgruppe Studierende und Hochschulabsolventen aufgezeigt.

\begin{tabular}{|c|c|}
\hline Persönlich-direkte Bewerberansprache & Persönlich-indirekte Bewerberansprache \\
\hline $\begin{array}{l}\text { - Praktika, Werkstudententätigkeiten } \\
\text { - Projekt- und Abschlussarbeiten } \\
\text { - Unternehmenspräsentationen an Hochschulen } \\
\text { - Unternehmensexkursionen } \\
\text { - Vorträge von Unternehmensvertretern } \\
\text { in Lehrveranstaltungen } \\
\text { - Job- und Fachmessen außerhalb der Hochschule }\end{array}$ & $\begin{array}{l}\text { - Kontakte zu Professoren/Lehrenden relevanter } \\
\text { Fachrichtungen }\end{array}$ \\
\hline Unpersönlich-direkte Bewerberansprache & Unpersönlich-indirekte Bewerberansprache \\
\hline $\begin{array}{l}\text { - Imagewerbung in Unternehmensbroschüren } \\
\text { - Werbung auf Social Media Plattformen } \\
\text { (z.B. Facebook, Xing) }\end{array}$ & $\begin{array}{l}\text { - Stellenanzeigen in Zeitschriften und Zeitungen } \\
\text { - Stellenanzeigen im Internet (z.B. Online-Jobbörsen) } \\
\text { - Hochschuleigene Datenbanken }\end{array}$ \\
\hline
\end{tabular}

Tab. 2-1: Ausgewählte Instrumente des Personalmarketings

(Eigene Darstellung, 2010, in Anlehnung an SÜSS, 1996)

Zur Bewertung der unterschiedlichen Kommunikationsmaßnahmen ist grundsätzlich eine Reihe von Evaluationskriterien denkbar (MOSER und ZEMPEL, 2006). So könnten diese hinsichtlich ihrer Bekanntheit, ihres Informationsgehalts, ihrer Aktualität und ihrer Kosten, ihrer Erhältlichkeit und Zugänglichkeit, ihrer Glaubwürdigkeit und Nützlichkeit sowie ihres Potenzials, das Interesse von potenziellen Bewerbern zu wecken, beurteilt werden. Allerdings erweist sich eine solches Vorhaben aufgrund mehrerer methodischer Aspekte als problematisch. So werden neue Mitarbeiter häufig über mehrere Wege gleichzeitig erreicht (MARCUS, 2011). Ferner erlauben die oben dargestellten Kommunikationsmaßnahmen durchaus Gestaltungsspielräume, was beispielsweise die konkrete Vermittlung von Informationen über den Arbeitsplatz angeht (ROBERSON et al., 2005). Vor diesem Hintergrund ist die Generalisierung der Befragungsergebnisse aus evaluativen Studien nur sehr bedingt möglich. 


\subsection{Stellenwert des Personalmarketings innerhalb der Ernährungsindustrie}

Um den Stellenwert des Personalmarketings in der Ernährungsindustrie besser einschätzen zu können, wird zunächst die zugrunde liegende Branchenstruktur näher beschrieben. Dabei wird im ersten Schritt die Relation zwischen den einzelnen Branchenzweige anhand der beiden Kriterien Unternehmens- und Mitarbeiteranzahl veranschaulicht. Anschließend werden die zur Branche gehörenden Unternehmen entsprechend ihrer Unternehmensgröße klassifiziert.

\begin{tabular}{|c|l|l|l|}
\hline \multicolumn{2}{|c|}{ Branchengruppen } & $1198(23,5 \%)$ & $112610(17,8 \%)$ \\
\hline 10.1 & Schlachten und Fleischverarbeitung & $58(1,1 \%)$ & $7526(1,2 \%)$ \\
\hline 10.2 & Fischverarbeitung & $210(4,1 \%)$ & $27170(4,3 \%)$ \\
\hline 10.3 & Obst- und Gemüseverarbeitung & $24(0,5 \%)$ & $6307(1,0 \%)$ \\
\hline 10.4 & Herstellung von pflanzlichen und tierischen Ölen und Fetten $\%$ & $138(2,7 \%)$ & $36189(5,7 \%)$ \\
\hline 10.6 & $\begin{array}{l}\text { Mahl- und Schälmühlen, Herstellung von Stärke } \\
\text { und Stärkeerzeugnissen }\end{array}$ & $83(1,6 \%)$ & $11556(1,8 \%)$ \\
\hline 10.7 & Herstellung von Back- und Teigwaren & $2390(47,0 \%)$ & $268885(42,4 \%)$ \\
\hline 10.8 & Herstellung von sonstigen Nahrungsmitteln & $382(7,5 \%)$ & $86674(13,7 \%)$ \\
\hline 10.9 & Herstellung von Futtermitteln & $118(2,3 \%)$ & $13064(2,1 \%)$ \\
\hline 11.0 & Getränkeherstellung & $\mathbf{5 0 8 8}(\mathbf{1 0 0} \%)$ & $\mathbf{6 3 4 1 0 9}(\mathbf{1 0 0} \%)$ \\
\hline Gesamt & & & \\
\hline
\end{tabular}

Tab. 2-2: Anzahl von Unternehmen und Mitarbeitern in der Ernährungsindustrie mit 20 und mehr Beschäftigten im Jahr 2009 in Deutschland (Eigene Darstellung, 2010, in Anlehnung an STATISTISCHES BUNDESAMT, 2010)

Die deutsche Ernährungsindustrie bestand demzufolge im Jahr 2009 aus insgesamt 5088 Unternehmen, in denen ca. 634 Tsd. Mitarbeiter beschäftigt waren. Die einzelnen Branchenzweige waren hierbei unterschiedlich stark vertreten. Die Branchengruppe Herstellung von Back- und Teigwaren setzt sich sowohl hinsichtlich der Unternehmens- als auch der Mitarbeiteranzahl deutlich von den übrigen Branchenzweigen ab. An zweiter Stelle befindet sich der Branchenzweig Schlachten und Fleischverarbeitung, gefolgt von der Herstellung von sonstigen Nahrungsmitteln sowie der Getränkeherstellung. 
Die Analyse der Unternehmensgrößenstruktur in der Ernährungswirtschaft erfordert eine fest definierte Unternehmensgrößenabgrenzung. Hierzu existiert allerdings bisher keine allgemeingültige Definition, vielmehr verfügen unterschiedliche nationale und internationale Institutionen über eigene Klassifizierungsansätze. Einen in der Wissenschaft und Praxis weit verbreiteten Ansatz zur Unternehmensgrößenabgrenzung stellt die Definition des Instituts für Mittelstandsforschung Bonn (IfM) dar. Die Unternehmensgrößenabgrenzung erfolgt hierbei anhand der beiden quantitativen Kriterien Beschäftigtenzahl und Jahresumsatz.

\begin{tabular}{|c|c|c|}
\hline Unternehmensgröße & Beschäftigtenzahl & Umsatz Euro/Jahr \\
\hline klein & bis 9 & bis unter 1 Mio. \\
\hline mittel & 10 bis 499 & 1 bis 50 Mio. \\
\hline groß & 500 und mehr & 50 Mio. und mehr \\
\hline
\end{tabular}

Tab. 2-3: Unternehmensgrößenabgrenzung des Instituts für Mittelstandsforschung

(Eigene Darstellung, 2010, in Anlehnung an INSTITUT FÜR MITTELSTANDFORSCHUNG, 2002)

Davon zu unterscheiden ist die Klassifikation der Europäischen Kommission, in der die Einteilung der Unternehmen anhand des zusätzlichen Kriteriums Jahresbilanzsumme erfolgt.

\begin{tabular}{|c|c|c|c|}
\hline Unternehmensgröße & Beschäftigtenzahl & Umsatz EUR/Jahr & Bilanzsumme EUR/Jahr \\
\hline kleinst & weniger als 10 & $\max$. 10 Mio. & max. 10 Mio. \\
\hline klein & weniger als 50 & $\max$. 50 Mio. & max. 43 Mio. \\
\hline
\end{tabular}

Tab. 2-4: Unternehmensgrößenabgrenzung der Europäischen Kommission

(Eigene Darstellung, 2010, in Anlehnung an EUROPÄISCHE KOMMISSION, 2005)

Aufgrund ihres hohen Verbreitungsgrades in Wissenschaft und Praxis wird in den folgenden Ausführungen auf die Klassifikation des Instituts für Mittelstandsforschung Bonn zurückgegriffen. Der Mittelstand umfasst demnach alle Unternehmen, in denen nicht mehr als 499 Mitarbeiter beschäftigt werden. In der nachfolgenden Übersicht wird die Unternehmensanzahl in den unterschiedlichen Unternehmensgrößenklassen der Ernährungsindustrie beispielhaft für das Jahr 2009 dargestellt. 


\begin{tabular}{|c|c|}
\hline $\begin{array}{c}\text { Größenklassen der Unternehmen } \\
\text { (nach Anzahl der Beschäftigten) }\end{array}$ & Anzahl der Unternehmen für das Jahr 2009 \\
\hline $20-49$ & 2.288 \\
\hline $50-99$ & 1.303 \\
\hline $100-249$ & 976 \\
\hline $250-499$ & 333 \\
\hline 500 und mehr & 18 \\
\hline gesamt & $\mathbf{5 . 0 8 8}$ \\
\hline
\end{tabular}

Tab. 2-5: Struktur der produzierenden Ernährungsindustrie für Unternehmen mit 20 und mehr tätigen Personen (Eigene Darstellung, 2010, in Anlehnung an STATISTISCHES BUNDESAMT, 2010)

Der oben angeführten Definition von mittelständischen Unternehmen folgend wird aus der obigen Tabelle ersichtlich, dass die meisten Unternehmen der Ernährungsindustrie gemäß dem Kriterium Beschäftigtenzahl als mittelständisch zu klassifizieren sind. Dabei lässt sich für mittelständische Unternehmen generell konstatieren, dass sie sich zunehmend erschwerten Akquisitionsbedingungen ausgesetzt sehen, da sie einerseits untereinander, aber auch mit Großunternehmen um besonders qualifizierte Mitarbeiter konkurrieren (SENG und STARYSTACH, 2010). Die erschwerte Wettbewerbssituation liegt vor allem darin begründet, dass mittelständische Unternehmen zumeist nur in ihrer Region bekannt sind und über eine vergleichsweise ungünstige Positionierung auf dem Arbeitsmarkt verfügen, wodurch wiederum ihre Profilierungsfunktion spürbar beeinträchtigt wird (SCHMIDTKE, 2001). Gleichzeitig werden mittelfristig erhebliche Verknappungserscheinungen bei der generellen Verfügbarkeit von Mitarbeitern prognostiziert, die sich auf den demographischen Wandel und die damit einhergehende Minderung des Erwerbspersonenpotenzials zurückführen lassen (KAY et al., 2010).

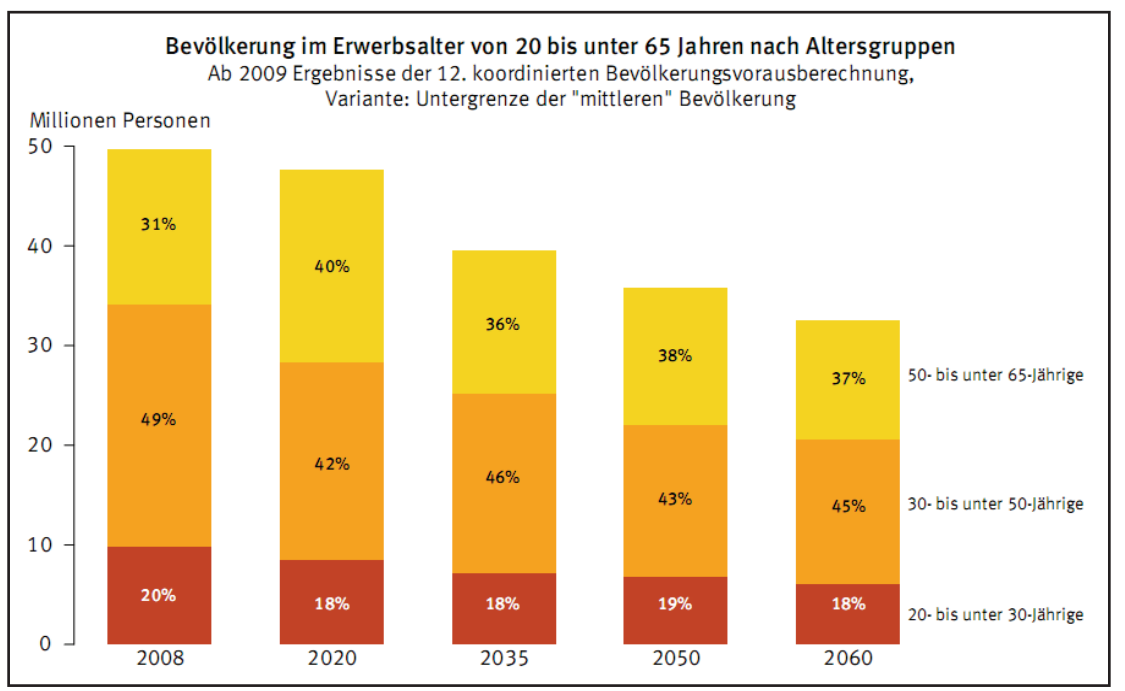

Abb. 2-2: Entwicklungsprognose des Erwerbspotenzials in Deutschland (STATISTISCHES BUNDESAMT, 2009) 
Außer dem demographisch verursachten Mangel an Fach- und Führungskräften nimmt der Stellenwert des Personalmarketings auch aufgrund weiterführender Entwicklungen insgesamt zu (BÖTTGER, 2012). Dazu zählen neben dem Wertewandel auch der zunehmend international ausgetragene Wettbewerb um geeignete Nachwuchskräfte sowie die qualitativ gestiegenen Anforderungen an potenzielle und tatsächliche Mitarbeiter. Inzwischen liegen erste branchenübergreifende Belege dafür vor, dass mittelständische Unternehmen die Rekrutierung von hochqualifizierten Nachwuchskräften bereits zum jetzigen Zeitpunkt für besonders wichtig für ihren Unternehmenserfolg erachten und auch langfristig die Bedeutung von Mitarbeitergewinnung realisiert haben (SENG und STARYSTACH, 2010). Gleichzeitig lassen sich deutliche Abweichungen in den Personalmarketingaktivitäten zwischen den klein- und mittelständischen und den Großunternehmen beobachten, wobei Großunternehmen naturgemäß mehr finanzielle und personelle Ressourcen zur Deckung ihres qualitativen und quantitativen Personalbedarfs aufwenden. Ebenso variieren die angestrebten Zielgruppen mit der Unternehmensgröße. So konnten SENG und STARYSTACH (2010) in ihrer Studie zeigen, dass Großunternehmen Schüler, Studierende und Absolventen gleichermaßen als relevante Zielgruppe betrachten, während mittelständische Unternehmen sich verhältnismäßig stark an Studierende und Hochschulabsolventen richten.

Betrachtet man explizit die Ernährungsindustrie, so zeigt sich, dass die Verfügbarkeit von geeigneten Mitarbeitern auch in dieser Branche von den Unternehmen zunehmend als Problem erkannt wird (THEUVSEN et al. 2009). Aus der praxisorientierten Studie der AFC Unternehmensberatung geht zugleich hervor, dass insbesondere die Unternehmensbereiche Produktion, die technischen Abteilungen sowie die Forschungs- und Entwicklungsabteilung von dieser Entwicklung betroffen sind (ELLES et al., 2012). Aus diesen Ergebnissen lässt sich somit ableiten, dass auch in der Ernährungsindustrie mittelfristig ein Wandel vom Arbeitgeber- zum Arbeitnehmermarkt stattfinden wird. Hierdurch steigt auch gleichzeitig die Bedeutung von professionellen Personalmarketing-Prozessen innerhalb des Personalmanagements (KIRCHGEORG und MÜLlER, 2011). Eine entsprechende Professionalisierung macht es erforderlich, eine branchenspezifische Situationsanalyse durchzuführen, um die darauf basierenden Strategien und Instrumente präzise auf die relevanten Zielgruppensegmente für die Personalakquisition auszurichten.

\subsection{Empirische Perspektive des Personalmarketings}

\subsubsection{Wissenschaftliche Studien zum Personalmarketing}

Im Fokus zahlreicher empirischer Studien des Personalmarketings steht die Frage, welche Bewerberpräferenzen bei der Arbeitgeberwahl ausschlaggebend sind. Bei einer genaueren Betrachtung der hierzu vorliegenden Literatur wird allerdings deutlich, dass eine Vielzahl der entsprechenden Studien aufgrund ihrer kommerziellen Ausrichtung und deshalb fehlenden Ob- 
jektivität wissenschaftlichen Kriterien nicht gerecht wird. Überdies zielen diese Ansätze weniger auf die Sicherstellung theoretischer Erkenntnisse als vielmehr auf die Entwicklung und Bereitstellung unmittelbar umsetzbarer Gestaltungsempfehlungen für Unternehmen und andere Institutionen ab. In Abgrenzung dazu stützt sich der folgende Literaturüberblick ausschließlich auf wissenschaftliche Beiträge, um den aktuellen Forschungsstand zur Bedeutung von Arbeitsplatzmerkmalen möglichst fundiert zu veranschaulichen.

WILTINGER (1997) analysiert in seiner Studie die Bewerberpräferenzen von kaufmännischen Nachwuchsführungskräften. Anhand von insgesamt acht Arbeitsplatzmerkmalen, wie beispielsweise Gehalt, Sozialleistungen und Weiterbildungsmöglichkeiten, untersucht der Autor mithilfe der sog. Conjoint-Analyse (CA), inwieweit sich diese auf die Präferenz von Stellenangeboten auswirken. Bei den Befragten handelte es sich in dieser Studie ausschließlich um Studierende wirtschaftswissenschaftlicher Studiengänge. Dabei zeigte sich, dass dem Einkommen anders als bei der Verwendung direkter Befragungsmethoden ein hoher Stellenwert beigemessen wird, wobei Aufstiegs- und Karrierechancen in dieser Studie als noch bedeutsamer eingeschätzt wurden. WiLTINGER (1997) leitet aus den Ergebnissen insgesamt ab, dass Unternehmen ihr nicht optimal ausgeprägtes Arbeitgeberimage durch eine flexible Gehaltspolitik zumindest teilweise kompensieren können.

In einer ähnlichen Weise untersuchen SCHWOERER und ROSEN (1989) in ihrer experimentell angelegten Studie das Verhältnis der Gehaltshöhe zu anderen Arbeitsplatzmerkmalen. Die Ausgangsfrage besteht hierbei darin, wie unterschiedliche Kombinationen der beiden Merkmale Gehalt und Arbeitsplatzsicherheit auf die wahrgenommene Arbeitgeberattraktivität und damit einhergehende Bewerbungsbereitschaft wirken. Es zeigte sich, dass eine bessere Bezahlung und ein sicherer Arbeitsplatz zu einer besseren Beurteilung des Unternehmens führen, während in der Bedingung mit einer willkürlichen Kündbarkeit und einem durchschnittlichen Gehalt die geringste Bewerbungsbereitschaft hervorgeht. Darüber hinaus ist die Bewerbungsbereitschaft in der Bedingung mit einem überdurchschnittlichen Gehalt und einer willkürlichen Kündbarkeit größer als bei einer sicheren Stellung mit durchschnittlichem Gehalt. Auch aus den Ergebnissen der Studie von SAKS et al. (1996) geht hervor, dass sich ein überdurchschnittliches Gehalt positiv auf die Beurteilung anderer Arbeitsplatzmerkmale und gleichzeitig auf die globale Arbeitgeberattraktivität auswirkt. Der Zusammenhang zwischen dem Gehalt und der Arbeitgeberattraktivität bzw. Bewerbungsbereitschaft konnte überdies in weiteren empirischen Studien mit indirekter Befragungstechnik bestätigt werden (z.B. CABLE und GRAHAM, 2000; AIMAN-SMITH et al., 2001; ANDRATSCHKE et al., 2009; CASPER und BUFFARDI, 2004).

Die Ergebnisse der oben dargestellten Studien sind deshalb von so großem Interesse, da die empirische Forschung sich intensiv mit dem Arbeitsplatzmerkmal Gehalt befasst hat und hierbei zu abweichenden, teilweise widersprüchlichen Ergebnissen gelangt (MOSER und ZEMPEL, 2006). So existiert eine Reihe von Untersuchungen, in denen das Gehalt insgesamt eine unter- 
geordnete Rolle für die Arbeitgeberwahl einnimmt (SCHWAAB, 1991; HONEYCUTT und ROSEN, 1997; LEWANDOWSKI und LIEBIG, 2004; BÖTTGER, 2012). Diese Ergebnisse stehen zugleich im Einklang mit den theoretischen Überlegungen und empirischen Befunden zum Wertewandel (ROSENSTIEL und NERDINGER, 2000). Darunter wird der seit den 60er Jahren in repräsentativen Stichproben beobachtete Wertewandel verstanden, in dem postmaterialistische Werte im Vergleich zu materialistischen als zunehmend wichtiger erachtet werden (INGLEHART, 1990). Werte werden in diesem Kontext als eine Konzeption des Wünschenswerten definiert, die dem Handeln Orientierung geben und dabei die Auswahl der verfügbaren Handlungen und Ziele beeinflussen (KLUCKHOHN, 1951, zit. in ROSENSTIEL und NERDINGER, 2000). Materialistische Werte umfassen INGLEHART (1990) zufolge die Bereiche Sicherheit und Versorgung, während postmaterialistische Werte auf die Bereiche Selbstverwirklichung sowie Solidarität ausgerichtet sind. Materialismus und Postmaterialismus stellen innerhalb seiner Konzeption zwei Extrempunkte auf einer Dimension dar, sodass der sich vollziehende Wertewandel als eine Verschiebung zum Pol des Postmaterialismus beschrieben werden kann. Innerhalb der Arbeitgeberwahl schlägt sich der Wertewandel insofern nieder, als dass die Bewertung bzw. Präferenz bestimmter Arbeitsplatzmerkmale auf die zugrunde liegenden beruflichen Werthaltungen von potenziellen Bewerbern zurückzuführen sind (JUDGE und BRETZ, 1992; LANG VON-WINS et al., 1995). Diese theoretische Annahme konnte in einer Reihe von empirischen Studien, die sowohl auf Basis qualitativer als auch quantitativer Methoden im Quer- und Längsschnittdesign durchgeführt wurden, teilweise bestätigt werden (ROSENSTIEL und NERDINGER, 2000). So zeigten die Ergebnisse der Studie von LANG VON-WINS et al., 1995, dass eine hohe Gewichtung materieller Werthaltungen zur einer höheren Bewertung von belohnungsbezogenen Arbeitsplatzmerkmalen, wie beispielsweise der Bezahlung oder der vom Unternehmen gewährten Sozialleistungen, führt. Demgegenüber resultieren postmaterialistische Werthaltungen in einer deutlichen Präferenz für arbeitsinhaltliche und organisationale Aspekte, die ihrerseits beispielsweise in guten Weiterbildungsmöglichkeiten oder der sog. Corporate Social Responsibility (CSR) zu finden sind (LIS, 2010). Dementsprechend kommt die Zunahme postmaterialistischer Werthaltungen darin zum Ausdruck, dass im Rahmen der Arbeitgeberwahl weniger als früher die materialistischen Inhalte des beruflichen Erfolgs angestrebt werden, sondern vermehrt soziale oder umweltorientierte Facetten eines Unternehmens als bedeutsam erachtet werden (TURBAN und GREENING, 1996; BAUER und AIMAN-SMITH, 1998; BACKHAUS et al., 2002).

Ein alternatives Erklärungsmuster für die Bedeutung des Arbeitsplatzmerkmals Gehalt besteht in der Annahme, dass seine Bedeutsamkeitseinschätzung von der zugrunde liegenden Arbeitsmarktlage abhängt (LANG VON-WINS et al., 1995; CABLE und JUDGE, 1996). Darüber hinaus konnten RYNES et al. (1983) in ihrer experimentellen Studie zeigen, dass das Gehalt nicht als entscheidungsrelevant beurteilt wird, solange es individuellen Minimalstandards genügt. Diesem Befund liegen die nicht-kompensatorischen Entscheidungsregeln zugrunde, denen zufolge eine Option nicht gewählt wird, wenn sie nicht bestimmte Schwellenwerte auf einem oder mehreren Attributen überschreitet, unabhängig davon, wie gut sie auf anderen Attributen 
abschneidet (JUNGERMANN et al., 2010). In ihrer Untersuchung fanden RYNES et al. (1983) Belege für beide Erklärungsmuster: Sowohl die Annahme über die Wirkung von Marktparametern als auch die Wirkung nicht-kompensatorischer Entscheidungsregeln konnten gestützt werden. Allerdings ist bei den zugrunde liegenden Studien die Stichprobenabhängigkeit der Ergebnisse zu beachten. So hebt NERDINGER (1994) in diesem Zusammenhang hervor, dass die meisten Untersuchungen, in denen minimale Standards bezüglich des Gehalts bestätigt wurden, an erwerbslosen Personen durchgeführt wurden, deren Entscheidungsverhalten bei der Arbeitgeberwahl sich deutlich von demjenigen der Akademiker unterscheidet.

Eine weitere Erklärungsoption ist im sog. Bandbreiteneffekt zu sehen, dem zufolge die Wichtigkeit eines Attributs von seiner Bandbreite, d.h. der Differenz zwischen seiner geringsten und höchsten Ausprägung abhängt (SATTLER, 2006). So stellten RYNES et al. (1983) fest, dass die Bedeutung der Eigenschaft Gehalt zunimmt, wenn große Unterschiede in der Gehaltshöhe zwischen den einzelnen Eigenschaftsausprägungen bestehen. Hierbei ist somit zwischen der relativen und absoluten Wichtigkeit des Arbeitsplatzmerkmals Gehalt zu differenzieren: Es kann zwar über eine große absolute Bedeutsamkeit verfügen, seine relative Wichtigkeit kann dabei allerdings gering ausfallen, wenn sich zwei oder mehrere Stellenangebote hinsichtlich des Gehalts kaum unterscheiden (JUNGERMANN et al., 2010).

Die Widersprüchlichkeit der vorliegenden Forschungsergebnisse hinsichtlich des Arbeitsplatzmerkmals Gehalt lässt sich auf mehrere Faktoren zurückführen. Zum einen weist die Operationalisierung des Gehalts keine inhaltliche Konsistenz auf. Während in manchen Studien mehrere, in ihrer Höhe unterschiedliche Gehaltskategorien vorgegeben werden, greifen andere Autoren bei der Itemkonzeption auf Formulierungen wie „hohes Einstiegsgehalt“, „,schnelle Gehaltsprogression“, ,,attraktive Gesamtvergütung“ oder schlicht „Kompensation/Gehalt“ zurück (vgl. z.B. WiLTINGER, 1997; LEWANDOWSKI und LIEBIG, 2004; KNOX und FREEMAN, 2006; ANDRATSCHKE et al., 2009; BÖTTGER, 2012).

Fernerhin geht die Operationalisierung von Arbeitsplatzmerkmalen mit erheblichen methodischen Differenzen einher, die sich ihrerseits auf die Ergebnisse auswirken. Die meisten der oben dargestellten Studien werden mittels direkter Befragungsmethoden, wie der Ratingskala oder der Rangreihenbildung, umgesetzt. Darin geben Befragte auf einer vorgegebenen Liste an, welche Arbeitsplatzmerkmale sie bei der Beurteilung eines Arbeitgebers als besonders relevant erachten. Währenddessen liegen indirekten Befragungsmethoden nicht einzelne Arbeitsplatzmerkmale, sondern unterschiedliche Arbeitsplatzbeschreibungen zugrunde, die aus einer systematisch variierten Kombination von Arbeitsplatzmerkmalen bestehen (AIMAN-SMITH et al., 2002). Auf dieser Grundlage bewerten die befragten Personen die Gesamtattraktivität von potenziellen Arbeitgebern oder sie entscheiden, ob sie sich bewerben und das Stellenangebot annehmen würden. Die Relevanz einzelner Arbeitsplatzmerkmale wird dementsprechend über ihre Gewichtung bei der Entscheidungsvorhersage erschlossen (KARREN und BARRINGER, 
2002). Die Differenzen zwischen der direkten und indirekten Befragungsmethode werden beispielsweise anhand der Untersuchung von WILTINGER (1997) deutlich, in der die Aufstiegs- und Karrierechancen sowie das Gehalt im Rahmen seiner Conjoint-Analyse eine größere, Weiterbildung, Führungsstil und Sozialleistungen hingegen eine niedrigere Gewichtung als bei direkten Befragungen erhielten. Diese methodenbedingte Abweichung in der Bedeutsamkeitseinschätzung von Arbeitsplatzmerkmalen, die auch in weiteren empirischen Studien zu beobachten ist, führt der Autor auf den Effekt der sozialen Erwünschtheit zurück. Ein umittelbarer empirischer Beleg für den beachtlichen Effekt der sozialen Erwünschtheit bei der Bewertung von Arbeitsplatzmerkmalen geht aus der Untersuchung von ARNOLD und FELDMAN (1981) hervor, in der Befragte mit starker Tendenz zur sozialen Erwünschtheit größere Differenzen zwischen direkten und indirekten Methoden zeigten. Auch TEUFER (1999) stellt in diesem Kontext fest, dass einzelne Arbeitsplatzmerkmale deutlichen Effekten der sozialen Erwünschtheit unterliegen, was sich besonders in der Frage des Gehalts widerspiegelt. Vor diesem Hintergrund heben MOSER und ZEMPEL (2006) die Vorteile von indirekten Befragungsmethoden hervor, die insbesondere darin zu sehen sind, dass die so ermittelten Bewerberpräferenzen weniger dem Effekt sozialer Erwünschtheit unterliegen und auch die Art, wie Entscheidungen getroffen werden, dem Vorgehen in der Realität vergleichsweise nahe kommt. Eine ausführliche und weiterführende Darstellung der indirekten Befragungsmethoden, wie sie auch in dieser Arbeit verwendet werden, befindet sich im Abschnitt 5.1.

Ein weiteres methodisches Problem lässt sich im häufig verwendeten Ist-Soll-Vergleich zwischen den in einem Unternehmen wahrgenommenen und den erwarteten bzw. angestrebten Arbeitsplatzmerkmalen lokalisieren. Ein solches Vorgehen umfasst eine zweistufige Messung, wobei im ersten Schritt die Bewerbererwartungen im Sinne eines Soll-Profils erhoben werden, um diesen anschließend die konkrete Wahrnehmung eines bestimmten Unternehmens gegenüberzustellen (vgl. z.B. SCHWAAB, 1991; TURBAN, 2001; LEWANDOWSKI und LIEBIG, 2004; LiEVENS et al., 2007). Aus dem Vergleich beider Profile leiten die Autoren Schwachstellen in der Positionierung des Unternehmens als Arbeitgeber ab, die sich durch gezielte Maßnahmen des Personalmarketings beseitigen lassen sollten. Obwohl solche Imagemessungen zur Entwicklung eines eigenständigen Unternehmensprofils empfohlen werden, impliziert dieses Vorgehen aus wissenschaftlicher Sicht mehrere Annahmen, die kritisch zu betrachten sind. Zunächst wird hierbei davon ausgegangen, dass Bewerber über ein genaues Bild ihres idealen Arbeitgebers verfügen. Überdies wird angenommen, dass derartige Idealbilder die Entscheidungen im gesamten Personalmarketingprozess steuern. BOSWELL et al. (2003) konnten in einer qualitativen Längsschnittstudie an College-Absolventen hingegen zeigen, dass eine beträchtliche Anzahl ihrer Befragten die Vorstellungen von einem idealen Arbeitgeber im Laufe des Arbeitsgeberwahlprozesses änderte und ihre Entscheidung schließlich von Arbeitsplatzmerkmalen bestimmt wurde, die zu Beginn dieses Prozesses als nicht bzw. wenig bedeutsam eingestuft wurden. Die Ergebnisse dieser Studie widersprechen folglich der statischen Perspektive des oben dargestellten Ansatzes. 
Darüber hinaus kritisieren MOSER und ZEMPEL (2006), dass die Auswahl der als relevant erachteten Arbeitsplatzmerkmale bzw. Anforderungskriterien häufig ohne eine theoretische Grundlage vorgenommen wird. Stattdessen werden den Befragten möglichst viele unterschiedliche Kriterien zur Bewertung von Arbeitgebern vorgegeben, die im zweiten Schritt mittels faktorenanalytischer Auswertungen zu mehreren Dimensionen verdichtet werden (z.B. LEMMINK et al., 2003; CARLESS und IMBER, 2007; BECKER et al., 2011).

Demgegenüber geht TEUFER (1999) in seiner Studie a priori von mehreren Dimensionsebenen aus, wobei er das allgemeine Arbeitgeberimage hierarchisch in Unternehmens-, Standort- und Branchenimage einerseits sowie in personalpolitische Parameter und den sog. feel good-Faktor andererseits unterteilt. Die Ausgangsfrage besteht auch bei dieser Studie darin, welche Arbeitsplatzmerkmale Studierende bzw. Absolventen bei ihrer Arbeitgeberwahl als bedeutsam einschätzen. Die Operationalisierung der Arbeitsplatzmerkmale erfolgt auf der Grundlage von indirekten Paarvergleichen, sodass die Effekte der sozialen Erwünschtheit innerhalb dieser Untersuchung als gering zu bewerten sind. Aus den Ergebnissen geht hervor, dass die befragten Personen die personalpolitischen Parameter und den feel good-Faktor gefolgt vom Unternehmensimage als wesentliche Dimensionen der Arbeitgeberwahl bewerten, während das Branchen- und Standortimage eine geringe Bedeutsamkeit erfahren. Bemerkenswert ist weiterhin, dass die Arbeitsplatzmerkmale Gehalt und Karrierechancen trotz indirekter Befragungsmethoden als weniger bedeutsam eingestuft wurden als in der Studie von WILTINGER (1997). Kritisch anmerken lässt sich an dieser Studie allerdings, dass die Konstruktvalidität des als entscheidungsrelevant eingestuften feel good-Faktors als niedrig zu betrachten ist. So wird der als Bestandteil des feel good-Faktors fungierende Kollegen-Fit anhand des Ablaufs des Einstellungsinterviews und sowie der Glaubwürdigkeit des Interviewers operationalisiert, was inhaltlich jedoch nicht der theoretischen Konzeption dieses Konstruktes entspricht (KRISTOF, 1996). Vielmehr stellen das Interviewerverhalten und die inhaltlichen Aspekte des Einstellungsverfahrens, wie sie beispielsweise innerhalb des Konzeptes der realistischen Tätigkeitsvorschau zum Tragen kommen, eigenständige Konstrukte dar (LARSEN und PHILIIPS, 2002; CHAPMAN und WEBSTER, 2006). Im Einklang mit dem erhöhten Bedarf nach besonders qualifizierten Nachwuchskräften führt TEUFER (1999) in seiner Studie eine Segmentierung der befragten Personen in sog. High Potentials und übrige Studierende durch. Ein zentrales Ergebnis stellt hierbei der Befund dar, dass die Rangfolge der Arbeitsplatzmerkmale sowohl für High Potentials als auch für die übrigen Studierenden identisch ist. Demgegenüber zeigen sich bedeutsame Unterschiede in der Bewertung einzelner Dimensionen, wobei die High Potentials das Unternehmensimage und den feel good-Faktor höher, die personalpolitischen Parameter hingegen niedriger als die übrigen Studierenden bewerten.

Des Weiteren werden vereinzelt auch persönlichkeitsspezifische Merkmale erhoben und als Segmentierungskriterien in die Analysen einbezogen. Die zugrunde liegenden Operationalisierungen erfolgen häufig allerdings nicht anhand von theoretischen Konstrukten und gestalten 
sich zudem sehr heterogen. So werden unter diesem Oberbegriff persönliche Wertvorstellungen, berufliche Ziele, Persönlichkeitseigenschaften und motivationale Komponenten potenzieller Bewerber erfasst. Infolgedessen ergibt sich insgesamt ein sehr heterogenes Ergebnisbild, das aufgrund der teils stark abweichenden Operationalisierungsstrategien kaum Vergleichsmöglichkeiten zulässt.

In den marketingwissenschaftlichen Studien werden zur Identifikation verschiedener Segmente auf Basis von persönlichkeitsspezifischen Merkmalen überwiegend Clusteranalysen durchgeführt. So zieht FRANKE (2000) in seiner empirischen Untersuchung motivationale Merkmale von Studierenden und Absolventen wirtschaftswissenschaftlicher Studiengänge zur Ableitung von Clustern heran und gelangt auf diesem Wege zu drei in sich homogenen Motivationstypen, die sich hinsichtlich der Bewertung von Arbeitsplatzmerkmalen und der bevorzugten Arbeitgeber grundsätzlich voneinander unterscheiden. Das Konstrukt Motivation wurde in dieser Untersuchung mittels der Komponenten Risikobereitschaft, Sinnorientierung, Leistungs- sowie Teamorientierung erfasst. Die aus der Clusteranalyse resultierenden Typen beschreibt der Autor als Manager, Teamplayer und resignierte Verweigerer. GROBE (2003) spezifiziert in ihrer Studie indes auf der Grundlage von Wichtigkeitsurteilen vier Zielgruppensegmente von Befragten, die sie als die Genügsamen, die Eigennützigen, Ausgeglichenen sowie die Anspruchsvollen bezeichnet. Diese Clusterlösung konnte in weiteren empirischen Arbeiten bestätigt werden (KIRCHGEORG und LORBEER, 2002; KIRCHGEORG und GÜNTHER, 2006; BÖTTGER, 2012). Allerdings greifen die oben genannten Studien bei ihren Erhebungen allesamt auf die Stipendiaten des e-fellows.net Netzwerks zurück, sodass die daraus resultierenden Befunde möglicherweise einer systematischen Verzerrung unterliegen und im Sinne der externen Validität nicht ohne Weiteres auf die Grundgesamtheit von High Potentials zu übertragen sind. Ähnlich geht SCHAMBERGER (2006) vor, dessen Clusterbildung auf den beruflichen Werthaltungen von wirtschaftswissenschaftlichen Studierenden und Absolventen, die ebenfalls im Vorfeld der Kategorie von High Potentials zugeordnet wurden, beruht. Aus seiner Studie leitet der Autor drei unterschiedliche Segmente ab: bindungsarme Selbstverwirklicher, anspruchsvolle Allrounder sowie konservative Familienmenschen.

Demgegenüber existiert eine Reihe von Untersuchungen, die sich bei Operationalisierung von Persönlichkeitseigenschaften an den psychologischen Skalen orientieren. Aus der Studie von CABLE und JUDGE (1997) geht beispielsweise ein signifikanter Zusammenhang zwischen den sog. Big Five Persönlichkeitsdimensionen und den bevorzugten Arbeitsplatzmerkmalen hervor (vgl. ähnlich: LIEVENS et al., 2001; SCHREURS et al., 2009). Diese Ergebnisse werden auch durch die Befunde von TURBAN und KEON (1993) gestützt, wonach die beiden persönlichkeitsspezifischen Merkmale Selbstwirksamkeit und Leistungsmotivation die Bewertung von Arbeitsplatzmerkmalen beeinflussen. Darüber hinaus weisen empirische Untersuchungen auf die differenzielle Validität von beruflichen Werthaltungen hin. So konnten etwa JUDGE und BRETZ (1992) zeigen, dass Leistungsorientierung, Fairness und soziale Verantwortung die Organisati- 
onswahl signifikant beeinflussen. Insgesamt wird hier eine Befundlage deutlich, die eine nach persönlichkeitsspezifischen Merkmalen differenzierende Betrachtung nahelegt. Gleichzeitig lässt sich feststellen, dass die zu verwendende Operationalisierungsstrategie einer sorgfältigen Auswahl bedarf, um reliable und valide Ergebnisse zu erzielen.

Die Frage, welche Arbeitsplatzmerkmale eine gewichtige Rolle im Arbeitgeberwahlprozess übernehmen, kann anhand des oben dargestellten Forschungsstands aus mehreren gewichtigen Gründen nicht eindeutig beantwortet werden. So zeigt sich erstens in einer Vielzahl von empirischen Studien zu diesem Thema, dass die Autoren heterogene Arbeitsplatzmerkmale als konstitutiv für die Arbeitgeberwahl erachten. Bei einer näheren Betrachtung lässt sich zugleich feststellen, dass sie aufgrund ihrer abweichenden theoretischen Prämissen und empirischen Erhebungsmethoden nur sehr bedingt miteinander vergleichbar sind. So fehlt größtenteils eine theoriegeleitete Begründung für die Auswahl von berücksichtigten Arbeitsplatzmerkmalen. Ansatzpunkte für eine theoretische Fundierung sind dagegen beispielsweise in solchen Studien erkennbar, in denen Grunddimensionen von Organisationskulturen die Basis für die Bewertung von Arbeitsplatzmerkmalen darstellen (z.B. CABLE und JUDGE, 1996). Wesentliche Unterschiede lassen sich neben der Auswahl der vorgegebenen Arbeitsplatzmerkmale in der Stichprobenzusammensetzung erkennen. NERDINGER (1994) weist in diesem Kontext darauf hin, dass die Befunde nicht nur von den Stichproben abhängig sind, sondern auch von der aktuellen Situation am Arbeitsmarkt und dem Entwicklungsstand des Personalmarketings der Unternehmen. Infolgedessen stellen sich manche Ergebnisse konträr dar, sodass insgesamt von einer stark eingeschränkten Generalisierbarkeit der bisherigen Studien ausgegangen werden kann. Schließlich bleibt in den meisten empirischen Studien völlig unberücksichtigt, dass bei der direkten Befragungsmethodik systematische Urteilstendenzen (sog. Biases) auftreten können, die sich negativ auf die Validität auswirken und infolgedessen auch die Ergebnisinterpretation deutlich erschweren (SATTLER, 2006).

\subsubsection{Praxisorientierte Studien zum Personalmarketing}

Neben den aufgeführten wissenschaftlich fundierten Untersuchungen zum Personalmarketing existiert eine Vielzahl an Veröffentlichungen, die aus primär kommerziellen Interessen umgesetzt wird und auf die Feststellung bzw. Steigerung der Arbeitgeberattraktivität im Sinne des Employer Branding Ansatzes fokussiert (SPONHEUER, 2010). Hierbei werden Unternehmen aus unterschiedlichen Perspektiven, wie beispielsweise aus Sicht von Studierenden bzw. Absolventen oder auch von Personalverantwortlichen, beurteilt und infolgedessen in eine Rangfolge der beliebtesten Arbeitgeber eingestuft. Die Ergebnisse solcher Studien stellen häufig die Grundlage für sog. Employer-Awards dar, die die teilnehmenden Unternehmen ihrem Attraktivitätsniveau bei unterschiedlichen Zielgruppen einordnen (NAUNDORF und SPENGLER, 2012). Weitere Informationen solcher Studien sind u.a. in den von den Zielgruppen bevorzugten Bran- 
chen sowie Arbeitsplatzmerkmalen zu sehen. Zusätzlich zur Veröffentlichung von Ranglisten in einschlägigen Medien werden innerhalb einiger Studien Gütesiegel verliehen, die der Organisation die Erfüllung bestimmter Kriterien bescheinigen und infolgedessen als Arbeitgeber-Auszeichnungen, beispielsweise auf unternehmenseigenen Karriereseiten oder in Stelleninseraten, geführt werden (TAVAKKOLI, 2009).

Obgleich die mediale Präsenz solcher Studien inzwischen vergleichsweise hoch ist, wurde ihre Wirkung hinsichtlich der Arbeitgeberattraktivität oder Bewerbungsbereitschaft bislang nicht hinreichend erforscht. Vielmehr wird von den Auftraggebern dieser Studien, die vor allem in der Medien- und Beratungspraxis zu lokalisieren sind, ad hoc angenommen, dass die veröffentlichten Rankings als Informationsquellen einen bedeutenden Einfluss auf die Wahrnehmung von potenziellen Arbeitgebern durch Studierende und Hochschulabsolventen haben (HUF, 2007). Überdies sollen die an den Studien teilnehmenden Organisationen insofern davon profitieren, als dass die jeweilige Platzierung einen Hinweis auf den Wirkungsgrad ihrer im Personalmarketing eingesetzten Instrumente bietet (SCHERM et al. 2010).

Derartige Studien werden sowohl regional als auch überregional intensiv durchgeführt und breit veröffentlicht (SCHERM und SÜSS, 2010). Dabei weisen die zahlreichen Anbieter aus diesem Bereich sehr unterschiedliche Bekanntheitsgrade auf und sind überdies hinsichtlich der zugrunde liegenden Zielgruppe und Methodik nur bedingt miteinander vergleichbar. Bei einer näheren Betrachtung lassen sich die existierenden Publikationen in drei grobe Richtungen unterteilen (TAVAKKOLI, 2009). Zum einen wird die Arbeitgeberattraktivität in sog. Arbeitgeberwettbewerben mithilfe von Mitarbeiter- und Führungskräftebefragungen sowie externen Beurteilungen ermittelt, wodurch die interne Organisationsperspektive in den Vordergrund rückt. Daneben gibt es Studien, die sich vorrangig mit der Bewertung bzw. dem Ranking durch potenzielle Bewerber und somit durch die Nachfrager auf dem Arbeitsmarkt beschäftigen. Erwähnenswert sind überdies Studien, die einen engen inhaltlichen, regionalen oder zielgruppenspezifischen Fokus haben. Dazu gehören beispielsweise Zertifizierungsleistungen für ausgewählte Arbeitsplatzmerkmale. Im der folgenden Übersicht werden die bekanntesten Anbieter beispielhaft vorgestellt. 


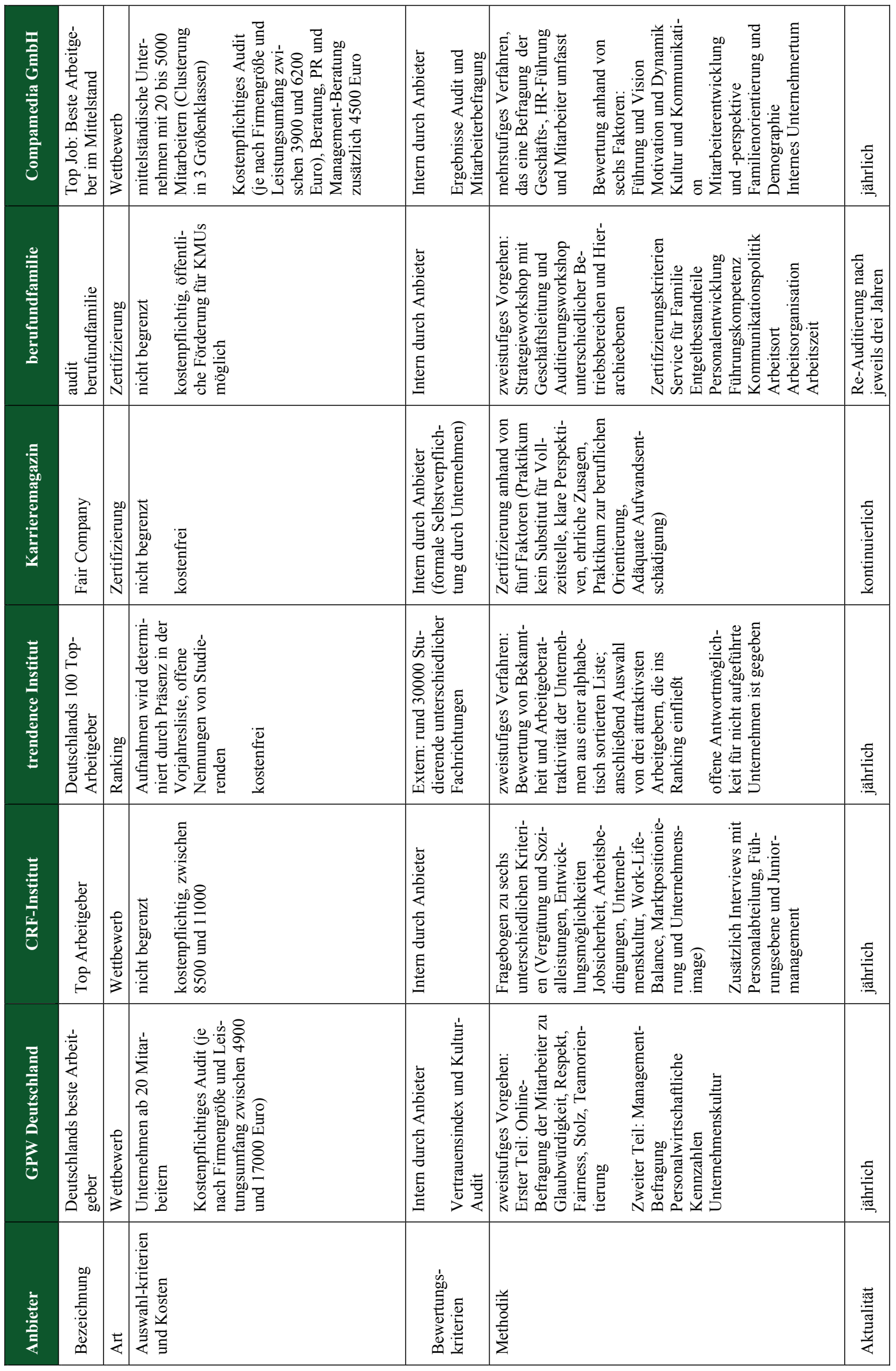

Tab. 2-6: Anbieter von Arbeitgeberwettbewerbern, Zertifizierungen und Rankings

(Eigene Darstellung, 2010) 
Bei einer näheren Analyse der oben dargestellten Studien wird deutlich, dass zwischen den einzelnen Ansätzen grundlegende Unterschiede bestehen. Zum einen liegen deutliche Abweichungen in der Anzahl und Bezeichnung der zugrunde liegenden Dimensionen vor. So basiert die Bewertung eines Arbeitgebers innerhalb der „Great Place to Work“-Studie auf insgesamt fünf Faktoren, wozu Glaubwürdigkeit, Respekt, Fairness, Stolz sowie Teamgeist zählen. Ein ähnlicher inhaltlicher Fokus findet sich in den beiden Studien „Top Arbeitgeber“ und „Top Job: Beste Arbeitgeber im Mittelstand“. Demgegenüber fokussieren andere Studien auf einzelne, eher abgegrenzte Arbeitsplatz- und Organisationsmerkmale, wie beispielsweise die Vereinbarkeit von Beruf und Familie (,,audit berufundfamilie“) oder Fairness im Umgang mit Studierenden und Absolventen bei der Vergabe von Praktikumsplätzen („Fair Company“). Ebenso lassen sich Unterschiede in der methodischen Umsetzung einzelner Studien identifizieren. So umfassen die beiden Studien „Top Job: Beste Arbeitgeber im Mittelstand“ und „Great Place to Work“ eine breit angelegte Mitarbeiterbefragung, während das ,,audit berufundfamilie“ die Mitarbeiterperspektive in einem Auditierungsworkshop mit einer begrenzten Teilnehmerzahl zugrunde legt.

Weiterhin hebt TAVAKKOLI (2009) den Unterschied zwischen den als Wettbewerb und als Ranking konzipierten Studien hervor. So geht er von einer erhöhten Teilnahme an Studien mit einem Wettbewerbsfokus bei weniger bekannten Unternehmen aus besonders personal- und dienstleistungsintensiven Tätigkeitsbereichen aus, die an branchenspezifischen Konkurrenzanalysen (sog. Benchmarks) interessiert sind und überdies nicht hinter den Personalmarketingaktivitäten ihrer direkten Konkurrenten zurückstehen wollen. Infolgedessen werden die Wettbewerbsstudien eher von Branchen wie Pharma/Chemie, IT, Finanzdienstleistungen sowie Gesundheitswesen nachgefragt, während die Ranking-Studien vorrangig die großen Automobilbauer, Unternehmensberatungen, Wirtschaftsprüfungsgesellschaften sowie Konsumgüterhersteller aufführen. Fernerhin bieten Arbeitgeberwettbewerbe speziell für kleine und mittelständische Unternehmen eine Plattform, auf der sie sich ihren potenziellen Bewerbern präsentieren können, da große Unternehmen hierfür eher auf bewährte Instrumente des externen Personalmarketings zurückgreifen. Davon abzugrenzen sind indes die beiden Zertifizierungsstudien „Fair Company“ und „audit berufundfamilie“, da diese zwar ein ähnliches Ziel, d.h. die Erhöhung der Arbeitgeberattraktivität, verfolgen, ihre Ergebnisse aber nicht als Rangfolge teilnehmender Organisationen veröffentlichen. Überdies zeichnet sich die letztgenannte Studie durch einen vergleichsweise hohen Anteil öffentlicher Arbeitgeber aus.

Eine weitere wesentliche Differenzierungsebene zwischen Arbeitgeberwettbewerben und Rankings sieht TAVAKKOLI (2009) in den Adressaten dieser Studien. So nimmt er an, dass Rankings fast ausschließlich von Studierenden, Hochschulabsolventen und Berufseinsteigern wahrgenommen und bei ihrer Bewerbungsentscheidung berücksichtigt werden, während Arbeitgeberwettbewerbe aufgrund ihrer internen Unternehmensperspektive einen größeren Stellenwert bei Bewerbern mit bereits vorhandener Berufserfahrung einnehmen. Allerdings werden zu dieser Annahme keine empirischen Belege angeführt, sodass diese vorerst als hypothetisch zu werten ist. 
Sämtliche Anbieter bewerben die Teilnahme an ihren Studien mit dem Argument, dass die Arbeitgeber mittels der öffentlichkeitswirksamen Prämierungs- und Publikationsleistungen ihren Bekanntheitsgrad ausbauen und sich insbesondere als mittelständisches Unternehmen besser auf dem Arbeitsmarkt positionieren können (NAUNDORF und SPENGLER, 2012). Primäres Ziel dieser Bemühungen besteht folglich in der Optimierung des externen Personalmarketings, d.h. in der Anziehung und Rekrutierung besonders qualifizierter und zur Organisation passender Bewerber (HUF, 2007). Konkrete positive Effekte ergeben sich TAVAKKOLI (2009) zufolge dadurch, dass geschaltete Stellenanzeigen aufgrund des vorhandenen Gütesiegels verstärkt wahrgenommen werden, sodass für die zu besetzenden Stellen insgesamt weniger Anzeigen zu schalten und damit Kostensenkungen zu realisieren wären. Überdies geht der Autor in diesem Zusammenhang von einer erhöhten Anzahl besonders geeigneter Initiativbewerbungen aus. Zudem wird angenommen, dass die Studienergebnisse zentrale Handlungsfelder zur Optimierung der Unternehmensstrategie hinsichtlich der eingesetzten Personalmarketingmaßnahmen offenlegen (STOTZ und WEDEL, 2009).

Obgleich Gütesiegel in der Unternehmenspraxis zunehmend eingesetzt werden, liegen bislang keine empirischen Studien vor, die sich systematisch mit ihrer postulierten Wirkung beschäftigt haben. Die zentrale Annahme, dass die in den Studien verliehenen Gütesiegel die Arbeitgeberwahl potenzieller Bewerber beeinflussen, ist aus wissenschaftlicher Perspektive somit zunächst kritisch zu werten. Ebenso ist aufgrund der bestehenden Intransparenz bei der Gütesiegelvergabe ihre praktische Relevanz zu hinterfragen (SCHERM und SÜSS, 2010; NAUNDORF und SPENGLER, 2012). So wird bei den dazugehörigen Anbietern beispielsweise nach außen nicht deutlich erkennbar, wie die anfängliche Konkurrenzsituation bei einem Arbeitgeberwettbewerb ausgestaltet ist (HuF, 2007). Die zahlreichen Auszeichnungen, Rankings und Gütesiegel bzw. Zertifikate lassen sich zudem nur schwer voneinander abgrenzen, zumal Methodik, Nutzenaspekte, Teilnahmevoraussetzungen und Professionalisierungsgrad einzelner Studien äußerst heterogen gestaltet sind (TAVAKKOLI, 2009). Hinzu kommt, dass die oben aufgeführten Wettbewerbe allesamt kommerziell sind und dadurch a priori eine gewisse Selektion der teilnehmenden Unternehmen hervorbringen. Annehmbar ist, dass sich hieran insbesondere Unternehmen beteiligen, die zwar über einen geringeren Bekanntheitsgrad verfügen, die aufgezeigten Bewertungskriterien jedoch adäquat erfüllen können. Allerdings lässt sich hierbei die Vergleichbarkeit der Unternehmen aufgrund der branchenheterogenen Zusammensetzung deutlich in Frage stellen. So lässt sich ein großes IT-Unternehmen nicht ohne weiteres mit einem mittelständischen lebensmittelproduzierenden Unternehmen vergleichen. Insgesamt lässt sich aufgrund der aufgezeigten Anbietersituation festhalten, dass die angestrebte Informations- und Prestigefunktion von Gütesiegeln auf dem Arbeitsmarkt aufgrund der hohen Anbieteranzahl, bislang fehlender Standardisierungsvorschriften und mangelnder Transparenz zumindest derzeit in Frage zu stellen ist. 
KAPITEL 3

\section{Theoretische Grundlagen der Arbeitgeberwahl}

Im folgenden Abschnitt werden die verschiedenen theoretischen Ansätze zur Erklärung der Arbeitgeberwahl behandelt. Hierzu sind allerdings zwei kurze Vorbemerkungen erforderlich. Die erste gilt dem Gebrauch der Begriffe Theorie und Modell in der einschlägigen Fachliteratur zur Arbeitgeberwahl, worin diese oft recht willkürlich verwendet werden. In den folgenden Ausführungen wird von einer Theorie gesprochen, wenn es sich um die Gesamtheit der Annahmen über den Gegenstandsbereich handelt, und von einem Modell, wenn es um die spezifische Formalisierung einer bestimmten Annahme geht.

Die zweite Vorbemerkung betrifft den Begriff der Arbeitgeberwahl an sich. Während in der englischsprachigen Fachliteratur einheitlich der Begriff ,job choice“ verwendet wird, findet man in den deutschsprachigen Veröffentlichungen zwei Bezeichnungen für den scheinbar gleichen Untersuchungsgegenstand: „Arbeitsplatzwahl“ und „Arbeitgeberwahl“. Beide Begriffe haben zugleich ihre Existenzberechtigung. Aus der ergebnisorientierten Perspektive erscheint der Begriff Arbeitsplatzwahl geeigneter, da Bewerber sich letztlich für einen bestimmten Arbeitsplatz entscheiden und einen entsprechenden Arbeitsvertrag unterschreiben. Die prozessorientierte Betrachtungsweise wird allerdings besser durch den Begriff Arbeitgeberwahl abgebildet, da mehrere Organisationen häufig die gleiche oder eine ähnliche Stellenposition bieten und zugleich die Informationen hierüber steuern. Hinzu kommt, dass Bewerber den eigentlichen fachlichen Tätigkeitsbereich erst nach dem Arbeitsbeginn in vollem Umfang zu beurteilen vermögen. Auch aus der Arbeitgeberperspektive handelt es sich bei der Entscheidung des Bewerbers um eine Arbeitgeberwahl, da sämtliche Personalmarketingaktivitäten darauf abzielen, seine Wahl zugunsten des eigenen Unternehmens zu beeinflussen. Vor diesem Hintergrund wird in den folgenden Ausführungen der Begriff Arbeitgeberwahl verwendet, wobei die Arbeitsplatzwahl hiermit eingeschlossen wird.

Der folgende Forschungsüberblick zur Arbeitgeberwahl beginnt mit den informationsökonomischen Ansätzen, die sich primär mit dem Informationsverhalten potenzieller Bewerber beschäftigen. Darauf folgen die in der psychologischen Forschung weit verbreiteten erwartungs- 
werttheoretischen Ansätze, wobei hier insbesondere die Prämissen zur Alternativenbewertung und -auswahl dargestellt werden. Anschließend werden die prozessorientierten Modelle der Arbeitgeberwahl vorgestellt, in denen postuliert wird, dass sich die Entscheidung für eine Organisation aus mehreren, zeitlich und inhaltlich separaten Phasen zusammensetzt. Aus der kritischen Würdigung der bisherigen Ansätze wird schließlich der bestehende Forschungsbedarf abgeleitet, dem in dieser Arbeit entsprochen werden soll.

\subsection{Determinanten der Arbeitgeberwahl aus informationsökonomischer Sicht}

\subsubsection{Suchkostenansatz}

Zur Erklärung des Informationsverhaltens potenzieller Bewerber werden häufig informationsökonomische Modelle herangezogen. Generell widmet sich die Informationsökonomie, die ein wichtiges Teilgebiet der Institutionenökonomik darstellt, der Verteilung von Informationen unter der Annahme entstehender Informationskosten (STIGLITZ, 2002). Hieraus resultieren die sog. Suchkostenansätze, die auf Situationen mit unvollständiger Information fokussieren und das optimale Suchverhalten modellieren. Die Suchkostenansätze gehen auf die Arbeiten von STIGLER (1961, zit. in TEUFER, 1999) zurück, worin postuliert wird, dass sich die Preise auf unorganisierten Märkten selbst bei gleichen Produkten von Anbieter zu Anbieter unterscheiden. Diese Preisdispersionen werden auf unzureichendes Informationsverhalten des Konsumenten zurückgeführt, das allerdings durch optimales Suchverhalten nach dem niedrigsten Preis kompensiert werden kann. Grundsätzlich wird davon ausgegangen, dass dem Konsumenten sämtliche Anbieter sowie die Wahrscheinlichkeitsverteilung der Preise bekannt sind, was allerdings mit bestimmten Suchkosten in Form von Zeit und Geld einhergeht. Zugleich werden die Suchkosten des Konsumenten für jedes zu prüfende Produktangebot als konstant betrachtet, sodass die Suche nach Preisinformationen stets lohnend ist. Folglich besteht das Entscheidungsproblem darin, vor der Aufnahme des Suchprozesses einen optimalen Suchumfang festzulegen, in dessen Rahmen der Konsument Preisvergleiche durchführt und schließlich das preisgünstigste Angebot annimmt. Somit ist der Nutzen der einzelnen Suchschritte in einer möglichen Verringerung des zu zahlenden Preises zu sehen, dessen Höhe von der Preisdispersion abhängt. Der Suchkostenansatz geht gemäß dem mikroökonomischen Marginalprinzip davon aus, dass der optimale Suchumfang gegeben ist, wenn die Grenzkosten der Informationssuche dem hierdurch ermöglichten Grenzertrag entsprechen. Der Umfang der optimalen Informationssuche wird folglich von der Höhe der Suchkosten und der Wahrscheinlichkeitsverteilung der Preise beeinflusst. Die Anzahl der Suchschritte wird insgesamt dann entsprechend hoch sein, wenn die Preisdispersion groß ist und die Suchkosten niedrig sind. 
Aus der Übertragung des Suchkostenansatzes auf den Arbeitsmarkt gehen die ökonomischen Job-Search-Modelle hervor (STIGLER, 1961, zit. in TEUFER, 1999). Die Informationssuche erstreckt sich zugleich ausschließlich auf das Arbeitsplatzmerkmal Gehalt, das aus einem bestimmten Arbeitsplatzangebot resultiert. Ein Suchschritt entspricht hierbei einem einzelnen Arbeitsplatzangebot, sodass die Informationssuche in der Regel mehrere Arbeitsplatzalternativen umfasst. Gemäß den oben dargestellten Annahmen, wird die optimale Anzahl von Suchschritten anhand der Relation zwischen den Grenzkosten und dem Grenzertrag bestimmt. Für die Arbeitgeberwahl lässt sich hieraus ableiten, dass die Wahrscheinlichkeit für die Teilnahme am Bewerbungsprozess umso höher ist, je geringer die damit einhergehenden Suchkosten sind. Suchkosten können nicht nur finanzieller, sondern auch zeitlicher Art sein, wie sie beispielsweise im Rahmen der Teilnahme an mehrtägigen Auswahlprozessen entstehen. Wie umfangreich der Informationsprozess eines Bewerbers ausfällt, hängt von der Höhe der Suchkosten einerseits und der Bandbreite des relevanten Arbeitsplatzmerkmals andererseits ab. Infolgedessen wird ein potenzieller Bewerber eher dann zu weiteren Suchschritten bereit sein, wenn er beispielsweise eine erhebliche Gehaltsstreuung zwischen den einzelnen Stellenangeboten wahrnimmt.

An dem oben dargestellten Ansatz erscheint insbesondere die zentrale Annahme problematisch, der zufolge die genaue Anzahl der Schritte vor dem Beginn des Suchprozesses vorliegen muss (TEUFER, 1999). Wenn die einzelnen Angebote nicht gleichzeitig, sondern nacheinander eingeholt werden, so könnte bereits nach wenigen Suchschritten der geringste Preis gefunden und der Informationsprozess deshalb abgeschlossen werden. Theoretische Modifikationen begegnen diesem Kritikpunkt mit der Entwicklung sequenzieller Suchmodelle, bei denen nach jedem Suchschritt gesondert über weitere Suchaktivitäten entschieden wird (MCCALL, 1965). Hierbei wird von einem Reservationspreis für ein bestimmtes Produkt ausgegangen, der vor dem Suchbeginn festgelegt und bei dessen Erreichen der Suchprozess abgebrochen wird. Für die Arbeitgeberwahl lässt sich aus dieser Annahme schlussfolgern, dass aus dem Suchverhalten gleichzeitig auch auf das Wahlverhalten der Bewerber geschlossen werden kann (MALMENDIER, 2006). Der Suchprozess wird nämlich dann nicht mehr fortgeführt, wenn ein Stellenangebot mit einem angemessenen Mindestgehalt bzw. Reservationslohn gefunden und als Alternative gewählt wird (vgl. NERDINGER, 1994).

Ein weiterer Kritikpunkt besteht im normativen Charakter der informationsökonomischen Suchkostenansätze (TEUFER, 1999). So wird darin von einem grundsätzlich rational handelnden und entscheidenden Konsumenten ausgegangen, der bestrebt ist, unter Berücksichtigung sämtlicher Suchkosten einen günstigen Kauf zu tätigen. Ein solches Vorgehen stellt jedoch erhebliche und unrealistische Anforderungen an die Kalkulationsfähigkeit und -neigung des Konsumenten, der zusätzlich über umfassende Kenntnisse über die wahre Preis- und Qualitätsverteilung auf dem Markt verfügen muss (WEIBER und ADLER, 1995). Arbeitsmärkte zeichnen sich hingegen dadurch aus, dass Transaktionen, wie beispielsweise Bewerbungen oder Vorstellungsgespräche, aufseiten der Bewerber nur sehr selten getätigt werden. Damit ergibt sich für 
einen potenziellen Bewerber auch nicht die Chance, umfassende Informationen zu erlangen und aus Fehlern in ausreichendem Maße zu lernen, wodurch die hierbei postulierte Rationalität an Märkten nicht einzuhalten ist (MOSER, 1992). Schließlich wird innerhalb der Suchkostenansätze postuliert, dass die Streuung des Gehalts das einzige entscheidungsrelevante Arbeitsplatzmerkmal darstellt, wohingegen der Einfluss weiterer Kriterien völlig unberücksichtigt bleibt (TEUFER, 1999). Dass diese Annahme nicht aufrechtzuerhalten ist, wird vor dem Hintergrund der im Abschnitt 2.5.1 dargestellten wissenschaftlichen Befunde zur Bedeutung von Arbeitsplatzmerkmalen bei der Arbeitgeberwahl besonders deutlich.

\subsubsection{Informationsökonomische Eigenschaftstypologie}

Aus der Kritik an der oben dargestellten Annahme, sämtliche Informationen durch optimales Suchverhalten erlangen zu können, entwickelten sich weiterführende Ansätze, die bei Austauschprozessen von einer eingeschränkten und asymmetrisch verteilten Information ausgehen (vgl. SCHMIDTKE, 2002). Die informationsökonomische Grundannahme besteht hierbei darin, dass in Beschaffungssituationen kundenseitig nur unvollkommene Informationen bezüglich der Qualitäten bzw. der kaufrelevanten Eigenschaften einer Leistung vorliegen, wobei die Konsumenten gleichzeitig über die Möglichkeit verfügen, die daraus resultierende Unsicherheit durch geeignete Informationsmaßnahmen zu reduzieren (WEIBER und ADLER, 1995). So legen DARBY und KARNI (1973, zit. in SCHMIDTKE, 2002) zur Bearbeitung von Absatzmärkten einen Qualitätsunsicherheitsansatz zugrunde, bei dem die Güter nach Art und Ausmaß der Überprüfbarkeit ihrer kaufrelevanten Eigenschaften eingeordnet werden. Obgleich sich die Typologie zunächst lediglich auf Güter bezog, wurde der Begriff später auf Gütereigenschaften ausgeweitet, da die meisten Güter durch Eigenschaften verschiedener Typen gekennzeichnet sein dürften (SCHMIDTKE, 2002). Infolgedessen kann die Informationsasymmetrie in Form von Leistungseigenschaften abgebildet werden, die sich aus den sog. Such-, Erfahrungs- und Vertrauenseigenschaften zusammensetzen (KAAS und BUSCH, 1996). Zwei grundlegende Abgrenzungskriterien dieser Typologie sind die Beurteilbarkeit der Qualitätseigenschaften durch den Konsumenten sowie der Zeitpunkt der Qualitätsbeurteilung.

\begin{tabular}{|c|c|c|c|}
\hline & & \multicolumn{2}{|c|}{ Beurteilungsfähigkeit des Konsumenten } \\
\hline & & vorhanden & nicht vorhanden \\
\hline \multirow{2}{*}{$\begin{array}{l}\text { Zeitpunkt } \\
\text { der Beurteilungs- } \\
\text { möglichkeit }\end{array}$} & vor Kauf & Sucheigenschaften & $\begin{array}{l}\text { Erfahrungs- oder } \\
\text { Vertrauenseigenschaften }\end{array}$ \\
\hline & nach Kauf & Erfahrungseigenschaften & Vertrauenseigenschaften \\
\hline
\end{tabular}

Tab. 3-1: Typologie der Leistungseigenschaften zur Charakterisierung von Kaufentscheidungsprozessen (Eigene Darstellung, 2010, in Anlehnung an WEIBER und ADLER, 1995) 
Die einzelnen Eigenschaftstypen verhalten sich komplementär zueinander, sodass jede Leistung auf der Gesamtbeurteilungsebene immer durch Such-, Erfahrungs- und Vertrauenseigenschaften in unterschiedlichem Ausmaß charakterisiert werden kann, wobei sich deren Anteile zu 100 Prozent ergänzen (WEIBER und ADLER, 1995). In diesem Zusammenhang hat auch der Begriff des informationsökonomischen Dreiecks einen hohen Bekanntheitsgrad in der betriebs- und volkswirtschaftlichen Forschung erreicht (SCHMIDTKE, 2002). Dementsprechend wird auch das Produkt Stellenangebot als ein Bündel von Such-, Erfahrung- und Vertrauenseigenschaften betrachtet, wobei diese mit jeweils unterschiedlichen Suchkosten verbunden sind (GROBE, 2003). Der Qualitätsunsicherheitsansatz kann sich hierbei sowohl auf die Eigenschaften des Arbeitsplatzes als auch auf die Merkmale des potenziellen Arbeitgebers beziehen, worunter insbesondere solche Eigenschaften fallen, die durch den Arbeitgeber zumindest kurzfristig nicht mehr verändert werden können (WEIBER und ADLER, 1995). Allerdings ist die Aufstellung eines allgemeingültigen, festgelegten Schemas für die Einordnung des Produktes Stellenangebot nur eingeschränkt möglich, da die Zuordnung von Leistungseigenschaften zu Such-, Erfahrungs- oder Vertrauenseigenschaften häufig von der Wahrnehmung durch den einzelnen Konsumenten bzw. potenziellen Bewerber abhängt und ferner aus dem situativen Kontext heraus erfolgt (SCHMIDTKE, 2002).

Dennoch werden im Folgenden analog zur informationsökonomischen Literatur je nach Beurteilungsmöglichkeit drei Typen von Leistungseigenschaften eines Stellenangebotes unterschieden, die unterschiedliche Eigenschaftskategorien von Produkten darstellen (GROBE, 2003). So sind Sucheigenschaften dadurch gekennzeichnet, dass sie bereits vor dem Kauf durch den Konsumenten überprüft werden können. Dies gilt beispielsweise für die Gehaltshöhe, die fester Bestandteil des Arbeitsvertrages ist und ferner durch aktuelle Gehaltsstatistiken in einschlägigen Medien relativ zuverlässig und ohne großen Aufwand durch den potenziellen Bewerber eingeschätzt werden kann. Ähnliches gilt für den Standort bzw. die Standorte des in Frage kommenden Arbeitgebers, die direkt erfragt oder eigenständig recherchiert werden können.

Erfahrungseigenschaften können demgegenüber erst nach dem Kauf durch Konsum bzw. Gebrauch beurteilt werden. Dieser Typologie zufolge zählen Arbeitsplatzmerkmale wie Betriebsklima, Weiterbildungsmöglichkeiten oder Arbeitsinhalte zu Erfahrungseigenschaften, da ein Arbeitgeber sich an diese in der Regel nicht vertraglich bindet, sodass die im Vorfeld getroffenen Vereinbarungen erst durch eigene Erfahrungen in der Organisation zu überprüfen sind. Die hierbei anfallenden Suchkosten sind entsprechend höher einzustufen, da persönliche Erfahrungen mit dem potentiellen Arbeitgeber, z.B. im Rahmen eines Praktikums, dazu erforderlich sind.

Anders verhält es sich mit den Vertrauenseigenschaften, die der Konsument im Gegensatz zu den Such- und Erfahrungseigenschaften weder vor noch nach dem Kauf vollständig beurteilen kann. Dieser Umstand lässt sich einerseits auf das Unvermögen des Konsumenten zurückfüh- 
ren, da ihm das zur Beurteilung notwendige Wissen nicht zur Verfügung steht. Andererseits kann der Beurteilungsaufwand ein inakzeptables Ausmaß annehmen (SCHMIDTKE, 2002). Demnach gehören Arbeitsplatzmerkmale wie beispielsweise Arbeitsplatzsicherheit oder die Aufstiegschancen zu den Vertrauenseigenschaften, da sie letztlich nur im Laufe der eigenen beruflichen Laufbahn zu prüfen sind (GROBE, 2003). Auch bestimmte Arbeitgeberimages, beispielsweise ein besonders fairer Arbeitgeber im Umgang mit Studierenden und Absolventen zu sein, gehören zu diesen Leistungseigenschaften (TEUFER, 1999). In der folgenden Übersicht werden ausgewählte Arbeitsplatzmerkmale gemäß ihrer Leistungseigenschaften typologisiert. Die hier vorgenommene Betrachtung fokussiert allerdings eindeutig auf den Informationsstand eines Entscheidungsträgers zu einem Zeitpunkt, bevor die eigentliche Bewerbungsentscheidung getroffen wurde, sodass die hierbei als Vertrauenseigenschaften gekennzeichneten Arbeitsplatzmerkmale sich in weiterem Verlauf als Erfahrungseigenschaften herausstellen können, z.B. nach Ablauf der Probezeit.

\begin{tabular}{|l|l|l|}
\hline Sucheigenschaften & Gehaltsprogression & Aufstiegschancen \\
\hline Einstiegsgehalt & Arbeitsklima & Sicherer Arbeitsplatz \\
\hline Sozialleistungen & $\begin{array}{l}\text { Schnelle Verantwortungs- } \\
\text { übernahme }\end{array}$ & Zukunftsfähigkeit der Branche \\
\hline Standort & Weiterbildung & Organisationskultur \\
\hline Urlaubsanspruch & & $\begin{array}{l}\text { Gesellschaftliche Unternehmens- } \\
\text { verantwortung (CSR) }\end{array}$ \\
\hline Kinderbetreuung & Reisetätigkeit & \\
\hline \multicolumn{2}{|c|}{ flexible/geregelte Arbeitszeiten } & \\
\hline \multicolumn{2}{|c|}{ Renschen } \\
\hline
\end{tabular}

Tab. 3-2: Such-, Erfahrungs- und Vertrauenseigenschaften eines Stellenangebotes

(Eigene Darstellung, 2010, in Anlehnung an GROBE, 2003)

Aus der obigen Typologie des Produktes Stellenangebot geht zugleich hervor, dass dieses überwiegend durch Erfahrungs- und Vertrauenseigenschaften und weniger durch Sucheigenschaften gekennzeichnet ist (vgl. SCHMIDTKE, 2001). Hieraus lässt sich wiederum ein hohes wahrgenommenes Kaufrisiko ableiten und die Arbeitgeberwahl aus eine Entscheidung unter Unsicherheit charakterisieren (BOSWELL et al., 2003). Da der Erklärungsbeitrag der Suchkostenansätze lediglich auf die Sucheigenschaften beschränkt ist, wird im Folgenden die Signaling-Theorie vorgestellt, aus der sich unsicherheitsreduzierende Effekte bei der Beurteilung von Erfahrungsund Vertrauenseigenschaften ableiten lassen. 


\subsubsection{Signaling-Theorie}

Die Theorie des wahrgenommenen Risikos postuliert für Kaufentscheidungsprozesse, dass die unvollständigen Informationen zwar zur Unsicherheit des Konsumenten führen, diese allerdings erst dann als Risiko wahrgenommen wird, wenn die Entscheidungssituation selbst oder ihre Konsequenzen als subjektiv bedeutend eingestuft werden (KROEBER-RIEL et al., 2009). Grundsätzlich lassen sich zwei Arten von Unsicherheit unterscheiden (NIEDERAUER, 2009). Die Marktunsicherheit resultiert endogen aus dem Verhalten der Marktpartner, während die Umweltunsicherheit exogen gegeben ist und somit durch die Marktteilnehmer nicht beeinflussbar ist. Im ersten Fall reagieren Konsumenten folglich nicht mehr nur passiv auf eine gegebene Unsicherheitssituation, sondern können durch Informationsaufnahme ihre Marktunsicherheit reduzieren. Im Rahmen der Informationsökonomie wird ausschließlich die endogen verursachte Ungleichverteilung von Informationen analysiert. Allerdings ist der Abbau von Wissensdefiziten stets mit Informationskosten verbunden, sodass die Unsicherheit nicht vollständig beseitigt werden kann.

Wie oben dargestellt, ist die Arbeitgeberwahl zunächst durch eine deutliche Informationsasymmetrie zugunsten der Unternehmen als Arbeitgeber gekennzeichnet, deren Verringerung mit hohen Suchkosten für den potenziellen Bewerber verbunden ist (SCHMIDTKE, 2002). Die Übertragung der Kaufentscheidungssituation auf die Arbeitgeberwahl führt zu mehreren Ableitungen. So kann zum einen das empfundene Risiko eines potenziellen Bewerbers bei seiner Entscheidungsfindung als hoch eingestuft werden, da das Maß der ihm zur Verfügung stehenden Informationen zunächst gering ist (ALLEN et al., 2007). Dabei sind vor allem die Tragweite und geringe Reversibilität der Entscheidung als ursächlich für diesen Umstand zu nennen (TEUFER, 1999). Zudem ist zu beachten, dass mit zunehmender Entscheidungsnähe das Risikoempfinden ansteigt, wodurch auch die Motivation des potenziellen Bewerbers, zusätzliche Informationen zu suchen, größer wird (KROEBER-RIEL et al., 2009). Die Risikominimierung kann hierbei mittels unterschiedlicher Informationsstrategien vollzogen werden, wobei es sich bei der Arbeitgeberwahl um einen Fall der Verhaltensunsicherheit handelt, die mithilfe von sog. Screening oder Signaling überwunden werden kann (RILEY, 2001). Während das Signaling sämtliche Informationsaktivitäten umfasst, die von der besser informierten Seite ausgehen und an die schlechter informierte Marktseite gerichtet sind, zählen zum Begriff des Screenings alle Informationsbeschaffungsaktivitäten der schlechter informierten Seite.

Unter Bezugnahme auf das zentrale Forschungsziel dieser Arbeit ist vor allem die Frage interessant, wie Unternehmen die Bewerbungsentscheidung geeigneter und qualifizierter Studierender und Absolventen beeinflussen können. Im Vordergrund der weiteren Ausführungen soll daher die Wahrnehmung und Wirkung von Signalen der Arbeitgeber auf potenzielle Bewerber stehen. In der wissenschaftlichen Literatur zur Arbeitgeberwahl nimmt die Signaling-Theorie eine stetig wachsende Bedeutung ein, wenngleich weiterführende Forschungsaktivitäten auf 
diesem Gebiet eingefordert werden (EHRHART und ZIEGERT, 2005; ALLEN et el., 2007; BOSWELL et al., 2012). Generell stellt die Signaling-Theorie eine informationsökonomische Forschungsrichtung dar, die sich mit den Interpretationsmöglichkeiten von Signalen und den daraus abzuleitenden Schlussfolgerungen für die Vertragsgestaltung beschäftigt (SPENCE, 1973, 2002; CONNELY et al., 2011). Bei Signalen handelt es sich um beobachtbare Eigenschaften des Signalsenders, aufgrund derer der Signalempfänger unter genau definierbaren Bedingungen vor dem Vertragsschluss auf nicht beobachtbare Eigenschaften des Senders zurückschließen kann. Signale werden hierbei zugleich als Instrumente definiert, die aufgrund ihrer Beschaffenheit die Informationsasymmetrie verringern können. Eine wesentliche Annahme besteht hierbei darin, dass Signale dem Konsumenten als sog. Information Chunks in komprimierter Form Informationen über schwer oder nicht zu beurteilbare Leistungseigenschaften, also Erfahrungs- und Vertrauenseigenschaften, liefern (NIEDERAUER, 2009). Anders ausgedrückt, werden mithilfe von Signalen solche Informationen ersetzt und gebündelt, die andernfalls zur Beurteilung von Leistungseigenschaften herangezogen worden wären. Eine wesentliche Funktion von Signalen ist somit darin zu sehen, die Unsicherheit von Konsumenten zu reduzieren, indem sie diese als Indikatoren für nicht beobachtbare Leistungseigenschaften verwenden (SAMMER und WÜSTENHAGEN, 2006).

Die Wirksamkeit von Signalen hängt zugleich im Wesentlichen davon ab, inwieweit sie vom Konsumenten als subjektiv geeignete Informationsgrundlage zur indirekten Beurteilung der zum Zeitpunkt der Kaufentscheidung unsicheren Leistungseigenschaften wahrgenommen werden. Das Verhältnis zwischen dem Anbieter und dem Konsumenten lässt sich aus informationsökonomischer Sicht als wechselseitig kennzeichnen, da der Konsument auf die vom Anbieter zur Verfügung gestellten Informationen angewiesen ist (SPENCE, 2002). Aus der Signalübermittlung entstehen allerdings Kosten, die unabhängig von der Reaktion des Konsumenten auf diese Signale sind. Folglich entsendet ein Anbieter kostenverursachende Signale, die jedoch in keiner expliziten vertraglichen Regelung zum Konsumenten stehen.

Obgleich die informationsökonomische Forschung vorwiegend Informationsasymmetrien auf Seiten der Arbeitgeber, wie beispielsweise nicht beobachtbare produktivitätsrelevante Eigenschaften von potenziellen Bewerbern, untersucht, sind auch die letztgenannten ungleich verteilter Information ausgesetzt (SCHMIDTKE, 2002). Hinsichtlich der Arbeitgeberwahl wird dabei postuliert, dass potenzielle Bewerber grundsätzlich daran interessiert sind, möglichst viele Informationen über die tatsächliche Qualität ihres zukünftigen Arbeitsplatzes für ihre Bewerbungsentscheidung heranzuziehen (ALLEN et el., 2007). Weiterhin wird davon ausgegangen, dass die am Arbeitsmarkt existierenden Informationsasymmetrien mithilfe von Signalen reduziert werden können (SCHMIDTKE, 2002). So können beispielsweise aufseiten der Bewerber Unsicherheiten über nicht direkt beobachtbare Arbeitsplatzmerkmale, wie beispielsweise Betriebsklima, Arbeitszeiten oder Weiterbildungsmöglichkeiten, vorherrschen. Da potenzielle Bewerber die Arbeitsplatzmerkmale im Gegensatz zu den Arbeitgebern nicht direkt beobachten können, liegt 
hier eine Informationsasymmetrie zugunsten der Arbeitgeber vor. Um diese Informationsasymmetrie zu reduzieren, versuchen Bewerber weitere vorhandene Informationen heranzuziehen oder auf Signale zurückzugreifen (BOSWELL et al., 2003). Zugleich haben die Unternehmen ein Interesse daran, glaubhafte Informationen über sich zu vermitteln (CHAPMAN und WEBSTER, 2006). Signale dienen hierbei dazu, die Informationsasymmetrie aufseiten der potenziellen Bewerber zu überwinden. Die Arbeitgeber nutzen sie demgemäß dazu, unbeobachtbare Arbeitsplatzmerkmale zu kommunizieren, um auf diese Weise die Bewerbungsentscheidung zu erleichtern (SCHMIDTKE, 2002). Damit eine Eigenschaft jedoch überhaupt zum Signal werden kann, muss sie vorher durch den Signalsender verändert bzw. gestaltet werden (NIEDERAUER, 2009). Dem Signaling-Ansatz liegt dabei die Annahme zugrunde, dass potenzielle Bewerber lediglich Informationen über beobachtbare Arbeitsplatzmerkmale als glaubhafte Kommunikation wahrnehmen, während unbeobachtbare Arbeitsplatzmerkmale grundsätzlich einem Glaubwürdigkeitsproblem unterliegen, da positive Informationen hierbei leicht hervorgehoben und negative entsprechend verdeckt werden können (ALLEN et el., 2007). Signale werden hingegen deshalb als relativ glaubwürdig wahrgenommen, da die Kosten der Signalerzeugung negativ mit der Ausprägung nicht beobachtbarer Arbeitsplatzmerkmale korrelieren (SCHMIDTKE, 2002). Daraus folgt, dass Unternehmen, in denen das nicht beobachtbare Arbeitsplatzmerkmal nicht oder nur in geringer Ausprägung vorliegt, höhere Kosten in die Signalwirkung investieren müssten, als Unternehmen mit einer hohen Ausprägung des nicht beobachtbaren Arbeitsplatzmerkmals. Diese Kosten verhindern zugleich, dass dieselben Signale von schlechter aufgestellten Anbietern verwendet werden, da es sich für diese nicht lohnen würde, Kosten zur Übermittlung eines gar nicht oder nur geringer ausgeprägten Arbeitsplatzmerkmals aufzuwenden, auch wenn hierdurch der Rekrutierungserfolg steigen würde. Infolgedessen kann ein potenzieller Bewerber hieraus zuverlässige Rückschlüsse hinsichtlich der für ihn relevanten Arbeitsplatzmerkmale ziehen.

In den bisherigen Ausführungen wurde das Produkt Stellenangebot als ein Gut charakterisiert, bei dem Erfahrungs- und Vertrauenseigenschaften dominieren (TEUFER, 1999; GROBE, 2003). Je schwieriger es somit ist, die Leistungseigenschaften eines Produktes, aufsteigend von Suchüber Erfahrungs- zu Vertrauenseigenschaften, zu beurteilen, umso mehr steigt auch das wahrgenommene Kaufrisiko (KAAS und BUSCH, 1996). Infolgedessen werden zur Qualitätsbeurteilung zunehmend Signale herangezogen, die als Informationsbündel bzw. -ersatz verlässliche Hinweise auf die zu erwartenden Leistungseigenschaften geben und dadurch zugleich das Kaufrisiko zumindest teilweise vermindern. Zu solchen Signalen zählen sog. Stellvertreter-Informationen oder Information Chunks, die als Schlüsselinformationen für andere Indikatoren herangezogen werden (TROMMSDORFF, 2011). Im Konsumgüterbereich werden darunter Markennamen, Gütesiegel, Branchenimages, Referenzen bzw. Empfehlungen und Testurteile gefasst (BURMANN und SCHAEFER, 2005). 
Die besondere Wirkungsweise der Gütesiegel lässt sich auf dieser Basis generell dadurch erklären, dass sie eine Vermittlerfunktion im Vertrauensbildungsprozess zwischen einem Konsumenten und einem bisher nicht oder nur wenig bekanntem Anbieter einnehmen, indem sie als eine dritte, unabhängige und daher auch vertrauenswürdige Partei fungieren (NOLL und WINKLER, 2004). Gütesiegel implizieren die Einhaltung vorher definierter Qualitätsmindestanforderungen bzw. Prüfkriterien, wobei die Qualitätsaussage durch anbieterunabhängige Instanzen getroffen wird (SPILLER, 2001). Die hiermit einhergehende Selbstbindung und -verpflichtung kann insofern zum Vertrauensaufbau führen, als dass das bislang fehlende Vertrauen in den Anbieter durch das Vertrauen in die unabhängige Expertenorganisation ersetzt wird (NOLL und WINKLER, 2004). Dementsprechend konnte im Konsumgüterbereich gezeigt werden, dass die Bedeutung von Stellvertreter-Informationen zunimmt, wenn das wahrgenommene Kaufrisiko und damit gleichzeitig der Anteil von Erfahrungs- und Vertrauenseigenschaften steigen (BURMANN und SCHAEFER, 2005). Allerdings existieren bislang nur wenige Forschungsarbeiten, die solche Erkenntnisse auf die Frage der Arbeitgeberwahl übertragen (BosWELL et al., 2012). Aufgrund der Annahmen der Signaling-Thorie und der bereits vorliegenden empirischen Befunde kann jedoch insgesamt davon ausgegangen werden, dass die Bewerbungsentscheidung vor allem zu Beginn des Personalmarketingprozesses auf der Grundlage von Stellvertreter-Informationen und Assoziationen getroffen wird (vgl. z.B. RYNES, 1991; GATEWOOD et al., 1993; TURBAN und Greening, 1996; CABle und Turban, 2001; HighHOuse et al., 2007; VON WALTER et al., 2011). Infolgedessen kann hierbei angenommen werden, dass potenzielle Bewerber neben direkt beobachtbaren Arbeitsplatzmerkmalen auch auf verfügbare Signale zur Beurteilung von nicht beobachtbaren Arbeitsplatzmerkmalen eines Stellenangebotes zurückgreifen (SCHMIDTKE, 2002). In diesem Kontext zeigt sich eine inhaltliche Überschneidung zu dem oben vorgestellten ELM-Modell von PETTY UND CACIOPPO (1986), das bislang nicht systematisch auf die informationsökonomische Gütertypologie angewendet wurde. Da die Erfahrungs- und Vertrauenseigenschaften des Produktes Stellenangebot von potenziellen Bewerbern zunächst nur eingeschränkt beurteilt werden können, sollte hier eher die periphere Route der Informationsverarbeitung gewählt werden, was wiederum einen höheren Einfluss von Signalen, wie beispielsweise Gütesiegeln, vermuten lässt (BURMANN und SCHAEFER, 2005).

Innerhalb des Unsicherheitsansatzes nimmt ferner das Konstrukt der Reputation einen hohen Stellenwert ein (WIEDMANN et al., 2006). Neben dem Marketing greifen auch andere Forschungsdisziplinen, wie beispielsweise die Soziologie, auf dieses Konstrukt zurück, woraus die Reputation und ihr Synonym, der Ruf, allerdings uneinheitliche und teils kontroverse Begriffsdefinitionen erfahren (siehe für einen Überblick: ALBERSMEIER und SPILLER, 2009). Ungeachtet der multidisziplinär bedingten Definitionsunterschiede, wird hierunter generell der „Ruf respektive die Anerkennung einer Person oder Organisation durch außenstehende Dritte" verstanden (EISENEGGER und KÜNSTLE, 2003: 58). Das zugrundeliegende Abhängigkeitsverhältnis von Dritten bedeutet zugleich, dass es sich bei der Reputation um ein grundsätzlich erwerbbares Gut handelt, das auch wieder abgesprochen werden kann und daher einer kontinuierlichen Pflege bedarf. 
EISENEGGER und IMHOF (2009) zufolge kann die Reputation, verstanden als der Ruf oder das Ansehehen eines Anbieters am Markt, als das zentrale Signal zur Unsicherheitsreduktion aufgrund von Informationsasymmetrien betrachtet werden, das sich generell aus extrapolierten Erfahrungen zusammensetzt, die der Konsument allerdings nicht selbst gemacht haben muss. Die bisher gezeigten Leistungen stellen dabei einen verlässlichen Hinweis für die zukünftigen Qualitätseigenschaften dar. Die Reputation dient somit als Stellvertreter-Information im Beurteilungsprozess eines Konsumenten und kommt besonders dann zum Tragen, wenn das wahrgenommene Risiko hoch ist (NIEDERAUER, 2009). Dabei ist die Reputation definitorisch von dem durchaus ähnlichen Begriff des Images abzugrenzen, was in der Literatur bislang noch nicht durchgängig gelingt (GOTSI und WILSON, 2001). So werden nicht nur in der praxisorientierten, sondern auch in der marketingwissenschaftlichen Literatur zur Arbeitgeberwahl die beiden Begrifflichkeiten miteinander vermischt oder gar gleichgesetzt. Das Image lässt sich dabei insofern von der Reputation einer Organisation abgrenzen, als dass das Image primär ein neutrales Bild umfasst, während die Reputation sich aus der Bewertung des Images zusammensetzt und somit aus den evaluativen Assoziationen gegenüber einem Arbeitgeber gebildet wird (VON WALTER et al., 2009). Die Reputation eines Unternehmens kann zudem insofern von seinem Image abgegrenzt werden, ,,als sich hierin zugleich die in der Vergangenheit aufgebauten und für die Zukunft relevanten Unterstützungspotenziale manifestieren“ (WIEDMANN et al., 2006: 99). Zu solchen Unterstützungspotenzialen zählen demgemäß beispielsweise das Vertrauen und die Glaubwürdigkeit eines Unternehmens, aber auch das Ausmaß an Aufmerksamkeit und Interesse, das ihm entgegengebracht wird.

Analog dazu werden die beiden Begriffe Vertrauen und Reputation häufig nicht klar voneinander abgegrenzt. EISENEGGER und IMHOF (2009) präzisieren das mit der Reputation einhergehende Vertrauen, indem sie hervorheben, dass der Anbieter dem Konsumenten vor einer Transaktion häufig unbekannt ist und das Vertrauen somit nicht ausschließlich auf persönlichen Erfahrungen, sondern auch auf indirekten Informationen Dritter beruhen kann. Reputation stellt indes nicht nur die Voraussetzung für die Entwicklung des Vertrauens dar, auch Vertrauen bildet die Voraussetzung für Reputationsbildung, sodass diese beiden Konstrukte als interdependent angesehen werden können. EISENEGGER und KÜNSTLE (2003) verdeutlichen diesen Zusammenhang, indem sie zwischen der funktionalen und sozialen Reputation unterscheiden. Während die funktionale Reputation daran gemessen wird, wie gut der Reputationsträger seinen Leistungsauftrag erfüllt hat, liegt der sozialen Reputation der Bewertungsmaßstab zugrunde, inwieweit hierbei gesellschaftliche Normen und Werte berücksichtigt wurden. Demzufolge bildet sich Vertrauen aus, wenn die Erwartungen relevanter Bezugsgruppen sowohl in funktionaler als auch in sozialer Hinsicht erfüllt werden. Der Zusammenhang lässt sich nun so skizzieren, dass die erfüllten Erwartungen zu positiven Zukunftserwartungen und zu Vertrauen führen, aus denen sich die Reputation herausbildet. 
Hinsichtlich der Arbeitgeberwahl lässt sich hieraus ableiten, dass die Reputation eines Arbeitgebers einen entscheidenden Wettbewerbsvorteil auf dem Arbeitsmarkt darstellen kann, da sie die Entscheidungsunsicherheit von potenziellen Bewerbern zu reduzieren vermag (WILLIAMSON et al., 2010; CONNELY et al., 2011). Ihr Wirkmechanismus als Signal entfaltet sich vor allem in solchen Entscheidungssituationen, in denen zwischen dem Arbeitgeber und dem potenziellen Bewerber noch kein persönlicher Kontakt stattgefunden hat (HAUTZINGER, 2009). Die positiven Effekte einer intakten Unternehmensreputation sind folglich darin zu sehen, dass ein Arbeitgeber die Erfahrungs- und Vertrauenseigenschaften aufgrund des bestehendes Vertrauens glaubwürdig kommunizieren und zugleich das wahrgenommene Risiko eines potenziellen Bewerbers verringern kann (SCHMIDTKE, 2002). Allerdings ist die Reputation nur dann als Mittel zur Unsicherheitsreduktion geeignet, wenn bereits eine gute vorliegt (EISENEGGER und IMHOF, 2009). Dieser Umstand erweist sich insbesondere für die kleinen und mittelständischen Unternehmen als problematisch, da sie aufgrund ihres i.d.R. geringen Bekanntheitsgrades häufig über keine ausgeprägte und von außen gut wahrnehmbare Unternehmensreputation verfügen (SCHMIDTKE, 2001). Um die postulierte Signalwirkung innerhalb der Arbeitgeberwahl dennoch untersuchen können, wird hierbei auf die nächsthöhere Ebene, die Branchenreputation der Ernährungsindustrie zurückgegriffen.

\subsection{Determinanten der Arbeitgeberwahl aus erwartungswerttheoretischer Sicht}

Innerhalb der psychologischen Studien zur Arbeitgeberwahl stellt VROOMS (1966) Valenz-Instrumentalitäts-Erwartungs-Theorie (VIE-Theorie) die bekannteste und am häufigsten getestete Theorie dar. Generell wird gemäß dem erwartungswerttheoretischen Ansatz bei der Wahl zwischen mehreren Handlungsalternativen jene bevorzugt, bei der das Produkt aus erzielbarem Wert (Anreiz) und der Wahrscheinlichkeit, diesen tatsächlich auch zu erzielen (Erwartung), maximal ist (BECKMANN und HECKHAUSEN, 2010). Es wird somit ein Zielzustand angestrebt, der einen möglichst hohen Anreizwert hat. Vor diesem Hintergrund liegt den Erwartungs-malWert-Theorien häufig ein hedonistisches Nutzenkalkül zugrunde (NERDINGER, 1994). Hinsichtlich der Arbeitgeberwahl postuliert die VIE-Theorie, dass die Bewerbungsbereitschaft anhand der Bewerbererwartungen und der dabei eingeschätzten Fähigkeit des Arbeitsgebers, diese Erwartungen auch erfüllen zu können, erklärt werden kann. Demzufolge wird zunächst die Valenz, d.h. der Wert oder die Anziehungskraft eines bestimmten Stellenangebotes auf folgende Weise bestimmt: Sie stellt eine Funktion der summierten Produkte der Valenzen der einzelnen Arbeitsplatzmerkmale und der wahrgenommenen Instrumentalitäten eines bestimmten Stellenangebotes zur Realisierung dieser Arbeitsplatzmerkmale dar. 
Hieraus wird die folgende Formalisierung abgeleitet (vgl. NERDINGER, 1994):

$$
V_{j}=f\left[\sum_{k=1}^{n}\left(V_{k} \times I_{j k}\right)\right]
$$

Dabei bedeuten:

$\mathrm{V}_{\mathrm{j}} \quad=$ Valenz eines bestimmten Stellenangebotes $\mathrm{j}$

$\mathrm{V}_{\mathrm{k}} \quad=$ Valenz des Arbeitsplatzmerkmals $\mathrm{k}$

$\mathrm{I}_{\mathrm{jk}} \quad=$ Instrumentalität des Stellenangebotes j, um die Arbeitsplatzmerkmale k zu realisieren.

Demgemäß ist die Valenz ( Vj) eines bestimmten Stellenangebotes $\mathrm{j}$ eine Funktion der summierten Produkte der Valenzen (Vk) der einzelnen Arbeitsplatzmerkmale des Angebotes und der wahrgenommenen Instrumentalitäten (Ijk) des Stellenangebotes $\mathrm{j}$ für die Realisierung dieser Arbeitsplatzmerkmale. Somit basiert die Attraktivitätsbeurteilung einer Organisation durch potenzielle Bewerber darauf, dass anfangs eine bestimmte Anzahl von Arbeitsplatzmerkmalen spezifiziert und in ihrer Wertigkeit miteinander verglichen wird. Anschließend wird für jedes einzelne Arbeitsplatzmerkmal geprüft, inwieweit dieses innerhalb des vorliegenden Stellenangebotes zu realisieren ist. Zugleich lässt sich die Attraktivität eines bestimmten Stellenangebotes ausschließlich anhand seiner relativen Bedeutung in Bezug auf andere Stellenangebote festlegen, wozu die simultane Bestimmung der Valenz eines oder mehrerer zusätzlicher Angebote erforderlich ist. Die simultane Abwägung impliziert zugleich die Annahme, dass weniger bedeutsame Arbeitsplatzmerkmale durch solche mit einer hohen Relevanz kompensiert werden können. Infolgedessen lässt sich das Stellenangebot mit der höchsten Valenz auch als das attraktivste für potenzielle Bewerber kennzeichnen. Aus der Attraktivitätseinschätzung allein geht allerdings noch nicht die Entscheidung für ein bestimmtes Stellenangebot hervor. Hierzu bedarf es der Bestimmung der Erwartung (Ej), worunter die subjektive Wahrscheinlichkeit, eine ausgeschriebene Stelle zu erhalten, verstanden wird. Daraus resultiert schließlich die Tendenz (Fj), ein bestimmtes Stellenangebot zu wählen, die häufig als Bewerbungsbereitschaft bezeichnet wird. Formalisiert lässt sich das vollständige Modell folgendermaßen darstellen (vgl. NERDINGER, 1994):

$$
F_{j}=f\left[\sum_{k=1}^{n}\left(V_{k} \times I_{j k}\right) E_{j}\right]
$$

Dabei bedeuten:

$\mathrm{F}_{\mathrm{j}} \quad=$ Tendenz, das Stellenangebot $\mathrm{j}$ zu wählen

$\mathrm{E}_{\mathrm{j}} \quad=$ subjektive Wahrscheinlichkeit eines Stellenangebotes

$\mathrm{V}_{\mathrm{k}} \quad=$ Valenz des Arbeitsplatzmerkmals $\mathrm{k}$

$\mathrm{I}_{\mathrm{jk}} \quad=$ Instrumentalität des Stellenangebotes j, um die Arbeitsplatzmerkmale k zu realisieren. 
Aus der VIE-Theorie ergibt sich insgesamt ein eindeutig operationalisierbares und überprüfbares Modell der Arbeitgeberwahl, dem zufolge sich potenzielle Bewerber für diejenige Alternative entscheiden, die ein positives Ergebnis erwarten lässt und gleichzeitig einen hohen persönlichen Wert hat (MOSER und ZEMPEL, 2006). Empirisch konnten die Modellannahmen bislang aber nur mäßig bestätigt werden, wie die Metaanalyse von VAN EERDE und THIERRY (1996) zeigt. So haben die Autoren vergleichsweise viele Studien gefunden, in denen die Valenz bzw. Attraktivität als Prädiktor für entscheidungsrelevante Kriterien erhoben wird. Daraus geht eine durchschnittliche Korrelation mit der direkt erhobenen Arbeitgeberpräferenz von .66 hervor, was allerdings lediglich als partielle Validierung des zugrunde liegenden Modells verstanden werden kann. Denn gleichzeitig stellten die Autoren fest, dass die Valenz über die Instrumentalität hinaus keinen Beitrag zur Vorhersage der Wahlentscheidung leistet, wodurch die Gültigkeit der VIETheorie kritisch zu werten ist. Darüber hinaus finden sich in der Literatur weitere methodische Kritikpunkte, die bei Frage zur Anwendbarkeit der Theorie im Kontext der Arbeitgeberwahl zu bedenken sind (MOSER und ZEMPEL, 2004, und die darin zitierte Literatur):

- Das theoretische Konstrukt der Erwartung wurde in der bisherigen Forschung unzureichend operationalisiert, da der Begriff der subjektiven Wahrscheinlichkeit für viele befragte Personen nicht verständlich formuliert war.

- Realistische Entscheidungssituationen gehen mit einer Varianzeinschränkung bei einigen Variablen einher. So werden bei einer Erfolgswahrscheinlichkeit nahe Null bestimmte Organisationen praktisch von vornherein nicht zur Entscheidung herangezogen. Hieraus resultiert eine verminderte externe Validität der in den Laborexperimenten gewonnenen Ergebnisse.

- Zwischen den beiden Konstrukten Erwartung und Attraktivität besteht eine hohe Korrelation. Zugleich wird die Annahme, dass potenzielle Bewerber während des Bewerbungsprozesses Erwartungen und Valenzen multiplikativ kombinieren, anhand der vorliegenden Forschungsergebnisse nicht gestützt. Im Vergleich zum multiplikativen Ansatz sind die additiven Modelle besser zur Integration von Erwartung und Valenz bzw. Attraktivität geeignet.

- In der VIE-Theorie wird von einem simultanen Vergleich mehrerer Stellenangebote ausgegangen, wobei verschiedene Arbeitsplatzmerkmale in kompensatorischer Weise miteinander verrechnet werden. Gegen diese Annahme sprechen allerdings empirische Befunde, denen zufolge potenzielle Bewerber eher eine sequentielle Entscheidungsstrategie verfolgen und die Annehmbarkeit bestimmter Stellenangebote entsprechend nacheinander prüfen. Die Entscheidung ist hierbei davon abhängig, ob sogenannte Minimalstandards, d.h. Grundansprüche potenzieller Bewerber, überschritten werden. 
- Die VIE-Theorie fasst die Entscheidung als einen rationalen Prozess der Arbeitgeberwahl auf. Allerdings wird diese Annahme nicht theoretisch hergeleitet, sondern vielmehr aufgrund der hierzu vorhandenen Forschungsmethodik vorausgesetzt. Dies führt $\mathrm{zu}$ einer Konfundierung der Forschungsmethodologie mit der theoretisch fundierten Orientierung.

- Ebenso unterstellt die Theorie potenziellen Bewerbern eine vollständige Elaboration und Integration sämtlicher Valenz-, Instrumentalitäts- und Erwartungskomponenten, wodurch sie ein uneingeschränktes und damit unrealistisches Informationsverhalten voraussetzt.

- Ferner lässt sich daraus nicht ableiten, welche Stellenangebote potenzielle Bewerber für sich als grundsätzlich annehmbar betrachten. Unternehmen sind jedoch häufig gerade an der Frage interessiert, ob sie als potenzielle Arbeitgeber überhaupt in Erwägung gezogen werden.

- Schließlich ist die VIE-Theorie indifferent gegenüber der Frage, worin genau der Aufwand bzw. die Kosten einer Bewerbungsentscheidung, wie sie beispielsweise in umfassenden Informationsaktivitäten potenzieller Bewerber zu finden sind, liegen. Diese Frage ist nicht nur aus der Bewerberperspektive von Interesse, sondern spielt auch für die Unternehmen als Arbeitgeber eine gewichtige Rolle. Neben den Bewerberwartungen wollen die Unternehmen auch die Kosten einer Bewerbungsentscheidung besser einschätzen können.

Ein wesentlicher Grund für die hohe Verbreitung der VIE-Theorie ungeachtet ihrer vorhandenen Defizite dürfte die explizite Forschungsmethodik sein, die VROOM (1966) mit seiner Theorie vorgelegt hat. Zugleich wird in der Literatur vermehrt auf die Notwendigkeit einer empirischen Überprüfung und inhaltlichen Weiterentwicklung der oben dargestellten Kernannahmen hingewiesen (VAN EERDE und THIERRY, 1996). 


\subsection{Determinanten der Arbeitgeberwahl aus prozessualer Sicht}

In ihrem Prozessmodell postulieren MOSER und ZEMPEL (2006), dass das Personalmarketing von Unternehmen wesentlich durch die zugrunde liegenden Entscheidungen potenzieller Bewerber beeinflusst wird. Dementsprechend erscheint es angemessen, auch die Arbeitgeberwahl aus der Perspektive der potenziellen Bewerber prozessual zu betrachten. Allerdings können die bisher vorgestellten theoretischen Ansätze diesen Beitrag nicht leisten: Während die erwartungstheoretischen Ansätze bei der Alternativenbewertung und -auswahl von einer einmaligen Entscheidungssituation ausgehen, zu der sämtliche Informationen verfügbar sind, fokussieren die informationsökonomischen Ansätze auf das Informationsverhalten, ohne dabei das dazugehörige Entscheidungsverhalten näher zu beschreiben. Daher werden im Folgenden Modelle vorgestellt, die die gesamte Arbeitgeberwahl in mehrere (Entscheidungs-)Phasen gliedern und damit ihren prozessualen Charakter hervorheben.

\subsubsection{Prozessmodell von Soelberg}

Im Kontext der Arbeitgeberwahl nimmt das von SOELBERG (1967, zit. in POWER und ALDAG, 1985) konzipierte Prozessmodell eine bedeutende Stellung ein. Dieses Prozessmodell beruht auf dem ,generalizable decision processing model“", das zur Erklärung und Verbesserung des Problemlösungsprozesses in wichtigen und einmaligen Entscheidungssituationen entwickelt wurde. Obwohl das Modell in seiner theoretischen Konzeption keinen situationsspezifischen Fokus aufweist, wurde es aufgrund der dazugehörigen empirischen Studie an MBA-Studenten ausschließlich im Kontext der Arbeitgeberwahl angewendet (OSBORN, 1990). Demnach lässt sich die Arbeitgeberwahl als ein mehrstufiger Prozess auffassen, der aus insgesamt vier Phasen besteht:

1. Entwicklung von Vorstellungen über einen Idealberuf

2. Planung der Arbeitsplatzsuche

3. Suche und Wahl eines Arbeitsplatzes'

4. Entscheidungsbestätigung und Entwicklung von Commitment

Die erste Phase ist dadurch gekennzeichnet, dass der potenzielle Bewerber seinen idealen Beruf festlegt. Aus dem Durchlaufen dieser Phase resultieren drei Typen von Bewerbern, die sich darin unterscheiden, ob sie keine, eine einzige oder mehrere Ideallösungen aufweisen. An die erste Phase schließen sich die Aktivitäten der zweiten Phase an, in der zwecks Arbeitsplatzsuche drei voneinander abhängige Aufgaben zu lösen sind (POWER und ALDAG, 1985). Neben der Entwicklung von Kriterien, anhand derer der zukünftige Arbeitgeber beurteilt werden kann, erfordert die Arbeitsplatzsuche zusätzlich Aufmerksamkeit, Zeit, Geld sowie bestimmte vom potenziellen Bewerber zu erbringende Anstrengungen. Schließlich gilt es, geeignete und effi- 
ziente Bewerbungswege zu identifizieren. SOELBERG (1967, zit. in POWER und ALDAG, 1985) unterscheidet hierzu vier verschiedene Strategien:

- Auf der Grundlage einer präzisen Vorstellung von seinem Idealberuf sucht der potenzielle Bewerber nach solchen Einstiegspositionen, in denen der Idealzustand erreicht werden kann.

- Die Arbeitsplatzsuche vollzieht sich über die üblichen Berufs- und Karrierepfade, die den Bewerber zum angestrebten Beruf führen.

- Der Bewerber orientiert sich an spezifischen Merkmalen des Idealberufs, die zwar bereits beim ersten Arbeitsplatz vorliegen sollten, jedoch ohne dass sie zugleich in eine weiterführende Karriereplanung eingebunden sind.

- Die Arbeitsplatzsuche ist flexibel angelegt, sodass Arbeitsplatzalternativen mit möglichst vielen Optionen vorliegen, die eine gute Ausgangsbasis für diverse Karrierewege offenhalten.

In der dritten Phase findet die eigentliche Arbeitgeberwahl statt. Hierbei werden die vorhandenen Arbeitsplatzalternativen im Hinblick auf die zuvor definierten Kriterien überprüft, d.h. sie werden akzeptiert oder verworfen, wobei das Modell sich auf Bedingungen konzentriert, unter denen es zu einer Änderung der Kriterien kommt. Als wesentlich werden vier Faktoren betrachtet: eine zu hohe Alternativenanzahl, die nicht mittels der vorhandenen Kriterien reduziert werden kann, ein beschränkter Umfang der zur Suche notwendigen Ressourcen, eine vorausgehende Absage für einen angestrebten Arbeitsplatz und schließlich die Identifikation eines erwünschten Arbeitsplatzes.

Infolgedessen postuliert SoelBerg (1967, zit. in POWER und AlDAG, 1985), dass das Ergebnis dieser Phase häufig in einer impliziten Arbeitgeberwahl zu sehen ist. Der zuvor ablaufende Informationsprozess ist vor allem dadurch bestimmt, dass Bewerber verschiedene Alternativen mit ihren Minimalanforderungen, die beispielsweise in der Gehaltshöhe liegen könnten, abgleichen. Die eigentliche Arbeitsplatzsuche gilt dann als beendet, wenn die bevorzugte Alternative mit einer hohen Wahrscheinlichkeit einhergeht, dort einen Arbeitsplatz angeboten zu bekommen. Andernfalls wird der Suchvorgang solange fortgesetzt, bis dem Bewerber mindestens zwei akzeptable Arbeitsplatzalternativen vorliegen, aus denen er anschließend seine Wahl treffen kann.

Das Ziel der vierten Phase besteht schließlich darin, Bestätigung und soziale Unterstützung für die implizit getroffene Wahl zu finden. Das in dieser Phase ebenfalls zu beobachtende Informationsverhalten der Bewerber dient im Gegensatz zu den vorausgegangenen Stufen primär der 
Rechtfertigung der getroffenen Wahl, wozu insbesondere rationale Gründe angeführt werden. Wenn abschließend sichergestellt ist, dass der Bewerber keine bessere Arbeitsplatzalternative findet, wird seine Entscheidung bekannt gegeben (POWER und ALDAG, 1985).

Soelberg (1967, zit. in Power und AldAG, 1985) geht in seinem Modell davon aus, dass Bewerber im Rahmen der Arbeitgeberwahl sowohl simultane als auch sequenzielle Entscheidungsstrategien verfolgen, wobei letzteren die Überlegung zugrunde liegt, dass ein Arbeitsplatzangebot als akzeptabel eingeschätzt und die Arbeitsplatzsuche abgebrochen wird, sobald individuelle Minimalstandards überschritten werden . Diese Grundannahme konnte in einer Studie von GLUECK (1974, zit. in POWER und ALDAG, 1985), die eine der wenigen empirischen Überprüfungen des Modells darstellt, prinzipiell bestätigt werden. Dabei zeigte sich am Beispiel von Hochschulabsolventen, dass potenzielle Bewerber drei verschiedene Entscheidungsmuster verwenden: Während bei der Maximierungsstrategie möglichst viele Alternativen innerhalb eines bestimmten Zeitraumes gefunden und zur finalen Auswahl herangezogen werden sollen, besteht die Validierungsstrategie aus dem Vergleich von insgesamt zwei Stellenangeboten, die nach einem uneingeschränkten Zeitintervall vorliegen müssen. Demgegenüber ist das Ziel der Zufriedenstellungsstrategie erreicht, sobald ein halbwegs annehmbares Angebot vorliegt. Aus der Gegenüberstellung der drei Strategien wird deutlich, dass die ersten beiden nach dem Prinzip der simultanen Abwägung verfahren, wohingegen die dritte Strategie auf dem Prinzip der minimalen Standards basiert (NERDINGER, 1994). Allerdings weist die Studie POWER und ALDAG (1985) zufolge einige methodische Mängel auf, sodass die skizzierte Befundlage weiteren empirischen Prüfungen zu unterziehen wäre. Auch andere direkte Überprüfungen des Prozessmodells sehen die Autoren mit zahlreichen methodischen Problemen verbunden, wie beispielsweise der Abgrenzung einzelner Phasen oder der Messung der Unsicherheit in der vierten Phase, behaftet und somit nicht als empirische Modellbestätigung zu werten. Ebenso liegt bislang kein fundierter Beleg für die Modellannahme vor, dass jeder Bewerber sämtliche Phasen in der vorgegebenen Reihenfolge durchläuft. Überdies geht aus dem Modell nicht hervor, anhand welcher Arbeitsplatzmerkmale potenzielle Bewerber ihre Arbeitgeber bewerten. Schließlich erscheint es fraglich, ob potenzielle Bewerber tatsächlich bereits in der ersten Phase über eine genaue Vorstellung ihres Idealberufes verfügen (NERDINGER, 1994).

Der hohe Stellenwert dieses Prozessmodells lässt sich vor allem dadurch erklären, dass es dem gesamten Prozess der Arbeitgeberwahl ein theoretisches Rahmenmodell zugrunde legt, wodurch diverse Untersuchungsansätze und Ergebnisse strukturiert werden können. Als kritisch erweist sich dabei allerdings, dass das Modell in seiner gesamten Konzeption auf die kognitiven Prozesse fokussiert, wobei die konativ entscheidenden Vorgänge, nämlich die Suche und Auswahl eines Arbeitsplatzes, lediglich in einer einzigen Phase verdichtet werden (NERDINGER, 1994). 
Die theoretischen Ansätze des oben dargestellten Prozessmodells finden sich in späteren Forschungsarbeiten wieder. So postuliert TEUFER (1999) ein zweistufiges Modell der Arbeitgeberwahl, das sich aus einer Selbstselektionsphase und einer darauf folgenden Bewertungs- und Entscheidungsphase zusammensetzt. Jede Phase ist durch unterschiedliche Arbeitsplatzmerkmale gekennzeichnet: Während in der Selbstselektionsphase die Branchen-, Unternehmensund Standortimages verankert sind, besteht die Bewertungs- und Entscheidungsphase aus den personalpolitischen Parametern und dem sog. „feel-good“ Faktor. Die Entscheidung für einen Arbeitgeber trifft der potenzielle Bewerber schließlich aufgrund des daraus resultierenden Arbeitgeberimages. Sein Modell kann jedoch aufgrund mehrerer Gesichtspunkte nicht überzeugen. Zum einen fehlt dieser inhaltlichen Aufteilung nach Images eine theoretisch fundierte Grundlage, sodass die bei den unterschiedlichen Prozessschritten Fragen nach der Vollständigkeit und Überscheidungsfreiheit bestehen. Darüber hinaus konnte die phasenspezifische Relevanz bestimmter Arbeitsplatzmerkmale nicht hinreichend bestätigt werden.

Das Modell von SIMON et al. (1995) legt der Arbeitgeberwahl einen vierstufigen Prozess zugrunde. In den ersten beiden Phasen wird die Bewerbungsentscheidung durch die Bekanntheit und Attraktivität des potenziellen Arbeitgebers beeinflusst. In der dritten Phase wird indes geprüft, ob der potenzielle Bewerber seine Entscheidung ernsthaft beabsichtigt hat. In der abschließenden Phase wird aus mehreren Arbeitsplatzangeboten das am meisten präferierte Stellenangebot ausgewählt. Obgleich Attraktivität und Präferenz in diesem Modell als die beiden zentralen psychologischen Konstrukte postuliert werden, fehlt darin eine fundierte theoriegeleitete Basis, sodass der hiermit erbrachte Erklärungsbeitrag zumindest bislang als begrenzt zu werten ist.

\subsubsection{Prozessmodell von Süß}

Zur Erklärung der im Rahmen der Arbeitgeberwahl stattfindenden Entscheidungsprozesse werden in der marketingwissenschaftlichen Literatur häufig Erkenntnisse aus der Konsumentenverhaltensforschung herangezogen (BÖTTGER, 2012). Die Konsumentenverhaltensforschung befasst sich vorrangig mit der Fragestellung, welche internen und externen Faktoren die Kaufentscheidung eines Konsumenten beeinflussen (TROMMSDORFF, 2011). Die dabei gewonnenen Erkenntnisse bzw. Typologien werden unter Berücksichtigung der zentralen theoretischen Konstrukte auf den Prozess der Arbeitgeberwahl übertragen. Demzufolge wird die Arbeitgeberwahl in der bisherigen Forschung als eine extensive Kaufentscheidung eingeordnet, da der zugrundeliegende Entscheidungsprozess alle hierfür relevanten Kriterien erfüllt: Die Entscheidung für einen Arbeitgeber ist ein längerfristig bindender, mit weitreichenden Konsequenzen einhergehender und komplizierter Prozess, der mit einem hohen Informationsbedürfnis verbunden ist und bei dem verschiedene Alternativen anhand eigens aufgestellter Bewertungskriterien abgewogen werden (TEUFER, 1999). 
Das Modell der Arbeitgeberwahl von SÜSS (1996) ist theoretisch eng an die Erkenntnisse aus der Konsumentenverhaltensforschung angelehnt. Infolgedessen ist sein dreistufiges Modell insbesondere dadurch gekennzeichnet, dass unterschiedliche Entscheidungsphasen mit einem unterschiedlichen Grad an Involvement und dem daraus resultierenden Informationsverhalten von Bewerbern einhergehen. Die zugrunde liegenden Phasen umfassen folgende Informationsbzw. Entscheidungsaspekte:

1. Noch bevor ein potenzieller Bewerber an konkreten Arbeitgeberinformationen interessiert ist, bilden sich bei ihm Unternehmensimages heraus, die vorrangig auf unpersönliche und indirekte Kommunikationsmedien zurückzuführen sind. Diese Phase wird aufgrund des niedrigen Involvements als „Low Involvement-Phase“ bezeichnet.

2. In der „Präferenzbildungs-Phase“ nehmen das Interesse an künftigen Arbeitgebern und das damit einhergehende Involvement deutlich zu, wodurch das Informationsbedürfnis des potenziellen Bewerbers steigt. Hieraus resultieren persönliche Einstellungen gegenüber möglichen Arbeitgebern. Am Ende dieser Phase entwickeln sich eindeutige Bewerberpräferenzen, die eine begrenzte Anzahl an Alternativen umfassen.

3. Die „Critical Contact-Phase“ beinhaltet neben der Bewerbungsentscheidung den direkten Kontakt mit den in Frage kommenden Arbeitgebern, die Teilnahme an Auswahlverfahren sowie schließlich auch die Annahme eines Arbeitsplatzangebotes. Aufgrund des sehr hoch ausgeprägten Involvements aufseiten des potenziellen Bewerbers sind die hierbei gewonnen Eindrücke als entscheidungsrelevant für seinen Organisationseintritt zu werten.

Die Stärke des Modells von SÜSS (1996) liegt primär in seiner praxisorientierten Anwendung. So lassen sich aus dem phasenspezifischen Involvement auch die bevorzugten Kommunikationsmedien potenzieller Bewerber ableiten. Die wichtigsten Kritikpunkte an der Konzeption dieses Modells lassen sich indes wie folgt zusammenfassen (vgl. MALMENDIER, 2006). Zum einen werden die zentralen Modellkonstrukte wie Involvement und die phasenspezifischen Aktivitäten des potenziellen Bewerbers ausschließlich durch die einzelnen Entscheidungsphasen determiniert. Dabei wird allerdings nicht auf die zugrunde liegenden Wirkungsbeziehungen zwischen den einzelnen Konstrukten wie Arbeitgeberimage, Involvement und Informationsverhalten eingegangen. Darüber hinaus werden die Modellannahmen empirisch nicht überprüft, sodass die einzelnen Phasen und die darin postulierten Entscheidungsvorgänge bislang nur einen theoretischen Charakter haben. Schließlich wird anhand des Prozessmodells nicht aufgezeigt, wie die Entscheidungen potenzieller Bewerber in den einzelnen Phasen getroffen werden. 


\subsection{Zwischenfazit}

Die oben dargestellten Ansätze zur Erklärung der Arbeitgeberwahl führen aufgrund mehrerer gewichtiger Gründe bislang nicht zu zufriedenstellenden Antworten. So geben sie zum einen wenig Aufschluss darüber, wie das Entscheidungsverhalten potenzieller Bewerber gestaltet ist. Dieser Einwand betrifft beispielsweise die Annahme, dass unterschiedliche Entscheidungsmodelle je nach Bewerbungs- bzw. Rekrutierungsphase relevant sein könnten. Während erwartungstheoretische Modelle letztlich postulieren, dass verschiedene wahrgenommene Arbeitsplatzmerkmale in kompensatorischer Weise beurteilt werden, setzen sequenzielle Entscheidungsmodelle das Überschreiten eines Mindestwertes bei den Ausprägungen einzelner Arbeitsplatzmerkmale voraus. Die an erwerbslosen Personen durchgeführten Untersuchungen konnten zeigen, dass viele potenzielle Bewerber bei direkter Befragung über minimale Standards hinsichtlich des angestrebten Gehalts verfügen (vgl. zusammenfassend: SCHWAB et al., 1987). Jedoch ist die Arbeitsmarktsituation der zugrundeliegenden Stichprobe nicht mit der von Studierenden und Hochschulabsolventen vergleichbar, sodass nach dem bisherigen Forschungsstand eher von einem simultanen Entscheidungsmodell ausgegangen werden kann (NERDINGER, 1994). Allerdings wird auch das simultane Entscheidungsmodell, das auf der kompensatorischen Bewertung einzelner Arbeitsplatzmerkmale basiert, aufgrund von methodischen Einschränkungen der entsprechenden empirischen Studien kritisch betrachtet.

Darüber hinaus wird die Bewerbungsentscheidung sowohl in den Job-Search-Modellen als auch in den prozessorientierten Modellen lediglich als Teil der Alternativensuche gesehen (vgl. MALMENDIER, 2006). Gleichzeitig heben aber zahlreiche empirische Studien die Bedeutung der Bewerbung als eigenständige Entscheidung hervor (vgl. für einen Überblick: CHAPMAN et al., 2005). Hierbei wird davon ausgegangen, dass die relativ früh im Prozess der Arbeitgeberwahl zu fällende Bewerbungsentscheidung insbesondere im Image von Unternehmen begründet liegt, das seinerseits die Arbeitgeberattraktivität beeinflusst (EHRHART und ZIEGERT, 2005). Ein wesentliches Defizit der entsprechenden Ansätze ist allerdings darin zu sehen, dass sie bislang nicht alle relevanten Konstrukte berücksichtigen (BOSWELL et al., 2012). So wird in einigen marketingwissenschaftlichen Studien lediglich die Einstellungskomponente in Form von Arbeitgeberattraktivität zur Vorhersage der Bewerbungsabsicht erfasst (vgl. z.B. KIRCHGEORG und GÜNTHER, 2006; HAUTZINGER, 2009; BÖTTGER, 2012). Bei diesem kausalen Zusammenhang zwischen Einstellung und Verhalten wird jedoch die Erkenntnis vernachlässigt, dass das Verhalten bzw. die Verhaltensintention auch von anderen Faktoren beeinflusst wird, von denen Einstellungen nur eine Klasse darstellen (EAGLY und CHAIKEN, 1993). Diese Erkenntnis hat dazu geführt, dass in den meisten gegenwärtig diskutierten Theorien zur Einstellungs-Verhaltens-Beziehung weitere Determinanten des individuellen Verhaltens einbezogen werden, die in den Modellen zur Arbeitgeberwahl bislang zu wenig Berücksichtigung finden. 


\subsection{Das Modell der Person-Organisation-Passung}

Als (Fremd-)Selektion wird in der organisationspsychologischen Literatur überwiegend die Auswahl von Bewerbern bezeichnet, die für eine betriebliche Position geeignet sind (NERDINGER, 1994). Zugleich verdeutlicht diese Definition die weit verbreitete Perspektive, der zufolge die Personalauswahl einen einseitigen Prozess darstellt, in dem sich Organisationen mit dem erforderlichen Personal versorgen. Diese Sichtweise geht dabei von einer Arbeitsmarktsituation aus, in der das Produkt Arbeitsplatz auf eine ausreichende oder gar übermäßige Nachfrage trifft (MOSER und ZEMPEL, 2006). Betrachtet man allerdings die im Abschnitt 2.4 dargestellten Szenarien zur mittelfristigen Entwicklung auf dem Arbeitsmarkt, so haben sich viele Unternehmen mittel- und langfristig auf einen spürbaren Fach- und Führungskräftemangel einzustellen (KAY et al., 2010). Die prognostizierte Arbeitsmarktsituation veranlasst zugleich dazu, den klassischen Blick auf die Selektion umzukehren und stattdessen zu fragen, wie ein potenzieller Bewerber seinen zukünftigen Arbeitgeber auswählt (KIRCHGEORG und MÜLLER, 2011). Die von potenziellen Bewerbern ausgehenden Aktivitäten und Entscheidungen werden in der Psychologie als Selbstselektion bezeichnet. Solche Selektionseffekte stehen im Mittelpunkt des attraction-selection-attrition-Modells (ASA-Modell) von SCHNEIDER (1987), das sich aus insgesamt drei Teilprozessen zusammensetzt. Eine zentrale Modellannahme besteht hierbei darin, dass die Organisationsmitglieder eine relativ homogene Persönlichkeit aufweisen. Diese Homogenität wird dadurch erklärt, dass diese Personen sich bei der gleichen Organisation beworben haben, von ihr aus einem Bewerberpool als geeignet eingestuft und ausgewählt wurden und sich schließlich dazu entschlossen haben, in der Organisation zu verbleiben (SCHNEIDER et al., 1995). Die Selbstselektion kommt gemäß diesem Modell dadurch zustande, dass die Organisationsstruktur, -kultur und -strategie eine differenzielle Anziehung auf potenzielle Bewerber ausüben (ROSENSTIEL und NERDINGER, 2000). Dieser Teilprozess wird innerhalb des Modells entsprechend als attraction bezeichnet. Unter selection werden hingegen die zwei ineinander greifenden Prozesse der Selbst- und Fremdselektion verstanden. Demzufolge werden bei der Bewerbungsentscheidung solche Unternehmen bevorzugt, von denen angenommen wird, dass die eigenen Werthaltungen, selbst gesetzten Ziele und Anforderungen dort realisiert werden können. Hieraus resultiert ein relativ homogener Bewerberpool, aus dem Organisationen durch formale und informale Fremdselektion diejenigen auswählen, die in ihren Kompetenzen, Persönlichkeitsmerkmalen, Interessen und Werthaltungen am besten zur Organisation passen. Folglich wirkt die Fremdselektion verstärkend auf die Homogenitätsbildung hinsichtlich der Persönlichkeit der Organisationsmitglieder. Schließlich stabilisiert sich dieser Homogenisierungsprozess, da Personen, die in ihrer Persönlichkeit und ihren Werthaltungen nicht zu einer Organisation passen, diese eher verlassen bzw. verlassen müssen (ROSENSTIEL und NERDINGER, 2000). Das Verlassen einer Organisation stellt seinerseits den letzten, optionalen Teilprozess innerhalb des ASA-Modells dar und wird als attrition bezeichnet. Sämtliche Teilprozesse des ASA-Modells wurden empirisch bestätigt (DE COOMAN et al., 2009). So wurde beispielsweise in einer groß- 
angelegten Untersuchung gezeigt, dass sich die Führungskräfte unterschiedlicher Organisationen auch in ihrer Persönlichkeitsstruktur unterscheiden lassen (SCHNEIDER et al., 1998).

Das ASA-Modell verdeutlicht mit seinen Kernannahmen den Stellenwert, der sich aus der Passung der Person mit der entsprechenden Organisationskultur ergibt. Dabei postuliert es, dass die Arbeitgeberwahl vor dem Hintergrund der eigenen Persönlichkeitsmerkmale, Ambitionen und Werthaltungen erfolgt (SCHNEIDER et al., 1998). Eine psychologische Konzeption, die sich gezielt mit diesem Passungsprinzip befasst, stellt das Rahmenmodell zur Person-Umwelt-Passung von KRISTOF (1996) dar. Hierin wird es als ,the compatibility between people and organizations that occurs when: (a) at least one entity provides what the other needs, or (b) they share similar fundamental characteristics, or (c) both" definiert (KRISTOF, 1996: 4 f.). Die Anpassung von Mitarbeitern kann zugleich in unterschiedlichen Umwelten erfolgen, worunter die Übereinstimmungen mit einem bestimmten Beruf (person-vocation fit), mit einer bestimmten Organisation (person-organisation fit), mit einem bestimmten Anforderungs- bzw. Aufgabenbereich (person-job fit) sowie mit einer bestimmten Arbeitsgruppe (person-group fit) fallen. Allen vier Perspektiven ist die Sichtweise gemeinsam, dass die Passung dann am besten gelingt, wenn die Umweltbedingungen mit den eigenen Persönlichkeitsmerkmalen und Werthaltungen möglichst gut übereinstimmen.

In den bisherigen Untersuchungen hat das Modell der Person-Organisation-Passung die größte Aufmerksamkeit unter den vier Modellen zur Person-Umwelt-Passung erfahren, wodurch hierfür auch die meisten theoretischen Ausdifferenzierungen und empirischen Befunde zu finden sind (HOFFMAN und WOEHR, 2006). Hierbei wird angenommen, dass die subjektive Passung zwischen Person und Organisation aus mehreren Dimensionen besteht (KRISTOF, 1996). So kann die Wahrnehmung eines Unternehmens zum einen entlang der Demands-Abilities Dimension erfolgen, in der die Übereinstimmung organisationaler Anforderungen mit dem Wissen und den Kompetenzen der Person ausschlaggebend ist. Davon ist die Needs-Supplies Dimension abzugrenzen, der zufolge Personen nach solchen Organisationen und den damit einhergehenden Arbeitsplatzmerkmalen suchen, in denen sie ihren individuellen Bedürfnissen nachkommen können. Diesen beiden Dimensionen liegt das komplementäre Passungsprinzip zugrunde, demzufolge die persönlichen und organisationalen Merkmale einander ergänzen. Demgegenüber entscheiden sich potenzielle Bewerber gemäß dem supplementären Passungsprinzip eher für solche Organisationen, deren wahrgenommene Merkmale, gebündelt im Arbeitgeberimage, ihrem eigenen Selbstbild am ähnlichsten sind. Das Arbeitgeberimage setzt sich dabei aus subjektiven Meinungen, Überzeugungen und Gefühlen gegenüber der Organisation zusammen (BARBER, 1998). 


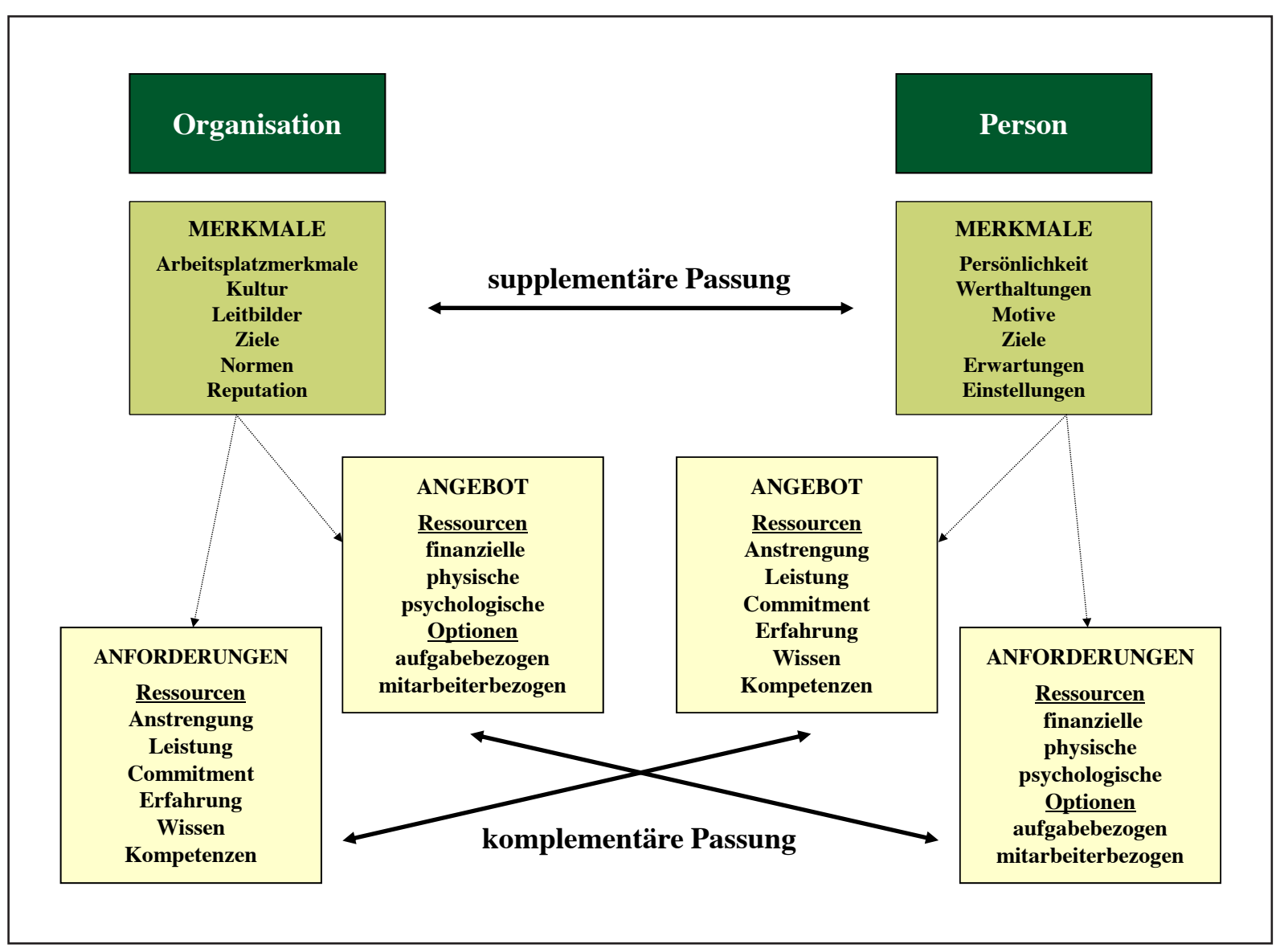

Abb. 3-1: Modell der Person-Organisation-Passung

(Eigene Darstellung, 2011, in Anlehnung an KRISTOF, 1996)

Für eine fundierte Betrachtung der Arbeitgeberwahl ist das Konzept der Person-Umwelt-Passung aus mehreren Gründen nützlich. Der Stellenwert dieses Ansatzes ist zum einen in der Berücksichtigung des Selbstkonzeptes zu sehen. So weisen marketingwissenschaftliche Studien darauf, dass die explizite Berücksichtigung unterschiedlicher Bewerbersegmente sehr bedeutsam ist (MEFFERT et al., 2012). Auch die psychologischen Studien zur Arbeitgeberwahl beziehen zunehmend interindividuelle Unterschiede in ihre Forschungsfragen mit ein (vgl. z.B. LIEVENS et al., 2001; RESICK et al., 2007; SCHREURS et al., 2009). Die zentrale Annahme besteht hierbei darin, dass unterschiedliche Bewerbererwartungen, die im jeweiligen Selbstkonzept begründet liegen, vor allem für die anfängliche Attraktion von Bedeutung sind. Das Modell der Person-Umwelt-Passung stellt an dieser Stelle einen angemessenen heuristischen Rahmen dar, um diese interindividuellen Unterschiede theoretisch zu verankern. 
Ferner ist aufgrund der Explikation unterschiedlicher Passungsmodelle eine differenzierte Analyse der Arbeitgeberwahlentscheidung möglich. Wird die Arbeitgeberwahl als eine Folge von Bewerberentscheidungen aufgefasst, so ist es annehmbar, dass in unterschiedlichen Bewerbungs- bzw. Rekrutierungsphasen andere Umwelten und somit Passungsmodelle entscheidungsrelevant sind. Zur Überprüfung dieser Annahme finden sich in der Literatur bereits einige Hinweise, denen zufolge die Person-Organisation-Passung eher die Attraktivitätseinschätzung eines Arbeitgebers beeinflusst, während die Person-Job-Passung die prozessual betrachtet später erfolgende Entscheidung für die Arbeitsplatzannahme beeinflusst (vgl. DINEEN et al., 2002; CARLESS, 2005; CHAPMAN et al., 2005). Hieraus lässt sich zugleich schlussfolgern, dass vor allem die anfängliche Bewerbungsentscheidung von der Kongruenz zwischen den persönlichkeitsspezifischen Merkmalen potenzieller Bewerber und dem jeweiligen Arbeitgeberimage abhängig ist. Entscheiden sich die potenziellen Bewerber für eine weiterführende Teilnahme am Rekrutierungsprozess, dann kann eher die Person-Job-Passung zur Tragen kommen.

\subsection{Image-Theorie}

Um die Relevanz persönlichkeitsspezifischer Merkmale bei der Bedeutsamkeitseinschätzung von Arbeitsplatzmerkmalen theoretisch zu untermauern, werden die Annahmen der Image-Theorie von BEACH (1990) herangezogen. Die Image-Theorie geht von drei entscheidungsrelevanten Wissensstrukturen, sog. Images aus, worunter das Value Image, das Trajectory Image und das Strategic Image fallen. Die Images werden als ,schemata that are specific to decision behavior and represent the decision maker's guiding principles relevant to some sphere of decision making” definiert (BEACH und MiTCHELL, 1987: 202). Das Value Image besteht überwiegend aus persönlichen Wertvorstellungen. Hierzu zählen aber auch internalisierte soziale Konventionen, die im alltäglichen Leben die Entscheidungssituationen dominieren. Die persönlich bedeutsamen Ziele, die beispielsweise aus den Wertvorstellungen bzw. Prinzipien des Value Image abgeleitet wurden, bilden hingegen das Trajectory Image. Hierin werden folglich alle Ziele repräsentiert, die eine Person erreichen will. Zugleich kann der Abstraktionsgrad von Zielen variieren, sodass sowohl konkrete als auch übergeordnete Ziele in dieser Struktur enthalten sind. Schließlich umfasst das Strategic Image Handlungspläne und Umsetzungsstrategien zur Verwirklichung von persönlichen Zielen. Die einzelnen Handlungsschritte, die innerhalb der Zielumsetzung erfolgen, werden als tactics bezeichnet. BEACH (1990) postuliert ferner, dass Handlungspläne zum einen eine Steuerungsfunktion innehaben und zum anderen die Antizipation von Handlungsergebnissen ermöglichen. Der Entscheidungsprozess wird insofern kontinuierlich (neu-)bewertet, als dass man die antizipierten Handlungsfolgen den persönlichen Zielvorstellungen aus dem Trajectory Image gegenüberstellt und eine entsprechende Bilanz zieht. Diese theoretische Konzeption weist ihrerseits Ähnlichkeiten zu dem Means-End-ChainAnsatz auf, der auf der Annahme basiert, dass Wissen und Erfahrung durch die kognitiven 
Kategorien Attribute, Konsequenzen und Werte hierarchisch im Gedächtnis organisiert sind (BALDERJAHN und WIEDEMANN, 1999).

Die oben dargestellte Analyse der zugrundeliegenden kognitiven Entscheidungsstruktur wird in der Image-Theorie um ein Phasenmodell ergänzt, das den prozessualen Entscheidungscharakter deskriptiv abbildet. BEACH und MITCHELL (1987) gehen hierbei von zwei grundsätzlich unterschiedlichen Entscheidungstypen aus: Während die sog. Adoption-Entscheidungen auf die Akzeptanz bzw. Zurückweisung kognitiver Inhalte vor dem Hintergrund der drei Images fokussieren und somit eine Zielbildungsfunktion beinhalten, gehen Progress-Entscheidungen mit der Frage einher, ob die erfolgte Zielimplementierung und -umsetzung im Hinblick auf das Strategic Image als zufriedenstellend zu werten ist. Aus der zeitlichen Perspektive sind die Progress-Entscheidungen den Adoption-Entscheidungen nachgelagert. Die Adoption-Entscheidungen werden ihrerseits in zwei weitere Entscheidungsphasen untergliedert: Screening und Choice (BEACH, 1990). Die Screening-Phase umfasst dabei die Prüfung der verfügbaren Optionen im Hinblick darauf, ob sie die Bestandteile der drei Images verletzen. Diese Entscheidungsstrategie wird als Kompatibilitätstest bezeichnet. Demzufolge wird innerhalb dieser Phase für jede einzelne Wahlalternative gesondert geprüft, ob diese grundsätzlich mit den drei individuellen Images vereinbar bzw. kompatibel ist. Der Kompatibilitätstest fokussiert nur auf Verletzungen von Standards (violations) und ist somit nicht kompensatorisch (vgl. JUNGERMANN et al., 2010). Dabei wird eine Option unwiderruflich zurückgewiesen, wenn die gewichtete Summe von Standardverletzungen eine bestimmte Schwelle, die als rejection threshold bezeichnet wird, überschreitet. Da der zugrundeliegende Entscheidungsmechanismus sich nur auf Verletzungen von Prinzipien bezieht und somit asymmetrisch und nicht kompensatorisch ist, kann diese Unvereinbarkeit zugleich nicht durch eine adäquate Übereinstimmung mit anderen Standards ausgeglichen werden. Wenn in der Screening-Phase alle verfügbaren Optionen gegen Standards verstoßen und daraufhin zurückgewiesen werden, so kann keine Entscheidung getroffen werden. In diesem Fall muss die Entscheidungssituation verlassen oder neu definiert werden. Innerhalb der Adoption-Entscheidungen besteht die zentrale Aufgabe des Kompatibilitätstests somit darin, die mit den drei oben aufgeführten Images inkompatiblen Ziele und Handlungsalternativen zu identifizieren und zu entfernen (BEACH, 1990; ORDOÑEZ et al., 1999). Des Weiteren nehmen BEACH und MitCHELL (1987) an, dass die multiattributive Bewertung innerhalb des Kompatibilitätstests teilweise auch implizit erfolgt. So ist das Objekt zwar beim Beurteiler kognitiv durch mehrere bewertungsrelevante Attribute repräsentiert, dennoch nimmt er häufig eine holistische bzw. intuitive Bewertung vor, indem er die Bewertungen des Objektes kognitiv ,irgendwie“ integriert (JUNGERMANN et al., 2010). Ein Bewerber könnte beispielsweise ein Stellenangebot als passend bewerten, ohne dass aus seiner Beurteilung hervorgeht, ob er das Stellenangebot auf allen Attributen bzw. Arbeitsplatzmerkmalen durchgängig als ,passend“ bewertet oder auf einigen als „sehr passend“, auf anderen hingegen als „weniger passend“ und erst insgesamt als ,passend“ betrachtet. Diese Annahme ist gleichzeitig konform mit den bishe- 
rigen Forschungsergebnissen zur Arbeitgeberwahl, denen zufolge die Wahrnehmung eines Arbeitgeberimages nicht unbedingt aus den additiv bewerteten Arbeitsplatzmerkmalen resultiert, sondern auf der Basis eines teilweise assoziativen Gesamteindrucks erfolgt (vgl. VON WALTER et al., 2009). Ähnlich verhält es sich mit der Unvereinbarkeit verfügbarer Optionen mit den eigenen Standards: Die Zurückweisung erfolgt hierbei nicht nur anhand einer bewussten Analyse der einzelnen Merkmale von Optionen, sondern größtenteils durch ein holistisches, intuitives Affekturteil (BEACH, 1990).

Den zweiten Schritt im Phasenmodell von BEACH (1990) stellt die Choice-Phase dar, in der mehrere Optionen, die den Prüfkriterien in der Screening-Phase gerecht wurden, einem sog. Profitabilitätstest unterzogen werden. Hierbei werden die Vor- und Nachteile einer Option auf kompensatorische Weise bewertet, was zur simultanen Auswahl der besten bzw. attraktivsten Option führt. Innerhalb dieser Konzeption können folglich unterschiedliche kompensatorische Entscheidungsregeln, wie beispielsweise das additive multiattributive Nutzen-Modell (MAURegel), zur Anwendung kommen. In diesem Zusammenhang wird angenommen, dass dem Profitabilitätstest im Gegensatz zum Kompatibilitätstest eine explizite Bewertung zugrunde liegt. Das bedeutet, dass der Beurteiler für das Objekt jeweils eine partielle, analytische Bewertung auf den verschiedenen Attributen vornimmt (JUNGERMANN et al., 2010). Die Aggregation dieser partiellen Nutzenbeurteilungen zu einem Gesamtwert hängt dabei von der Entscheidungssituation einerseits und der Strategie des Beurteilers andererseits ab.

Für die Analyse der Arbeitgeberwahl liefert die Image-Theorie einen vielversprechenden Ansatz, um subjektiv bedeutsame Zusammenhänge zwischen den Wertvorstellungen in Form von Berufsorientierungen potenzieller Bewerber und den Attributen eines Stellenangebotes zu identifizieren. Die Verwendung von Berufsorientierungen innerhalb des Value Image wird zugleich damit begründet, dass Werte als Konzeptionen des Wünschenswerten einen recht abstrakten Charakter aufweisen, während sich Berufsorientierungen als übergeordnete Zielintentionen auf explizite Bedingungen des Arbeitslebens beziehen und somit die konkrete berufliche Entscheidung beeinflussen (ROSENSTIEL und NERDINGER, 2000; MAIER et al., 2009). Demgegenüber entspricht das Trajectory Image den persönlichen beruflichen Zielen, worunter Absichten und Bestrebungen fallen, die eine Person in ihrer beruflichen Laufbahn realisieren möchte (BRUNSTEIN und MAIER, 1996). Im Vergleich zu den Wert- und Berufsorientierungen des Value Images, sind die persönlichen beruflichen Ziele sehr viel konkreter und fokussieren auf die kurz- bis mittelfristig zu erreichende Arbeitssituation, die im Unternehmen angestrebt wird (ROSENSTIEL und NERDINGER, 2000). Erst durch berufliche Ziele wird die grundsätzlich unendliche Menge an Optionen bzw. Arbeitgebern, Konsequenzen und Attributen bzw. Arbeitsplatzmerkmalen eingeschränkt, die der Bewerber in Betracht ziehen könnte (vgl. JUNGERMANN et al., 2010). So grenzt beispielsweise das Ziel, schnell eine hohe Position im Unternehmen zu erreichen, die in Betracht zu ziehenden Arbeitgeber (z.B. eher Großunternehmen) und als rele- 
vant erachteten Arbeitsplatzmerkmale (z.B. gute Entwicklungs- und Aufstiegschancen) ein. Zugleich wird der Einfluss von Wertvorstellungen bzw. Berufsorientierungen auf das individuelle Handeln über persönliche Ziele vermittelt (BROWN und CRACE, 1996). Schließlich können aus den persönlichen beruflichen Zielen konkrete Bewerbererwartungen abgeleitet werden, die ihrerseits unmittelbar die Bedeutsamkeitseinschätzung von Arbeitsplatzmerkmalen beeinflussen (vgl. BALDERJAHN und WiEDEMANN, 1999). Gemäß den Annahmen der Image-Theorie lassen sich die Bewerbererwartungen als fester Bestandteil des Strategic Image auffassen. Die hieraus resultierenden strukturellen Bestandteile aufseiten potenzieller Bewerber werden in Abbildung 3-2 veranschaulicht:

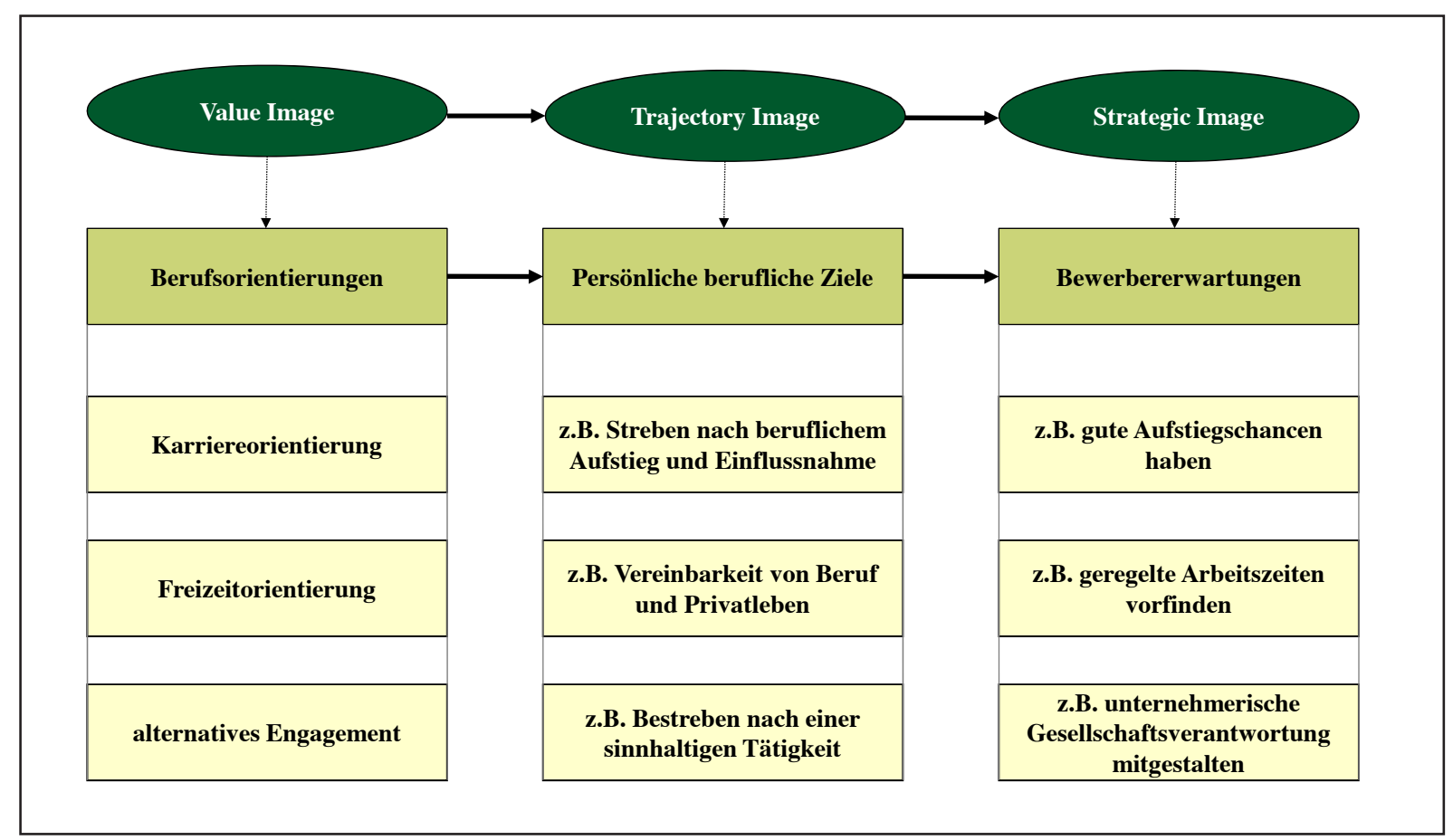

Abb. 3-2: Modell kognitiver Entscheidungsstrukturen eines potenziellen Bewerbers bei der Arbeitgeberwahl (Eigene Darstellung, 2011, in Anlehnung an BEACH, 1990; ROSENSTIEL und NERDINGER, 2000)

Abschließend hervorzuheben ist, dass sich die Image-Theorie auf extensive bzw. reflektierte Entscheidungen konzentriert, die eine hohe persönliche Relevanz aufweisen und somit eine bewusste Auseinandersetzung mit den eigenen Zielen und Erwartungen voraussetzen (JUNGERMANN et al., 2010). Auch dies unterstreicht den Stellenwert dieses theoretischen Ansatzes für die Analyse der Arbeitgeberwahl, die ihrerseits als eine extensive Entscheidung charakterisiert worden ist (TEUFER, 1999). 


\subsection{Theorie des geplanten Verhaltens}

Die Frage der Entscheidung für ein Unternehmen bzw. ein Stellenangebot bezieht sich auf die Verarbeitung von dazugehörigen Arbeitsplatzmerkmalen, die wiederum im Arbeitgeberimage eines Unternehmens gebündelt sind (LEMMINK et al., 2003; EHRHART und ZIEGERT, 2005). Generell liegen für das Imagekonstrukt unterschiedliche theoretische Ansätze vor, woraus auch abweichende Konstruktdefinitionen resultieren. Einer weit verbreiteten Definition von TROMMSDORFF (2009: 155) zufolge handelt es sich beim Produktimage um eine „,mehrdimensionale und ganzheitliche Grundlage der Einstellung einer Zielgruppe zum Gegenstand“. Diese Definition lässt sich in die einstellungspsychologisch orientierte Imagetheorie einordnen, die in der aktuellen Marketingforschung vorherrschend ist (vgl. ANDRATSCHKE et al., 2009). TEUFER (1999: 133) definiert das Arbeitgeberimage analog zum Produktimage als ,die Wahrnehmung eines Unternehmens bezogen auf die Ausprägungen derjenigen Merkmale, in denen sich seine Attraktivität als potentieller Arbeitgeber widerspiegelt"“. Gemäß der hier vorgelegten Definition bestimmt das Arbeitgeberimage die Einstellung, die potenzielle Bewerber einem Unternehmen als möglichem Arbeitgeber gegenüber entwickeln. Innerhalb dieser Sichtweise ist es somit sinnvoll, auf eine Theorie zurückzugreifen, die sich gezielt mit der Auswirkung von Einstellungen auf das Verhalten befasst. Eine solche Theorie stellt die Theorie des geplanten Verhaltens (theory of planned behavior, TOPB) von AJZEN $(1991,2008)$ dar, deren grundlegende theoretische Annahmen und entsprechende empirische Befunde im Folgenden dargestellt werden.

Für das Einstellungskonstrukt existieren zahlreiche Definitionsvorschläge, die jedoch häufig nur auf einen relevanten Teilaspekt fokussieren. Ein weitgehend konsensfähiger Definitionsvorschlag stammt von EAGLY und CHAIKEN (1993: 1), die das Einstellungskonstrukt folgendermaßen beschreiben: „Attitude is a psychological tendency that is expressed by evaluating a particular entity with some degree of favor or disfavor“. Den theoretisch zentralen Bestandteil dieser Definition stellt demzufolge eine Bewertung dar. Hierin werden zugleich alle Klassen von bewertenden Reaktionen subsumiert, unabhängig davon ob sie affektiver, kognitiver oder konativer Art sind und sich in ihrer Richtung und Intensität unterscheiden (SIX und ECKES, 1996). EAGLY und CHAIKEN (1993) gehen hierbei allerdings nicht von einer statischen Konsistenz der Affekte, Kognitionen und Intentionen aus, wie sie von einigen Forschern im Rahmen des sog. Dreikomponentenansatzes vertreten wird, sondern postulieren einen dynamischen Ausprägungscharakter der einzelnen Komponenten, der sich in Abhängigkeit des jeweiligen Einstellungsobjektes verändern kann. Der Begriff des Einstellungsobjektes wird ferner insofern präzisiert, als dass es in seinem Abstraktionsgrad variieren und sowohl auf bestimmte einzelne Verhaltensweisen als auch auf entsprechende Klassen von Verhaltensweisen gerichtet sein kann. 
Die Nützlichkeit des Einstellungskonstruktes in der sozialpsychologischen Forschung beruht weitgehend auf der Annahme, dass Einstellungen Verhalten beeinflussen (SIX und ECKES, 1996). Allerdings war gerade dieser Zusammenhang seit Anbeginn der Einstellungsforschung umstritten und wurde erst anhand mehrerer metaanalytischen Untersuchungen empirisch konsolidiert (JONAS und DOLL, 1996). Vor dem Hintergrund dieser metaanalytischen Ergebnisse stellen SIX und ECKES (1996) fest, dass das Einstellungskonstrukt der wichtigste, wenn auch nicht der alleinige Prädiktor für Verhalten ist. Die eingeschränkte prädiktive Validität des Einstellungskonstruktes führen die Autoren u.a. auf methodische Unzulänglichkeiten zurück, wozu sie beispielsweise eine unzureichende Operationalisierung, eine theoretisch wenig fundierte Betrachtung der Einstellungs-Verhaltens-Relation sowie eine weitgehende Vernachlässigung der Kompatibilität von Einstellungs- und Verhaltensmaßen zählen. Hinzu kommt, dass das Verhalten neben der Einstellungsdimension auf eine Reihe weiterer Einflussgrößen zurückzuführen ist (EAGLY und CHAIKEN, 1993). Aus dieser Erkenntnis heraus hat die Einstellungs-Verhaltens-Forschung zusätzliche Determinanten in die dazugehörigen Modelle integriert (JONAS und DOLL, 1996).

Die zentrale Grundannahme der TOPB besteht darin, dass ein eingeschränkt willentlich kontrolliertes Verhalten durch die Absicht und die subjektiv wahrgenommene Möglichkeit, dieses Verhalten auszuführen, determiniert wird (AJZEN, 1991). Die Intention bestimmt hierbei das Ausmaß der Anstrengung bzw. der Entschlossenheit, das die Person zur Verhaltensausführung aufbringen will. Dementsprechend impliziert diese Verhaltensdeterminante einen motivationalen Aspekt. Indes umfasst die wahrgenommene Verhaltenskontrolle die subjektive Einschätzung, wie einfach bzw. schwierig die Verhaltensausführung sein wird. Dieses Konstrukt fokussiert daher auf kontrollrelevante interne und externe Faktoren, wodurch der theoretische Geltungsbereich ausgehend von einer motivationalen Perspektive um die Berücksichtigung interner bzw. externer Ressourcen und Barrieren erweitert wird (JONAS und DOLL, 1996). Die Vorhersagekraft der wahrgenommenen Verhaltenskontrolle hängt AJZEN (1991) zufolge davon ab, inwieweit sie mit der tatsächlichen bzw. objektiven Verhaltenskontrolle übereinstimmt. Allerdings sind innerhalb dieses Zusammenhanges häufig Diskrepanzen zu beobachten. In der psychologischen Entscheidungsforschung wird dieses Phänomen dadurch erklärt, dass Personen ihre Urteile nur selten nach den Regeln der Wahrscheinlichkeitstheorie abgeben können, da ihnen die entsprechenden Informationen, Regeln oder Hilfsmittel zumeist nicht zur Verfügung stehen (JUNGERMANN et al., 2010). Sie treffen ihre Urteile folglich häufig intuitiv, wozu sie wiederum eher auf mentale Heuristiken, wie beispielsweise auf die Repräsentativitäts- oder Verfügbarkeitsheuristik, als auf Algorithmen der Wahrscheinlichkeitstheorie zurückgreifen. Allerdings führen solche Heuristiken zu subjektiven Urteilen, die nicht immer mit den objektiven bzw. regelbasierten Wahrscheinlichkeitsurteilen übereinstimmen. 
AJZEN (1991) formuliert mehrere Voraussetzungen, die erfüllt sein müssen, damit die Annahmen der TOPB zu einer zufriedenstellenden prognostischen Leistung führen. Erstens wird gemäß dem sog. Kompatibilitätsprinzip die prädiktive Validität der Verhaltensdeterminanten umso höher ausfallen, je mehr das Abstraktions- bzw. Spezifitätsniveau der Verhaltensdeterminanten mit dem zu erfassenden Verhalten korrespondiert (ECKES, 1996). So lassen sich zwischen den eher allgemein gehaltenen Einstellungen und eher spezifisch formulierten Verhaltensweisen überwiegend nicht signifikante Korrelationen beobachten, während aus einer hohen inhaltlichen Kompatibilität zwischen den Einstellungen und dem daraus vorherzusagenden Verhalten relativ hohe Korrelationen resultieren. Gleiches gilt AJZEN (1991) zufolge auch für den Intentions-Verhaltens-Zusammenhang, sodass die Vorhersage eines spezifischen Verhaltens auch eine spezifische Erfassung der zugrunde liegenden Intention erfordert.

Darüber hinaus ist die Güte der Prädiktoren von der zeitlichen und situativen Stabilität zwischen dem erfragten und dem beobachteten Verhalten abhängig. So wird hierbei postuliert, dass die Salienz von negativen Verhaltenskonsequenzen zunimmt, je näher der Zeitpunkt der Verhaltensausführung rückt, woraus wiederum eine veränderte Intention resultieren könnte (AJZEN, 1991). Die Wahrscheinlichkeit dafür, dass sich eine Intention verändert, wird dabei umso größer, je länger das zeitliche Intervall zwischen der Intentionsmessung und der entsprechenden Verhaltensausführung ist. Ferner ist die TOPB vorrangig auf solche Verhaltensweisen anzuwenden, in denen der Einfluss kontrollierter kognitiver Prozesse hoch ist (JONAS und DOLL, 1996). Die Erklärung des habituellen Verhaltens bzw. von routinisierten Entscheidungen liegt indes außerhalb des Geltungsbereichs dieses theoretischen Ansatzes.

Eine weitere zentrale Annahme besteht darin, dass die Intention ihrerseits von drei indirekten Verhaltensdeterminanten beeinflusst wird, worunter die Einstellung zum Verhalten, die soziale Norm und die wahrgenommene Verhaltenskontrolle fallen (AJZEN, 1991). Das Einstellungskonstrukt impliziert hierbei eine globale instrumentelle und affektive Bewertung des Verhaltens, während die soziale Norm den sozialen Druck bzw. die wahrgenommenen Erwartungen wichtiger Bezugspersonen, ein bestimmtes Verhalten auszuführen oder nicht auszuführen, widerspiegelt. Die wahrgenommene Verhaltenskontrolle beeinflusst neben ihrer direkten Auswirkung auf das Verhalten auch die Absichtsbildung.

Die TOPB liefert darüber hinaus eine Erklärung für das Zustandekommen der drei indirekten Verhaltensdeterminanten, indem angenommen wird, dass diese auf bestimmten Überzeugungen basieren (AJZEN, 1991). Den beiden Konstrukten Einstellung zum Verhalten und der subjektiven Norm liegt ein erwartungswerttheoretisches Modell zugrunde. So wird die Einstellung gegenüber dem Verhalten als das Produkt aggregierter subjektiver Wahrscheinlichkeiten, mit denen bestimmte Verhaltenskonsequenzen einhergehen, und den dazugehörigen subjektiven Bewertungen konzeptualisiert. Nimmt eine Person also an, dass die Konsequenzen eines für sie relevanten Verhaltens mit hoher Wahrscheinlichkeit positiv und mit geringer Wahrscheinlich- 
keit negativ ausfallen werden, so resultiert hieraus eine positive Einstellung zur Verhaltensausführung. Hieraus wird ersichtlich, dass die Verhaltensüberzeugungen als multiplikativer Term aus Valenz und Instrumentalität in ihrer Konzeption dem erwartungswerttheoretischen Ansatz von VROOM (1966) entsprechen (vgl. AJZEN, 2008).

Analog zum Einstellungskonstrukt setzt sich die subjektive Norm ebenfalls aus zwei Komponenten zusammen - der normativen Überzeugung und der Konformitätsmotivation. Die normative Überzeugung bezieht sich auf die von einer Person wahrgenommenen Erwartungen relevanter Bezugspersonen, wie beispielsweise Eltern, Freunde oder Arbeitskollegen, während die Konformitätsmotivation die Bereitschaft von Personen, diesen Erwartungen nachzukommen, umfasst. Auch hierbei wird davon ausgegangen, dass das Produkt aus normativen Überzeugungen und der Konformitätsmotivation einen Einfluss auf die Verhaltensintention hat. Demzufolge sollte die Verhaltensabsicht umso größer sein, je mehr die wahrgenommenen Erwartungen relevanter Personen mit dem Verhalten konform sind.

Schließlich besteht die wahrgenommene Verhaltenskontrolle aus der multiplikativen Verknüpfung interner bzw. externer Kontrollüberzeugungen und der hierdurch als einfacher bzw. schwieriger wahrgenommenen Verhaltensausführung. Die wahrgenommene Verhaltenskontrolle ist dann hoch, wenn das Ausmaß von positiven internen Faktoren, wie beispielsweise von Kompetenzen und Ressourcen, hoch und der Einfluss negativer externer Variablen, wie beispielsweise von Hindernissen, gleichzeitig gering ist. Die Modellannahmen der TOPB sind in der Abbildung 3-3 veranschaulicht.

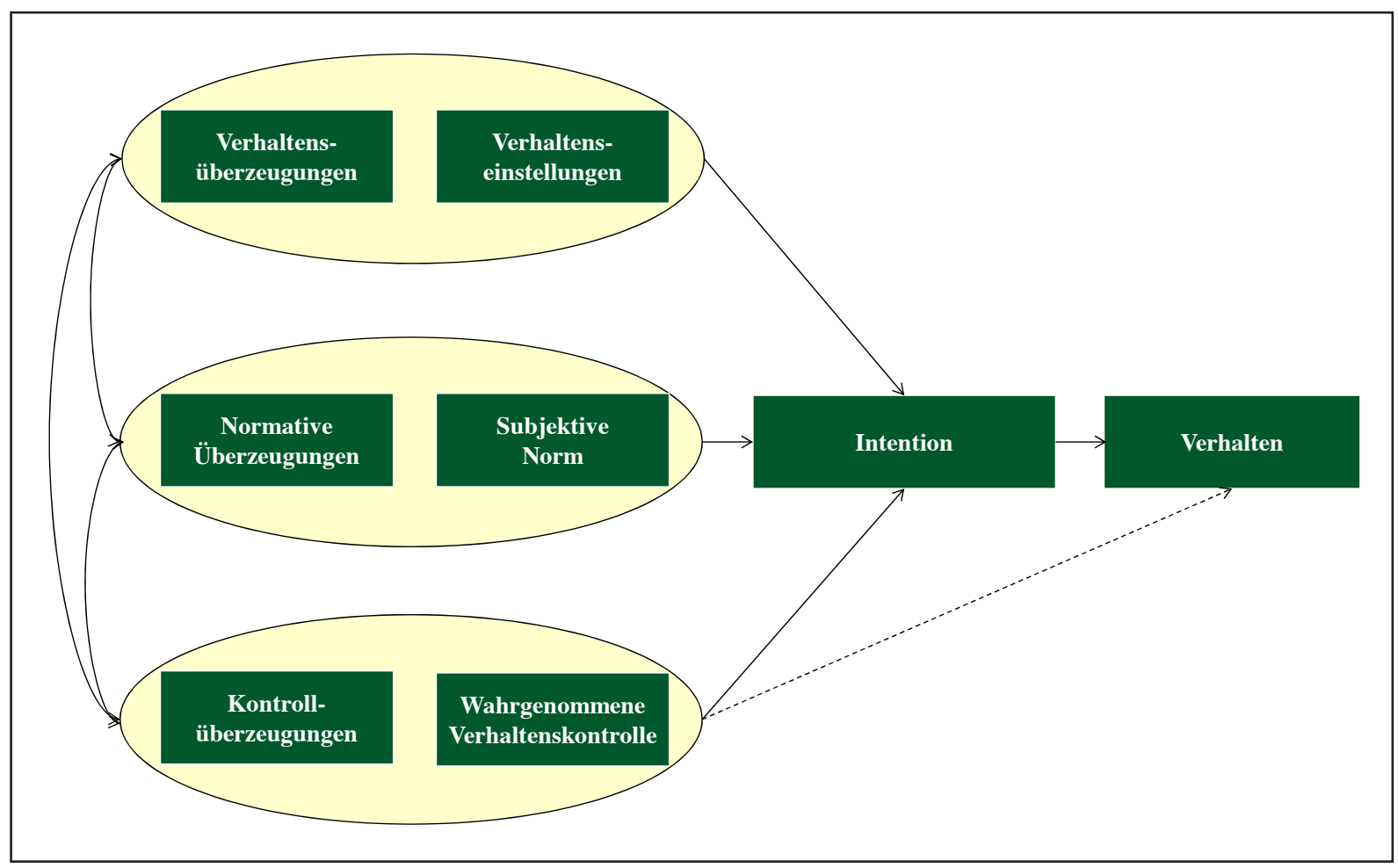

Abb. 3-3: Theorie des geplanten Verhaltens (Eigene Darstellung, 2011, in Anlehnung an AJZEN, 2008) 
Inzwischen liegt eine Reihe von Sammelreferaten und metaanalyitischen Studien zur Theorie des geplanten Verhaltens vor, in denen die theoretisch postulierten Zusammenhänge auch eine empirisch Bestätigung erfahren (z.B. SIX und ECKES, 1996; ARMITAGE und CONNER, 2001). Daraus lassen sich für die einzelnen Modellkomponenten durchschnittliche korrelative Ergebnisse ableiten (vgl. zusammenfassend AJZEN, 2008). Demzufolge liegt der korrelative Zusammenhang zwischen den Konstrukten Einstellung und Verhaltensintention zwischen $r=.45$ bis $r=.60$. Die Korrelation zwischen der wahrgenommenen Verhaltenskontrolle und der Verhaltensintention lässt sich indes auf $r=.35$ bis $r=.46$ beziffern. Als Schätzung für die Populationskorrelation zwischen der subjektiven Norm und der Verhaltensintention ergibt sich ein Wert von $r=.34$ bis $r=.42$. Aus dieser Gegenüberstellung wird zugleich deutlich, dass der korrelative Wert der Einstellung zum Verhalten im Vergleich zu den beiden anderen Prädiktoren am höchsten ausfällt und die Einstellung somit den größten individuellen Beitrag zur Erklärung der Verhaltensintention leistet. Für die multiple Korrelation der drei Verhaltensdeterminanten mit der Verhaltensintention ergeben sich Werte von $r=.63$ bis $r=.71$. Schließlich beläuft sich die Schätzung für die mittlere Korrelation zwischen der Verhaltensintention und dem Verhalten auf $\mathrm{r}=.41$. Die Effektstärken der hier dargestellten Ergebnisse liegen gemäß COHEN (1988) im mittleren bis hohen Bereich und bestätigen somit den bedeutenden Stellenwert der postulierten Einstellungs-Verhaltens-Relation. Allerdings geht aus diesen metaanalytischen Ergebnissen auch gleichzeitig hervor, dass der Intentions-Verhaltens-Zusammenhang nicht so hoch ausfällt, wie es gemäß den theoretischen Annahmen bei einem hohen Maß an wahrgenommener Verhaltenskontrolle zu erwarten wäre. Zwar versucht die Theorie des geplanten Verhaltens, mit dem Konstrukt der wahrgenommenen Verhaltenskontrolle Hindernisse und Barrieren bei der Verhaltensausführung zu erfassen, jedoch ist das Konzept zu global formuliert, um die einer Handlungsimplementierung zugrunde liegenden Kontrollprozesse adäquat abzubilden (JONAS und DOLL, 1996). Die Einschränkungen in der prädiktiven Kraft lassen sich zudem auf Ursachen zurückführen, die außerhalb der oben angeführten messtheoretischen und methodischen Unzulänglichkeiten liegen. So lässt sich die verringerte Intentions-Verhaltens-Beziehung u.a. dadurch erklären, dass motivationale und volitionale Prozesse, die als Mediatoren zwischen der Verhaltensintention und dem tatsächlichen Verhalten fungieren, in der TOPB bislang unberücksichtigt blieben (BAMBERG, 2002).

Die TOPB als fester Bestandteil der sozialpsychologischen Einstellungsforschung weist in ihrer Konzeption bedeutsame Gemeinsamkeiten mit der psychologischen Entscheidungsforschung auf, da beide Ansätze auf die Erklärung und Vorhersage von Verhalten mit unsicheren Konsequenzen fokussieren (PFISTER und KONERDING, 1996). Sowohl die TOPB als auch die zahlreichen SEU-Modelle sind als Varianten der allgemeinen Klasse von Erwartungs-Wert-Theorien zu sehen (JUNGERMANN et al., 2010). In beiden Ansätzen wird die Einstellung gegenüber einer bestimmten Verhaltensweise bzw. die Entscheidung für eine Handlung als Funktion der Beurteilung der damit einhergehenden Verhaltenskonsequenzen konzipiert. Zwei Aspekte die- 
ser Verhaltenskonsequenzen sind überdies für beide Ansätze zentral: ihre Unsicherheit und ihr Wert für die Person. Auch weitere Faktoren, die in der Entscheidungsforschung als ursächlich für eine Entscheidung unter Unsicherheit betrachtet werden, lassen sich auf die einstellungspsychologische Perspektive übertragen. So läst sich die Intention als eine interne Entscheidung für eine Verhaltensoption auffassen, die entscheidungstheoretisch einer hypothetischen Wahl entspricht. Die salienten Überzeugungen gehen mit den unsicheren Konsequenzen einer Option einher, während ihre Stärkeeinschätzungen den Wahrscheinlichkeiten entsprechen. Überdies sind die einstellungspsychologischen Bewertungen analog zu den Nutzenwerten der Konsequenzen zu sehen. Schließlich findet sich die Einstellungs-Verhaltens-Relation im entscheidungstheoretischen Zusammenhang zwischen Gesamtnutzen und realen Wahlen mit echten monetären Auszahlungen wieder.

Unter theoretischen Gesichtspunkten ist zudem hervorzuheben, dass zur TOPB trotz der zahlreichen Studien, in denen die kausalen Annahmen bestätigt wurden, auch Kritikpunkte und Erweiterungsvorschläge formuliert wurden (vgl. für einen Überblick: JONAS und DOLL, 1996). Der wichtigste Kritikpunkt betrifft die mangelnde Suffizienz der TOPB. Demzufolge entspricht die darin enthaltene Begrenzung nicht dem zugrunde liegenden Modellcharakter, da das Modell auf einem algebraischen Strukturmodell basiert und somit grundsätzlich um weitere Prädiktoren erweitert werden kann. Dieses ursprüngliche Prinzip hat AJZEN (1991) jedoch aufgegeben und postuliert stattdessen, dass die TOPB bei vorhandener theoretischer und empirischer Evidenz um weitere Konstrukte ergänzt werden kann.

Darüber hinaus wurde auch aus entscheidungstheoretischer Perspektive Kritik an der TOPB geübt (JONAS und DOLL, 1996). So dürfte es in Situationen mit mehr als einer Handlungsalternative zur Verhaltensvorhersage nicht ausreichen, lediglich die Einstellung zu einem einzigen Verhalten zu bestimmen, es müssten hierbei vielmehr die Einstellungen zu allen Handlungsalternativen bestimmt werden. Bei fehlender Berücksichtigung wird ECKES und SIX (1994) zufolge die prognostische Leistung der Theorie des geplanten Verhaltens in solchen Verhaltensbereichen verringert, in denen eine Vielzahl möglicher Verhaltensweisen mit recht ähnlichen Verhaltenskonsequenzen zur Auswahl steht, wie beispielsweise im Bereich des Konsumentenverhaltens. Anhand ihrer metaanalytischen Studie konnten die Autoren zeigen, dass bei einer dichotomen Verhaltenswahl mit genau zwei Alternativen sowohl die Einstellungs-Verhaltens-, Einstellungs-Intention- als auch die Intentions-Verhaltens-Korrelationen durchschnittlich höher ausfallen als bei einer polytomen Verhaltenswahl mit mehr als zwei Alternativen. Diese Eingrenzung erweist sich insofern als bedeutsam, als dass es aufgrund der bisherigen Forschungsergebnisse wenig wahrscheinlich ist, dass potenzielle Bewerber eine Präferenzreihung zwischen einer Vielzahl von Arbeitgebern vornehmen, sondern sich vielmehr jeweils für oder gegen ein bestimmtes Stellenangebot entscheiden (MOSER und ZEMPEL, 2004). 
Ein weiterer gewichtiger Erweiterungsvorschlag betrifft die Qualität der gebildeten Einstellungen (JONAS und DOLL, 1996). So sind Einstellungen, die auf direkten Erfahrungen mit dem Einstellungsobjekt basieren, für die entsprechende Verhaltensausführung prädiktiver als indirekt gebildete Einstellungen. Es liegen empirische Befunde dafür vor, dass diese Faktoren die Stärke des Zusammenhangs von Einstellung und Verhalten bzw. Einstellung und Intention moderieren (FAZIO und ZANNA, 1981). Ferner korrelieren Einstellungen, die sich auf Themen von hoher persönlicher Relevanz beziehen, höher mit dazugehörigen Intentionen als Einstellungen mit geringer persönlicher Relevanz (ARMITAGE und CHRISTIAN, 2003). Die berichteten Ergebnisse werden darauf zurückgeführt, dass die persönlich relevanten Einstellungen eine höhere Verfügbarkeit im Gedächtnis aufweisen oder durch mehr Kognitionen gestützt werden und dementsprechend stabiler sind. Dieses Erklärungsmuster findet sich im zentralen marketingwissenschaftlichen Konstrukt des Involvements wieder (vgl. TROMMSDORFF, 2009).

Darüber hinaus ist festzustellen, dass die wahrgenommene Verhaltenskontrolle in ihrer Konzeption beträchtliche Parallelen zum psychologischen Konstrukt der Selbstwirksamkeitserwartungen aufweist (JONAS und DOLL, 1996). Die theoretische Abgrenzung ist jedoch darin zu sehen, dass das Konzept der Selbstwirksamkeit auf interne Faktoren, wie die Einschätzung der eigenen Kompetenz, fokussiert, während die wahrgenommene Verhaltenskontrolle interne und externe Faktoren gleichermaßen berücksichtigt. Zugleich bestätigen die Ergebnisse der Metaanalyse von ARMITAGE und CONNER (2001), dass die Erweiterung der Theorie des geplanten Verhaltens durch das Konstrukt der Selbstwirksamkeit die Vorhersage von Intention und Verhalten verbessert.

Aus methodischer Perspektive ist ferner zu beachten, dass die überwiegende Zahl der zur Theorie des geplanten Verhaltens durchgeführten Studien korrelativer Natur sind, d.h. auf Querschnittdesigns basieren und infolgedessen keine Aussagen über die entsprechenden Kausalzusammenhänge zulassen. Auch die bisher wenigen experimentellen bzw. Längsschnittstudien ermöglichen insgesamt noch keine fundierte Bestätigung der postulierten kausalen Annahmen (ARMitAge und Christian, 2003). Ferner ist zu beachten, dass Studien, die Verhalten über Selbstberichte erfasst hatten, höhere Einstellungs-Verhaltens-Korrelationen aufwiesen als Studien, in denen das Verhalten beobachtet bzw. mittels objektiver Verhaltensmaße operationalisiert wurde (SIX und ECKES, 1996; ARMITAGE und CONNER, 2001).

Die Gültigkeit der TOPB konnte empirisch für diverse Fragestellungen bestätigt werden (vgl. für einen Überblick: AJZEN, 2008). Entsprechende Anwendungen auf das Bewerbungsverhalten finden sich bislang hingegen kaum (z.B. LEWANDOWSKI und LIEBIG, 2004; ARNOLD et al. 2006). Infolgedessen wird in dieser Arbeit systematisch der Frage nachgegangen, inwieweit die TOPB dazu geeignet ist, die Bewerbungsintention vorherzusagen und zu erklären. Gemäß dem einstellungspsychologischen Modell wird die Bewerbungsabsicht als das zentrale zu erklärende Kriterium der Arbeitgeberwahl konzipiert. 
Die Modelldeterminanten Verhaltensintention, Einstellung zum Verhalten, wahrgenommene Verhaltenskontrolle sowie subjektive Norm werden inhaltlich an die zugrunde liegende Forschungsfrage angepasst, indem sie entsprechend als Bewerbungsabsicht, Arbeitgeberattraktivität, Erfolgswahrscheinlichkeit der Bewerbung sowie organisationales Prestige gemessen werden. Der Zusammenhang zwischen der Bewerbungsabsicht und dem tatsächlichen Bewerbungsverhalten wird hier allerdings nicht aufgearbeitet, da das primäre Forschungsziel dieser Arbeit darin besteht, das Zustandekommen der Bewerbungsintention möglichst gut zu erklären und die hierfür ursächlichen Determinanten eindeutig zu identifizieren.

\subsection{Fazit}

In den vorangegangenen Kapiteln wurden die wichtigsten Theorien zur Erklärung der Arbeitgeberwahl vorgestellt. Die theoretischen Ausführungen wurden um weitere in der Literatur vorgeschlagene Modelle, wie beispielsweise das Modell der Person-Organisation-Passung, ergänzt. Insgesamt wird dabei deutlich, dass jedes dieser theoretischen Ansätze zwar bestimmte Phänomene der Arbeitgeberwahl zu erklären vermag, diese jedoch auf teils sehr unterschiedlichen theoretischen Annahmen und Operationalisierungsstrategien beruhen. Ein wesentliches Forschungsziel dieser Arbeit ist vor diesem Hintergrund auch darin zu sehen, die vorliegenden interdisziplinären Erkenntnisse in einer integrativen Rahmenkonzeption abzubilden, um ein möglichst breites Spektrum des Informations- und Entscheidungsverhaltens von potenziellen Bewerbern zu erklären. Unter forschungsökonomischen Gesichtspunkten muss das Erklärungsmodell der Arbeitgeberwahl zugleich aus einer überschaubaren Faktorenanzahl bestehen, sodass die Integration jedes Konstruktes ein stetiges Abwägen seines Erklärungsbeitrags erfordert. 


\section{KAPITEL 4}

\section{Modellierung der Arbeitgeberwahl}

In den vorangegangenen Kapiteln wurden die wesentlichen theoretischen Erkenntnisse und empirischen Befunde zur Arbeitgeberwahl dargestellt und kritisch diskutiert. Bei einer näheren Betrachtung der berichteten Befunde wird insgesamt deutlich, dass die Forschungsfrage, anhand welcher Arbeitsplatzmerkmale und Mechanismen sich potenzielle Bewerber für ein bestimmtes Stellenangebot entscheiden, bislang noch nicht hinreichend beantwortet werden konnte (MOSER und ZEMPEL, 2006; BOSWELL, 2012). Hieraus lässt sich das primäre Forschungsziel dieser Arbeit ableiten, ein theoretisch fundiertes Rahmenmodell zu entwickeln, das es ermöglicht, das Informations- und Entscheidungsverhalten von potenziellen Bewerbern genauer zu beschreiben und vor allem zu erklären. Insgesamt mangelt es in der Forschung zur Arbeitgeberwahl bislang an Studien, die bereits vorliegende marketingwissenschaftliche und psychologische Erkenntnisse miteinander verbinden. Neben der Integration neuer, überwiegend sozial- und persönlichkeitspsychologischer Konstrukte besteht ein wesentliches Forschungsziel somit darin, ausgewählte fundierte Konzepte der beiden Forschungsdisziplinen in einem theoriegeleiteten Modell zu verknüpfen, um so zu einer besseren Erklärung des Informations- und Entscheidungsverhaltens potenzieller Bewerber beizutragen. Dieses übergeordnete Forschungsziel wird in dieser Arbeit auf drei Teilmodelle aufgegliedert, wobei in jedem Teilmodell entsprechende Determinanten des Informations- und Entscheidungsverhaltens von potenziellen Bewerbern herausgearbeitet werden. Ein solches Vorgehen bietet sich auch deshalb an, da die im Rahmenmodell berücksichtigten Phasen in den meisten einschlägigen Studien getrennt thematisiert werden.

Im Folgenden wird zunächst der Frage nachgegangen, wie das Informationsverhalten potenzieller Bewerber gestaltet ist. Hier geht es insbesondere darum, den Bekanntheitsgrad und die Nutzungsintensität einzelner Personalmarketingmaßnahmen zu identifizieren und sie im zweiten Schritt auf ihre wahrgenommene Nützlichkeit hin zu überprüfen. Nachfolgend wird untersucht, welche Arbeitsplatzmerkmale potenzielle Bewerber in mittelständischen Unternehmen der Ernährungsindustrie als bedeutsam einschätzen und inwieweit ihre Bewertungen von den zugrunde liegenden persönlichkeitsspezifischen Merkmalen abhängig sind. Schließlich soll anhand eines Kausalmodells die Frage erörtert werden, welche Determinanten die Bewerbungsabsicht beeinflussen. Zu jedem dieser drei Forschungsfragen wird anhand der jeweiligen Hypothesen ein separates Teilmodell aufgestellt, das jeweils als Grundlage für die empirische Untersuchung dient. 


\subsection{Informationsverhalten potenzieller Bewerber}

Die externe Bewerberansprache kann grundsätzlich anhand von unterschiedlichen Maßnahmen erfolgen, die sich zugleich in der von potenziellen Bewerbern bevorzugten Spezifität, Informationsmenge und auch ihrer Wirksamkeit deutlich voneinander unterscheiden (vgl. z.B. GATEwOOD et al., 1993; COBER et al., 2004; ALLEN et al., 2007; COLLINS, 2007; KANNING et al., 2009). Eine Untersuchung von MOSER (1995) konnte in diesem Zusammenhang auch langfristige Effekte der eingesetzten Kommunikationsmaßnahmen offenlegen. So konnte der Autor zeigen, dass die zur externen Bewerberansprache verwendeten Instrumente unter anderem einen Einfluss auf die spätere Leistung, das Commitment und die Einstellung zur Arbeit der Mitarbeiter haben. Die Entscheidung für den Einsatz bestimmter Instrumente ist vor diesem Hintergrund in starkem Maße von den für die Personalakquisition relevanten Zielgruppensegmenten abhängig, wofür wiederum eine genaue Kenntnis des Informationsverhaltens potenzieller Bewerber unabdingbar ist (KIRCHGEORG und MÜLLER, 2011). Das Informationsverhalten potenzieller Bewerber wird in dieser Arbeit in Anlehnung an MALMENDIER (2006) in zwei separate Phasen gegliedert: Informationsbeschaffung und Informationsverarbeitung. Die Informationsbeschaffung umfasst hierbei den Bekanntheitsgrad und Nutzungsintensität der einzelnen Kommunikationsmaßnahmen, während die Informationsverarbeitung auf ihre Bewertung bzw. Präferenz fokussiert. Eine detaillierte Betrachtung der Informationsbeschaffung ist deshalb erforderlich, da die unterschiedlichen Rekrutierungsquellen nur dann wirksam sein können, wenn sie die potenziellen Bewerber auch tatsächlich erreichen (COLLINS, 2007). Gemäß dem oben geschilderten Suchkostenansatz hängt die individuelle Nutzung der einzelnen Kommunikationsmaßnahmen im Wesentlichen von den damit einhergehenden Suchkosten ab. Ein potenzieller Bewerber wird demzufolge eher solche Instrumente des Personalmarketings beanspruchen, die mit einem vergleichsweise geringen Aufwand, zu dem sowohl finanzielle als auch zeitliche Faktoren zählen, einhergehen. Die mit einer Kommunikationsmaßnahme verbundenen Suchkosten sind potenziellen Bewerbern i.d.R bereits im Vorfeld bekannt (TEUFER, 1999). Wie im Abschnitt 3.1.1 erläutert, eignet sich der informationsökonomische Suchkostenansatz auch aus der Bewerbersicht nur bedingt zur umfassenden Erklärung ihrer Informationsbeschaffungsaktivitäten, sodass hier ergänzend auf das Involvementkonstrukt zurückgegriffen wird.

Obgleich es unterschiedliche Konstruktdefinitionen des Involvements gibt, stimmen sie inhaltlich darin überein, dass es sich hierbei um den „Aktivierungsgrad bzw. die Motivstärke zur objektgerichteten Informationssuche, -aufnahme, -verarbeitung und -speicherung" handelt (TROMMSDORFF, 2009: 49). Der Konstruktdefinition folgend lässt sich das Involvement im Ergebnis zwar eindimensional anhand der individuellen Aktivierungsintensität abbilden, die das Involvement konstituierenden Faktoren beruhen jedoch auf einer mehrdimensionalen Konstellation. So resultiert die Aktivierungsintensität in Bezug auf das Entscheidungsobjekt bzw. die Entscheidungssituation aus der Interaktion von insgesamt fünf Determinanten, worunter personen-, produkt-, situations-, medien- sowie botschaftsspezifische Faktoren fallen (TROMMS- 
DORFF, 2009). Personenspezifisches Involvement wird von individuellen Kenntnissen, Erfahrungen und Persönlichkeitseigenschaften, worunter beispielsweise auch die persönlichen Werte, Ziele, Einstellungen und Motive fallen, determiniert. Die Höhe des Involvements, das durch ein bestimmtes Objekt ausgelöst wird, ist somit davon abhängig, inwieweit es die zentralen Kenntnisse und Persönlichkeitseigenschaften eines Konsumenten trifft. Im Produktinvolvement drückt sich hingegen die persönliche Bedeutung aus, die ein bestimmtes Produkt oder eine bestimmte Produktart für den Konsumenten besitzt. Situationsspezifisches Involvement lässt sich indes anhand der zeitlichen Nähe zur Entscheidungssituation bestimmen, in der sich der Konsument im Zuge seiner Entscheidungsfindung befindet. So steigt das Involvement erst dann auf ein entsprechendes Niveau an, wenn die (Kauf-)Entscheidung aktuell ist und gleichzeitig das entsprechende Problembewusstsein für die bestehende Entscheidungssituation auslöst. Das Botschaftsinvolvement wird dadurch begründet, dass die Botschaft an sich mehr oder weniger interessant sein kann, sodass der Fokus hierbei weniger auf die subjektive Bedeutung der Produkteigenschaften, sondern vielmehr auf den Unterhaltungswert der Werbung gelenkt wird. Schließlich wird im Rahmen des Medieninvolvements postuliert, dass Medien durch ihre spezifische Kommunikationsweise die Höhe des Involvements beeinflussen. Die oben dargestellte Faktorenkonstellation beeinflusst ihrerseits die Relevanz des Entscheidungsobjektes bzw. der Entscheidungssituation für das Individuum und damit das daraus resultierende individuelle Involvement. Folglich ist das Involvement nicht als Ursache des Informationsverhaltens zu sehen, sondern nimmt vielmehr eine vermittelnde Rolle in diesem Zusammenhang ein (KROEBER-RIEL et al., 2009).

Für die Analyse des Bekanntheitsgrades und der Nutzungsintensität einzelner Kommunikationsmaßnahmen erweist sich das Involvement als Prädiktor der beiden Informationsbeschaffungskriterien als besonders relevant. So postuliert TROMMSDORFF (2009), dass ein niedriges Involvement eine passive Informationssuche begünstigt, während ein hohes Involvement mit einem höheren Aktivitätsniveau einhergeht. Analog hierzu lassen sich auch die Instrumente des Personalmarketings nach dem jeweils vorausgesetzten Aktivitätsniveau unterscheiden. Stellenanzeigen oder Imagebroschüren gehen eher mit einer passiven Informationsaufnahme einher, während Besuche von Firmenmessen oder Unternehmensexkursionen eine aktive Informationssuche erfordern (MALMENDIER, 2006). Dieser Annahme folgend differenziert SÜSS (1996) in seinem Modell zwischen Kommunikationsmaßnahmen, die entweder in der Low-Involvement-Phase oder aber in der Präferenzbildungsphase beansprucht werden sollten, wobei seine Annahme aufgrund der fehlenden empirischen Überprüfung in seiner Studie einen rein hypothetischen Charakter beibehält. Insgesamt liegen damit im Bereich der Informationsbeschaffung bislang keine fundierten theoretischen Ansätze vor, sodass dieses Teilmodell durch einen eher explorativen Forschungscharakter gekennzeichnet ist. Dabei wird untersucht, inwieweit soziodemographische und persönlichkeitsspezifische Merkmale potenzieller Bewerber mit dem Bekanntheitsgrad und der Nutzungsintensität einzelner Personalmarketingmaßnahmen zusammenhängen. 
Analog zur vorangehenden Phase der Informationsbeschaffung fungiert das Involvement auch im Rahmen der Informationsverarbeitung als ein bedeutsames Konstrukt. So wird im ELMModell von PETTY und CACIOPPO (1986) postuliert, dass die Art der Informationsverarbeitung im Wesentlichen vom individuellen Involvement abhängt. Hohes Involvement geht demzufolge mit einer rationalen Verarbeitung aller verfügbaren Informationen einher, während bei einem niedrigen Involvement eher Signale und Heuristiken in den Vordergrund der Informationsverarbeitung rücken. Wie oben beschrieben, unterscheiden sich auch die Instrumente des Personalmarketings in ihrer Spezifität und Informationsmenge, sodass ihre Bewertung durch potenzielle Bewerber von dem zugrunde liegenden individuellen Involvement abhängig sein sollte. Es wird in diesem Teilmodell somit auch der Frage nachgegangen, inwieweit die Nützlichkeitsbeurteilung der Kommunikationsmaßnahmen anhand der soziodemographischen und persönlichkeitsspezifischen Merkmale potenzieller Bewerber vorhergesagt werden kann.

\subsection{Differenzielle Wirkung des Arbeitgeberimages}

Mit dem Begriff Arbeitgeberimage wird in dieser Arbeit der Gesamteindruck eines Unternehmens in seiner Rolle als Arbeitgeber beschrieben, den potenzielle Bewerber aus der subjektiven Bewertung von beobachtbaren und nicht beobachtbaren Arbeitsplatzmerkmalen gewinnen. Gemäß der marketingwissenschaftlichen Definition wird das Arbeitgeberimage gleichzeitig als eine mehrdimensionale und ganzheitliche Grundlage der Einstellung in Form einer Arbeitgeberattraktivitätseinschätzung eines bestimmten Arbeitgebers betrachtet. Obgleich die Relevanz des Arbeitgeberimages innerhalb der Arbeitgeberwahl inzwischen als erwiesen gilt, liegt dazu bislang keine studienübergreifende Konstruktoperationalisierung vor. Die entsprechende Auswahl hat indes häufig einen rein praxisorientierten Charakter und erfolgt dabei größtenteils ohne jegliche theoretische Annahmen. Ausschlaggebend für die hier getroffene Auswahl von Arbeitsplatzmerkmalen war demgegenüber die Frage, welche Bewerbererwartungen im Strategic Image vorliegen, da hierfür gemäß der Image-Theorie sichergestellt ist, dass sie grundsätzlich entscheidungsrelevant sind. In diesem Zusammenhang wurde zugleich angenommen, dass sich die Bewerbererwartungen unmittelbar auf die Attributgewichtung auswirken, da das Strategic Image mit den Attributen des zu beurteilenden Objektes korrespondiert (BALDERJAHN und WIEDEMANN, 1999).

Folgende Arbeitsplatzmerkmale wurden aufgrund dieser Prämissen berücksichtigt: Bezahlung, Karriere- und Aufstiegschancen, Vereinbarkeit von Beruf und Familie bzw. Privatleben sowie Arbeitsplatzsicherheit. Ein wesentliches Forschungsziel besteht hierbei darin, empirisch zu überprüfen, ob und inwieweit die oben dargestellten Arbeitsplatzmerkmale das Entscheidungsverhalten von potenziellen Bewerbern beeinflussen. Daneben werden Gütesiegel in das Teilmodell aufgenommen, um ihre postulierte Wirkung als Signal innerhalb der Arbeitgeberwahl 
zu überprüfen. Um zu analysieren, ob der Einfluss der Arbeitsplatzmerkmale auf die Wahlwahrscheinlichkeit eines Stellenangebotes mit den persönlichkeitsspezifischen Merkmalen variiert, werden zusätzlich die Berufsorientierungen potenzieller Bewerber in diesem Teilmodell berücksichtigt.

\subsubsection{Karriere- und Aufstiegschancen}

Das Arbeitsplatzmerkmal Karriere- und Aufstiegschancen wird insgesamt als ein zentraler Bestandteil des Arbeitgeberimages betrachtet. Es wird sowohl in den praxisorientierten als auch in den wissenschaftlichen Studien als bedeutsam und entscheidungsrelevant eingeschätzt (z.B. WiLTINGER, 1997; LEWANDOWSKI und LIEBIG, 2004; KIRCHGEORG und GÜNTHER, 2006; BÖTTGER, 2012). Dabei stellen RosEnSTIEL und NERDINGER (2000) heraus, dass die Aufstiegschancen eine wesentliche Grundlage für die Selbstselektion und die wahrgenommene Person-Organisation-Passung darstellen, woraus sie entsprechenden Handlungsbedarf für das Personalmarketing ableiten. Ferner weisen die Ergebnisse ihrer Untersuchung darauf hin, dass Arbeitnehmer die Organisation bereits nach kurzer Zeit verlassen, wenn sie wider Erwarten keine Möglichkeiten der beruflichen Entwicklung im Unternehmen erkennen. Dieser Befund steht zugleich im Einklang mit den Annahmen des im Abschnitt 3.5 dargestellten ASA-Modells.

\subsubsection{Vereinbarkeit von Beruf und Familie/Privateben}

Dem Arbeitsplatzmerkmal Vereinbarkeit von Beruf und Familie bzw. Privatleben wird in der Forschung zur Arbeitgeberwahl ebenfalls eine hohe Bedeutung beigemessen. So kamen BouRHIS und MeKKAOUI (2010) in ihrer Studie zu dem Ergebnis, dass potenzielle Bewerber vor allem solche Unternehmen als attraktive Arbeitgeber bewerten, in denen organisationale Instrumente zur Förderung der Work-Life-Balance weit verbreitet sind. Einen weiteren Beleg für die Bedeutsamkeit dieses Arbeitsplatzmerkmals liefert die Untersuchung von HONEYCUTT und Rosen (1997), in der Unternehmen mit flexiblen Aufstiegsmöglichkeiten und Arbeitsbedingungen von MBA-Studierenden positiver beurteilt wurden.

\subsubsection{Bezahlung}

Die empirische Forschung zur Bedeutung des Arbeitsplatzmerkmals Bezahlung bringt sehr heterogene Ergebnisse hervor. Während einige Untersuchungen der Bezahlung eine vergleichsweise hohe Bedeutung beimessen, wird diese in anderen Studien als weniger entscheidungsrelevant eingestuft (vgl. Abschnitt 2.5.1). Die uneinheitlichen und teilweise widersprüchlichen 
Forschungsergebnisse lassen sich zum einen auf theoretische Erklärungsansätze, wie z.B. auf die Forschung zum Wertewandel, zurückführen (ROSENSTIEL und NERDINGER, 2000). Zum anderen erweisen sich die methodischen Probleme als ursächlich für die vorhandenen Ergebnisse. Gleichzeitig wird in der marketingwissenschaftlichen Forschung die Ansicht vertreten, dass ,die Entgeltpolitik im Personalmarketing das Instrument mit der schnellsten und unmittelbarsten Wirkung auf Personalimage, Attraktivität und Präferenz eines Unternehmens“ darstellt (SIMON et al., 1995: 199). Da die bisherigen Itemformulierungen, wie beispielsweise „attraktive Gesamtvergütung“ oder „hohes Einstiegsgehalt“, zu abstrakt formuliert sind und die Angabe einer absoluten Gehaltshöhe aufgrund der deutlich abweichenden Werte zwischen den einzelnen Branchenzweigen bzw. Unternehmen nicht aussagekräftig erschienen, wird hier die Vergütungsstruktur der Operationalisierung zugrunde gelegt. Demzufolge wird ein branchenübliches, durchschnittliches Festeinkommen einer leistungsabhängigen variablen Vergütung gegenübergestellt.

\subsubsection{Arbeitsplatzsicherheit}

Die Bedeutung der Arbeitsplatzsicherheit konnte in mehreren Untersuchungen zur Arbeitgeberwahl hinreichend nachgewiesen werden. So konnten LANG VON-WINS et al. (1995) zeigen, dass die Entscheidung eines potenziellen Bewerbers für eine Organisation insbesondere von der subjektiven Bedeutung der Arbeitsplatzsicherheit beeinflusst wird. Den Stellenwert der Arbeitsplatzsicherheit verdeutlichen auch die Ergebnisse der Studie von AIMAN-SMITH et al. (2001), in der sich die Arbeitsplatzsicherheit neben der Vergütung und der CSR-Orientierung des Unternehmens als wesentlicher Prädiktor für die Arbeitgeberattraktivität erweist. Ferner untersuchten SCHWOERER und ROSEN (1989) in ihrer experimentellen Studie die Wirkung unterschiedlicher Kombinationen von Bezahlung und Arbeitsplatzsicherheit auf die Bewerbungsbereitschaft. Die Vergütung wurde über die beiden Ausprägungen durchschnittlich oder überdurchschnittlich erfasst, während die Arbeitsplatzsicherheit entweder als willkürliche oder als zu begründende Kündbarkeit operationalisiert wurde. Es zeigte sich, dass sich die Bezahlung durchweg positiv auf die Bewerbungsbereitschaft auswirkt, wohingegen die Ergebnisse für die Konsequenz des Arbeitsplatzmerkmals Arbeitsplatzsicherheit differenzierter ausfallen: So bewirkt die willkürliche Kündbarkeit bei durchschnittlicher Bezahlung zwar die geringste Bewerbungsbereitschaft, sie wird jedoch bei überdurchschnittlicher Bezahlung mehr als kompensiert, sodass gerade diese Kombination insgesamt zur größten Bewerbungsbereitschaft führt. Die in der Studie verwendete Merkmalsausprägung der willkürlichen Kündbarkeit stellt eine arbeitsrechtliche Besonderheit des Arbeitsmarktes in den USA dar, wobei hier die Übertragung auf andere Bereiche unsicherer Beschäftigung, wie beispielsweise zeitlich befristete Verträge, plausibel erscheint (vgl. SCHULER und MOSER, 1993). 


\subsubsection{Gütesiegel}

Ein klassisches Instrument der externen Bewerberansprache stellt nach wie vor die Stellenanzeige in unterschiedlichen Print- und Online-Medien dar (MARCUS, 2011). Betrachtet man die von Unternehmen in einschlägigen Medien geschalteten Stellenanzeigen, so lassen sich diese in drei grobe Kategorien einordnen (SCHMIDTKE, 2002). Demzufolge ist die erste Kategorie dadurch gekennzeichnet, dass die Arbeitsplatzmerkmale darin gar nicht beleuchtet werden, während in der zweiten Kategorie ausschließlich auf beobachtbare Arbeitsplatzmerkmale eingegangen wird. Demgegenüber werden innerhalb der dritten Kategorie zwar auch nicht beobachtbare Arbeitsplatzmerkmale in den Vordergrund gestellt, allerdings werden sie nicht glaubhaft belegt. Stellenanzeigen, in denen die nicht beobachtbaren Arbeitsplatzmerkmale durch glaubhafte und greifbare Signale untermauert werden, finden sich hingegen bislang eher selten. Problematisch an dieser Situation ist zugleich, dass ein Unternehmen dadurch gleichzeitig den zentralen Imagebildungsprozess weitgehend potenziellen Bewerbern überlässt, anstatt seine vorhandenen Stärken bewusst und gezielt einzusetzen (TROST, 2012). Diese Problematik betrifft kleine und mittelständische Unternehmen in besonderem Maße, da sie häufig nicht über ein ausgeprägtes Image verfügen, weshalb bei externen Zielgruppen des Personalmarketings grundsätzlich auch von einem vergleichsweise hohen Anteil an empfundener Unsicherheit bei der Arbeitgeberwahl auszugehen ist (KAY et al., 2010). Ein Lösungsansatz besteht hierbei darin, verstärkt auf die Gestaltung von Signalen für nicht beobachtbare Arbeitsplatzmerkmale zu setzen und auf diesem Wege die Entscheidungsunsicherheit von potenziellen Bewerbern zu reduzieren (SCHMIDTKE, 2001). Ein solches Signal stellen grundsätzlich Gütesiegel dar, die darauf abzielen, die Vertrauensbildung zwischen zwei unbekannten Transaktionspartnern zu fördern (NOLL und WINKLER, 2004). Demgemäß können Gütesiegel, die von anbieterunabhängigen, vertrauenswürdigen Instanzen nach klar definierten Prüfkriterien vergeben werden, den Reputationsaufbau eines Unternehmens fördern. Zudem fungieren Gütesiegel als Signale, die dazu dienen, die bestehenden Informationsasymmetrien abzubauen und somit analog zur Sucheigenschaft die Qualitätsunsicherheit des Konsumenten bei Erfahrungs- und Vertrauenseigenschaften abzumindern bzw. zu beseitigen (TROMMSDORFF, 2009). Für den Konsumenten erfüllen sie insgesamt zwei wesentliche Funktionen: Zum einen informieren sie über nicht beobachtbare Produkteigenschaften, wie beispielsweise die Qualität eines Produktes, und zum anderen vermitteln sie einen Wert an sich, indem sie das Prestige des zugrunde liegenden Produktes erhöhen (SAMMER und WÜSTENHAGEN, 2006). Ob ein Gütesiegel jedoch tatsächlich eine (Kauf-)Entscheidung beeinflusst, hängt einerseits von seiner Bekanntheit und Glaubwürdigkeit und andererseits von seinem Aussagewert bzw. Informationsgehalt sowie seiner relativen Bedeutung im Vergleich zu anderen verfügbaren Produktmerkmalen ab (NOLL und WINKLER, 2004).

Überträgt man die obigen Annahmen auf das Personalmarketing, so lässt sich schlussfolgern, dass Gütesiegel bestimmte nicht beobachtbare Arbeitsplatzmerkmale, die ihrerseits zu den Erfahrungs- und Vertrauenseigenschaften eines Arbeitgebers zuzuordnen sind, in Sucheigen- 
schaften transformieren sollten. Infolgedessen sollen sie bei der externen Bewerberansprache vorrangig dazu dienen, das Arbeitgeberimage zu verbessern und die Rekrutierungseffektivität zu erhöhen (vgl. HUF, 2007; TAVAKKOLI, 2009; NAUNDORF und SPENGLER, 2012). Dieser wird von den überwiegend kommerziellen Anbietern allerdings ad hoc angenommen, ohne ausreichend überprüft worden zu sein (SCHERM und SÜSS, 2010). Bislang existieren nur vereinzelte wissenschaftliche Studien, aus denen sich Hinweise hinsichtlich dieses Wirkungszusammenhangs ableiten lassen. So lässt sich die Wirkung von Gütesiegeln anhand der Studie CABLE und TURBAN (2003) veranschaulichen, in der gezeigt werden konnte, dass eine hohe Platzierung in einem Arbeitgeber-Ranking (,,Fortune“) die Vertrautheit mit einem Unternehmen und zugleich seine Reputation positiv beeinflusst. In einer ähnlichen Weise konnten HIGHHOUSE et al. (2007) zeigen, dass sich die Arbeitgeber-Rankings positiv auf die Wahrnehmung bestimmter organisationaler Merkmale, wie beispielsweise der Innovationsstärke eines Unternehmens, auswirken. Bis auf vereinzelte Studien liegen in diesem Bereich bislang allerdings keine weiterführenden Erkenntnisse vor. Infolgedessen stehen hier drei wesentliche Forschungsfragen im Fokus. Zum einen wird der Frage nachgegangen, ob es sich bei den Gütesiegeln überhaupt um ein entscheidungsrelevantes Attribut innerhalb des Arbeitgeberimages eines Unternehmens handelt. Dabei werden auch die beiden zugrunde liegenden Wirkungsvoraussetzungen - ihr Bekanntheitsgrad und ihre Relevanz - innerhalb dieses Teilmodells berücksichtigt. In diesem Zusammenhang wird zugleich überprüft, inwieweit Gütesiegel als Signale ihre beiden Hauptfunktionen im Personalmarketing - die Informations- und die Wertfunktion - zu erfüllen vermögen. Zweitens soll ihr Stellenwert in Relation zu den oben dargestellten Arbeitsplatzmerkmalen festgestellt werden. Und drittens wird geprüft, inwiefern die postulierte Wirkung von Gütesiegeln von persönlichkeitsspezifischen Merkmalen potenzieller Bewerber, hier Berufsorientierungen, abhängt. So konnten MOSER et al. (1999) in ihrer experimentellen Studie zeigen, dass potenzielle Bewerber ein Unternehmen besser bewerten, wenn die in der Stellenanzeige illustrierten Werte mit ihren eigenen Berufsorientierungen übereinstimmen. Um dieser Frage systematisch nachzugehen, werden die beiden in der Unternehmenspraxis inzwischen recht weit verbreiteten Gütesiegel „Top Job“ und ,,audit berufundfamilie“ in das Teilmodell integriert.

\subsubsection{Berufsorientierungen}

Um den Einfluss interindividueller Faktoren festzustellen, greifen die meisten marketingwissenschaftlichen Studien auf die Segmentierung mittels einer Clusteranalyse zurück, was allerdings hinsichtlich der Ergebnisvalidierung z.T. kritisch betrachtet wird (BOSSOW-THIES und CLEMENT, 2009). Der hier gewählte Segmentierungsansatz basiert demgegenüber auf sog. Berufsorientierungen, deren differenzielle prognostische Validität in vielen empirischen Studien hinreichend belegt werden konnte (vgl. für einen Überblick: BLICKLE, 1999). Berufsorientierungen werden hierbei als ,weitgehend stabile und langfristige berufliche Bestrebungen von Personen“ definiert (MAIER et al., 2009: 105). Innerhalb dieses Konstruktes werden drei Be- 
rufsorientierungen unterschieden: Karriereorientierung, Freizeitorientierung sowie alternatives Engagement (BLICKLE, 1999). Die Karriereorientierung zeichnet sich durch ein Streben nach beruflichem Aufstieg und persönlicher Einflussnahme auf organisationale Entscheidungen aus, wofür karriereorientierte Personen eine höhere Anstrengungsbereitschaft aufzubringen bereit sind. Demgegenüber spiegelt sich in der Freizeitorientierung eine höhere Gewichtung der eigenen Freizeit im Vergleich zur Arbeitszeit wider. Darüber hinaus zielen freizeitorientierte Personen auf eine gesicherte Position mit geregelter Arbeitszeit und angenehmer Arbeitsatmosphäre ab. Sowohl die Karriere- als auch die Freizeitorientierung lassen sich einer materialistischen Werthaltung zuordnen, wobei sie sich zugleich auch grundlegend in ihrem strukturellen Fokus unterscheiden. Während die Karriereorientierung auf extrinsische Anreize, wie beispielsweise ein hohes Gehalt und Einflussnahme auf organisationale Entscheidungen, abzielt, geht es bei der Freizeitorientierung vorrangig um die Realisierung eigener Interessen außerhalb der Arbeitszeit. Alternatives Engagement umfasst im Gegensatz dazu eine postmaterialistische Werthaltung, die sich darin niederschlägt, dass alternativ engagierte Personen viel eher bereit sind, auf extrinsische Anreize zu verzichten. Im Vordergrund steht hierbei indes das Streben nach Selbstverwirklichung und nach „Idealen einer besseren Welt in der Arbeit“ (MAIER et al., 2009: 105). Analog zu den karriereorientierten Personen sind jedoch auch alternativ engagierte Personen bereit, einen hohen Arbeitseinsatz zu erbringen.

Die Verwendung dieses Konstruktes liegt in seiner theoretischen Abgrenzung zu den beiden verwandten Konstrukten - den persönlichen beruflichen Zielen einerseits und den Wertvorstellungen andererseits - begründet. So sind persönliche berufliche Ziele mehr der volitionalen Ebene zuzuordnen, während sich Berufsorientierungen eher durch ihren motivationalen Charakter auszeichnen (ROSENSTIEL und NERDINGER, 2000). Persönliche berufliche Ziele fokussieren somit auf vergleichsweise konkrete Handlungsergebnisse und lassen sich als ,ideographische Ausdrucksformen von Berufsorientierungen“ auffassen (BLICKLE, 1999: 18). Demgegenüber nehmen Werthaltungen eine den Berufsorientierungen übergeordnete Ebene ein, da sie als abstrakte und zeitlich stabile Konzeptionen des Wünschenswerten gelten und somit auch die Auswahl verfügbarer Handlungen und Ziele beeinflussen (KLUCKHOHN, 1951, zit. in ROSENSTIEL und NERDINGER, 2000). Während die beruflichen Werthaltungen einen allgemeinen Orientierungsrahmen für das berufliche Handeln bilden, können Berufsorientierungen als übergeordnete Zielintentionen verstanden werden, die ihrerseits konkrete berufliche Entscheidungen, wie beispielsweise die Arbeitgeberwahl, beeinflussen (MAIER et al., 1994). Ein wesentlicher Unterschied zwischen diesen beiden Konstrukten ist überdies darin zu sehen, dass Werte als Konzeptionen des Wünschenswerten ebenso abstrakt wie zeitlich stabil sind, während Berufsorientierungen sich auf ganz konkrete Bedingungen des Arbeitslebens beziehen und sich als Reaktion auf diese im Rahmen von Sozialisationsprozessen auch ändern können (ROSENSTIEL und NerdingER, 2000). Die theoretische Nähe beider Konstrukte lässt sich insgesamt daran erkennen, dass alle Aspekte, die im Rahmen des Konstruktes der Berufsorientierungen erfragt werden, in einer ähnlichen Form auch in den Instrumenten zur Erhebung von Werthaltungen 
zu finden sind (BLICKLE, 1999). Umgekehrt werden nicht sämtliche Werthaltungen mittels des Konstruktes der Berufsorientierungen abgebildet, da dieses ursprünglich lediglich für den Bereich des Führungskräftenachwuchses entwickelt wurde (ROSENSTIEL und NERDINGER, 2000). Konzeptionell erscheint hierbei auch die Abgrenzung zum Einstellungskonstrukt sinnvoll. Der Unterschied zwischen diesen beiden Konstrukten wird primär in ihrem Spezifitätsniveau gesehen (STENGEL, 1987). So richten sich Einstellungen auf spezifische Objekte, Personen bzw. Situationen, während Berufsorientierungen situations-, person- und objektübergreifend das berufliche Handeln beeinflussen. Anders ausgedrückt, fokussiert eine Einstellung stets auf abgrenzbare Objektklassen oder lediglich auf ein einzelnes Objekt, während Berufsorientierungen ein Bündel aus ähnlichen Einstellungen bilden und somit übergreifende Regelungskapazitäten aufweisen. Aufgrund einer Vielzahl von quer- und längsschnittlichen Untersuchungen mit unterschiedlichen Zielgruppen ist die Konstruktvalidität von Berufsorientierungen als evident zu werten, und zwar hinsichtlich der Zusammenhänge mit Werthaltungen, beruflichen Einstellungen sowie Persönlichkeitsmerkmalen und Motiven (vgl. MAIER et al., 2009).

Neben einem strukturellen Persönlichkeitsaspekt umfassen Berufsorientierungen auch einen langfristigen Zukunfts- und Zielbezug des Handelns, wie er in der Motivationspsychologie untersucht wird (BLICKLE, 1999). Dort wird angenommen, dass Motive die Anregung, Selektion und Steuerung von Handlungsweisen erklären können. Die prädiktive Validität der Berufsorientierungen konnte in einer Vielzahl von Untersuchungen an Studierenden und Führungskräften bestätigt werden. So konnte gezeigt werden, dass die Wahl der ersten Beschäftigung von Hochschulabsolventen insbesondere vor dem Hintergrund der eigenen Berufsorientierungen erfolgt (LANG VON-WINS et al., 1995). Ebenso ist eine externe Validierung des Konstruktes der Berufsorientierungen anhand der Wochenarbeitsstunden, der Anzahl der Urlaubswochen, der Dauer der beruflichen Arbeit zu Hause sowie der Anzahl von Freizeitstunden von KOLLAR und STENGEL (1990) vorgelegt worden.

Obgleich die aufgezeigten Modelle eine nach persönlichkeitsspezifischen Merkmalen potenzieller Bewerber differenzierende Betrachtung nahelegen, existieren bislang nur wenige Studien, die diese Annahmen mittels theoretisch fundierter Konstrukte empirisch überprüft haben (vgl. Abschnitt 2.5.1). An dieser Stelle kommt das Konstrukt der Berufsorientierungen zum Tragen. Dabei wird im Einzelnen angenommen, dass für karriereorientierte Bewerber vor allem die beiden Arbeitsplatzmerkmale Bezahlung und Entwicklung- und Aufstiegschancen bedeutsam sind. So konnten KOYS et al. (1989) zeigen, dass die Präferenz für eine leistungsorientierte Bezahlung mit der positiven Einschätzung der eigenen Leistung zusammenhängt. Da die Karriereorientierung positiv mit der expliziten Leistungsmotivation einhergeht, wird von einer solchen Einschätzung bei karriereorientierten Bewerbern ausgegangen. Ebenso ergab die Untersuchung von CABLE und JUDGE (1994), dass die von den potenziellen Bewerbern bevorzugte Gehaltshöhe mit ihren Persönlichkeitseigenschaften und Wertvorstellungen korreliert. So präferieren Personen mit ausgeprägten materialistischen Wertvorstellungen eher ein hohes bzw. 
überdurchschnittliches Gehalt. Demgegenüber lässt sich bei freizeitorientierten Bewerbern eine Präferenz für die Einhaltung der arbeitsvertraglich festgelegten Arbeitszeit erwarten. In diesem Zusammenhang konnten KOLLAR und STENGEL (1990) nachweisen, dass karriereorientierte und alternativ engagierte Führungskräfte eine überdurchschnittliche Wochenarbeitszeit eher in Kauf nehmen als freizeitorientierte und somit deutlich mehr Zeit in ihren Beruf investieren.

\subsection{Erklärung der Bewerbungsabsicht}

Ein wesentliches Forschungsziel dieser Arbeit besteht darin, das Zustandekommen der Bewerbungsintention anhand eines möglichst umfassenden Kausalmodells systematisch abzubilden und zu erklären. Dabei wird analog zur Einschätzung von BECKER et al. (2008) davon ausgegangen, dass die alleinige Fokussierung auf direkt messbare Phänomene, wie beispielsweise die Anzahl tatsächlich abgeschickter Bewerbungen, einen substanziellen Teil des möglichen Erkenntnisgewinns verwehren würde. Sinnvoller erscheint es dagegen, ein integratives Rahmenmodell aufzustellen, in dem sowohl psychologische als auch marketingwissenschaftliche Konstrukte als Determinanten der Bewerbungsintention berücksichtigt und hinsichtlich ihres jeweiligen Erklärungsbeitrags überprüft werden. Eine systematische Analyse der Bewerbungsintention ist deshalb so wesentlich, da der Übergang vom potenziellen zum tatsächlichen Bewerber gemäß dem Prozessmodell von MOSER und ZEMPEL (2006) die Voraussetzung für alle weiterführenden Phasen darstellt. Eine genaue Kenntnis um die eine Bewerbungsabsicht beeinflussenden Faktoren ermöglicht es den Unternehmen, ihre Strategien und Instrumente präziser auf die Zielgruppensegmente für die Personalakquisition auszurichten. Obwohl bekannt ist, dass nicht jede Bewerbungsabsicht aufgrund des moderaten Intentions-Verhaltens-Zusammenhangs auch zu einem entsprechenden Bewerbungsverhalten führt, spiegelt sich darin doch die grundsätzlich vorhandene Bewerbungsbereitschaft und das Interesse an einem Arbeitgeber wider, woraus Personalverantwortliche wertvolle Informationen für ihre Unternehmenspraxis ableiten können.

\subsubsection{Arbeitgeberattraktivität}

Wie oben ausgeführt, sind Unternehmen in ihrer Rolle als Arbeitgeber bestrebt, potenziellen Bewerbern möglichst attraktiv zu erscheinen. Vor diesem Hintergrund stellt die Arbeitgeberattraktivität ein zentrales Konstrukt innerhalb des Modells zur Erklärung der Bewerbungsabsicht dar. Allerdings liegt hierfür bislang keine einheitliche Konstruktdefinition vor. So wird die Arbeitgeberattraktivität zum einen als eine positiv-affektive Einstellung gegenüber einer Organisation definiert, die ihrerseits mit der Motivation einhergeht, mit ihr eine Beziehung zu initiieren und in sie einzutreten (AIMAN-SMITH et al., 2001). RYNES (1991) legt der Arbeitgeberattraktivität hingegen eine deutlich unspezifischere Definition zugrunde, indem er darunter die subjektive Wahrnehmung einer Organisation als einen erstrebenswerten Arbeitsort versteht. 
Ungeachtet der vorhandenen definitorischen Unterschiede ergibt sich aus den Studien auch ein gemeinsamer inhaltlicher Kern, der darin besteht, dass potenzielle Bewerber ein Unternehmen im Sinne eines Einstellungsobjektes subjektiv bewerten und anschließend positiv oder negativ darauf reagieren. Hieraus wird zugleich deutlich, dass es sich bei der Arbeitgeberattraktivität um ein Einstellungskonstrukt handelt (EAGLY und CHAIKEN, 1993). Eine weitere Möglichkeit, das Konstrukt der Arbeitgeberattraktivität näher zu spezifizieren, besteht in der Abgrenzung zum Präferenzbegriff, der in der Forschung zur Arbeitgeberwahl ebenfalls einen hohen Stellenwert einnimmt. Sowohl Attraktivität als auch Präferenz sind nicht direkt beobachtbar und werden in evaluativen bzw. präferentiellen Urteilen zum Ausdruck gebracht (JUNGERMANN et al., 2010). Der Hauptunterschied liegt hierbei darin, dass sich die Arbeitgeberattraktivität durch eine absolute Bewertung eines Unternehmens auszeichnet, während die Präferenz auf seiner relativen Beurteilung basiert.

Die teils stark abweichende begriffliche Einordnung des Attraktivitätskonstruktes geht zugleich mit unterschiedlichen Erklärungsansätzen und Operationalisierungsmethoden einher. So wird die Arbeitgeberattraktivität zum einen als eine generelle positive Einstellung gegenüber einer Organisation erfasst (z.B. HIGHHOUSE et al., 2003). Zum anderen wird sie anhand der Bereitschaft, sich für eine Anstellung bei einem Unternehmen besonders zu bemühen, gemessen (z.B. TURBAN, 2001). In marketingwissenschaftlichen Studien wird das Konstrukt der Arbeitgeberattraktivität indes teilweise mit dem Arbeitgeberimage gleichgesetzt, ohne dabei die oben erläuterten Unterschiede adäquat zu berücksichtigen (z.B. HAUTZINGER, 2009). An dieser Stelle ist es daher nicht weiter verwunderlich, dass aus den unterschiedlichen Operationalisierungsmethoden der Arbeitgeberattraktivität auch uneinheitliche Forschungsergebnisse hinsichtlich ihrer Wirkung resultieren. Es zeigt sich dabei, dass ein einheitliches und allgemein anerkanntes Verständnis des Konstruktes Arbeitgeberattraktivität bislang noch nicht vorliegt (EHRHART und ZIEGERT, 2005). Vor diesem Hintergrund wird die Arbeitgeberattraktivität gemäß den Annahmen der TOPB als Einstellung zum Verhalten aufgefasst und inhaltlich auf die spezifische Entscheidungssituation der Arbeitgeberwahl übertragen.

\subsubsection{Organisationales Prestige}

Gemäß der TOPB umfasst die subjektive Norm den wahrgenommenen sozialen Druck, der sich entweder fördernd oder hemmend auf die Ausführung eines bestimmten Verhaltens auswirkt (AJZEN, 1991). Als konstituierend für diese Modellkomponente werden die normativen Überzeugungen, die sich ihrerseits aus den Erwartungen wichtiger Bezugspersonen und der persönlichen Motivation, diesen Erwartungen auch nachkommen zu wollen, betrachtet. Allerdings deuten die metaanalytischen Befunde von ARMITAGE und CONNER (2001) darauf hin, dass der Erklärungsbeitrag der subjektiven Norm im Vergleich zu den beiden übrigen Determinanten deutlich geringer ausfällt. Die Autoren führen dies unter anderem auf eine uneinheitliche Ope- 
rationalisierung zurück. Demzufolge werden die beiden Konstrukte Einstellung zum Verhalten und wahrgenommene Verhaltenskontrolle mittels Multi-Item-Skalen erfasst, während die subjektive Norm häufig mit einer Single-Item-Skala und den damit einhergehenden methodischen Einschränkungen gemessen wird.

Betrachtet man die unterschiedlichen in Studien zur Arbeitgeberwahl verwendeten Konstrukte, so kommt das Prestigekonstrukt in seiner inhaltlichen Ausgestaltung der subjektiven Norm der TOPB sehr nahe. Diese Ähnlichkeit wurde in der bisherigen Forschung allerdings kaum registriert und entsprechend nur selten angemessen berücksichtigt. Darüber hinaus ist festzuhalten, dass das organisationale Prestige teilweise synonym mit den beiden inhaltlich durchaus verwandten Konstrukten Unternehmensimage und Reputation verwendet wird (HILDEBRANDT et al., 2008). HERRBACH und MiGNONAC (2004: 77) grenzen es inhaltlch insofern ab, als dass sie darunter ,a concept describing the way members interpret and assess their organisation's reputation based on their exposure to information about the organisation" verstehen. Beim organisationalen Prestige steht somit die subjektive Einschätzung einer Person darüber im Vordergrund, wie externe Personen ihre (potenzielle) Organisationsmitgliedschaft beurteilen (BARNETT et al., 2006). Demgegenüber beruht die Reputation auf der öffentlichen Meinung und bildet die Grundlage für die unmittelbare Bewertung eines Unternehmens durch externe Personen, zu denen beispielsweise auch potenzielle Bewerber gehören (HERRBACH und MigNONAC, 2004; HELM, 2007). Das organisationale Prestige zeichnet sich zudem durch seine motivationspsychologische Komponente aus, die sich in der gewünschten sozialen Anerkennung samt der Emotion Stolz manifestieren lässt (HIGHHOUSE et al., 2003). Schließlich unterscheidet sich das Arbeitgeberimage insoweit vom organisationalen Prestige, als dass das zuerst genannte Konstrukt eine öffentlich zugängliche Bewertungsgrundlage für das zuletzt genannte darstellt (HELM, 2007).

Das Prestige eines Unternehmens resultiert aus unterschiedlichen direkten und indirekten Kommunikationsquellen, wobei für potenzielle Bewerber die hieraus entstehende Einschätzung durch unternehmensexterne Personen von zentraler Bedeutung ist (DUTTON et al, 1994). Eine als positiv wahrgenommene Beurteilung der Organisationsmitgliedschaft durch relevante unternehmensexterne Bezugspersonen mündet für potenzielle Bewerber demzufolge in einer sozialen Stärkung ihres eigenen Selbstkonzeptes, was wiederum zu einer höheren Identifikation mit dem künftigen Arbeitgeber führt. Diese Annahme lässt sich aus entscheidungspsychologischer Perspektive weiter spezifizieren, indem hier zwischen dem sog. heißen und kalten Nutzen unterschieden wird (JUNGERMANN et al., 2010). So wird davon ausgegangen, dass Personen Konsequenzen und Situationen nicht nur abstrakt als mehr oder weniger nützlich bewerten, sondern dass die Nutzenerfahrung auch spezifische bzw. konkrete Emotionen, wie beispielsweise Freude, Stolz oder Angst, mit einschließt. Demzufolge wird der aggregierte Nutzen in einer Situation als hoch bewertet, wenn die positiven Emotionen überwiegen. Innerhalb des Prestigekonstruktes wird der Emotion Stolz eine zentrale Bedeutung beigemessen, sodass 
diese Emotion auch entsprechend bei der Operationalisierung berücksichtigt wird (CABLE UND TURBAN, 2003). Betrachtet man die Effekte des organisationalen Prestiges innerhalb der Arbeitgeberwahl genauer, so zeigt sich, dass es sich sowohl auf die Bewerbungsintention als auch auf die Arbeitgeberattraktivität auswirkt (CARMELI und FREUND, 2002; HiGHHOUSE et al., 2003). Vor diesem Hintergrund wird auch hier angenommen, dass das organisationale Prestige die Bewerbungsintention sowohl auf direktem als auch auf dem indirekten Wege, vermittelt über die wahrgenommene Arbeitgeberattraktivität, beeinflusst.

\subsubsection{Erfolgswahrscheinlichkeit der Bewerbung}

Für die Annahmen der TOPB gilt generell, dass die prädiktive Kraft der wahrgenommenen Verhaltenskontrolle umso höher ausfällt, je mehr sie der objektiven Verhaltenskontrolle entspricht (BAMBERG, 1999). Damit ist gemeint, dass Personen unter manchen Bedingungen, beispielsweise wenn ihnen sämtliche notwendigen Informationen vorliegen, Urteile nach den Regeln der Wahrscheinlichkeitstheorie abgeben könnten (JUNGERMANN et al., 2010). Wie im Abschnitt 3.7 dargestellt, trifft dies allerdings nur in sehr seltenen Fällen zu, sodass die Urteile im Normalfall intuitiv gefällt werden und dadurch häufig die Regeln der Wahrscheinlichkeitstheorie verletzen. Im Regelfall ist bei dieser Modellkomponente somit von einer deutlichen Diskrepanz zwischen der objektiven und der wahrgenommen Verhaltenskontrolle auszugehen. Hinsichtlich der Bewerbungsintention ist hierbei anzunehmen, dass die objektive Erfolgswahrscheinlichkeit substantiell von der subjektiv eingeschätzten Erfolgswahrscheinlichkeit der Bewerbung abweichen kann, da die erforderlichen Informationen einem potenziellen Bewerber i.d.R. nicht zugänglich sind. Gleichwohl konnte in der Studie von LEWANDOWSKI und LIEBIG (2004) gezeigt werden, dass die subjektiv eingeschätzte Erfolgswahrscheinlichkeit der Bewerbung ein signifikanter Prädiktor für die Bewerbungsabsicht ist. Dieser Befund könnte damit erklärt werden, dass potenzielle Bewerber anhand dieser Modellkomponente einschätzen, inwieweit sie die Bewerbungssituation selbst positiv beeinflussen können, was sich wiederum positiv auf ihre Absichtsbildung auswirkt. Die Modellkomponente wahrgenommene Verhaltenskontrolle wird innerhalb des Teilmodells zur Erklärung der Bewerbungsintention als Erfolgswahrscheinlichkeit der Bewerbung konzipiert.

\subsubsection{Alternativen auf dem Arbeitsmarkt}

Eine weitere Modellkomponente, die in den bisherigen Ansätzen nur selten berücksichtigt wurde, ist die Wahrnehmung und Einschätzung von Alternativen auf dem Arbeitsmarkt (ARNOLD et al., 2006). Die aktuelle Arbeitsmarklage wird im Kontext der TOPB als eine externe Ressource aufgefasst, die sich unmittelbar auf die wahrgenommene Verhaltenskontrolle, hier die Erfolgswahrscheinlichkeit der Bewerbung auswirkt. Demzufolge stellen RoSENSTIEL und 
NERDINGER (2000) fest, dass die Selbstselektionseffekte potenzieller Bewerber bei einer positiven Arbeitsmarktlage besonders hoch ausfallen sollten, da sie bei ihrer Arbeitgeberwahl weniger ökonomischen Restriktionen unterliegen. Eine günstige Arbeitsmarktsituation sollte sich demzufolge förderlich auf die prädiktive Kraft der proximalen Intentionsdeterminante Erfolgswahrscheinlichkeit der Bewerbung auswirken.

\subsubsection{Person-Organisation-Passung}

Ein weiteres Konstrukt, das im Rahmen der Arbeitgeberwahl eine große Aufmerksamkeit erfahren hat, ist die Person-Organisation-Passung (KRISTOF, 1996). Auch dazu liegen jedoch teils abweichende theoretische und methodische Konzeptionen vor (KRISTOF-BROWN et al, 2005). Gemäß der systematischen Differenzierung von KRISTOF (1996) wird hier auf die subjektive Person-Organisation-Passung in Form der supplementären Passung zurückgegriffen, bei der die Kongruenz zwischen organisationalen und persönlichkeitsspezifischen Merkmalen von Bewerbern im Vordergrund steht. Es wird somit als ein subjektiv empfundenes Maß an Übereinstimmung zwischen dem Arbeitgeberimage einerseits und den Werthaltungen, persönlichen beruflichen Zielen und Bewerbererwartungen andererseits aufgefasst (vgl. CARLESS, 2005). In den psychologischen Studien zur Arbeitgeberwahl konnte für das Konstrukt ein hoher Stellenwert herausgearbeitet werden. So konnte gezeigt werden, dass die direkt erhobene subjektive Person-Organisation-Passung substanziell mit der Arbeitgeberattraktivität korreliert (CHAPMAN et al., 2005). JUDGE und CABLE (1997) konnten in ihrer Untersuchung indes zeigen, dass der Zusammenhang zwischen der objektiv erfassten Person-Organisation-Passung und der Arbeitgeberattraktivität durch die subjektiv gemessene Person-Organisation-Passung vermittelt wird. Aus der längsschnittlichen Untersuchung von CARLESS (2005) konnte zudem die Richtung des kausalen Zusammenhangs nachgewiesen werden, wonach die Person-Organisation-Passung die Arbeitgeberattraktivität beeinflusst. Vor diesem Hintergrund wird das Grundmodell der TOPB zur Vorhersage der Bewerbungsabsicht um die proximale Determinante Person-OrganisationPassung erweitert, deren Einfluss gleichzeitig zumindest partiell über die wahrgenommene Arbeitgeberattraktivität vermittelt wird.

\subsubsection{Branchenreputation}

Die Aufnahme branchenspezifischer Konstrukte in das Kausalmodell setzt eine vorherige Auseinandersetzung mit dem allgemeinen Branchenbegriff voraus. Generell gilt, „dass die Branche eine dem einzelnen Unternehmen hierarchisch übergeordnete Einheit ist" (HAUTZINGER, 2009: 28). BURMANN und SCHAEFER (2005: 17) spezifizieren den Branchenbegriff als „eine Gruppe von Unternehmen, die aus Sicht eines Individuums dieselben Kundengruppen mit denselben 
Technologien zur Erfüllung derselben Kundenfunktionen bedienen“. Eine solche Definition verdeutlicht zugleich, dass die Wahrnehmung einer Branche von dem zugrunde liegenden Wissen abhängig ist, da nicht alle Personen über die gleichen Kenntnisse in den oben genannten Bereichen verfügen (SCHAEFER, 2006). Nach der hier vorgelegten Definition ist es bei der Branchenzuordnung somit erforderlich, den gewählten Differenzierungsgrad an das Wissen der relevanten Zielgruppe anzupassen. Dabei wird zugleich angenommen, dass die Ernährungsindustrie einen für diese Untersuchung hinreichenden Differenzierungsgrad aufweist, sodass potenzielle Bewerber diese Branche adäquat eingrenzen können. Diese Annahme wird zugleich durch die Untersuchung von SÜSS (1996) empirisch gestützt, in der die Lebensmittelindustrie als eigenständige Branche hinsichtlich ihrer Attraktivität zu bewerten war. Darüber hinaus weisen auch praxisorientierte Studien darauf hin, dass die Ernährungsindustrie über den erforderlichen Spezifikationsgrad verfügt (z.B. GPRA, 2011). Zur definitorischen Eingrenzung ist hierbei ferner die Branchenerosion zu erwähnen, worunter die gleichzeitige Zugehörigkeit eines Unternehmens zu mehreren Branchen verstanden wird (SCHAEFER, 2006). Dieses Phänomen lässt sich jedoch insoweit auffangen, als dass es in dieser Untersuchung nicht um eine objektive Zuordnung von Unternehmen zu einer Branche geht, sondern vielmehr um die subjektive Vorstellung, die ein potenzieller Bewerber gegenüber der Ernährungsindustrie hat. Fernerhin ist im Zusammenhang mit der Branchenreputation zu beachten, dass eine Reputation zwar insgesamt positiv ausfallen kann, einzelne Branchenzweige dabei aber durchaus über deutlich schlechtere Reputationswerte verfügen können. So stellen ALBERSMEIER und SPILLER (2009) heraus, dass die Lebensmittelindustrie im Ganzen zwar als glaubwürdig gesehen wird, einzelne Subbranchen, wie beispielsweise die Fleischwirtschaft, aber gleichzeitig einer deutlichen Kritik unterzogen werden. Hieraus resultiert zumindest für diese Akteure ein deutlich schlechteres gesellschaftliches Ansehen.

Wie bedeutend der Einfluss einer intakten Unternehmensreputation auf den Rekrutierungserfolg ist, konnte bereits in einigen Untersuchungen empirisch nachgewiesen werden (z.B. WILLIAMSON et al., 2010). So konnten CABLE und TURBAN (2003) in ihrer Studie zeigen, dass eine gute Unternehmensreputation in einer höheren Anzahl von Bewerbungen resultiert. Zudem konnten die Autoren zeigen, dass die Unternehmensreputation das Ausmaß des antizipierten Stolzes beeinflusst, der aus dem Organisationseintritt eines potenziellen Bewerbers resultieren würde. Infolgedessen wird hier angenommen, dass sich die Reputation als distale Variable auf die proximalen Determinanten der Bewerbungsabsicht auswirkt. Allerdings wird in dieser Untersuchung nicht auf die Reputation einzelner Unternehmen zurückgegriffen, da diese aufgrund ihres überwiegend mittelständischen Charakters häufig nicht über einen eigenen Ruf verfügen dürften (SCHMIDTKE, 2001). Infolgedessen wird an dieser Stelle die nächsthöhere Ebene zur Verankerung einer Reputation, nämlich die Branchenebene gewählt. So ist gemäß dem Signaling-Ansatz gerade bei klein- und mittelständischen Unternehmen davon auszugehen, dass die Branchenreputation als wesentliche Stellvertreter-Information zur Beurteilung eines einzelnen 
Unternehmens fungiert (BURMANN und SCHAEFER, 2005). Ihr Wirkungsprinzip ist hierbei darin zu sehen, dass sie die Entscheidungsunsicherheit potenzieller Bewerbern zu reduzieren vermag (EISENEGGER und KÜNSTLE, 2003). Demgemäß konnten ALBERSMEIER und SPILLER (2009) zeigen, dass die Reputation der Fleischwirtschaft die Attraktivität eines Arbeitgebers beeinflusst. Daneben konnte auch HAUTZINGER (2009) in seiner Studie nachweisen, dass die Branchenreputation mit ihrer jeweiligen Arbeitgeberattraktivität und der Bewerbungsbereitschaft in dieser Branche zusammenhängt.

Insgesamt wurde die Branchenreputation in den bisherigen wissenschaftlichen Veröffentlichungen zur Arbeitgeberwahl nur selten berücksichtigt. Eine gewichtige Ursache hierfür könnte zugleich darin liegen, dass die Operationalisierung des Reputationskonstruktes bislang sehr uneinheitlich erfolgt und auf abweichenden Begriffsdefinitionen bzw. Messmodellen basiert (siehe für einen Überblick: ALBERSMEIER und SPILLER, 2009). Ein weiterer Grund ist darin zu sehen, dass die Relevanz dieses Konstruktes innerhalb der Arbeitgeberwahl teilweise ad hoc angenommen wird, ohne anhand theoretischer Ansätze hergeleitet worden zu sein.

\subsubsection{Branchenwissen}

Aus psychologischer Sicht stellt das Erfahrungswissen einen wesentlichen Bestandteil des Branchenwissens dar. Diese theoretische Konzeption basiert auf dem Befund von FAZIO und ZANNA (1981), dem zufolge Einstellungen, denen direkte Erfahrungen mit dem Einstellungsobjekt zugrunde liegen, höher mit Intentionen korrelieren als Einstellungen mit indirekten Erfahrungen und sich somit als prädiktiver für nachfolgendes Verhalten erweisen. Bei diesen Einstellungsobjekten kann es sich um Personen, Situationen oder Gegenstände handeln. JONAS und DOLL (1996) führen dieses Ergebnis zum einen darauf zurück, dass die entsprechenden Einstellungen besser im Gedächtnis verfügbar sind und bei der Intentionsbildung und Verhaltensrealisierung daher leichter bzw. schneller abgerufen werden können. Überdies gehen sie hierbei von einer größeren zeitlichen Stabilität aus, da Einstellungen mittels einer höheren Anzahl von verhaltensrelevanten Kognitionen länger aufrechterhalten werden. DOLL und AJZEN (1992) sehen eine wesentliche Bedingung für die Zugänglichkeit einer Einstellung in der Häufigkeit, mit der Einstellungsobjekte in der Vergangenheit mit einer bestimmten Bewertung verknüpft wurden. $\mathrm{Zu}$ diesen Bewertungen zählen vorangegangene affektive Reaktionen auf das Einstellungsobjekt oder kognitive Einschätzungen des Nutzens von entsprechenden Verhaltenskonsequenzen.

Die Berücksichtigung des Branchenwissens bei der Vorhersage der Bewerbungsintention kann ferner dadurch erklärt werden, dass es das Entscheidungsverhalten wesentlich beeinflusst, indem es unsicherheitsreduzierend wirkt und somit die Relevanz von anderweitig verfügbaren Stellvertreter-Informationen oder Heuristiken vermindert (TROMMSDORFF, 2009). Bei die- 
sem Konstrukt geht es zugleich nicht um objektives Wissen, das produktspezifische und faktenbasierte Informationen enthält, sondern um subjektives Wissen, das die Alternativen- und Merkmalseinschätzung bedingt (CABLE und TURBAN, 2003; HAUTZINGER, 2009). Gemäß dem unsicherheitsreduzierenden Ansatz wird davon ausgegangen, dass das Branchenwissen die Wirkbeziehung der Branchenreputation auf die proximalen Determinanten der Bewerbungsabsicht vermittelt.

\subsection{Zusammenfassende Darstellung der Untersuchungsmodelle und Hypothesen}

Anhand der obigen Ausführungen werden im Folgenden die daraus resultierenden forschungsleitenden Hypothesen formuliert und mithilfe eines dazugehörigen Untersuchungsmodells graphisch veranschaulicht.

Das erste Teilmodell beschäftigt sich mit der Analyse des Informationsverhaltens potenzieller Bewerber. Hierbei wird überprüft, ob und inwieweit die ausgewählten Kommunikationsmaßnahmen mit den soziodemographischen und persönlichkeitsspezifischen Merkmalen potenzieller Bewerber zusammenhängen. Die Analyse dieser Zusammenhänge hat zugleich einen eher explorativen Forschungscharakter, da bislang nur wenige theoretische Annahmen und empirische Studien vorliegen, aus denen strenge Vorannahmen über die Art der Zusammenhänge ableitbar wären.

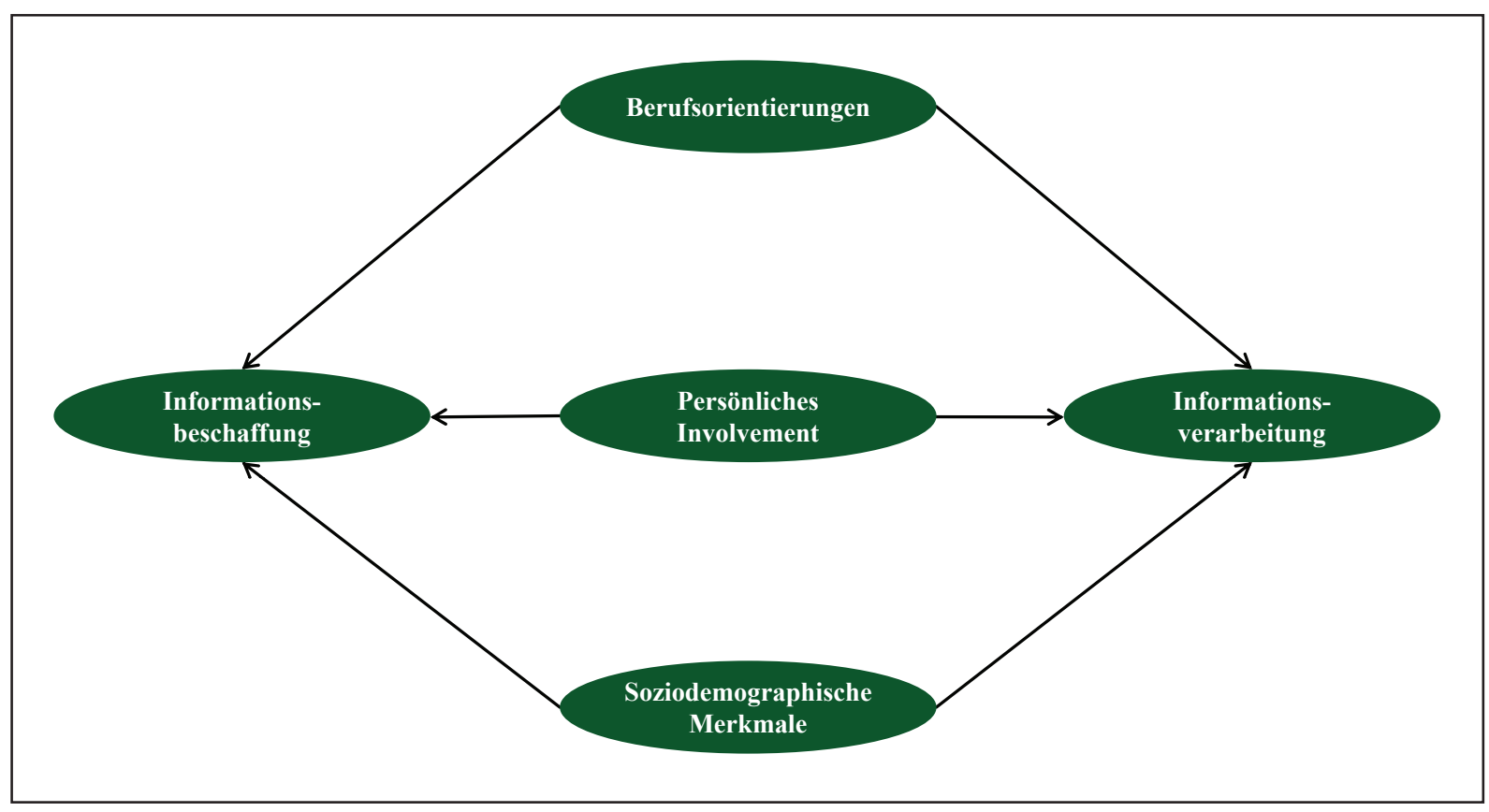

Abb. 4-1: Postulierte Zusammenhänge im ersten Teilmodell (Eigene Darstellung, 2011) 
H1: Es wird angenommen, dass der Bekanntheitsgrad einzelner Kommunikationsmaßnahmen mit den soziodemographischen und persönlichkeitsspezifischen Variablen zusammenhängt.

H2: Es wird angenommen, dass die Nutzungsintensität einzelner Kommunikationsmaßnahmen mit den soziodemographischen und persönlichkeitsspezifischen Variablen zusammenhängt.

H3: Es wird angenommen, dass die Bewertung einzelner Kommunikationsmaßnahmen mit den soziodemographischen und persönlichkeitsspezifischen Variablen zusammenhängt.

H4: Es wird angenommen, dass das Präferenzmaß einzelner Kommunikationsmaßnahmen mit den soziodemographischen Variablen zusammenhängt.

Zahlreiche wissenschaftliche und praxisorientierte Studien haben sich mit der Bedeutung von Arbeitsplatzmerkmalen befasst (vgl. Abschnitt 2.5.1). Dennoch ist die zugrunde liegende Forschungslage sowohl aus theoretischen als auch methodischen Gründen bislang als kritisch zu werten. Insbesondere die unmittelbare Erhebung von Bedeutsamkeitsurteilen hat sich in diesem Kontext als wenig geeignet erwiesen. Stattdessen wird im zweiten Teilmodell ein dekompositionelles Präferenzmessverfahren angewendet, mit dessen Hilfe die mit direkten Befragungen verbundenen validitätsmindernden Einschränkungen zumindest teilweise umgangen werden sollen.

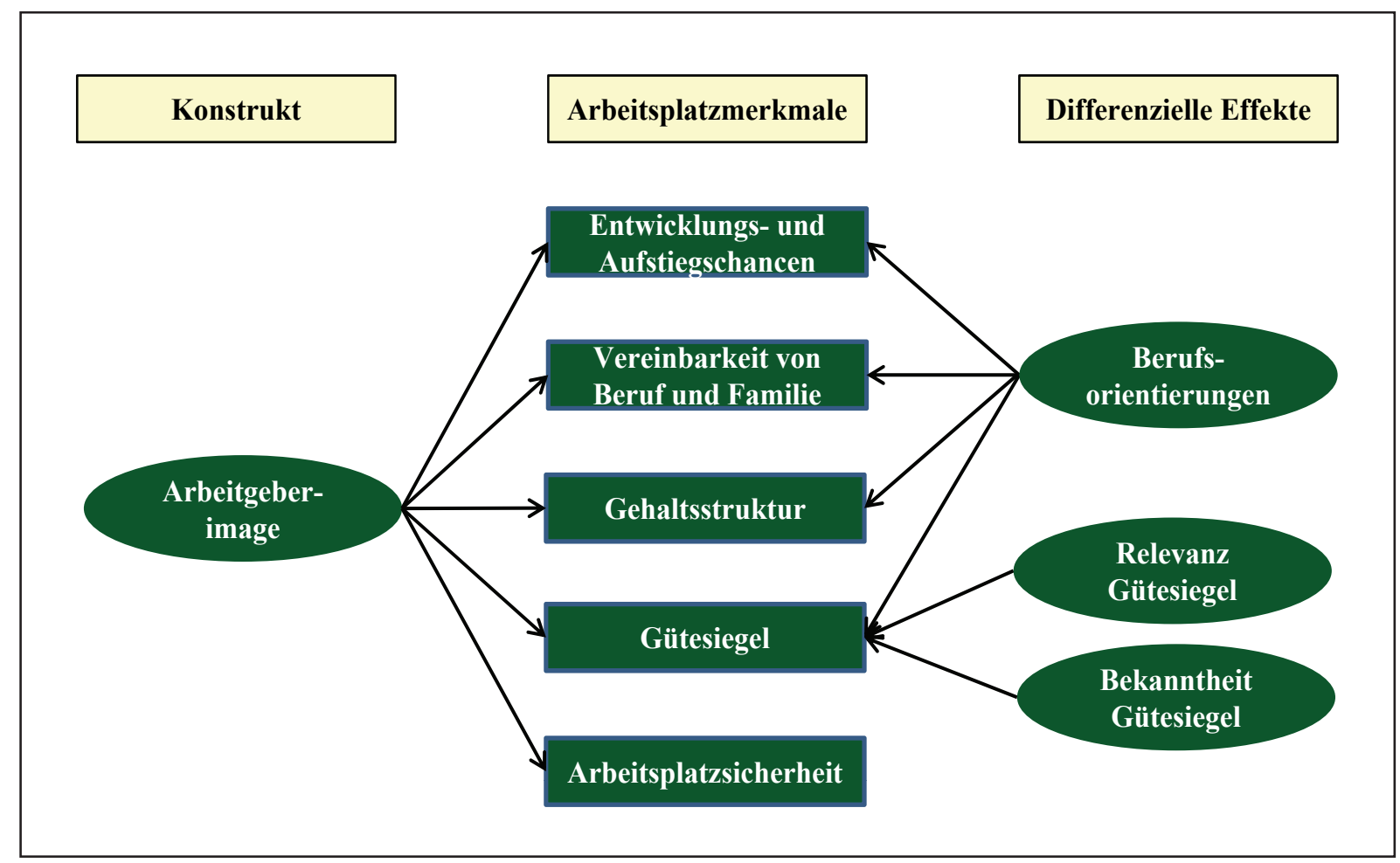

Abb. 4-2: Postulierte Zusammenhänge im zweiten Teilmodell (Eigene Darstellung, 2011) 
H5: Die Entwicklungs- und Aufstiegschancen führen zu einer höheren Wahlwahrscheinlichkeit des Stellenangebotes.

H6: Die Vereinbarkeit von Beruf und Familie bzw. Privatleben führt zu einer höheren Wahlwahrscheinlichkeit des Stellenangebotes.

H7: Die leistungsorientierte Vergütung führt zu einer höheren Wahlwahrscheinlichkeit des Stellenangebotes.

H8: Die Arbeitsplatzsicherheit führt zu einer höheren Wahlwahrscheinlichkeit des Stellenangebotes.

H9: Das Gütesiegel ,audit berufundfamilie“ führt zu einer höheren Wahlwahrscheinlichkeit des Stellenangebotes.

H10: Das Gütesiegel „Top Job“ führt zu einer höheren Wahlwahrscheinlichkeit des Stellenangebotes.

H11: Die Interaktion zwischen den Entwicklungs- und Aufstiegschancen und der Karriereorientierung führt zu einer höheren Wahlwahrscheinlichkeit des Stellenangebotes.

H12: Die Interaktion zwischen der Vereinbarkeit von Beruf und Familie bzw. Privatleben und der Freizeitorientierung führt $\mathrm{zu}$ einer höheren Wahlwahrscheinlichkeit des Stellenangebotes.

H13: Die Interaktion zwischen der leistungsorientierten Vergütung und der Karriereorientierung führt zu einer höheren Wahlwahrscheinlichkeit des Stellenangebotes.

H14: Die Interaktion zwischen dem Gütesiegel ,audit berufundfamilie“ und der Freizeitorientierung führt zu einer höheren Wahlwahrscheinlichkeit des Stellenangebotes.

H15: Die Interaktion zwischen dem Gütesiegel „Top Job“ und der Karriereorientierung führt zu einer höheren Wahlwahrscheinlichkeit des Stellenangebotes.

H16: Die Interaktion zwischen Gütesiegeln und ihrem jeweiligen Bekanntheitsgrad führt zu einer höheren Wahlwahrscheinlichkeit des Stellenangebotes.

H17: Die Interaktion zwischen Gütesiegeln und ihrer generellen Relevanz führt zu einer höheren Wahlwahrscheinlichkeit des Stellenangebotes. 
Im Fokus des dritten Teilmodells steht die Forschungsfrage, anhand welcher Faktoren die Bewerbungsintention vorhergesagt werden kann. Als Rahmenmodell zur Erklärung und Vorhersage der Bewerbungsintention wird die TOPB herangezogen, wobei die darin spezifizierten Prädiktoren um weitere theoretisch hergeleitete Determinanten ergänzt werden. Diese werden inhaltlich auf die zugrunde liegende Entscheidungssituation der Arbeitgeberwahl angepasst, woraus die Variablen Arbeitgeberattraktivität, organisationales Prestige sowie Erfolgswahrscheinlichkeit der Bewerbung resultieren. Daneben wird die Person-Organisation-Passung als eine weitere proximale Determinante der Bewerbungsabsicht in das Teilmodell integriert. Als distale Variablen der Bewerbungsintention fungieren der Branchenruf, das Branchenwissen sowie die Alternativen am Arbeitsmarkt. Neben ihrem direkten Einfluss werden für die beiden Variablen Branchenwissen und Arbeitgeberattraktivität mediierende Effekte überprüft.

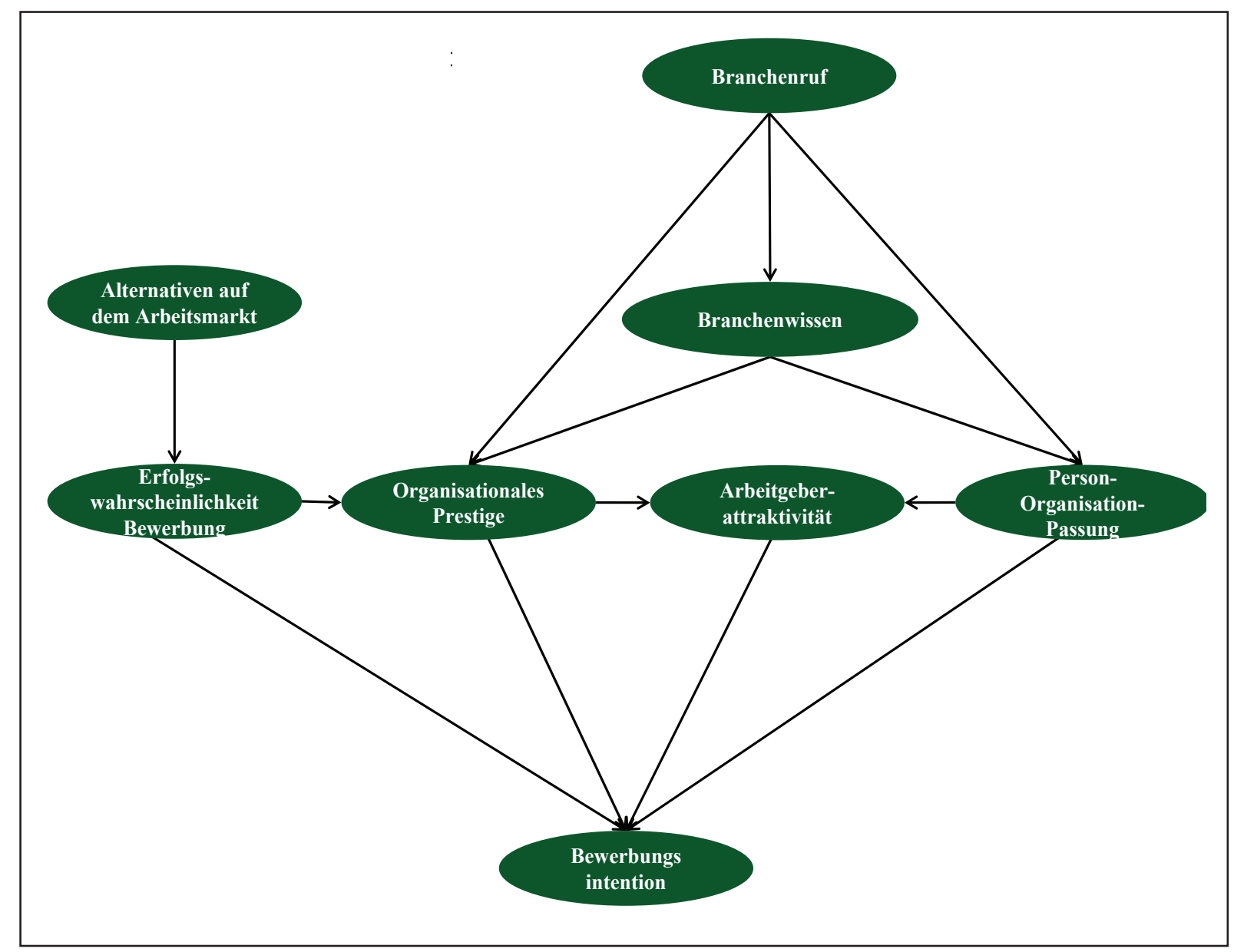

Abb. 4-3: Postulierte Zusammenhänge im dritten Teilmodell (Eigene Darstellung, 2011)

H18: Es besteht ein positiver Zusammenhang zwischen dem Branchenruf und der PersonOrganisation-Passung.

H19: Es besteht ein positiver Zusammenhang zwischen dem Branchenruf und dem organisationalen Prestige. 
H20: Es besteht ein positiver Zusammenhang zwischen den wahrgenommenen beruflichen Chancen auf dem Arbeitsmarkt und der subjektiv eingeschätzten Erfolgswahrscheinlichkeit der Bewerbung.

H21: Es besteht ein positiver Zusammenhang zwischen der Person-Organisation-Passung und der Bewerbungsabsicht.

H22: Es besteht ein positiver Zusammenhang zwischen dem organisationalen Prestige und der Bewerbungsabsicht.

H23: Es besteht ein positiver Zusammenhang zwischen der wahrgenommenen Arbeitgeberattraktivität und der Bewerbungsabsicht.

H24: Es besteht ein positiver Zusammenhang zwischen der subjektiv eingeschätzten Erfolgswahrscheinlichkeit der Bewerbung und der Bewerbungsabsicht.

H25: Die wahrgenommene Arbeitgeberattraktivität mediiert die positive Beziehung zwischen der Person-Organisation-Passung und der Bewerbungsabsicht (Mediatorhypothese).

H26: Die wahrgenommene Arbeitgeberattraktivität mediiert die positive Beziehung zwischen dem organisationalen Prestige und der Bewerbungsabsicht (Mediatorhypothese).

H27: Das Branchenwissen mediiert die positive Beziehung zwischen dem Branchenruf und der Person-Organisation-Passung (Mediatorhypothese).

H28: Das Branchenwissen mediiert die positive Beziehung zwischen dem Branchenruf und dem organisationalen Prestige (Mediatorhypothese). 


\section{KAPITEL 5}

\section{Methodisches Vorgehen}

Im Folgenden wird die methodische Herangehensweise dargestellt, mit der die im Abschnitt 4.4 formulierten Hypothesen überprüft wurden. Dabei wird zunächst auf das experimentelle Design der Discrete-Choice-Analyse und die Strukturgleichungsmodellierung eingegangen. Hierbei wird auch die Operationalisierung der verwendeten Konstrukte detailliert erläutert. Schließlich wird im Kapitel 5.6 die methodische Durchführung der empirischen Untersuchung beschrieben.

\subsection{Methodenwahl für die Messung des zweiten Teilmodells}

In den bisherigen empirischen Studien zur Arbeitgeberwahl wurden die Präferenzen hinsichtlich bestimmter Arbeitsplatzmerkmale überwiegend direkt ermittelt, wobei die Wichtigkeit der betrachteten Arbeitsplatzmerkmale entweder anhand einer ordinalen Rangfolge oder einzeln mittels einer Ratingskala abgebildet wurde (vgl. Abschnitt 2.5.1). Einige Studien basieren dabei auf einem erwartungswerttheoretischen Modell, bei dem die Befragten anhand von Ratingskalen zunächst jedem Arbeitsplatzmerkmal ein Präferenzgewicht vergeben und anschließend die wahrgenommene Ausprägung dieses Arbeitsplatzmerkmals bei einem bestimmten Arbeitgeber beurteilen. Der Gesamtnutzenwert eines Arbeitgebers wird über eine gewichtet-additive Aggregationsregel ermittelt, indem die Bewertungen der einzelnen Ausprägungen der Arbeitsplatzmerkmale mit den dazugehörigen Gewichten multipliziert und über alle Arbeitsplatzmerkmale addiert werden. Solche Verfahren der multiattributiven Präferenzmessung, in denen der Gesamtnutzen eines Merkmals anhand direkter Präferenzabfragen nach einzelnen Eigenschaften erfolgt, werden als kompositionelle Methoden bezeichnet (SATTLER, 2006).

Vereinzelt wurde die Wichtigkeitsbeurteilung von Arbeitsplatzmerkmalen mittels sog. Imagedifferenziale erfasst, indem zum einen die Eigenschaftsausprägungen bei einem konkreten Arbeitgeber mit den Idealvorstellungen von Befragten verglichen wurden (vgl. z.B. LEWANDOWSKI und LIEBIG, 2004). Hierbei werden unter der Annahme eines einfachen Modells die Differenzen bei den jeweiligen Merkmalsausprägungen zu einem individuellen Gesamtnutzenwert zusammengesetzt, wobei der Gesamtnutzen umso größer ist, je geringer die resultierende 
Differenz ist. Aus den so berechneten Distanzen werden entsprechende Hinweise auf die Arbeitgeberattraktivität eines Unternehmens abgeleitet.

Die direkte Ermittlung von Bewerberpräferenzen ist jedoch mit mehreren validitätsmindernden Aspekten behaftet. So unterliegt es aufgrund der direkten Befragung einer starken Tendenz zur sozialen Erwünschtheit, wodurch es zu deutlichen Verzerrungen in den Ergebnissen, insbesondere beim Arbeitsplatzmerkmal Bezahlung, kommen kann (ARNOLD und FELDMAN, 1981). Ferner sind Ratingskalen generell mit einer Reihe von Fehlerquellen, wie der sog. Tendenz zur Mitte oder dem Halo-Effekt, behaftet, was sich wiederum ungünstig auf die Reliabilität des zu messenden Konstruktes auswirkt (BORTZ und DöRING, 2009). Hinzu kommt, dass die Art, wie Wichtigkeitsurteile gefällt werden, nicht dem Vorgehen in der Realität entspricht, da potenzielle Bewerber das Arbeitgeberimage eines Unternehmens eher global, d.h. in seiner Gesamtheit wahrnehmen und bewerten (VON WALTER et al., 2009).

Um diese methodische Problematik aufzulösen, wurde hier auf einen sog. dekompositionellen Ansatz, genauer die Discrete-Choice-Analyse (DCA), zurückgegriffen. Dieser methodische Ansatz der Präferenzmessung ist generell dadurch gekennzeichnet, dass aus der Gesamtbewertung eines Produktes mithilfe statistischer Verfahren die Wichtigkeit einzelner Eigenschaften abgeleitet wird (HIMME, 2009). Grundlegende Voraussetzung liegt wie bei den kompositionellen Verfahren auch hier darin, dass die zu beurteilenden Produkte multiattributiv beschaffen sind, d.h. aus mehreren Produkteigenschaften bestehen (BACKHAUS et al., 2011). Dekompositionelle Verfahren kamen in Studien zur Arbeitgeberwahl bislang nur vereinzelt zum Einsatz. Dazu gehörten im Einzelnen die traditionelle Conjoint-Analyse und die Policy-Capturing Methode, die im deutschsprachigen Raum eher unter der Bezeichnung Vignettenanalyse oder faktorieller Survey bekannt ist (vgl. z.B. WiLTINGER, 1997; BACKHAUS et al., 2002; GROBE, 2003; LiS, 2010).

Obgleich alle drei Verfahren, die traditionelle Conjoint-Analyse (TCA), die Vignettenanalyse bzw. die Policy-Capturing Methode und die DCA, methodisch eng verwandt sind, bestehen zwischen ihnen auch grundlegende Unterschiede. So ist der wichtigste Unterschied darin zu sehen, dass bei der TCA die Präferenzen bezüglich der betrachteten Objekte anhand von Rankings (ordinal) oder Ratings (metrisch) abgefragt werden, während die Vignettenanalyse ausschließlich Ratings zulässt (KLEIN, 2006). Demgegenüber beruht die DCA auf diskreten Auswahlentscheidungen mit einem daraus resultierenden nominalen Skalenniveau der abhängigen Variablen (TEMME, 2009). Gemäß der Nutzenmaximierungsannahme der Zufallsnutzentheorie wird hierbei angenommen, dass sich die Befragten nutzenmaximierend verhalten, sodass aus ihrem Entscheidungsverhalten zugleich Rückschlüsse auf die Nutzenbeiträge der zugrunde liegenden Eigenschaften und ihrer jeweiligen Ausprägungen abzuleiten sind (LOUVIERE et al., 2000). Hieraus ergeben sich weitere Abgrenzungsaspekte, die hier in Form einer Synopse veranschaulicht werden. 


\begin{tabular}{|c|c|c|c|}
\hline Kriterium & TCA & DCA & $\begin{array}{l}\text { Policy-Capturing/ } \\
\text { Vignettenanalyse }\end{array}$ \\
\hline Erhebung & $\begin{array}{l}\text { Präferenzurteile werden } \\
\text { erfragt anhand von } \\
\text { - Rankings } \\
\text { - Ratings }\end{array}$ & $\begin{array}{l}\text { Auswahlentscheidungen } \\
\text { werden erfragt oder } \\
\text { beobachtet }\end{array}$ & $\begin{array}{l}\text { Urteile werden erfragt } \\
\text { anhand von } \\
\text { - Ratings }\end{array}$ \\
\hline $\begin{array}{l}\text { Anzahl an } \\
\text { Attributen }\end{array}$ & $\begin{array}{l}\text { höhere Anzahl in Verfah- } \\
\text { rensvarianten (ACA) } \\
\text { möglich }\end{array}$ & begrenzt & begrenzt \\
\hline Skalenniveau AV & ordinal, metrisch & nominal & metrisch \\
\hline None-Option & nicht möglich & möglich & nicht möglich \\
\hline Analyse & $\begin{array}{l}\text { Regression } \\
\text { (Kleinstquadrate) } \\
\text { - ordinal } \\
\text { - metrisch }\end{array}$ & $\begin{array}{l}\text { Maximum Likelihood, } \\
\text { iterative Optimierung }\end{array}$ & $\begin{array}{l}\text { Regression } \\
\text { (Kleinstquadrate) } \\
\text { - metrisch }\end{array}$ \\
\hline Ergebnisse & $\begin{array}{l}\text { Teilnutzenwerte (indivi- } \\
\text { duell und aggregiert) } \\
\text { Wichtigkeiten }\end{array}$ & $\begin{array}{l}\text { Teilnutzenwerte (aggre- } \\
\text { giert), Wichtigkeiten, } \\
\text { Auswahlwahrscheinlich- } \\
\text { keiten }\end{array}$ & $\begin{array}{l}\text { Regressionskoeffizienten } \\
\text { (aggregiert) der Eigen- } \\
\text { schaftsausprägungen }\end{array}$ \\
\hline Externe Validierung & nicht möglich & $\begin{array}{l}\text { möglich über Vergleich } \\
\text { mit realem Wahlverhalten }\end{array}$ & nicht möglich \\
\hline
\end{tabular}

Tab. 5-1: Unterschiede zwischen der TCA, der DCA und der Policy Capturing bzw. Vignettenanalyse

(Eigene Darstellung, 2012, in Anlehnung an KARREN und BARRINGER, 2002; BROCKE, 2006; STEINER und ATZMÜLLER, 2006)

Aus dem oben dargestellten Vergleich der drei Ansätze lässt sich die Anwendung der DCA auf die Analyse der Arbeitgeberwahl nun eingehender begründen. So wird in dieser Arbeit der Frage nachgegangen, ob und inwieweit die Wahlentscheidung potenzieller Bewerber für ein bestimmtes Stellenangebot (= Produkt) anhand seiner Arbeitsplatzmerkmale (= Attribute) erklärt werden kann. Aus den beiden alternativen Ansätzen, der TCA und der Policy-Capturing-Methode, ließe sich diese Forschungsfrage grundsätzlich nicht eindeutig beantworten, da es sich bei der Wahlentscheidung um eine diskrete Variable handelt und klassische Regressionsansätze an dieser Stelle zu falschen Ergebnissen führen (TEMME, 2009). Der Einsatz der DCA wird zudem aufgrund der getroffenen theoretischen Annahmen als geeignet betrachtet. So wird hier angenommen, dass das Arbeitgeberimage vor allem die anfängliche Entscheidung, eine Bewerbung überhaupt in Erwägung zu ziehen, beeinflusst. Es handelt sich hierbei also um die Auswahl eines Stellenangebotes anhand der mit ihm einhergehenden Arbeitsplatzmerkmale. Ein weiterer entscheidender Vorteil ist ferner darin zu sehen, dass die DCA auf einem wahrscheinlichkeitstheoretischen Ansatz basiert, der die subjektive Nutzenbewertung und das Auswahlverhalten der Befragten theoretisch und methodisch miteinander verbindet (BROCKE, 2006). Demzufolge ist die DCA im Unterschied zur Vignettenanalyse unmittelbarer auf die Erhebung von Entschei- 
dungen zugeschnitten und stärker mit theoretischen und statistischen Entscheidungsmodellen verknüpft (AUSPURG und LIEBE, 2011).

Auch der methodische Vergleich stützt den Einsatz der DCA bei dieser Fragestellung. So birgt zum einen die obligatorische Verwendung von Ratingskalen innerhalb der Policy-Capturing Methode den Nachteil, dass die Präferenzstärke auch dann angegeben werden muss, wenn die Befragten dies nicht leisten können (BORG und STAUFENBIEL, 2007). Dieses Vorgehen würde jedoch zu einer deutlichen Verringerung der zugrunde liegenden Reliabilität führen. Zum anderen ist es aufgrund der postulierten differenziellen Bedeutsamkeitseinschätzungen einzelner Arbeitsplatzmerkmale unabdingbar, individuelle Berufsorientierungen als exogene Variablen in das Discrete-Choice-Modell aufzunehmen, was im Rahmen der TCA allerdings nicht möglich wäre (ENNEKING, 2003). Hinzu kommt, dass die Datenerhebung bei der DCA insgesamt deutlich realitätsnäher ist als bei der TCA oder der Policy-Capturing Methode, wodurch tendenziell eine höhere Validität zu erwarten ist (BACKHAUS et al., 2011). So konnte in empirischen Validitätsvergleichstests zwar gezeigt werden, dass die DCA kompositionellen Verfahren grundsätzlich überlegen ist. Bei einem Vergleich mit der TCA wurden allerdings nur marginale Vorteile für die DCA festgestellt, sodass derzeit noch nicht von einer generellen Überlegenheit der DCA ausgegangen werden kann (VÖLCKNER et al., 2008).

Aus praxisorientierter Perspektive erweist sich die DCA deshalb als besonders geeignet, da sie dem eigentlichen Unternehmensinteresse nachkommt, das Arbeitgeberwahlverhalten potenzieller Bewerber besser zu verstehen und gegebenenfalls zu prognostizieren. So wird es mit dem Verfahren möglich, die ermittelten Bewerberpräferenzen als wesentliche Richtlinien für eine zielgruppenspezifische Gestaltung der Leistungs- und Gehaltspolitik hinsichtlich des anzubietenden Arbeitsplatzes zu nutzen (SIMON et al., 1995). Es wird den Arbeitgebern somit erleichtert, potenziellen Bewerbern gezielt solche Ausprägungen von Arbeitsplatzmerkmalen anzubieten, die ihre Bewerbungsbereitschaft erhöhen.

Wenngleich für den Einsatz der DCA mehrere wesentliche Vorteile aufgezeigt werden konnten, ist das Vorgehen auch mit einigen methodischen Problemen behaftet, die es zu beachten gilt. So handelt es sich bei den Wahlaufgaben um hypothetische statt realer Entscheidungen zwischen den einzelnen Stellenangeboten, woraus keine Aussagen hinsichtlich der externen Validität des Discrete-Choice-Modells abgeleitet werden können (AUSPURG und LIEBE, 2011). Darüber hinaus erlaubt das methodische Vorgehen keine Verallgemeinerung, die über die verwendeten Abstufungen bei den Arbeitsplatzmerkmalen hinausgeht (VÖLCKNER et al., 2008). Dieser Nachteil lässt sich zugleich insoweit entschärfen, als dass die im Erhebungsdesign berücksichtigten Ausprägungen aufgrund ihres starken Verbreitungsgrades sehr realitätsnah erscheinen und somit eine für diese Forschungsfrage ausreichende Repräsentativität bieten. Ebenso wurde die Entscheidungsrelevanz der berücksichtigten Arbeitsplatzmerkmale und ihrer Ausprägungen sichergestellt, indem ausschließlich solche Eigenschaften zum Einsatz kamen, die in den bisherigen 
Studien von den Befragten als besonders wichtig beurteilt wurden. Ein weiterer kritischer Aspekt ist in der vorgenommenen Set-Bildung zu sehen. So lässt sich nicht gänzlich ausschließen, dass die jeweils zur Bewertung vorgelegten Wahlaufgaben einen spezifischen Entscheidungskontext schaffen und somit einen Einfluss auf die Präferenzbildung haben (KLEIN, 2006). Schließlich weisen diskrete Auswahlentscheidungen im Vergleich zu den anderen Präferenzmessverfahren grundsätzlich einen geringeren Informationsgehalt auf, da die Präferenzstärke bei einer Alternative nicht erhoben wird (HIMME, 2009). In der Bilanzierung der vorteilhaften und problematischen Aspekte dieses Verfahrens wird insgesamt deutlich, dass die Anwendung der DCA auf zugrunde liegende Fragestellung sowohl aus der theoretisch-methodischen als auch aus der anwendungsorientierten Perspektive besonders geeignet ist. Die folgenden Ausführungen behandeln deshalb ausschließlich die DCA.

Eine weitere Differenzierungsebene der hier gewählten Methodik betrifft die Erhebungsart. Hierbei kann zwischen einem sog. revealed-preference-Ansatz, der auf direkt beobachtbaren Daten beruht, und einem sog. stated-preference-Ansatz, in dem Befragungen verwendet werden, unterschieden werden (SATTLER, 2006). Bei dieser Untersuchung wurde der zweitgenannte Ansatz verwendet, da die Bewerbungsentscheidung mittels eines experimentellen Designs analysiert wurde und nicht auf die Erfassung tatsächlicher abgeschickter Bewerbungen abzielte (vgl. Kap. 4.3).

\subsection{Experimentelles Design der Discrete-Choice-Analyse}

Eine DCA umfasst, wie jede Form der Conjoint-Analyse, ein Erhebungsdesign und ein Analyseverfahren, die sich wiederum in weitere Teilschritte unterteilen lassen.

\section{Design}

Untersuchungsobjekt: Gestaltung der Attribute und ihrer Ausprägungen

Untersuchungsobjekt: Gestaltung der Auswahlsituationen

\section{Durchführung}

Erhebungsart

Art und Größe der Stichprobe

Modellspezifikation und Datenauswertung

Spezifikation des Nutzen- und Auswahlmodells

Schätzung der Nutzenfunktion

Interpretation der Nutzenparameter

Abb. 5-1: Vorgehen bei der DCA (Eigene Darstellung, 2012, in Anlehnung an HIMME, 2009; BACKHAUS et al., 2011) 
Demzufolge wurden im ersten Schritt alle für das Arbeitgeberimage relevanten Arbeitsplatzmerkmale und ihre dazugehörigen Ausprägungen definiert. Das methodische Ziel bestand bei diesem Teilschritt vor allem darin, die aus Bewerbersicht wichtigsten Arbeitsplatzmerkmale im experimentellen Design zu berücksichtigen und gleichzeitig die Anzahl der Attribute möglichst gering zu halten, da die Menge der Entscheidungsalternativen exponentiell mit der Zahl der Attribute und ihren Ausprägungen steigt und somit zu einem deutlich erhöhten Befragungsaufwand führt (BACKHAUS et al., 2011). Diese Voraussetzung wiegt in diesem Kontext umso schwerer, als dass das Arbeitgeberimage grundsätzlich durch eine Vielzahl potenziell entscheidungsrelevanter Arbeitsplatzmerkmale gekennzeichnet werden kann (vgl. Abschnitt 2.5.1). Das experimentelle Verfahren hat sich jedoch gerade an dieser Stelle als vorteilhaft erwiesen, da von dieser Vielzahl abstrahiert werden konnte, indem den Befragten mitgeteilt wurde, dass die zur Wahl vorliegenden Stellenangebote bis auf die Experimentalvariablen identisch sind (vgl. GRUND, 2003). Aus methodischer Perspektive lässt sich die begrenzte Anzahl der berücksichtigten Arbeitsplatzmerkmale auch deshalb rechtfertigen, da gezeigt werden konnte, dass Befragte ein konsistentes Antwortverhalten trotz hoher Komplexität erreichen, indem sie wenige zentrale Merkmale zu ihrer Entscheidungsfindung heranziehen (AUSPURG et al., 2009).

Neben der Anzahl wurden auch weitere inhaltlich-methodische Kriterien bei der Auswahl von Arbeitsplatzmerkmalen berücksichtigt. Demzufolge müssen die Eigenschaften relevant, beeinflussbar und unabhängig sein. Hinzu kommt, dass die entsprechenden Ausprägungen realisierbar und kompensatorisch zueinander sein müssen (BACKHAUS et al., 2011). Auf diese Weise wurden insgesamt fünf Arbeitsplatzmerkmale ausgewählt, wobei vier Eigenschaften jeweils zwei und eine Eigenschaft drei Ausprägungen haben. Bei diesen Arbeitsplatzmerkmalen handelt es sich durchweg um qualitative Attribute mit nominaler Skalierung.

Im Anschluss an die Definition des Untersuchungsobjektes wurde das experimentelle Design festgelegt. Generelles Ziel ist es, ein effizientes Design zu entwickeln, wobei dieses umso effizienter ist, je geringer die Varianz und die Kovarianz der geschätzten Nutzenparameter sind (HUBER und ZWERINA, 1996). Die Autoren führen neben der Orthogonalität der Eigenschaftsausprägungen die Ausgewogenheit der Ausprägungen und der Nutzenwerte sowie die minimale Überschneidung als Kriterien für effiziente Designs auf. Die hier gewählte Vorgehensweise orientierte sich eng am sog. Mix-and-Match-Verfahren von CHRZAN und ORME (2000). Demzufolge wurden auf der Basis der relevanten Arbeitsplatzmerkmale und ihrer Ausprägungen zunächst die Alternativen, sog. Stimuli, gebildet. Dies geschah, indem die entsprechenden Ausprägungen systematisch miteinander kombiniert wurden. Diese Kombination kann grundsätzlich auf zwei Arten erfolgen. Wenn jede Ausprägung eines Attributs mit jeder anderen Ausprägung der übrigen Attribute verknüpft wird, so liegt ein vollständiges Design vor. Allerdings führen vollständige Designs bereits bei einer geringen Zahl von Attributen und Ausprägungen zu einer sehr hohen Menge an Alternativen. Um eine zu hohe Anzahl zu vermeiden, werden häufig fraktionierte orthogonale Designs verwendet, bei denen unterstellt wird, dass Wechsel- 
wirkungen zwischen den einzelnen Attributen nicht auftreten. Es hat sich jedoch gezeigt, dass fraktionierte Designs selbst dann zu validen Ergebnissen führen, wenn Interaktionen relevant sind, sodass sie insgesamt als sehr robust einzustufen sind (VÖLCKNER et al., 2008). Hinzu kommt, dass eine ausreichende Stichprobengröße den grundsätzlich bestehenden Verlust an statistischer Information aufwiegen kann, wodurch auch bei reduzierten Designs annähernd gleiche statistische Eigenschaften wie bei vollständigen Designs erreicht werden (LOUVIERE et al., 2000). Vor diesem Hintergrund wurde auch in dieser Arbeit ein fraktioniertes orthogonales Untersuchungsdesign verwendet, dessen Erstellung auf dem Konstruktionsplan von KUHFELD (o.J.) beruhte. Hieraus ergaben sich insgesamt 12 Profile und genauso viele Wahlaufgaben, sog. Choice-Sets. Die Anzahl der Stimuli pro Choice-Set wurde dabei auf drei festgelegt, da die Auswahlentscheidung einen vergleichsweise hohen Komplexitätsgrad aufweist und die Befragten nicht durch zu viele Alternativen in einem Choice-Set überfordert werden sollten (VÖLCKNER et al., 2008). Zudem entspricht eine geringe Anzahl gleichzeitig zu bewertender Arbeitgeberimages eher dem Entscheidungskontext bei der Arbeitgeberwahl.

Neben der Anzahl pro Choice-Set wurde festgelegt, aus welchen Alternativen ein Choice-Set bestehen soll (JOHNSON et al., 2006). Gemäß dem Mix-and-Match-Prinzip von CHRZAN und ORME (2000) wurden die Choice-Sets zunächst nach dem sog. Shifting-Prinzip erzeugt. Hierbei dienten die 12 Stimuli als Ausgangsbasis für jedes zu definierende Choice-Set. Die beiden anderen Alternativen werden hierbei dadurch realisiert, dass die Ausprägungen jedes Arbeitsplatzmerkmals jeweils um eine Stufe (von 0 auf 1, von 1 auf 2, von 2 auf 0 ) erhöht wurden. Danach wurden die Stimuli innerhalb der drei Profile unabhängig voneinander durchmischt und zufällig den 12 Choice-Sets zugewiesen. Anschließend wurden aus den zwölf Choice-Sets insgesamt vier Decks mit jeweils drei Wahlaufgaben gebildet, die ebenfalls randomisiert auf die Befragten verteilt wurden. Die Beschränkung auf jeweils drei Auswahlentscheidungen pro Befragten wurde zum einen damit begründet, dass der zugrunde liegende Entscheidungskontext eine vergleichsweise hohe Komplexität aufwies, wodurch wiederum ein höheres Risiko bestand, die Befragten zu überfordern bzw. Ermüdungseffekte und Rückgriffe auf Entscheidungsheuristiken hervorzurufen (AUSPURG und LIEBE, 2011). Darüber hinaus gilt zwar generell, dass die Informationsmenge mit der Anzahl der abgefragten Wahlentscheidungen steigt (BACKHAUS et al., 2011). Gleichzeitig erweisen sich aber zusätzliche Wahlentscheidungen im Falle eines konsistenten Entscheidungsverhaltens der Befragten, erkennbar an der geringen Datenstreuung, als redundant und der Informationszuwachs nimmt ab (BROCKE, 2006).

Das Auftreten von Ausstrahlungs- oder Reihenfolgeeffekten innerhalb der vier Decks wurde mittels einer teilweisen Permutation kontrolliert, bei der sowohl absolute als auch relative Positionseffekte der Arbeitsplatzmerkmale ausbalanciert wurden, d.h. jedes Arbeitsplatzmerkmal bis auf das Gütesiegel war an erster, zweiter, dritter und vierter Stelle (= absolute Position) sowie vor und nach jedem anderen Arbeitsplatzmerkmal (= relative Position) platziert (BORTZ 
und DöRING, 2009). Da Gütesiegel in der Praxis, beispielsweise in Stellenanzeigen, zumeist unten dargestellt werden, wurden sie entsprechend immer an letzter Stelle aufgeführt.

Im nächsten Schritt war festzulegen, ob jedes Choice-Sets eine sog. Nicht-Wahl-Option, auch als None-Option bezeichnet, enthalten sollte. Bei dieser Option haben die Befragten die Möglichkeit, keine der in einem Choice-Set angebotenen Alternativen zu wählen. Aus methodischer Sicht erweist sie sich deshalb als vorteilhaft, da sie zur Realitätsnähe der Wahlaufgaben beiträgt (VÖLCKNER et al., 2008). So ist es im Hinblick auf die Arbeitgeberwahl plausibel, dass potenzielle Bewerber bestimmte Arbeitgeberimages als inakzeptabel beurteilen und sich folglich gegen die entsprechenden Arbeitgeber entscheiden. Gleichwohl birgt diese Option auch einen gewissen Nachteil, da aus den mit der None-Option bewerteten Choice-Sets keine statistischen Informationen über den Einfluss von Arbeitsplatzmerkmalen auf die Wahlentscheidung gewonnen werden können, wodurch die Nutzenschätzung instabiler wird (BACKHAUS et al., 2011). Ebenso könnte die None-Option eher von Befragten gewählt werden, die eine schwierige Entscheidungssituation umgehen wollen, woraus sich wiederum negative Konsequenzen für die Validität ergeben (HIMME, 2009). Die aufgezeigte Problematik wurde hierbei mittels einer separat abgefragten None-Option gelöst, indem die Befragten zunächst gebeten wurden, die am meisten präferierte Alternative zu wählen, und anschließend gefragt wurden, ob sie sich auch dann für die gewählte Alternative entscheiden würden, wenn sie sich für keine der im Choice-Set aufgeführten Alternativen entscheiden könnten. Durch das anfängliche „forcedchoice"-Format wurde zum einen verhindert, dass die Befragten die None-Option als bequeme Ausweichmöglichkeit nutzen. Und zum anderen wurde hierdurch sichergestellt, dass die als inakzeptabel befundenen Alternativen von den Befragten auch als solche gekennzeichnet wurden, um validitätsmindernde Effekte von vornherein auszuschließen.

\subsection{Methodenwahl für die Messung des dritten Teilmodells}

Basierend auf den im dritten Teilmodell aufgestellten Forschungshypothesen wurde ein Kausalmodell entwickelt, um den Einfluss der einzelnen Determinanten auf die Bewerbungsintention zu analysieren. Die darin postulierten Wirkbeziehungen bezogen sich zugleich auf hypothetische Konstrukte und wurden somit anhand latenter Variablen abgebildet. Solche latenten Variablen sind zugleich dadurch gekennzeichnet, dass es sich dabei um abstrakte, nicht direkt messbare bzw. beobachtbare Konstrukte handelt (WEIBER und MÜHLHAUS, 2010). Ihre Operationalisierung erfolgte daher mittels einzelner oder mehrerer direkt beobachtbarer Indikatoren, von denen zugleich angenommen wurde, dass sie mit dem zugrunde liegenden Konstrukt kausal eng zusammenhängen und dieses adäquat abbilden. Aus methodischer Sicht sind Strukturgleichungsmodelle an dieser Stelle deshalb besonders geeignet, da sie die Abhängigkeitsstrukturen zwischen latenten Variablen (im Strukturmodell) und die Messung theoretischer Konstrukte (im Messmodell) in einem einzigen Verfahren integrieren (HAIR et al, 2012). 
Zur Schätzung von Strukturgleichungsmodellen liegen grundsätzlich zwei unterschiedliche statistische Analysemethoden vor: die Kovarianzstrukturanalyse und die Partial-Least-Square-Analyse (PLS). Ein ausführlicher Methodenvergleich zwischen den beiden Ansätzen ist beispielsweise bei WeIBER und MÜHLhaus (2010) zu finden. Demzufolge liegt der Kovarianzstrukturanalyse eine ganzheitliche und simultane Schätzung der gesamten Kausalstruktur eines Modells zugrunde, wofür die Reproduktion der Varianz-Kovarianz-Matrix anhand aller empirisch gemessenen Beziehungen zwischen den Messvariablen ausschlaggebend ist. Dabei greift das Verfahren auf die Maximum-Likelihood- Schätzung aller Parameter zurück, wofür wiederum eine multivariate Normalverteilung der Daten vorausgesetzt wird. Aus der Anwenderperspektive betrachtet, handelt es sich hierbei um ein konfirmatorisches Verfahren, bei dem der deduktive Schritt - Schluss vom Modell auf die zu erwartende Kovarianz der Indikatorvariablen - im Vordergrund steht und das somit primär zur Theorieevaluation geeignet ist (FASSOTT, 2005).

Demgegenüber werden innerhalb des PLS-Ansatzes die Ausgangsdaten möglichst genau geschätzt, wodurch der induktive Schritt von den Daten auf das Modell betont wird. Hieraus geht zugleich hervor, dass sich der PLS-Ansatz vor allem für Prognosezwecke eignet (HAIR et al., 2011). Die aufgezeigten algorithmischen Unterschiede münden zugleich in einer Reihe abweichender Anwendungsbedingungen, von denen speziell drei die hier getroffene Wahl der PLS-Schätzmethode begründen. So ist das zu überprüfende Modell zum einen als komplex anzusehen, da es sich aus vergleichsweise vielen Konstrukten zusammensetzt. Zum anderen wurden die hier verwendeten Messansätze teils weiter- und teils neuentwickelt, während etablierte Skalen eher eine Ausnahme bildeten. Darüber hinaus setzt der PLS-Ansatz keine Verteilungsannahmen für die Parameterschätzung voraus, was seine Anwendbarkeit insbesondere bei explorativen Studien, in denen die Normalverteilungsannahme häufig nicht gewährleistet ist, begünstigt (NITZL, 2010). Aus diesem Grund wird PLS als Soft Modelling bezeichnet (HENSELER und SARSTEDT, 2012).

\begin{tabular}{|c|c|c|}
\hline Kriterium & Varianzbasiert (PLS) & Kovarianzbasiert (LISREL) \\
\hline Analysetyp & Least-Square-Analyse & Kovarianzstrukturanalyse \\
\hline $\begin{array}{l}\text { Schätzprinzip und Verteilungs- } \\
\text { annahme }\end{array}$ & $\begin{array}{l}\text { iterative und nicht-iterative } \\
\text { KQSchätzung } \\
\text { (keine Verteilungsannahme = } \\
\text { „Soft Modelling“): Minimierung } \\
\text { der Residualvarianzen im Mess- } \\
\text { und Strukturmodell }\end{array}$ & $\begin{array}{l}\text { Maximum-Likelihood-Schätzung } \\
\text { (i.d.R. multivariate Normalvertei- } \\
\text { lungsannahme der Daten = „Hard } \\
\text { Modelling“): Minimierung des } \\
\text { Abstands zwischen modelltheo- } \\
\text { retischer und empirischer Kovari- } \\
\text { anzmatrix }\end{array}$ \\
\hline
\end{tabular}




\begin{tabular}{|c|c|c|}
\hline Kriterium & Varianzbasiert (PLS) & Kovarianzbasiert (LISREL) \\
\hline $\begin{array}{l}\text { Eigenschaften } \\
\text { der Schätzparameter }\end{array}$ & $\begin{array}{l}\text { konsistent, wenn Fallzahl und } \\
\text { Indikatorenanzahl hoch }\end{array}$ & konsistent \\
\hline Größe der Stichprobe & $\begin{array}{l}\text { mindestens das Fünf- bis Zehn- } \\
\text { fache an Fällen der Prädiktoren- } \\
\text { zahl der größten Regressions- } \\
\text { gleichung; kleine Stichproben oft } \\
\text { ausreichend }\end{array}$ & $\begin{array}{l}\text { mindestens das Fünf- bis Zehn- } \\
\text { fache an Fällen der gesamten Vari- } \\
\text { ablenzahl; minimale Empfehlung } \\
\text { von } 200 \text { bis } 800\end{array}$ \\
\hline Beziehungen im Messmodell & $\begin{array}{l}\text { Standard: reflektive und formative } \\
\text { Zusammenhänge }\end{array}$ & $\begin{array}{l}\text { Standard: reflektive Zusammen- } \\
\text { hänge }\end{array}$ \\
\hline Skalenniveau & keine Einschränkung & mindestens Intervallskalen \\
\hline Anwendung & explorativer Charakter & konfirmatorischer Charakter \\
\hline Modellbeurteilung & $\begin{array}{l}\text { nur partielle Gütekriterien } \\
\text { (Heuristik) }\end{array}$ & $\begin{array}{l}\text { sowohl partielle als auch globale } \\
\text { Gütekriterien (statistische Fit- } \\
\text { Maße) einsetzbar }\end{array}$ \\
\hline Verwendete Software & PLSGraph, SmartPLS, LVPLS & LISREL, AMOS, EQS, M-PLUS \\
\hline
\end{tabular}

Tab. 5-2: Methodenvergleich zwischen PLS und Kovarianzstrukturanalyse

(Eigene Darstellung, 2012, in Anlehnung an FASSOTT, 2005; BOSSOW-THIES und PANTEN, 2009; HAIR et al., 2011; WEIBER und MÜHLHAUS, 2011)

Im Vorfeld der PLS-Analyse wurden analog zur DCA vorbereitende Maßnahmen getroffen. So wurden für die hypothetischen Konstrukte zunächst die dazugehörigen Items generiert und anschließend deren Messmodelle spezifiziert. Hiernach wurde ein Pretest durchgeführt, um die Gütekriterien der verwendeten Skalen im Vorfeld der Hauptuntersuchung zu überprüfen. Erst nachdem valide und reliable Items und Skalen vorlagen, wurde das Mess- und Strukturmodell simultan mittels PLS geschätzt.

Bei der anfänglichen Spezifikation des Messmodells wird grundsätzlich zwischen reflektiven und formativen Messungen unterschieden (GÖTZ und LIEHR-GOBBERS, 2004). So wird das latente Konstrukt innerhalb des reflektiven Messmodells kausal als Ursache seiner beobachtbaren Indikatoren modelliert, wohingegen dieses bei einem formativen Messmodell als eine gewichtete Zusammensetzung bzw. Ursache seiner Indikatoren betrachtet wird. Während also bei einem reflektiven Messmodell eine Veränderung des latenten Konstruktes eine Veränderung der beobachtbaren Indikatoren bewirkt, ist das formative Messmodell dadurch gekennzeichnet, dass eine Veränderung von einem oder mehreren Indikatoren zu einer Veränderung des Konstruktes führt. Aus diesen gegensätzlichen Kausalitätsannahmen ergeben sich weiterführende psychometrische Implikationen (CHRISTOPHERSEN und GRAPE, 2009). So müssen die Indikatoren in einem reflektiven Messmodell hoch miteinander korrelieren, wenn sie vom gleichen Konstrukt reflektiert werden, und zudem austauschbar sein, da sie allesamt durch ein 
und dasselbe Konstrukt verursacht werden. Auf diesen Interkorrelationen basiert zugleich das Gütekriterium Cronbachs Alpha als Maß für die interne Konsistenz einer Skala, wobei hier vorausgesetzt wird, dass die beobachtbaren Indikatoren auch tatsächlich unterschiedliche Folgen des zugrunde liegenden Konstruktes erfassen und nicht lediglich inhaltliche Umformulierungen eines einzigen Indikators darstellen (WEIBER und MüHLHAUS, 2010). Zudem sind reflektive Messmodelle dadurch gekennzeichnet, dass jeder beobachtbare Indikator mit einem systematischen und einem zufälligen Messfehler behaftet ist, was wiederum bedeutet, dass ein einzelner Indikator das Konstrukt nicht vollständig erfassen kann (GÖTZ und LIEHR-GOBBERS, 2004). Demgegenüber sind bei formativen Messmodellen hohe Interkorrelationen zwischen den Indikatoren zwar grundsätzlich möglich, jedoch werden sie nicht als notwendig vorausgesetzt (CHRISTOPHERSEN und GRAPE, 2009). Die Veränderung eines beobachtbaren Indikators kann hier indes durch die gleichzeitige Veränderung eines anderen Indikators ersetzt werden und damit in einer unveränderten Konstruktausprägung resultieren. Zudem kann die Skalenkonstruktion nicht nach den Kriterien der klassischen Testtheorie erfolgen, da die Eliminierung eines Indikators aus statistischen Gründen immer mit dem Verlust einer inhaltlichen Facette des zugrunde liegenden latenten Konstruktes einhergeht (WEIBER und MÜHLHAUS, 2010). Eine weitere Eigenschaft formativer Messmodelle ist darin zu sehen, dass der Messfehler dem latenten Konstrukt und nicht wie bei reflektiven Messmodellen dem beobachtbaren Indikator zugeordnet ist (GÖTZ und LIEHR-GOBBERS, 2004). Hieraus folgt, dass die aus den beobachtbaren Indikatoren resultierende Konstruktausprägung nicht dem wahren Konstruktwert entspricht, da die Indikatoren das latente Konstrukt nie vollständig erfassen können (BosSOW-THIES und PANTEN, 2009). Vor diesem Hintergrund sind weder die interne Konsistenz als Gütekriterium noch die Faktorenanalyse als statistische Analysemethode bei formativen Messmodellen zulässig. Die Güte eines formativen Messmodells lässt sich vielmehr danach beurteilen, ob alle relevanten Indikatoren als konstituierende Konstruktfacetten berücksichtigt worden sind (FASOTT und EGGERT, 2005).

Die Wahl einer korrekten Messmodellannahme ist deshalb so wichtig, da fehlspezifizierte Messmodelle zu verzerrten Ergebnissen von Strukturgleichungsmodellen führen können, was sich in den abweichenden Parameterschätzungen der postulierten Beziehungen auf der Konstruktebene zeigt (JARVIS et al., 2003). Dies kann wiederum in falschen Interpretationen und Schlussfolgerungen hinsichtlich der angenommen Kausalbeziehungen münden. Zudem führt die fälschliche reflektive Spezifizierung eines formativen Konstruktes dazu, dass inhaltlich relevante Indikatoren aufgrund der niedrigen Interkorrelationen eliminiert werden, wodurch sowohl die inhaltliche Validität des zugrunde liegenden latenten Konstruktes als auch seine Kriteriumsvalidität deutlich eingeschränkt werden (ALBERS und HILDEBRANDT, 2006).

Als typisches Beispiel formativer Messmodelle lassen sich vor allem die in der betriebswirtschaftlichen Forschung verwendeten Konstrukte, wie beispielsweise die Servicequalität, anführen. So wird die subjektiv wahrgenommene Servicequalität eines bestimmten Anbieters als 
gewichtete Summe mehrerer spezifischer Leistungen erfasst. Infolgedessen ist es mittels formativer Konstrukte zulässig, den Einfluss einzelner Servicebestandteile zu analysieren. Psychologische Konstrukte, wie z.B. Einstellung oder Leistungsmotivation, fungieren indes als typische Beispiele reflektiver Messmodelle (CHRISTOPHERSEN und GRAPE, 2009). Die in der Studie verwendeten Konstrukte wurden allesamt als reflektiv spezifiziert, sodass auf formative Messvorschriften im Folgenden nicht näher eingegangen wird.

Im Anschluss an die Modellspezifikation wurden die Skalierungsverfahren für die modellrelevanten Konstrukte festgelegt. Obwohl sich die verwendeten Skalen größtenteils eng an den bereits bestehenden Messansätzen orientierten, mussten sie an die zugrunde liegende Fragestellung angepasst werden. Diese Modifikationen betrafen zum einen die inhaltliche Zusammensetzung der Items zu einer Skala und zum anderen die Zuordnung der Skalenwerte. So wurde bei der Zusammenstellung der Items darauf geachtet, sowohl positive als auch negative Formulierungen aufzunehmen, um bestimmte Antworttendenzen, wie beispielsweise die sog. Ja-Sage-Tendenz, zu vermeiden (BORG und STAUFENBIEL, 2007). Darüber hinaus wurden fast durchgängig 5-stufige Ratingskalen verwendet, um einerseits eine ausreichende Differenzierungsfähigkeit der Skalen sicherzustellen und andererseits die Befragten nicht mit unterschiedlichen bzw. zu kleinteilig gewählten Antwortformaten zu überfordern (BORTZ und DöRING, 2009). Aufgrund der ungeraden Zahl an Antwortkategorien wurde die Mittelkategorie als neutrale Position integriert, die es den Befragten ermöglichte, sich tatsächlich neutral bzw. ambivalent hinsichtlich eines Merkmals oder Sachverhaltes zu positionieren. Eine solche Kategorie ist aus methodischer Sicht allerdings nicht unproblematisch, da sie auch dann gewählt werden könnte, wenn die Befragten beispielsweise aus Unkenntnis eine indifferente Meinung haben (WotTAWA, 2003). Die sog. Ambivalenz-Indifferenz-Problematik hätte zwar mittels einer expliziten Ausweichkategorie (z.B. „weiß nicht“") umgangen werden können, allerdings wäre auch diese Option mit einem Nachteil verbunden. So wird die „Weiß-nicht“-Kategorie von den Befragten nicht nur dann gewählt, wenn sie zu den jeweiligen Items tatsächlich keine Meinung oder Einstellung haben, sondern beispielsweise auch aus Gründen der Bequemlichkeit. Hinzu kommt, dass eine „Weiß-nicht“-Kategorie bei den Befragten den Eindruck suggerieren kann, nur dann von dieser Antwortkategorie abweichen zu dürfen, wenn sie ganz sicher sind oder die „richtige“ Antwort kennen (BORG und STAUfENBIEL, 2007). Da es sich bei den in der Studie verwendeten Skalen nicht um Faktenwissen, sondern um die subjektive Einschätzung von den als grundsätzlich vertraut angenommenen Merkmalen und Sachverhalten handelte, wurde aus den oben genannten Gründen auf diese Antwortkategorie verzichtet.

Ferner ist zu beachten, dass die Verwendung von Ratingskalen grundsätzlich mit einer messtheoretischen Problematik verknüpft ist, da ihr Skalenniveau bislang nicht abschließend festgelegt wurde (BORG und STAUFENBIEL, 2007). Hierbei geht es um die als problematisch eingeschätzte Annahme, inwieweit die Kategorien einer Ratingskala als äquidistant, also als intervallskaliert anzusehen und somit auch für statistische Analysen mittels parametrischer Verfahren geeignet 
sind. In dieser Untersuchung wird die Position von BORTZ und DÖRING (2009) vertreten, der zufolge implizite messtheoretische Annahmen aufrechterhalten werden können, solange die mittels Ratingskalen erzielten statistischen Ergebnisse inhaltlich sinnvoll zu interpretieren sind und sich bereits in der Forschungspraxis bewährt haben.

\subsection{Operationalisierung der Modellkonstrukte}

Unter dem Begriff der Operationalisierung wird grundsätzlich „die Summe der Anweisungen (Operationen), mit deren Hilfe ein hypothetisches Konstrukt (theoretischer Begriff) über beobachtbare Sachverhalte (Indikatoren) erfasst und gemessen werden soll (Messvorschrift)“" verstanden (WEIBER und MÜHLHAUS, 2010: 86). Demzufolge wird nachfolgend für jedes Modellkonstrukt einzeln erläutert, anhand welcher Items bzw. Skalen es operationalisiert wurde und welche Gütekriterien diesen zugrunde lagen.

\subsubsection{Operationalisierung des Arbeitgeberimages}

Das Arbeitgeberimage wird hier in Anlehnung an TEUFER (1999) als die Wahrnehmung eines Unternehmens bezogen auf die Ausprägungen seiner Arbeitsplatzmerkmale definiert. Die empirische Forschung zur Bedeutung verschiedener Arbeitsplatzmerkmale basiert zugleich auf einer Vielzahl von Studien, die in ihrer methodischen Umsetzung stark variieren und teils konträre Ergebnisse hervorbringen, sodass für die Auswahl und Operationalisierung der als entscheidungsrelevant angenommenen Arbeitsplatzmerkmale bislang keine einheitlichen Kriterien bzw. Instrumente vorliegen (vgl. Abschnitt 2.5.1). Vor diesem Hintergrund erfolgte die Bestimmung der relevanten Arbeitsplatzmerkmale und ihrer Ausprägungen mittels systematischer Literaturrecherchen und auf Basis von Expertengesprächen, breit angelegter Sichtung von Stellenausschreibungen und Arbeitgeberimagewerbung in einschlägigen Medien sowie empirischer Überprüfung im Pretest. Diesem Vorgehen lagen zugleich mehrere aufeinanderfolgende Teilschritte zugrunde. Zuerst wurden aus den empirischen Studien möglichst viele Arbeitsplatzmerkmale und Eigenschaftsausprägungen extrahiert, die im zweiten Schritt dahingehend geprüft wurden, ob sie den theoriegeleiteten Überlegungen einerseits und den oben dargestellten Kriterien an ein DC-Erhebungsdesign andererseits entsprechen. Bei den Eigenschaftsausprägungen der Arbeitsplatzmerkmale wurde insbesondere darauf geachtet, sehr realitätsnahe Formulierungen zu verwenden. Auf dieser Grundlage wurden folgende Arbeitsplatzmerkmale mit ihren jeweiligen Ausprägungen ausgewählt: Karriere- und Aufstiegschancen, Vereinbarkeit von Beruf und Familie bzw. Privatleben, Bezahlung, Arbeitsplatzsicherheit sowie Gütesiegel. Weitere potenziell bedeutsame Attribute, wie beispielsweise Weiterbildungsmöglichkeiten, wurden indes nicht erfasst, um die experimentelle Balance zwischen Komplexität und Vollständigkeit zu wahren. 


\subsubsection{Operationalisierung der Karriere- und Aufstiegschancen}

Die Karriere- und Aufstiegschancen wurden in Anlehnung an die bereits häufig verwendete Formulierung ,gute Aufstiegs- und Entwicklungsmöglichkeiten“ erfasst (vgl. z.B. GROBE, 2003; MALMENDIER, 2006; BÖTTGER, 2012). Die zweite Eigenschaftsausprägung wurde indes inhaltlich auf den langfristig gleichbleibenden Charakter der potenziell zu besetzenden Position ausgerichtet, wobei die entsprechende Formulierung möglichst positiv ausgestaltet wurde, um dem Kriterium der Realitätsnähe weitestgehend gerecht zu werden. Aus diesem Grund wurde auch davon abgesehen, auf die bereits erprobte Formulierung aus der conjointanalytischen Studie von WiLTINGER (1997) zurückzugreifen, die dort inhaltlich als „,kein Karriereplan, schlechte Aufstiegschancen“ umgesetzt wurde.

\subsubsection{Operationalisierung der Vereinbarkeit von Beruf und Familie}

Die Vereinbarkeit von Beruf und Familie bzw. Privatleben wurde mittels der in der Unternehmenspraxis weit verbreiteten Formulierung zur Arbeitszeitregelung erfasst. Dementsprechend stand „flexiblen Arbeitszeiten mit fester Wochenstundenzahl“ eine „Tätigkeit, die überdurchschnittliches Engagement und hohe Einsatzbereitschaft (z.B. Bereitschaft zur Wochenendarbeit bzw. Reisetätigkeit erfordert“" gegenüber.

\subsubsection{Operationalisierung der Bezahlung}

Im Gegensatz zu anderen empirischen Studien wurde das Arbeitsplatzmerkmal Bezahlung nicht über die Einkommenshöhe operationalisiert, sondern anhand der Gehaltsstruktur erfasst. Dies lag darin begründet, dass die absolute Gehaltshöhe in den unterschiedlichen Branchenzweigen der Ernährungsindustrie teilweise stark variiert und sich deshalb nur schwer anhand einiger weniger Ausprägungen abbilden lässt. Zudem finden potenzielle Bewerber in der frühen Phase des Personalmarketingprozesses in der Regel keine genauen Angaben zur tatsächlichen Einkommenshöhe. Infolgedessen wurde die Bezahlung hier anhand der zugrunde liegenden Gehaltsstruktur gemessen, wobei hier zwischen einem branchenüblichen, durchschnittlichen Festeinkommen und einer leistungsabhängigen Vergütung differenziert wurde (vgl. TUSCHKE, 2011). Erste Hinweise dafür, dass es sich der Entgeltstruktur um ein entscheidungsrelevantes Arbeitsplatzmerkmal handelt, liefert beispielsweise die Studie von KOYS et al. (1989), in der eine leistungsorientierte Bezahlung insbesondere von Mitarbeitern mit einer positiven Selbsteinschätzung ihrer eigenen Leistung präferiert wird. 


\subsubsection{Operationalisierung der Arbeitsplatzsicherheit}

Auch bei der Arbeitsplatzsicherheit wurde auf die häufig anzutreffende Formulierung zur Vertragsregelung zurückgegriffen. Zum Einsatz kamen die beiden Eigenschaftsausprägungen ,ein befristetes Arbeitsverhältnis“ versus „,ein unbefristetes Arbeitsverhältnis“. Eine solche Operationalisierung ist zugleich an die Untersuchung von SCHWOERER und ROSEN (1989) angelehnt, die allerdings zwischen einer willkürlichen und einer zu begründenden Kündbarkeit des Arbeitsverhältnisses unterscheiden.

\subsubsection{Operationalisierung der Gütesiegel}

Für die Ausprägungen dieses Arbeitsplatzmerkmals wurden aus den oben dargestellten forschungsökonomischen Gründen insgesamt zwei Gütesiegel - „audit berufundfamilie“ sowie „Top Job“ - ausgewählt, die aufgrund ihrer inhaltlichen Beschaffenheit und ihres Verbreitungsgrades am besten zu den mittelständischen Unternehmen der Ernährungsindustrie passen. Darüber hinaus wurde die Ausprägung „kein Gütesiegel“ als Referenzkategorie in das Teilmodell aufgenommen.

\begin{tabular}{|l|l|}
\hline Arbeitsplatzmerkmale & \multicolumn{1}{l|}{ Merkmalsausprägungen } \\
\hline Entwicklungs- und Aufstiegschancen & $\begin{array}{l}\text { - fest vorgegebene Position mit langfristig gleich- } \\
\text { bleibendem Aufgabenbereich } \\
\text { gute Aufstiegs- und Entwicklungsmöglichkeiten }\end{array}$ \\
\hline Vereinbarkeit von Beruf und Familie/Privatleben & $\begin{array}{l}\text { - Tätigkeit mit überdurchschnittlichem Engagement } \\
\text { und hoher Einsatzbereitschaft } \\
\text { flexible Arbeitszeiten mit fester Wochenstundenzahl }\end{array}$ \\
\hline Gehaltsstruktur & $\begin{array}{l}\text { - festes, branchenübliches Gehalt } \\
\bullet \text { leistungsorientierte Bezahlung }\end{array}$ \\
\hline Arbeitsplatzsicherheit & $\begin{array}{l}\text { - befristetes Arbeitsverhältnis } \\
\text { Gunbefristetes Arbeitsverhältnis }\end{array}$ \\
\hline
\end{tabular}

Tab. 5-3: Arbeitsplatzmerkmale und ihre Ausprägungen im Discrete-Choice-Modell

(Eigene Darstellung, 2012)

Nach der Bearbeitung der drei Wahlaufgaben sollten die Befragten auf einer dichotomen Skala zunächst angeben, ob sie die Entscheidungsfindung als schwierig empfunden haben. Ferner wurde erfragt, ob die beiden Gütesiegel bereits vor der Teilnahme an der Studie bekannt waren. Abschließend sollten die Befragten auf einer fünfstufigen Ratingskala ( 1 = völlig unwichtig bis 
$5=$ sehr wichtig) einschätzen, inwieweit sie die Auszeichnung ihres zukünftigen Arbeitgebers mit einem Gütesiegel generell als bedeutsam für sich erachten.

\subsubsection{Operationalisierung der Berufsorientierungen}

Die Berufsorientierungen, definiert als übergeordnete Motivationstendenzen, wurden mit je einem Item für Karriereorientierung, Freizeitorientierung und alternatives Engagement gemessen (Rosenstiel und Nerdinger, 2000). Dabei handelte es sich um Typenbeschreibungen, bei denen die Befragten auf einer siebenstufigen Skala angeben sollen, inwieweit sie den dort getroffenen Aussagen zustimmen $(1=$ stimme überhaupt nicht zu bis $7=$ stimme voll und ganz $\mathrm{zu})$. Grundsätzlich sind bei den Berufsorientierungen unterschiedliche Konstellationen denkbar. So können die zugrunde liegenden Motivationstendenzen zum einen miteinander konkurrieren, wenn beispielsweise zwei oder alle drei Berufsorientierungen etwa gleich ausgeprägt sind. Zudem kann eine Motivationstendenz eindeutig dominieren, wenn eine Berufsorientierung wesentlich stärker ist als die beiden anderen. Schließlich können sich primäre und sekundäre Motivationstendenzen herauskristallisieren, wenn eine klare individuelle Rangordnung der Berufsorientierungen erkennbar wird (BLICKLE, 1999). Eine methodisch nicht unumstrittene Besonderheit dieses Konstruktes besteht darin, dass jede Berufsorientierung anhand einer Single-Item-Skala erhoben wird. Eine solche Operationalisierung erscheint in diesem Falle jedoch deshalb als legitim, da in zahlreichen, auch längsschnittlichen Untersuchungen gezeigt werden konnte, dass die mit einem Item gemessenen Berufsorientierungen sich als valide Prädiktoren von unterschiedlichen berufsrelevanten Kriterien, wie beispielsweise Berufserfolg und berufliches Engagement, erwiesen haben (MAIER et al., 2009). Vor diesem Hintergrund wurden die Berufsorientierungen auch in dieser Studie mittels einer Single-Item-Skala gemessen.

\subsubsection{Operationalisierung der Konstrukte zur Vorhersage der Bewerbungsabsicht}

Die Formulierung der nachstehenden Konstrukte Arbeitgeberattraktivität, Prestige, Erfolgswahrscheinlichkeit der Bewerbung sowie Bewerbungsabsicht orientierte sich eng an den von AJZEN (2006) vorgelegten Konstruktionsrichtlinien für einen Fragebogen zur Theorie des geplanten Verhaltens. Gleichzeitig wurden diese formalen Kriterien auf die bereits vorhandenen und empirisch bewährten Konstrukte zur Arbeitgeberwahl übertragen, um die relevanten Modellkomponenten möglichst valide zu erfassen. Sie wurden fast ausschließlich mittels sog. Likert-Items erfasst, wobei die jeweilige Itemanzahl von eins bis vier variierte.

Die Entscheidung darüber, ob die reflektiven Konstrukte anhand einer Single-Item-Skala oder Multi-Item-Skala abzubilden waren, wurde von mehreren methodischen und praktischen Gesichtspunkten abhängig gemacht. Die Single-Item-Messung über ein sog. Globalitem kam dann 
zum Einsatz, wenn es sich um einen konkreten Bewertungsgegenstand handelte, der einen für alle Befragten einheitlichen Bedeutungsgehalt aufwies (FUCHS und DiAMANTOPOULOS, 2010). So erweisen sich Single-Item-Messungen deshalb als sinnvoll, da sie das Risiko von Ermüdungseffekten minimieren, was wiederum zu einer geringeren Abbruchquote bei Befragungen führt (BERGKVIST und ROSSITER, 2007). Darüber hinaus wird mithilfe von SingleItem-Messungen vermieden, mehrere inhaltlich redundante Items zu erzeugen. Bei den Befragten führt es andernfalls dazu, dass sie lediglich die zuerst genannten Items angemessen beantworten, während sie die noch folgenden Items entweder gänzlich auslassen oder aufgrund einer hohen Antwortkonsistenz identisch beantworten (WEIBER und MÜHLHAUS, 2010).

Die verwendeten Multi-Item-Skalen, die sich aus mindestens zwei und maximal vier Items zusammensetzten, basierten auf einer Likert-Skalierung, bei der die verschiedenen Antwortwerte additiv zu einem Skalengesamtwert verrechnet wurden (BORG und STAUFENBIEL, 2007). Beim Antwortformat wurde vorwiegend das charakteristische Zustimmungs-Ablehnungskontinuum verwendet, das auf einer fünfstufigen unipolaren Ratingskala mit den verbalen Etikettierungen 1 (= trifft gar nicht zu) bis 5 (= trifft vollständig zu) bzw. 1 (= stimme überhaupt nicht zu) bis 5 (= stimme voll und ganz zu) abgebildet war (BORTZ und DÖRING, 2009). Auf die davon abweichenden Antwortskalen wird in den entsprechenden Abschnitten explizit eingegangen. Eine Itemanalyse ergab zudem, dass zwei Items zu hohe Fremdtrennschärfen aufwiesen, d.h. mit anderen Skalenwerten höher korrelierten als mit ihrem eigenen. Durch Eliminierung dieser Items wurde deshalb versucht, verbesserte Skalen zu erstellen. Ferner wurde die Skala Branchenwissen aus formal-inhaltlichen Gründen um ein Item der Skala Branchenaffinität ergänzt. Tabelle 5-3 zeigt die deskriptiven Statistiken und Reliabilitäten aller im zweiten Teilmodell berücksichtigten Skalen, die durchweg zufriedenstellende Item- und Skalenwerte aufweisen.

\begin{tabular}{|l|r|r|r|r|}
\hline Konstrukte & \multicolumn{2}{c}{ M } & \multicolumn{2}{c|}{ SD } \\
\hline Arbeitgeberattraktivität & 3.40 & .93 & 4 & .93 \\
\hline Organisationales Prestige & 3.39 & .89 & 3 & .89 \\
\hline Erfolgswahrscheinlichkeit der Bewerbung & 3.51 & .74 & 2 & .89 \\
\hline Person-Organisation-Passung & 3.15 & 1.04 & 3 & .91 \\
\hline Bewerbungsabsicht & 3.13 & 1.19 & 1 & - \\
\hline $\begin{array}{l}\text { Wahrnehmung und Einschätzung von Alternativen } \\
\text { auf dem Arbeitsmarkt }\end{array}$ & 3.86 & .74 & 1 & - \\
\hline Branchenreputation & 2.96 & .65 & 3 & .74 \\
\hline Branchenwissen & 3.46 & .76 & 4 & .84 \\
\hline
\end{tabular}

Tab. 5-3: Deskriptive Statistiken und interne Konsistenzen (Cronbach's $\alpha$ ) aller im Teilmodell 3 berücksichtigten Skalen (N=671), Anmerkungen: k=Anzahl der Items (Eigene Darstellung, 2012). 


\subsubsection{Operationalisierung der Arbeitgeberattraktivität}

Gemäß ihrer Konstruktdefinition wurde die Arbeitgeberattraktivität in dieser Arbeit anhand von vier Items erfasst, in denen die Befragten angeben sollten, wie sie einen fiktiven Arbeitgeber bzw. eine Mitgliedschaft in einem fiktiven Unternehmen bewerten. Bei der Operationalisierung ging es primär darum, sowohl affektive als auch evaluative Bewertungen einzubeziehen. Ergänzend wurde mittels eines globalen Items gefragt, wie gut oder schlecht die Befragten die Anstellung bei einem bestimmten Unternehmen für sich persönlich einschätzen. Diese Itemformulierung ist deshalb so wesentlich, da sie AJZEN (2006) zufolge eine übergeordnete Verhaltensbewertung umfasst, in der affektive (z.B. angenehmen-unangenehmen) und kognitive (z.B. wichtig-unwichtig) Facetten gleichermaßen enthalten sind. Als Antwortformat wurde für alle vier Items ein fünfstufiges semantisches Differential verwendet. Bei der Skalenkonstruktion wurde explizit darauf geachtet, die Etikettierungen semantisch so eindeutig zu gestalten, dass sie von verschiedenen Befragten gleich verstanden werden und somit zu zuverlässigen Einstufungen führen (BORG und STAUFENBIEL, 2007). Das globale Item wurde mittels einer bipolaren Antwortskala erfasst, während den übrigen drei Items eine unipolare Antwortskala zugrunde lag. Die verwendeten Itemformulierungen sind an das empirisch bewährte Instrument von TURBAN und KEON (1993) angelehnt, wobei die Skala zur Steigerung der internen Konsistenz durch zwei selbst entwickelte Items ergänzt wurde.

\subsubsection{Operationalisierung des organisationalen Prestiges}

Auch die Operationalisierung des Konstruktes organisationales Prestige orientierte sich eng an den Vorgaben von AJZEN (2006). So wurde es anhand von drei Items gemessen, die die wahrgenommenen Verhaltenserwartungen der relevanten Bezugsperson erfassten. Inhaltlich basierten die Items auf der Studie von HighHOUSE et al. (2003) und thematisieren zum einen das Ansehen, das sich aus einer Mitgliedschaft im Unternehmen speist, und zum anderen die damit einhergehende Emotion Stolz.

\subsubsection{Operationalisierung der Erfolgswahrscheinlichkeit der Bewerbung}

Die wahrgenommene Verhaltenskontrolle lässt sich im Kontext der Arbeitgeberwahl als die subjektiv eingeschätzte Erfolgswahrscheinlichkeit der eigenen Bewerbung beim potenziellen Arbeitgeber auffassen. Hierbei wurde mittels eines Single-Items gemessen, inwieweit der Befragte die Erfolgsaussichten seiner Bewerbung bei einem bestimmten Unternehmen als positiv einschätzt. Zur Operationalisierung wurde auf das bereits erprobte Item aus den Studien von LEWANDOWSKI und LIEBIG (2004) und MALMENDIER (2006) zurückgegriffen. 


\subsubsection{Operationalisierung der Bewerbungsabsicht}

Für das Konstrukt der Bewerbungsabsicht liegen sehr unterschiedliche Messansätze vor (z.B. Aiman-Smith et al., 2001; CABle und Turban, 2003; HighHouse et al., 2003; Collins, 2007). In dieser Untersuchung wird die Bewerbungsabsicht in Anlehnung an den Intentionsbegriff von AJZEN (1991: 181) operationalisiert, demzufolge Intentionen als ,indicators of how hard people are willing to try, of how much of an effort they are planning to exert, in order to perform the behavior" definiert werden. Intentionen umfassen somit primär das Ausmaß an Anstrengung, das Personen in die Ausführung eines bestimmten Verhaltens zu investieren bereit sind (JONAS und DOLL, 1996). Vor diesem Hintergrund wurde bei diesem Konstrukt die Itemformulierung von AIMAN-SMITH et al. (2001) verwendet, die den per definitionem entscheidenden Aspekt einer Intention, nämlich den Grad der Anstrengung für eine bestimmte Handlung, angemessen widerspiegelt. Mit der gewählten Itemformulierung wird zudem eine wesentliche Voraussetzung der Korrespondenzannahme erfüllt, der zufolge die Intentionsmessung dem Spezifitätsniveau des zu prognostizierenden Verhaltens möglichst eng entsprechen soll (ECKES, 1996).

\subsubsection{Operationalisierung der Person-Organisation-Passung}

Auch zur Operationalisierung der supplementären Person-Organisation-Passung liegen grundsätzlich zwei unterschiedlicher Ansätze vor: die objektiven und subjektiven Messmethode (KRISTOF-BROWN et al., 2005). Bei der objektiven Variante werden absolute oder euklidische Distanzmaße herangezogen, die zwischen den separat erfassten Leistungsmerkmalen einer Organisation und den Werten bzw. Erwartungen einer Person bestehen (JUDGE und BRETZ, 1992). In der Literatur finden sich allerdings bislang kaum empirische Studien, in denen so vorgegangen wurde, sodass hierfür bislang keine verlässlichen Aussagen über die methodische Eignung und die entsprechenden Gütekriterien getroffen werden können. Demgegenüber wird beim subjektiven Verfahren direkt erfragt, ob und inwieweit die Erwartungen eines potenziellen Bewerbers mit dem Image einer Organisation übereinstimmen. Im Gegensatz zum objektiven Messansatz wurde die subjektive Methodik in einer Vielzahl empirischer Studien überprüft und entsprechend validiert (siehe für einen Überblick: ARTHUR et al., 2006). Infolgedessen wurde auch hier die subjektive Herangehensweise als geeignet angesehen. Bei der inhaltlichen Ausgestaltung wurden die Items von LIS (2010) verwendet, die ihrerseits aus der englischsprachigen Publikation Items von SAKS und ASHFORTH (2002) stammen und ins Deutsche übersetzt wurden. Die drei Items wurden inhaltlich leicht modifiziert, um die subjektiv wahrgenommene Kongruenz zwischen einem potenziellen Bewerber und einem Arbeitgeber noch besser herauszuarbeiten. 


\subsubsection{Operationalisierung der Alternativen auf dem Arbeitsmarkt}

Der Wahrnehmung und Einschätzung von Alternativen auf dem Arbeitsmarkt lag eine SingleItem-Messung zugrunde, die sich inhaltlich eng an die Itemformulierung von CABLE und JuDGE (1996) anlehnt. Der Einsatz einer Single-Item-Messung wurde auch hier damit begründet, dass die Gesamtheit des Konstruktes hierdurch ausreichend und eindeutig widergespiegelt wird (WEIBER und MÜHLHAUS, 2010).

\subsubsection{Operationalisierung der Branchenreputation}

Die Operationalisierung der Reputation basiert insgesamt auf sehr heterogenen Messansätzen. So wird es in den einschlägigen Studien als ein mehr- oder eindimensionales, formatives oder reflektives, bezugsgruppenspezifisches oder -übergreifendes Konstrukt erhoben (DAVIES et al., 2004). Bei einer näheren Betrachtung wird allerdings deutlich, dass es darin fast ausschließlich um einzelne Unternehmen als Reputationsträger geht (EISENEGGER und IMHOF, 2009). Demgegenüber existieren bislang nur vereinzelt Studien, in denen die Relevanz der nächsthöheren Ebene, nämlich der Branchenreputation untersucht wurde (ALBERSMEIER und SPILLER, 2009). EISENEGGER und KÜNSTLE (2003) zufolge lässt sich die Branchenreputation grundsätzlich auf unterschiedliche konstituierende Faktoren zurückführen: Neben der simultanen Einschätzung vieler Unternehmen einer bestimmten Branche kann die Bewertung entweder anhand einiger weniger Unternehmen bzw. eines Leitunternehmens oder lediglich anhand einiger Personen bzw. eines einzigen Leitmanagers erfolgen. Da die Ernährungsindustrie mittelständisch geprägt ist und der Bekanntheitsgrad einzelner Unternehmen dementsprechend eher gering sein dürfte, eignen sich die beiden zuletzt genannten Verfahren nicht dazu, die Branchenreputation adäquat zu erfassen. Ebenso besteht das Forschungsanliegen weniger darin, die einzelnen Reputationsbestandteile zu identifizieren, sondern vielmehr die Auswirkungen des Branchenrufs auf die Determinanten der Bewerbungsabsicht zu bestimmen. Infolgedessen wurde hier auf die Gesamtbewertung der zur Ernährungsindustrie gehörenden Unternehmen zurückgegriffen. Entsprechend wurden die drei Items eindimensional und global, d.h. auf die gesamte Branchen bezogen, angelegt. Dabei sollten die Befragten in Anlehnung an HAUTZINGER (2009) einschätzen, inwieweit der gesamten Ernährungsindustrie ein guten Ruf bzw. ein hohes Ansehen in der Öffentlichkeit zugeschrieben werden kann.

\subsubsection{Operationalisierung des Branchenwissens}

Auch für das Konstrukt des Branchenwissens existiert bislang kein etablierter einheitlicher Messansatz. In Anlehnung an TROMMSDORFF (2009: 79) wurde hier das subjektive Wissen über die Ernährungsindustrie als grundlegend erachtet, „das bei Bedarf zur Verfügung steht, 
sei es intern als gespeicherte Information, die durch Erinnern (Abrufen) verfügbar ist, sei es als externe Information, die durch Wahrnehmen (Aufnehmen) verfügbar wird“. Die Studien zum Arbeitgeberwahlverhalten stützen die Verwendung einer solchen Operationalisierung, obwohl sie bislang nur die Ebene eines einzelnen Unternehmens und nicht der gesamten Branche betrachteten (vgl. z.B. TURBAN et al., 1998; CABLE und TURBAN, 2003; COLLINS, 2007). Eine Ausnahme stellt die Untersuchung von HAUTZINGER (2009) dar, in der explizit das übergeordnete Branchenwissen gemessen wurde. In seiner Studie erwies sich die Skala mit einer internen Konsistenz von 90 als reliabel. Die drei Items aus seiner Studie wurden inhaltlich auf die Ernährungsindustrie angepasst und um ein weiteres Item ergänzt.

\subsubsection{Operationalisierung der Methoden zur externen Bewerberansprache}

Die externe Ansprache von potenziellen Bewerbern kann grundsätzlich über eine Vielzahl von Rekrutierungskanälen erfolgen (vgl. Abschnitt 2.3). Aus forschungsökonomischen Gründen konnten allerdings nicht sämtliche existierenden Informationsquellen in die Untersuchung aufgenommen werden, sodass eine an dem bereits erprobten Instrument von KANNING et al. (2009) angelehnte Auswahl getroffen wurde. Das Instrument wurde um einige weitere Items ergänzt, die aus aktuellen Studien zur Rekrutierung von Hochschulabsolventen stammten (WEITZEL et al., 2012). Die Bewertung der im Fragebogen berücksichtigten 13 Maßnahmen umfasste zugleich mehrere Kriterien: Neben ihrer grundsätzlichen Bekanntheit und der persönlichen Nutzungsintensität, jeweils dichotom mit ,ja“ oder nein“ erfasst, wurde ihre Nützlichkeit für die Arbeitgeberwahl auf einer fünfstufigen Ratingskala ( 1 = überhaupt nicht nützlich bis $5=$ sehr nützlich) abgefragt. Abschließend wurden die drei am meisten präferierten Maßnahmen mittels eines offenen Antwortformates erhoben.

\subsubsection{Operationalisierung des Involvements}

Zur Operationalisierung des persönlichen Involvements wurde auf das von LAURENT und KAPFERER (1985) entwickelte und in der marketingwissenschaftlichen Forschung häufig verwendete Instrument zurückgegriffen. Im Hinblick auf die vorangegangenen theoretischen Überlegungen wurde für das Produkt Stellenangebot angenommen, dass die Aspekte persönliche Relevanz, Identifikation bzw. persönliche Ausdrucksmöglichkeiten sowie Risikograd zu den wesentlichen inhaltlichen Bestandteilen zählen. Diese wurden hier in Anlehnung an SCHAEFER (2006) mit jeweils einem Item operationalisiert. Angesichts der zugrunde liegenden Konstruktdefinition von TROMMSDORFF (2009) einerseits und der Homogenität der verwendeten Items andererseits wurde das Konstrukt jedoch nicht wie bei LAURENT und KAPFERER (1985) mehrdimensional, sondern eindimensional angelegt und die Items somit als Indikatoren einer Multi-Item-Skala 
betrachtet. Zugleich wurde das persönliche Invovement entgegen der Annahme von SCHAEFER (2006) nicht formativ, sondern in Anlehnung an GÖTZ und LIEHR-GOBBERS (2004) als ein reflektives Konstrukt spezifiziert. Diese Spezifikation beruht darauf, dass es sich hierbei um ein psychologisches Konstrukt handelt, dessen Indikatoren inhaltlich denselben Kern besitzen, austauschbar sind und bei einer Elimination nicht die inhaltliche Aussage der zugrundeliegenden latenten Variablen verändern (CHRISTOPHERSEN und GRAPE, 2009).

Ergänzend zum persönlichen Involvement wurde das Situationsinvolvement herangezogen, das anhand der Nähe zur Entscheidungssituation, hier zum Studienabschluss, operationalisiert wurde (TROMMSDORFF, 2009). Als Indikatoren für das situationsspezifische Involvement dienten das aktuelle Fachsemester und die erwartete Semesteranzahl bis zum Studienende (MALMENDIER, 2006).

\subsubsection{Soziodemographische Variablen}

Zur angemessenen Beschreibung der Stichprobe wurden folgende demographische Merkmale erhoben: Alter, Geschlecht, Hochschul-, Abschluss- und Studienart, Fachrichtung, Studiengang, aktuelles Fachsemester sowie verbleibende Semesteranzahl bis zum Studienabschluss. Die Hochschulart wurde anhand der beiden Optionen $1=$ Fachhochschule und $2=$ Universität erfasst, für die Studienart wurden die Kategorien 1 = Bachelorstudium und 2 = Masterstudium verwendet. Für die Variable Studienart wurden als Antwortoptionen 1 = Vollzeitstudium, 2 = Fernstudium, 3 = berufsbegleitendes Studium sowie $4=$ duales Studium vorgegeben. Als Antwortkategorien für die Variable Fachrichtung dienten folgende alphabetisch geordnete Optionen: Agrarwissenschaften, Ingenieurwissenschaften, Naturwissenschaften, Sozialwissenschaften, Wirtschaftswissenschaften sowie Sonstige. Die Beschränkung auf die oben genannten Fachrichtungen beruhte darauf, dass ausschließlich Studierende dieser Fachdisziplinen als potenzielle Bewerber in der Ernährungsindustrie für die Untersuchung relevant waren. Des Weiteren wurde die vorhandene Berufserfahrung, z.B. in Form einer beruflichen Ausbildung oder studienrelevanter Praktika, sowie der aktuelle Status bei der Stellensuche erfasst. Bei der Variablen Berufserfahrung wurde zunächst dichotom ( 1 = ja, 2 = nein) erfragt, ob eine solche bei den Befragten grundsätzlich vorhanden ist. Anschließend wurde anhand der folgenden Antwortkategorien erfasst, wie sich die Berufserfahrung zeitlich zusammensetzt: 1 = weniger als 6 Monate, 2 =zwischen 6 Monaten und 1 Jahr, $3=$ mehr als 1 Jahr. Ergänzend wurde die Gesamtanzahl studienrelevanter Praktika erfragt. Die Variable aktuell auf Stellensuche wurde neben der dichotom ausgeprägten Antwortkategorie ( $1=$ ja, 2 = nein $)$ anhand folgender Antwortoptionen erfasst: 1 = Nebenjob, 2 = Praktikum, $3=$ Abschlussarbeit, $4=$ Festanstellung. 


\subsubsection{Kontrollvariablen}

Ergänzend zu den oben beschriebenen Experimentalvariablen wurden zwei Kontrollvariablen erhoben, die sich auf die Studienergebnisse hätten auswirken können: Affinität zur Ernährungsindustrie sowie Informationsstatus hinsichtlich der Arbeitgeberwahl. Wenngleich sich die Stichprobe ausschließlich aus Studierenden solcher Studiengänge zusammensetzte, in denen die Ernährungsindustrie ganz explizit ein potenzielles Berufsfeld darstellt, wurde zusätzlich die subjektiv empfundene Affinität der Befragten zur Ernährungsindustrie erhoben. Hierin wurde festgestellt, ob und inwieweit die fachlichen Interessen und Studienschwerpunkte der Befragten die grundsätzliche Aufnahme einer Tätigkeit in einem Unternehmen der Ernährungsindustrie ermöglichten. So könnte eine insgesamt geringe Bereitschaft für eine Tätigkeit in der Ernährungsindustrie, die ihrerseits primär durch ein abweichendes fachliches Qualifikationsprofil bedingt wurde, die postulierten Zusammenhänge beeinflussen. Zudem wurde der aktuelle Informationsstatus der Befragten im Hinblick auf ihre individuelle Beschäftigung mit dem künftigen Arbeitgeber erfasst. Dies lag darin begründet, dass es sich bei der Arbeitgeberwahl um eine extensive bzw. reflektierte Entscheidung handelt, die zugleich eine vorangehende Auseinandersetzung mit den eigenen Erwartungen bzw. Anforderungen voraussetzt (TROMMSDORFF, 2009). So erschien es beispielsweise plausibel, dass ein Befragter, der sich bislang nicht näher mit dieser Thematik beschäftigt hat, die für ihn relevanten Arbeitsplatzmerkmale nicht eindeutig benennen und infolgedessen die Ergebnisse durch ein willkürliches Ankreuzen der Alternativen im Auswahlset verfälschen könnte.

\subsection{Pretest}

Vor ihrem Einsatz in der Hauptstudie wurden die unten dargestellten Skalen in einer Voruntersuchung hinsichtlich der Kriterien Verständlichkeit und Eindeutigkeit der Items erprobt. Zu diesem Zwecke wurden insgesamt 84 Studierende der relevanten Studiengänge der Hochschule Osnabrück gebeten, den Pretest-Fragebogen auszufüllen und ggf. auf uneindeutige oder unverständliche Itemformulierungen hinzuweisen. Die Ergebnisse des Pretests wurden zudem dazu genutzt, die Reliabilität der Skalen mittels einer Itemanalyse zu überprüfen und diese anschließend zu optimieren. Hierbei wurde versucht, jedes Konstrukt durch mindestens drei Items zu operationalisieren, um eine ausreichende interne Konsistenz bei den entsprechenden Skalen sicherzustellen. Gleichzeitig wurde bei der Skalenoptimierung besonders darauf geachtet, sich wiederholende oder unverständliche Itemformulierungen zu entfernen, um den Bearbeitungsaufwand für die Befragten möglichst gering zu halten. Die nachfolgend dargestellten Messansätze basieren somit auf den im Pretest bereinigten Skalen. 


\subsection{Aufbau des Fragebogens}

Als Instrument der Datenerhebung wurde eine standardisierte schriftliche Befragung gewählt. Basierend auf der oben dargestellten methodischen Konzeption der DCA wurden insgesamt zwölf Fragebogenversionen konstruiert, die randomisiert auf die Befragten verteilt wurden. Grundsätzlich wurde bei der Fragebogenkonzeption darauf geachtet, einfache, eindeutige sowie neutrale und für den Untersuchungsgegenstand relevante Fragen- bzw. Itemformulierungen zu wählen (BORG und StAUfENBIEL, 2007). Der nach dem Pretest modifizierte Fragebogen umfasste insgesamt sechs inhaltlich zusammenhängende Fragenkomplexe, deren Bearbeitung insgesamt ca. 30 bis 40 Minuten beanspruchte. Eingeleitet wurde der Fragebogen mit einem Begleitschreiben, aus dem der Untersuchungszweck, die voraussichtliche Bearbeitungsdauer und Hinweise zur bei der Beantwortung der Fragen hervorgingen. Im ersten Abschnitt wurden die soziodemographischen Daten der Befragten erhoben. Hierauf folgte ein zweiter Abschnitt, in dem branchenspezifische Fragen sowie persönliches Involvement und Arbeitsmarktchancen thematisiert wurden. Im dritten Abschnitt wurden die Berufsorientierungen der befragten Personen erörtert. Im anschließenden Themenkomplex wurde das Discrete-Choice-Experiment (DCE) platziert, das sich für jeden Befragten aus einer einheitlichen Situationsbeschreibung, drei Choice-Sets mit jeweils drei Stellenangeboten zusammensetzte. Gemäß der voranstehenden Instruktion sollten sich die Befragten vorstellen, dass sie sich bereits am Ende ihres Studiums befinden und aktiv auf Stellensuche sind. Dabei lägen ihnen mehrere Stellenangebote von mittelständischen Unternehmen aus der Ernährungsindustrie vor, bei denen sämtliche fachlichen und regionalen Kriterien, wie beispielsweise Art der Tätigkeit oder Einsatzort, erfüllt wären. Unterschiede beständen indes hinsichtlich der Arbeitsplatzmerkmale Bezahlung, Arbeitsplatzsicherheit, Vereinbarkeit von Beruf und Familie bzw. Privatleben sowie Aufstiegs- und Entwicklungschancen. Zudem führten einige Unternehmen ein durch unabhängige Anbieter verliehenes Gütesiegel. Das Ziel eines solchen Vorgehens besteht darin, die experimentellen Variablen in den Fokus zu rücken und andere potenziell entscheidungsrelevante Arbeitsplatzmerkmale, wie beispielsweise den Standort des Unternehmens, als erfüllt zu definieren und somit konstant zu halten (vgl. z.B. BACKHAUS et al., 2002; BECKER et al., 2008; LIS, 2010). Innerhalb der fiktiven Unternehmensszenarien wurden die Experimentalvariablen tabellarisch aufgelistet, da diese Darbietungsform im Vergleich zu einer Fließtext-Formulierung übersichtlicher erschien und somit gerade bei komplexen Wahlaufgaben ein konsistentes Antwortverhalten begünstigen könnte (AUSPURG et al., 2009). Die Skalen zur Theorie des geplanten Verhaltens bildeten den fünften Fragenkomplex. Als Einstellungsobjekt diente hier wieder eine fiktive Stellenausschreibung eines mittelständischen Unternehmens aus der Ernährungsindustrie. Sie entsprach in ihrem inhaltlichen Aufbau gängigen Stellenausschreibungen mittelständischer Unternehmen und war zudem in ihrer optischen Gestaltung weitestgehend an die in der Praxis gängigen Formate angepasst. Ähnlich wie bei der DCA wurden auch hier die fachlichen und regionalen Kriterien als erfüllt vorgegeben. Abschließend wurden im sechsten Abschnitt Fragen zum Informations- 
verhalten der befragten Personen untergebracht. Innerhalb der jeweiligen Abschnitte wurden die Items in ihrer Anordnung so durchmischt, dass möglichst keine Kontexteffekte, wie beispielsweise Reihenfolge-Effekte, auftreten konnten.

1 Soziodemographische Variablen

2 Branchenspezifika, Involvement und Arbeitsmarktsituation

3 Berufsorientierungen

4 Discrete-Choice-Experiment

5 Determinanten der Bewerbungsintention

6 Informationsverhalten/Maßnahmen des Personalmarketings

Abb. 5-2: Fragebogenaufbau (Eigene Darstellung, 2012)

\subsection{Durchführung der Befragung und Stichprobenauswahl}

Die Befragung der Hauptstudie fand im Zeitraum von Oktober 2011 bis April 2012 statt. Da in dieser Arbeit das spezifische Arbeitgeberwahlverhalten potenzieller Bewerber in der Ernährungsindustrie im Fokus stand, beschränkte sich die Stichprobensauswahl auf diejenigen Merkmalsträger der Grundgesamtheit, deren Antworten als besonders relevant bzw. typisch hinsichtlich des Untersuchungsgegenstandes erachtet wurden (HAMMANN und ERICHSON, 2000). Diesem Forschungsziel entsprechend bestand die Grundgesamtheit aus Studierenden agrar-, ernährungs-, ingenieur- und wirtschaftswissenschaftlicher sowie technischer Studiengänge, die sich zum Befragungszeitpunkt in höheren Semestern befunden und somit unmittelbar vor einer Bewerbungsphase mit den damit einhergehenden Entscheidungsprozessen gestanden haben. Die Einschränkung der Grundgesamtheit auf akademisch ausgebildete Bewerber liegt zugleich darin begründet, dass diese Zielgruppe für die mittelständischen Unternehmen der Ernährungsindustrie von besonderem Interesse ist. Darüber hinaus verfügen solche Bewerber aufgrund der derzeit positiven Arbeitsmarktlage über einen größeren Entscheidungsrahmen, sodass die experimentell geschaffene Entscheidungssituation mit mehreren Alternativen durchaus realitätsnah erschien. Über einen fortgeschrittenen Studienverlauf sollte schließlich sichergestellt werden, dass die Beschäftigung mit der Arbeitgeberwahl für die Studierenden bereits zum Befragungszeitpunkt war oder in naher Zukunft auch tatsächlich relevant sein würde. Die hier getrof- 
fene Einschätzung, dass es sich bei diesen Studierendengruppen grundsätzlich um potenzielle Bewerber in der Ernährungsindustrie handelt, basiert zum einen auf Expertengesprächen mit Personalverantwortlichen und zum anderen auf aktuellen Stellenausschreibungen mittelständischer Unternehmen aus dieser Branche. Eine typische Auswahl hinsichtlich der Ernährungsindustrie wurde in den bisherigen empirischen Studien zur Arbeitgeberwahl nicht untersucht, sodass hierüber erstmalig branchenspezifische Erkenntnisse zu erwarten waren.

Als Erhebungsorte wurden insgesamt sechs Hochschulen, zwei Universitäten (Universität Kiel und Universität Göttingen) und vier Fachhochschulen (Hochschule Osnabrück, Fachhochschule Münster, Hochschule Bremerhaven und Hochschule Ostwestfalen-Lippe) festgelegt, die Studierende in den oben genannten Fachdisziplinen ausbilden. Die Auswahl von Fachhochschulen beruhte auf folgenden inhaltlichen Überlegungen. So wurden in den bisherigen deutschsprachigen Studien zur Arbeitgeberwahl überwiegend Universitätsstudierende untersucht, Studierende von Fachhochschulen spielten indes ungeachtet ihres hohen Stellenwertes auf dem Arbeitsmarkt bislang eine untergeordnete Rolle. Darüber hinaus wurde mit diesem Vorgehen versucht, die Stichprobe möglichst heterogen und somit varianzstark zu gestalten.

Die Fragebögen wurden in Lehrveranstaltungen nach einer kurzen Ansprache durch die Versuchsleiterin verteilt. Die Teilnahme an der Studie war freiwillig und wurde nicht entlohnt. Von den insgesamt 1013 verteilten Fragebögen, wurden 671 bearbeitet und zurückgegeben, woraus sich eine relativ hohe Rücklaufquote von 66 Prozent ergab. Die Stichprobenauswahl und die Erhebung gingen allerdings mit einigen problematischen Aspekten einher, die zu erwähnen sind. So ist die Befragung nicht als repräsentativ zu bezeichnen, da im Vergleich zu einer echten $\mathrm{Zu}-$ fallsstichprobe nicht jeder potenzielle, akademisch ausgebildete Bewerber in der Ernährungsindustrie die gleiche Wahrscheinlichkeit hatte, in die Stichprobe zu gelangen. Zudem könnte die regionale Fokussierung der Studienorte auf den nordwestdeutschen Raum bewirkt haben, dass die Stichprobe gegenüber anderen regionalen Standorten verzerrt ist. Eine repräsentative Abbildung der Grundgesamtheit in der Stichprobenauswahl wäre jedoch auch deshalb nicht möglich gewesen, da bisher keine veröffentlichten Angaben zur Grundgesamtheit der Bewerber in der Ernährungsindustrie existieren. Gleichwohl sind die aufgezeigten Einschränkungen hinsichtlich der Stichprobenrepräsentativität zu relativieren. So liegt der durchgeführten Untersuchung eine vergleichsweise große Anzahl von branchenspezifischen Befragungen zugrunde, woraus aussagekräftige Hinweise für die zentralen Forschungsfragen abgeleitet werden können. Darüber hinaus gibt es bislang keine Belege, aus denen sich bedeutsame regionale Unterschiede in den Bewerbererwartungen ableiten ließen. Ebenso konnten in inhaltlich vergleichbaren Studien keine Hinweise gefunden werden, aus denen eine systematische Verzerrung der Ergebnisse aufgrund fehlender Repräsentativität hervorging. Schließlich war es für die Modellüberprüfung wesentlich, möglichst typische und nicht im statistischen Sinne repräsentative Merkmalsträger bei der Stichprobenauswahl zu berücksichtigen. 


\subsection{Behandlung fehlender Werte}

Aufgrund der persönlichen und schriftlichen Bitte der Versuchsleiterin, den Fragebogen möglichst vollständig auszufüllen, waren lediglich einige wenige fehlende Werte, weniger als 5 Prozent, zustande gekommen. Obwohl es sich bei den fehlenden Werten um einen vollkommen zufälligen Datenausfall handelte, wurde bei der statistischen Analyse eine sehr restriktive Auswahl getroffen, in der ausschließlich vollständige Sätze in die Auswertung kamen, während Datensätze mit fehlenden Werten ignoriert wurden. Dieses Verfahren wurde gewählt, da es am ehesten dazu geeignet ist, unverzerrte Parameter zu ermitteln (GÖTHLICH, 2009). 
KAPITEL 6

\section{Ergebnisse der empirischen Untersuchung}

Im Folgenden werden zunächst die deskriptiven Ergebnisse der Gesamtuntersuchung vorgestellt. Daran schließen sich die Ergebnisse der Hypothesenprüfung an, die für jedes Teilmodell gesondert aufgeführt und erläutert werden. Das Kapitel schließt mit einer strukturierten Übersicht aller in dieser Arbeit erzielten Befunde.

\subsection{Beschreibung der Stichprobe}

Die im Folgenden dargestellten Ergebnisse basieren auf insgesamt 671 Befragungen. Knapp 60 Prozent der Befragten waren weiblich, ca. 40 Prozent männlich. Betrachtet man die Altersklassen, so finden sich unter den Befragten erwartungskonform vorwiegend Personen zwischen dem 21. und 26. Lebensjahr, wobei ihr Durchschnittsalter bei 24 Jahren lag. Ein Großteil studierte an einer Fachhochschule, ca. 20 Prozent der Befragten absolvierten ihr Studium an einer Universität. Analog dazu waren etwa drei Viertel der Befragten in einem Bachelorstudiengang eingeschrieben, während knapp ein Viertel einen Masterstudiengang absolvierte. Die Studierenden in den Bachelorstudiengängen befanden sich zum Befragungszeitpunkt durchschnittlich im letzten Drittel ihres Studiums: Sie haben durchschnittlich bereits 4,5 Fachsemester studiert und rechneten mit durchschnittlich 1,9 Fachsemestern bis zu ihrem Studienabschluss. Bei den Studierenden in den Masterstudiengängen ergab sich ein davon abweichendes Bild: Hier lag die durchschnittliche absolvierte Fachsemesteranzahl bei 1,6 Semestern, während die bis zum Studienabschluss verbleibende Fachsemesteranazahl im Durchschnitt 2,5 betrug. Die Studiengänge wurden drei Fachrichtungen zugeordnet: Agrar- und Ernährungswissenschaften (AEW), Lebensmitteltechnologie (LMT) und Betriebswirtschaftslehre (BWL). Knapp 88 Prozent der Befragten sind in etwa gleichen Anteilen den ersten beiden Fachrichtungen zuzuordnen, während Studierende der Betriebswirtschaft mit 12 Prozent eine mit Abstand kleinere Gruppe bildeten. 


\begin{tabular}{|c|c|c|c|}
\hline Merkmal & Ausprägung & $\begin{array}{l}\text { Absolute } \\
\text { Häufigkeiten }\end{array}$ & $\begin{array}{l}\text { Relative } \\
\text { Häufigkeiten (in \%) }\end{array}$ \\
\hline \multirow{2}{*}{ Geschlecht } & weiblich & 402 & 59,9 \\
\hline & männlich & 269 & 40,1 \\
\hline \multirow{5}{*}{ Altersklasse } & unter 21 Jahren & 47 & 7,0 \\
\hline & 21 bis 23 Jahre & 318 & 47,4 \\
\hline & 24 bis 26 Jahre & 217 & 32,3 \\
\hline & 27 bis 29 Jahre & 65 & 9,7 \\
\hline & 30 Jahre und älter & 24 & 3,6 \\
\hline \multirow{3}{*}{ Fachrichtung } & $\begin{array}{l}\text { Agrar-/ } \\
\text { Ernährungswissenschaften }\end{array}$ & 284 & 42,3 \\
\hline & Lebensmitteltechnologie & 308 & 45,9 \\
\hline & Betriebswirtschaft & 79 & 11,8 \\
\hline \multirow{2}{*}{ Hochschulart } & Fachhochschule & 547 & 81,5 \\
\hline & Universität & 124 & 18,5 \\
\hline \multirow{2}{*}{ Abschlussart } & Bachelor & 498 & 74,2 \\
\hline & Master & 173 & 25,8 \\
\hline Merkmal & $\begin{array}{l}\text { Mittelwert } \\
\text { gesamt }\end{array}$ & $\begin{array}{l}\text { Mittelwert } \\
\text { Bachelorstudium }\end{array}$ & $\begin{array}{l}\text { Mittelwert } \\
\text { Masterstudium }\end{array}$ \\
\hline Alter & 23,76 & 23,45 & 24,65 \\
\hline Fachsemester & & 4,45 & 1,62 \\
\hline $\begin{array}{l}\text { Fachsemester bis } \\
\text { Studienabschluss }\end{array}$ & & 1,88 & 2,46 \\
\hline
\end{tabular}

Tab. 6-1: Sozidemografische Merkmale der Stichprobe (Eigene Berechnungen, 2012) 


\subsection{Analyse des ersten Teilmodells: Informationsverhalten potenzieller Bewerber}

Das Forschungsziel des ersten Teilmodells besteht in einer explorativen Analyse des Informationsverhaltens potenzieller Bewerber in mittelständischen Unternehmen der Ernährungsindustrie. Somit wurde hierbei zum einen überprüft, wie der Bekanntheitsgrad, die individuelle Nutzungsintensität sowie die Nützlichkeit und Präferenz ausgewählter Maßnahmen des Personalmarketings durch die relevante Zielgruppe eingeschätzt wird. Zum anderen wurde der Forschungsfrage nachgegangen, inwieweit die obigen Kriterien mit soziodemographischen Merkmalen, Berufsorientierungen und persönlichem Involvement der Befragten zusammenhängen. Die statistische Analyse des ersten Teilmodells erfolgte mithilfe des Softwareprogramms SPSS 19.0. Im Folgenden werden die Ergebnisse der vier modellspezifischen Hypothesen dargestellt, ihre Diskussion erfolgt im Abschnitt 7.1.1. 


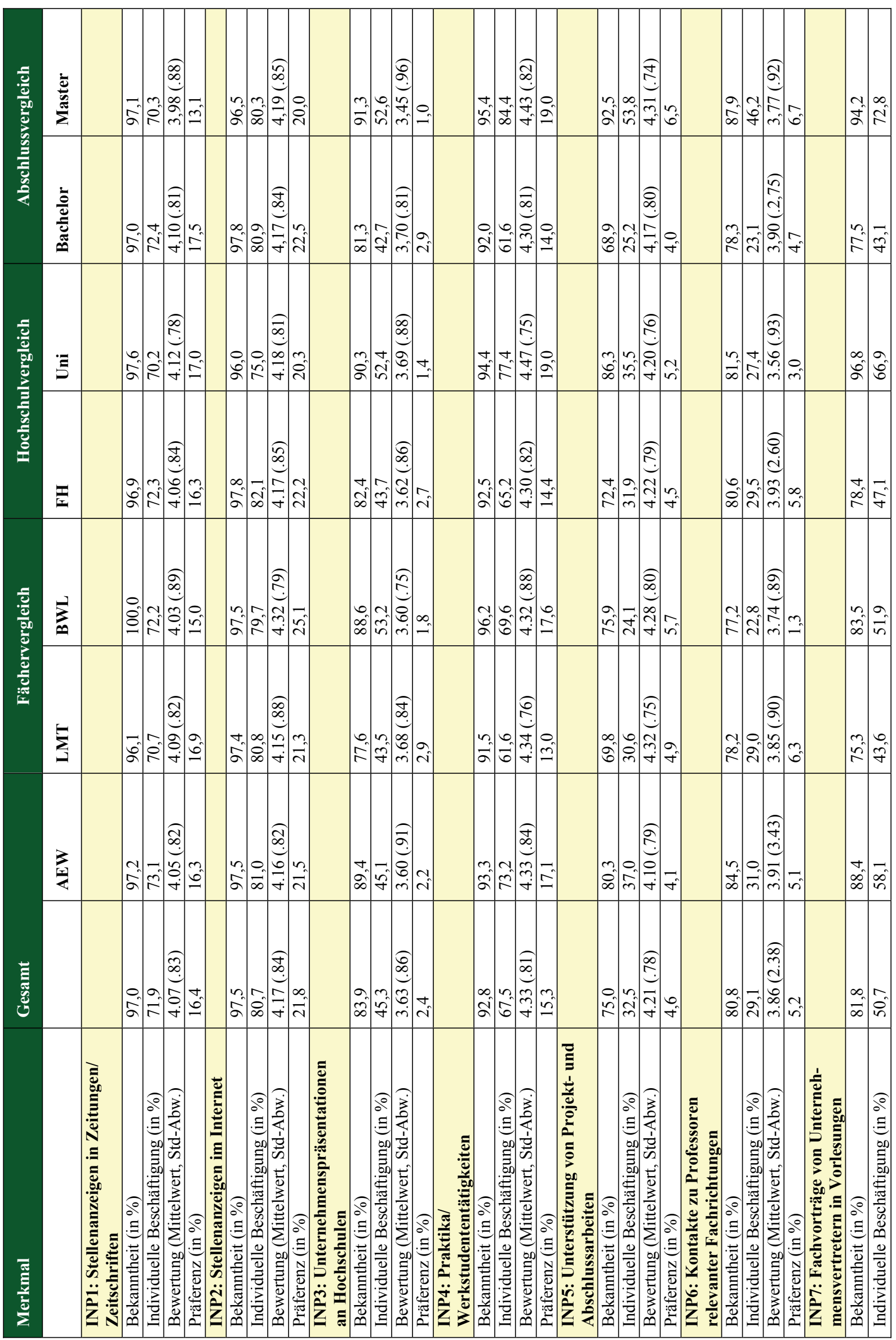




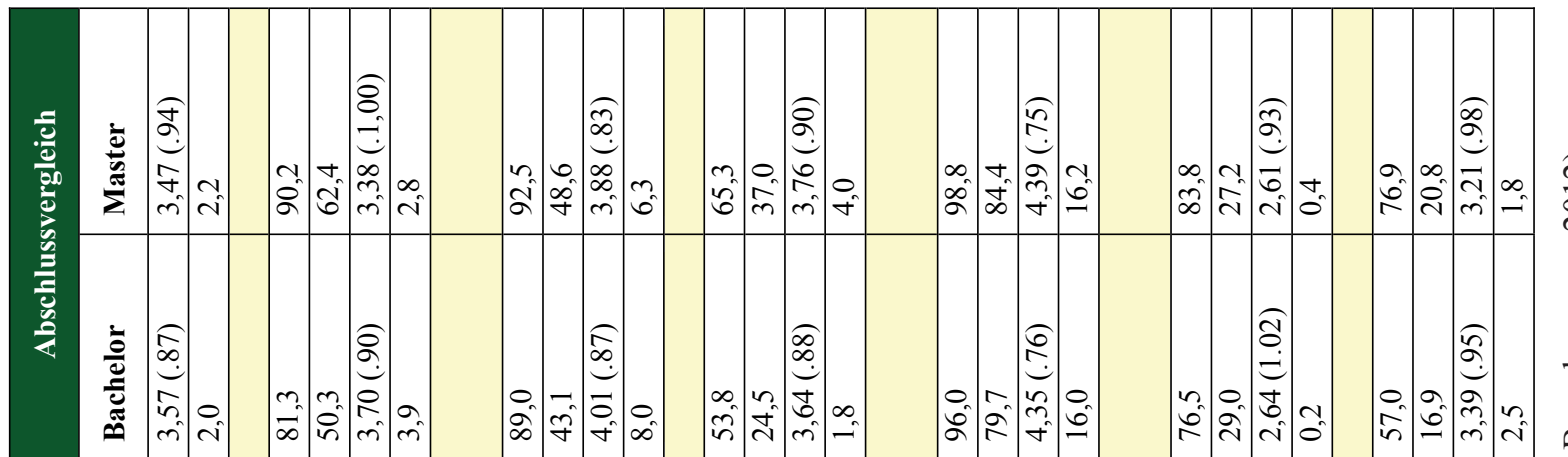

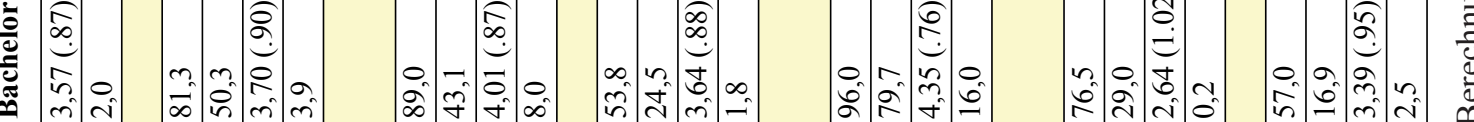

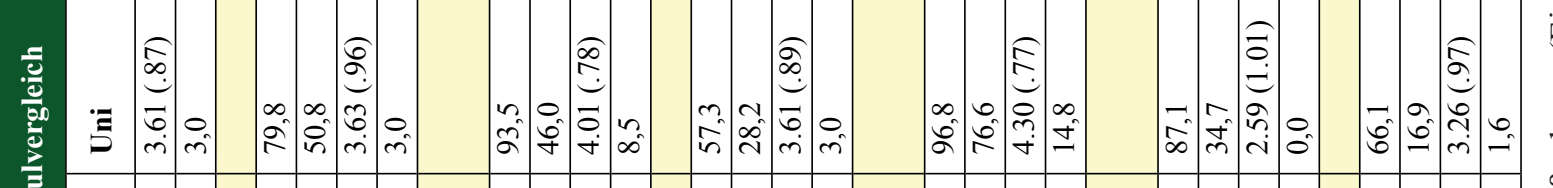

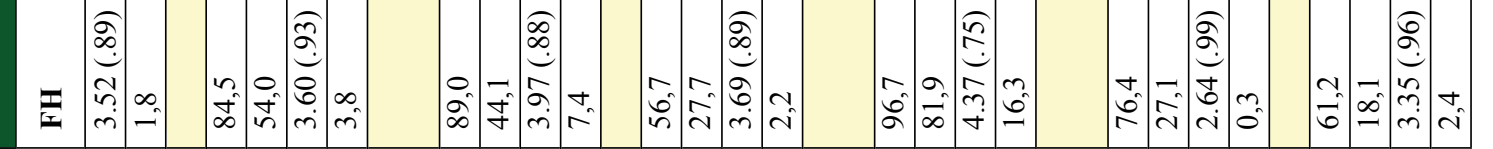

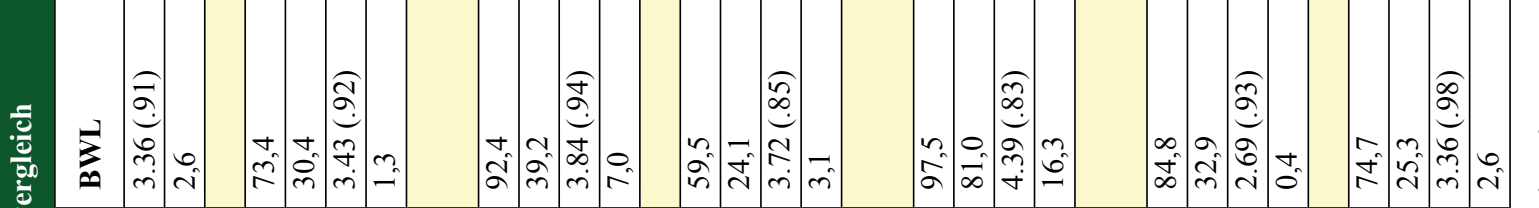

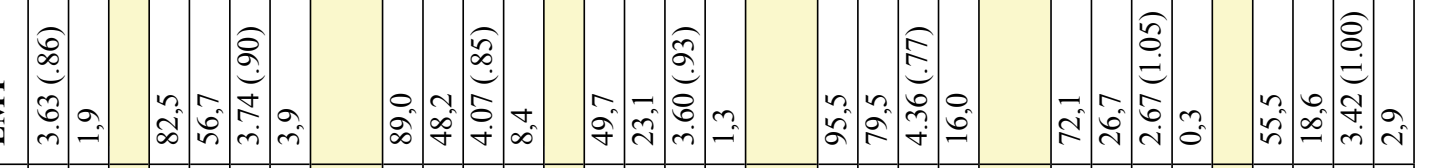

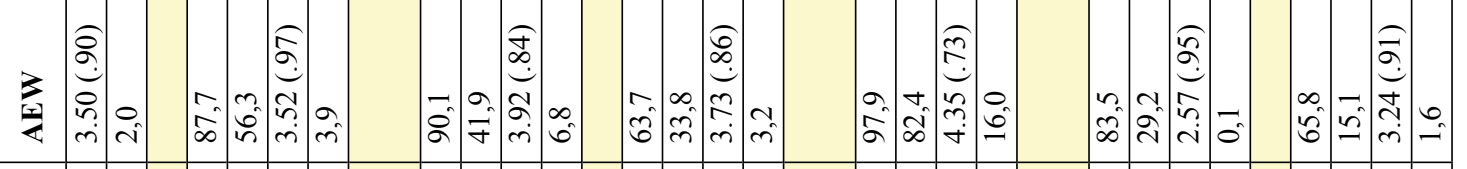

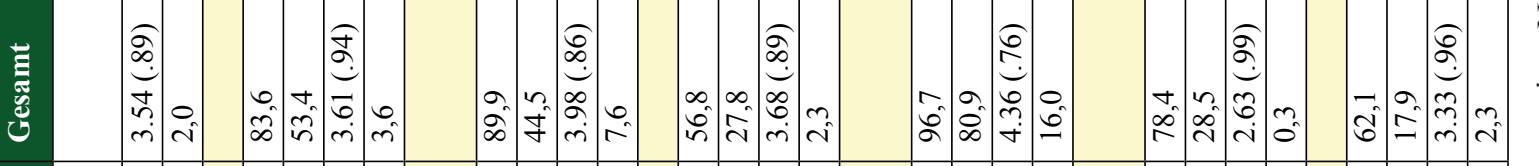

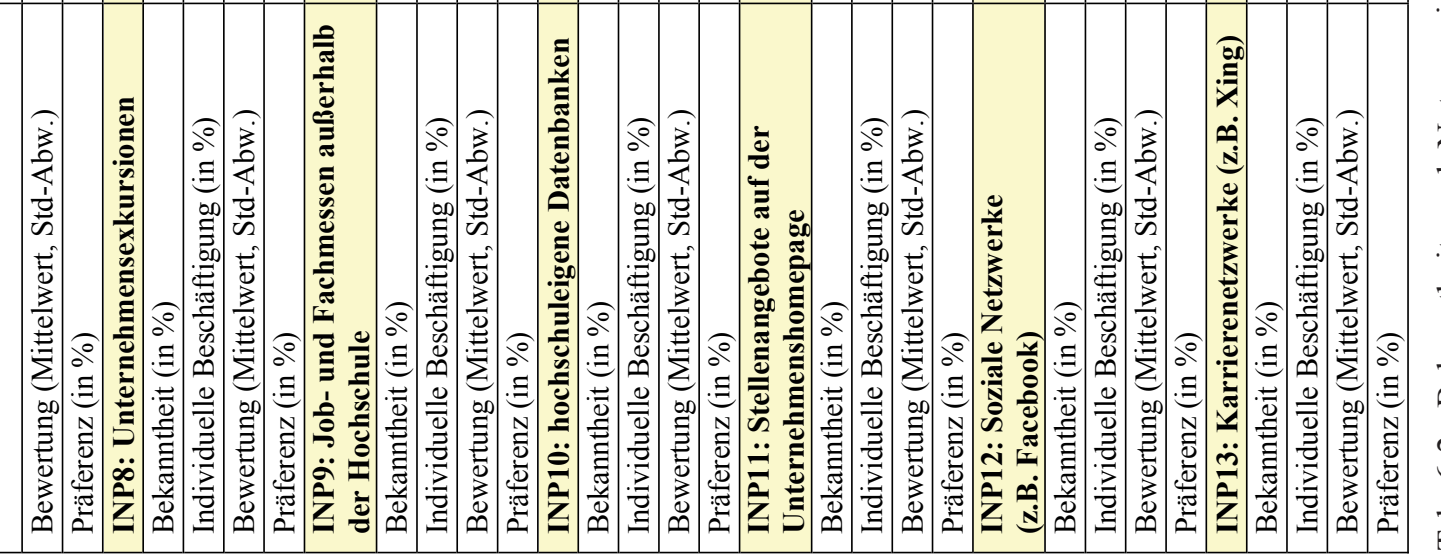




\subsubsection{Bekanntheitsgrad der Kommunikationsmaßnahmen}

Als Erstes wurde die Hypothese 1 überprüft, der zufolge der Bekanntheitsgrad einzelner Maßnahmen des Personalmarketings mit soziodemographischen und persönlichkeitsspezifischen Merkmalen der Befragten zusammenhängt. Wie Tabelle 6-2 zeigt, weisen die meisten Maßnahmen insgesamt einen hohen Bekanntheitsgrad auf. So gaben über 90 Prozent der Befragten an, Stellenanzeigen in Zeitschriften/Zeitungen sowie im Internet, Praktika/Werkstudententätigkeiten und Stellenangebote auf der Unternehmenshomepage zu kennen. Diesen folgen Unternehmenspräsentationen an Hochschulen, Kontakte zu Professoren relevanter Fachrichtungen, Fachvorträge von Unternehmensvertretern in Lehrveranstaltungen, Unternehmensexkursionen sowie Job-und Fachmessen außerhalb der Hochschule, die von über 80 Prozent der Befragten als bekannt angegeben wurden. Daran schließen sich mit einem über 70 Prozent liegenden Bekanntheitsgrad die Unterstützung Projekt- und Abschlussarbeiten sowie soziale Netzwerke (z.B. Facebook) an. Als weniger bekannt wurden hingegen hochschuleigene Datenbanken sowie Karrierenetzwerke (z.B. Xing) eingestuft, deren Bekanntheitsgrad bei 56,8 Prozent bzw. 62,1 Prozent liegt.

Da es sich beim Bekanntheitsgrad der Maßnahmen um eine nominal skalierte abhängige Variable mit den beiden Ausprägungen bekannt vs. nicht bekannt handelt, wurde als Analysemethode die binär-logistische Regression gewählt. Die logistische Regression unterscheidet sich insoweit von der linearen, als dass in der erstgenannten die Eintrittswahrscheinlichkeit der gewählten Ausprägung geschätzt wird und nicht die eigentliche Höhe der abhängigen Variablen (BACKHAUS et al., 2011). Gleichzeitig sind die Koeffizienten der logistischen Regression schwieriger zu interpretieren, da ein Vergleich der Koeffizienten aufgrund ihrer unterschiedlichen Ausprägung nicht möglich ist. Insofern beziehen sich die im Folgenden dargestellten Befunde ausschließlich darauf, in welche Richtung und wie stark die Bekanntheitswahrscheinlichkeit einzelner Maßnahmen durch die Prädiktoren beeinflusst wird. Während die Wirkungsrichtung anhand des Vorzeichens des Koeffizienten bestimmt wird, lässt sich die Stärke des Einflusses der unabhängigen Variablen aus der sog. Odds Ratio (OR) ablesen. Zudem ist zu beachten, dass die Interpretation der Prädiktoren stets in Bezug zur jeweiligen Referenzkategorie erfolgt. Mittels der Wald-Statistik wurden zudem die Signifikanzen der im Modell berücksichtigten Prädiktoren überprüft.

Als Prädiktoren wurden zum einen die soziodemographischen Variablen Geschlecht, Abschlussund Hochschulart, Fachrichtung sowie Anzahl bis zum Studienende Fachsemester betrachtet. Die soziodemographischen Variablen wurden um persönlichkeitsspezifische Variablen Berufsorientierung sowie persönliches Involvement ergänzt. Es sollte somit überprüft werden, ob der Bekanntheitsgrad der Maßnahmen durch diese unabhängigen Variablen, die zum Teil metrisch und zum Teil kategorial skaliert sind, vorhergesagt werden kann. Im Folgenden werden nur die Ergebnisse solcher Modelle dargestellt, in denen der Bekanntheitsgrad besser vorhergesagt werden konnte als in einem Modell ohne Prädiktoren. 


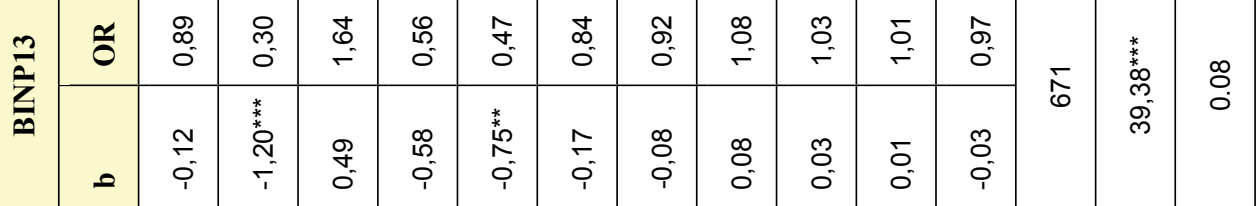

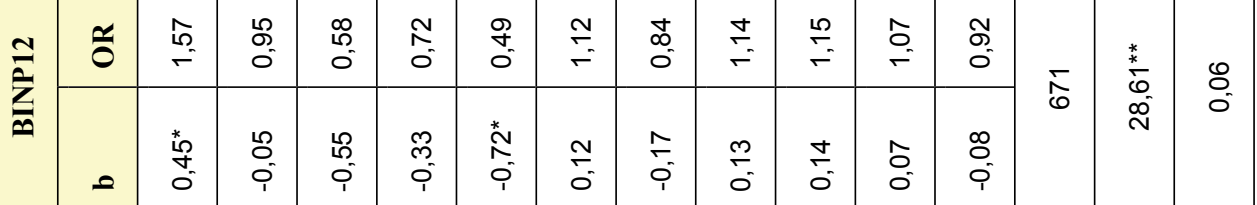

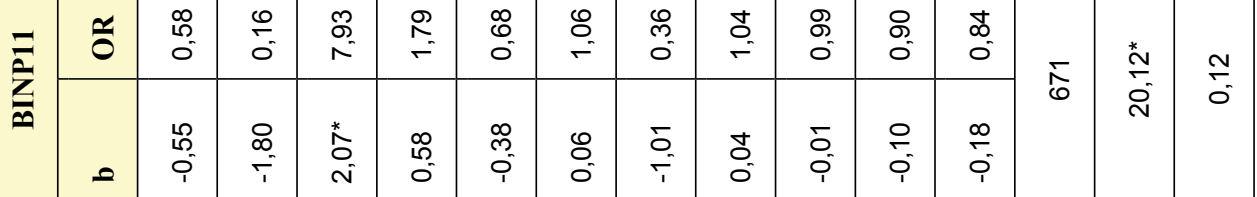

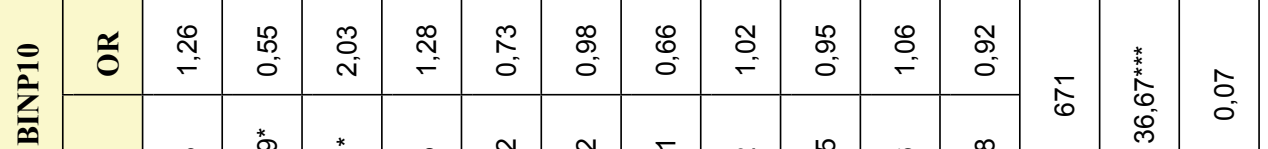

ฮิ่

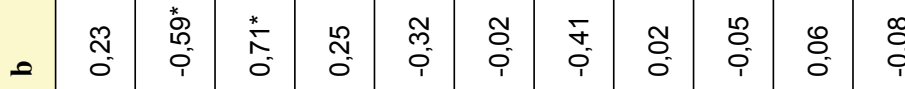

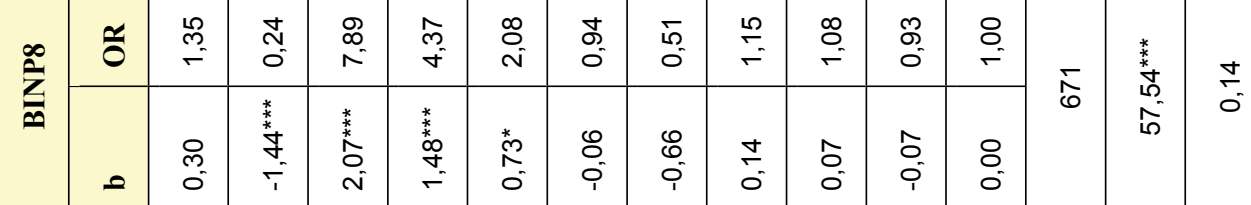

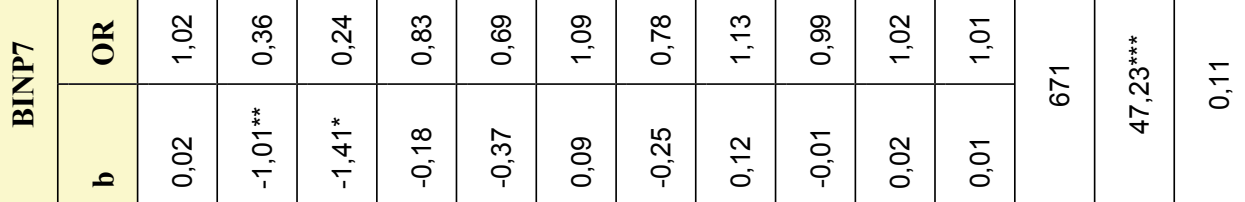

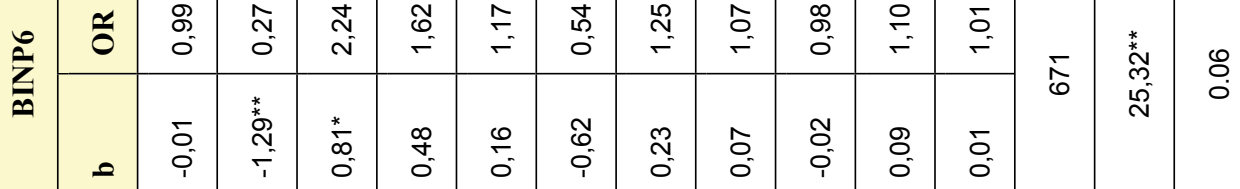

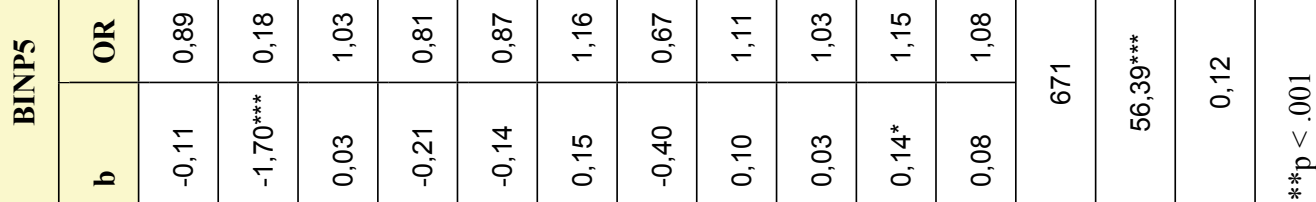

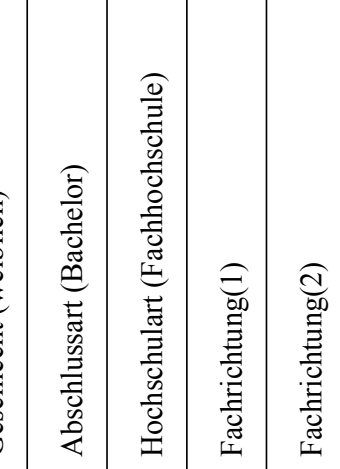

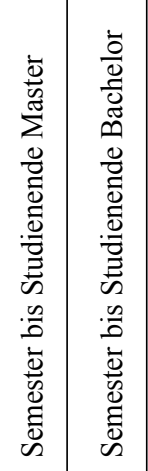

$\mid$

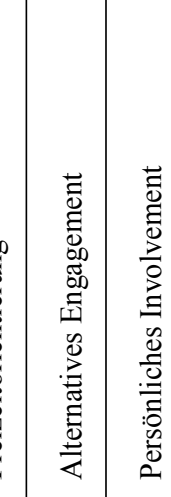


Aus den regressionsanalytischen Ergebnissen wird zunächst einmal ersichtlich, dass der Bekanntheitsgrad von sieben Maßnahmen anhand der soziodemographischen und persönlichkeitsspezifischen Variablen vorhergesagt werden konnte. So ergeben sich bei der Unterstützung von Projekt- und Abschlussarbeiten für die Prädiktoren Bachelorstudium $(b=-1.70$, $\left.S E_{b}=.36, O R=.18, \mathrm{p}<.001\right)$ und alternatives Engagement $\left(b=.14, S E_{b}=.07, O R=1.15\right.$, $\mathrm{p}<.05)$ statistisch signifikante Regressionsgewichte. Demnach ist die Wahrscheinlichkeit gröBer, dass Masterstudierende und alternativ engagierte Studierende dieses Instrument des Personalmarketings kennen. Für den Bekanntheitsgrad von Kontakten zu Professoren relevanter Fachrichtungen kann neben dem Bachelorstudium $\left(b=-1.29, S E_{b}=.43, O R=.28, \mathrm{p}<.01\right)$ die Fachhochschule $\left(b=.81, S E_{b}=.38, O R=2.24, \mathrm{p}<.05\right)$ als statistisch signifikanter Prädiktor identifiziert werden. Hier zeigt sich somit, dass Studierende an Fachhochschulen mit einer 2,2 Mal größeren Wahrscheinlichkeit diese Maßnahme kennen. Ebenso erweisen sich diese beiden Prädiktoren zur Vorhersage des Bekanntheitsgrades von Fachvorträgen von Unternehmensvertretern in Vorlesungen als statistisch signifikant: Sowohl bei Bachelorstudierenden $\left(b=-1.01, S E_{b}=.22, O R=.36, \mathrm{p}<.01\right)$ als auch bei Studierenden an Fachhochschulen liegt eine geringere Wahrscheinlichkeit vor, diese Maßnahme zu kennen $\left(b=-1.41, S E_{b}=.57\right.$, $O R=.24, \mathrm{p}<.05)$. Betrachtet man den Bekanntheitsgrad von Unternehmensexkursionen, so zeigt sich, dass bei Bachelorstudierenden eine geringere Bekanntheitswahrscheinlichkeit besteht als bei Masterstudierenden $\left(b=-1.44, S E_{b}=.42, O R=.24, \mathrm{p}<.001\right)$. Demgegenüber ist die Wahrscheinlichkeit, Unternehmensexkursionen zu kennen, bei Studierenden an Fachhochschulen 7,9 Mal größer als bei ihren Kommilitonen an Universitäten ( $b=2.07, S E_{b}=.46$, $O R=7.89, \mathrm{p}<.001)$. Zudem weist die Fachrichtung einen statistisch signifikanten Einfluss auf, und zwar gleichzeitig für beide Dummy-Variablen. Der signifikante Einfluss der DummyVariable Fachrichtung(1) bedeutet, dass gegenüber der Referenzgruppe der BWL-Studierenden bei AEW-Studierende eine größere Wahrscheinlichkeit vorliegt, Unternehmensexkursionen zu kennen $\left(b=1.48, S E_{b}=.44, O R=4.38, \mathrm{p}<.001\right)$. Aus der Odds Ratio lässt sich ableiten, dass diese Wahrscheinlichkeit für AEW-Studierende 4,4 Mal so groß ist, mit einem allerdings sehr breiten Konfidenzintervall von 1,9 bis 10,3. Zu beachten ist zugleich, dass die getroffene Aussage nur für die Betrachtung dieses Satzes von untereinander abhängigen Prädiktoren gilt. Die zweite Dummy-Variable Fachrichtung(2) zeigt ein ähnliches Muster: Die Bekanntheitswahrscheinlichkeit von Unternehmensexkursionen ist bei den LMT-Studierenden 2,1 Mal größer als bei BWL-Studierenden $\left(b=.73, S E_{b}=.31, O R=2.08, \mathrm{p}<.05\right)$. Zur Vorhersage des Bekanntheitsgrades hochschuleigener Datenbanken erweisen sich die soziodemographischen Variablen Bachelorstudium $\left(b=-.59, S E_{b}=.25, O R=.55, \mathrm{p}<.05\right)$ und Fachhochschule $(b=.71$, $\left.S E_{b}=.28, O R=2.03, \mathrm{p}<.05\right)$ als statistisch signifikante Prädiktoren. Bei den Stellenangeboten auf der Unternehmenshomepage trägt indes nur der Prädiktor Fachhochschule signifikant zur Vorhersage des Bekanntheitsgrades bei $\left(b=2.07, S E_{b}=.97, O R=7.93, \mathrm{p}<.05\right)$. Studierende an Fachhochschulen weisen demzufolge eine 7,9 Mal größere Wahrscheinlichkeit auf, diese Maßnahme zu kennen. Auch beim Bekanntheitsgrad sozialer Netzwerke erweist sich nur 
ein Prädiktor als statistisch signifikant: Bei LMT-Studierenden ist die entsprechende Wahrscheinlichkeit geringer als in der Referenzgruppe der BWL-Studierenden ( $b=-.72, S E_{b}=.34$, $O R=.49, \mathrm{p}<.05)$. Betrachtet man die Karrierenetzwerke, so zeigen die beiden Prädiktoren Bachelorstudium $\left(b=-1.20, S E_{b}=.27, O R=.30, \mathrm{p}<.001\right)$ und Fachrichtung $\left(b=-.75, S E_{b}=.29\right.$, $O R=.47, \mathrm{p}<.01)$ einen statistisch signifikanten Einfluss auf die Bekanntheitswahrscheinlichkeit. Demzufolge besteht bei LMT-Studierenden im Vergleich zu BWL-Studierenden eine geringere Wahrscheinlichkeit, Karrierenetzwerke zu kennen. Bei der Beurteilung der Modellgüte ist festzustellen, dass die $\mathrm{Chi}^{2}$-Werte auf einen signifikanten Einfluss der unabhängigen Variablen hinweisen. Allerdings wird die Stärke dieses Einflusses durch Nagelkerkes $\mathrm{R}^{2}$, das bei allen Modellen unter 0,2 liegt, deutlich relativiert. Insgesamt ist somit davon auszugehen, dass die aufgenommenen Variablen zwar einen signifikanten, aber zugleich einen ausgesprochen geringen Einfluss haben. Angesichts der eher explorativ angelegten Fragestellung sind diese Werte jedoch tolerierbar, da sie zumindest Tendenzen für relevante Einflussgrößen aufzeigen.

\subsubsection{Nutzungsintensität der Kommunikationsmaßnahmen}

Als Nächstes wurde die Hypothese 2 untersucht, in der angenommen wurde, dass die Nutzungsintensität einzelner Maßnahmen mit den soziodemographischen und persönlichkeitsspezifischen Merkmalen der Befragten zusammenhängt. Hierbei zeigt sich zunächst, dass die Nutzungsintensität teilweise deutlich unter dem Bekanntheitsgrad der einzelnen Maßnahmen liegt (vgl. Tab. 6-2). Demnach gehören zu den am meisten genutzten Maßnahmen mit über 70 Prozent Stellenanzeigen in Zeitungen/Zeitschriften und im Internet sowie Stellenangebote auf der Unternehmenshomepage. Wenig genutzt werden hingegen Karrierenetzwerke, soziale Netzwerke, hochschuleigene Datenbanken und Kontakte zu Professoren relevanter Fachrichtungen, bei denen die individuelle Nutzungsintensität unter 30 Prozent liegt. 


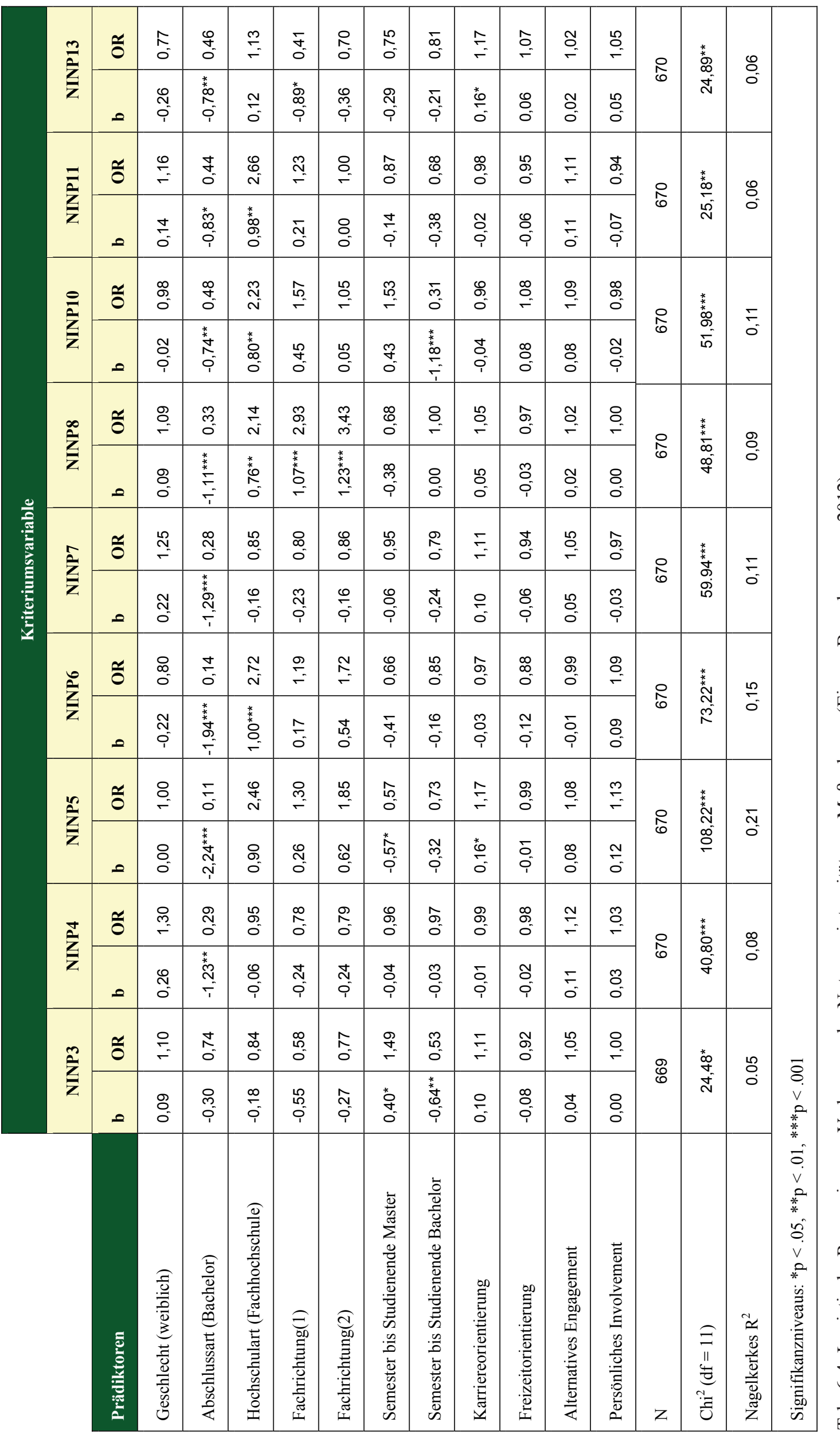


Das Vorgehen zur Analyse der Nutzungsintensität einzelner Instrumente des Personalmarketings gestaltet sich analog zur Analyse des Bekanntheitsgrades. Auch hier wurde die logistische Regression mit der Kriteriumsvariable Nutzungsintensität und den soziodemographischen und persönlichkeitsspezifischen Variablen als Prädiktoren berechnet. Daraus geht hervor, dass die regressionsanalytischen Ergebnisse überwiegend signifikant ausfallen. Bei den Unternehmenspräsentationen an Hochschulen wirkt sich die Anzahl der bis zum Studienende verbleibender Fachsemester sowohl im Master- als auch im Bachelorstudium signifikant auf die persönliche Teilnahme aus, wobei die Wirkungsrichtung unterschiedlich ist: Während die Nutzungswahrscheinlichkeit im Bachelorstudium erwartungskonform mit abnehmender Semesteranzahl steigt ( $\left.b=-.64, S E_{b}=.24, O R=.33, \mathrm{p}<.01\right)$, ist im Masterstudium aufgrund des positiven Regressionskoeffizienten ein gegenteiliges Muster zu beobachten $\left(b=.40, S E_{b}=.20, O R=1.49\right.$, $\mathrm{p}<.05)$. Bei der Nutzungswahrscheinlichkeit von Praktika/Werkstudententätigkeiten zeigt sich indes, dass diese bei Bachelorstudierenden im Vergleich zu Masterstudierenden geringer ausfällt $\left(b=-1.23, S E_{b}=.29, O R=.29, \mathrm{p}<.001\right)$. Demgegenüber ergeben sich für die Unterstützung von Projekt- und Abschlussarbeiten statistisch signifikante Regressionsgewichte für die Prädiktoren Bachelorstudium $\left(b=-2.23, S E_{b}=.28, O R=.11, \mathrm{p}<.001\right)$, Fachhochschule $\left(b=.89, S E_{b}=.30, O R=2.46, \mathrm{p}<.01\right)$ sowie bis zum Studienende verblebende Fachsemesteranzahl im Masterstudium $\left(b=-.57, S E_{b}=.22, O R=.57, \mathrm{p}<.05\right)$ und Karriereorientierung $\left(b=.16, S E_{b}=.07, O R=1.17, \mathrm{p}<.05\right)$. Beim Nutzungsniveau von Kontakten zu Professoren relevanter Fachrichtungen stellt sich neben dem Bachelorstudium $\left(b=-1.94, S E_{b}=.27\right.$, $O R=.14, \mathrm{p}<.001)$ die Fachhochschule $\left(b=1.00, S E_{b}=.31, O R=2.72, \mathrm{p}<.01\right)$ als statistisch signifikanter Prädiktor heraus. Während die Nutzungswahrscheinlichkeit bei Bachelorstudierenden im Vergleich zu Masterstudierenden geringer ausfällt, ist sie bei Studierenden an Fachhochschulen 2,7 Mal höher als bei ihren Kommilitonen an Universitäten. Demgegenüber erweist sich bei der Vorhersage der Nutzungsintensität das Bachelorstudium als alleiniger statistisch signifikanter Prädiktor $\left(b=-1.29, S E_{b}=.25, O R=.28, \mathrm{p}<.001\right)$. Anders verhält es sich mit der Nutzungsintensität von Unternehmensexkursionen: Neben Bachelorstudium $\left(b=-1.11, S E_{b}=.26, O R=.33, \mathrm{p}<.001\right)$ und Fachhochschule $\left(b=.76, S E_{b}=.28, O R=2.14\right.$, $\mathrm{p}<.01$ ), weist auch die Fachrichtung einen für beide Dummy-Variablen geltenden Einfluss auf. Demzufolge ist die Nutzungswahrscheinlichkeit von Unternehmensexkursionen bei AEWStudierenden um 2,9 Mal $\left(b=1.08, S E_{b}=.31, O R=2.93, \mathrm{p}<.001\right)$ und bei LMT-Studierenden um 3,4 Mal $\left(b=1.23, S E_{b}=.28, O R=3.43, \mathrm{p}<.001\right)$ größer als in der Referenzgruppe der BWL-Studierenden. Bei der Nutzungsintensität hochschuleigener Datenbanken resultieren für die Prädiktoren Bachelorstudium $\left(b=-.74, S E_{b}=.26, O R=.48, \mathrm{p}<.01\right)$, Fachhochschule $(b=$ $\left..80, S E_{b}=.30, O R=2.23, \mathrm{p}<.01\right)$ sowie bis zum Studienende verbleibende Fachsemesteranzahl im Bachelorstudium $\left(b=-1.12, S E_{b}=.27, O R=.31, \mathrm{p}<.001\right)$ statistisch signifikante Koeffizienten. Im Fall sozialer Netzwerke zeigen die Prädiktoren Bachelorstudium ( $b=-.83, S E_{b}=.34$, $O R=.44, \mathrm{p}<.05)$ und Fachhochschule $\left(b=.98, S E_{b}=.37, O R=2.66, \mathrm{p}<.01\right)$ einen signifikanten Einfluss auf die Nutzungswahrscheinlichkeit. Zur Vorhersage der Nutzungsintensität von Karrierenetzwerken erweisen sich schließlich die Prädiktoren Bachelorstudium $(b=-.78$, 
$\left.S E_{b}=.29, O R=.46, \mathrm{p}<.01\right)$, Fachrichtung $(1)\left(b=-.89, S E_{b}=.37, O R=.41, \mathrm{p}<.05\right)$ und Karriereorientierung $\left(b=.16, S E_{b}=.08, O R=1.17, \mathrm{p}<.01\right)$ als statistisch signifikante Prädiktoren. Demzufolge fällt die Nutzungswahrscheinlichkeit von Karrierenetzwerken bei AEW-Studierenden geringer aus als in der Referenzgruppe der BWL-Studierenden. Analog zum obigen Regressionsmodell bleibt hinsichtlich der Modellgüte festzuhalten, dass die berücksichtigten Prädiktoren sich nur sehr mäßig auf die Nützlichkeitsintensität auswirken.

\subsubsection{Nützlichkeitsbewertung der Kommunikationsmaßnahmen}

Um verzerrte Ergebnisse bei der Nützlichkeitsbewertung der Maßnahmen zu vermeiden, wurden in die weiterführenden Analysen ausschließlich solche Maßnahmen einbezogen, die den Befragten bekannt waren. Die Mittelwerte liegen mit Ausnahme sozialer Netzwerke über der mittleren Antwortkategorie und weisen somit auf eine insgesamt positive Nützlichkeitsbewertung hin. Zur explorativen Beantwortung der Frage, wie viel an Varianz soziodemographische und persönlichkeitsspezifische Variablen im metrisch skalierten Kriterium Nützlichkeitsbewertung aufklären, wurde für jede Maßnahme eine multiple Regression durchgeführt. Um ergänzend zu überprüfen, ob sich die prognostische Validität bei der Nützlichkeitsbewertung erhöht, wenn zusätzlich zu den soziodemographischen auch persönlichkeitsspezifische Variablen als Prädiktoren eingesetzt werden, wurde auf eine blockweise schrittweise Regression zurückgegriffen. Dabei gingen im ersten Schritt die soziodemographischen Variablen Hochschulart, Fachrichtung und Geschlecht sowie die Anzahl bis zum Studienende verbleibender Fachsemester ein, im zweiten Schritt wurden entsprechend die Berufsorientierungen und persönliches Involvement der Befragten in das Regressionsmodell aufgenommen. Da es sich bei den vier zuerst genannten Prädiktoren um kategoriale Variablen handelt, wurden sie als Dummy-Variablen kodiert. Die metrische Variable bis zum Studienende verbleibende Fachsemesteranzahl wurde zudem zentriert. Ferner wurde angenommen, dass sich die verbleibende Fachsemesteranzahl im Bachelorstudium anders auswirkt als im Masterstudium, da die Masterstudierenden bereits ein Bachelorstudium absolviert haben und somit mehr bzw. andere Erfahrungen im Umgang mit Informationsquellen aufweisen könnten. Dies wurde durch einen Interaktionseffekt modelliert. Die Variable aktuelle Fachsemesteranzahl wurde hingegen aufgrund von Multikollinearität nicht in das Regressionsmodell aufgenommen. Als Kriterienvariablen fungierten die Nützlichkeitsbewertungen der Maßnahmen des Personalmarketings.

Vorab wurde bei sämtlichen Regressionsmodellen überprüft, ob die Voraussetzungen einer multiplen Regression erfüllt sind (BACKHAUS et al., 2011): 
1. Für alle Personen gilt die gleiche lineare Gleichung (Linearität). Dies wurde aufgrund der theoretisch fundierten Auswahl von Prädiktoren einerseits und des als linear postulierten Zusammenhanges der unabhängigen und abhängigen Variablen andererseits als erfüllt angesehen.

2. Für alle Kombinationen von Prädiktorwerten haben die Residuen die gleiche Varianz (Varianzhomogenität $=$ Homoskedastizität). Diese Voraussetzung wurde mittels eines Diagramms überprüft, auf dem die geschätzten Werte gegen die Residuen abgebildet wurden. Heteroskedastizität hätte bei einem erkennbaren Zusammenhang vorgelegen. Bei den unten dargestellten Regressionsanalysen war dies jedoch nicht der Fall, sodass auch diese Annahme als erfüllt angesehen wurde.

3. Die Residuen sind statistisch unabhängig voneinander, andernfalls liegt eine Autokorrelation vor. Die Frage, ob eine Autokorrelation vorliegt, wurde mittels des Durbin-WatsonTests überprüft. Da die entsprechenden Werte nur wenig von dem Wert 2 abwichen, lag hier keine Autokorrelation vor.

4. Es liegt keine Multikollinearität der Prädiktoren vor. Multikollinearität hätte dann vorgelegen, wenn eine unabhängige Variable als lineare Kombination einer anderen Variablen hervorgeht. Innerhalb der Regressionsanalyse lässt sich die Annahme anhand von zwei Statistiken überprüfen: dem sog. Toleranzwert und dem Variance Inflation Factor (VIF). Als Faustregel wird empfohlen, dass der Toleranzwert nicht weniger als 0,25 und der VIF nicht mehr 5 betragen sollte (URBAN und MAYERL, 2011). Sämtliche in den Regressionsanalysen resultierenden Toleranz- bzw. VIF-Werte verletzen diese Kriterien nicht.

5. Die Residuen sind multivariat normalverteilt. Diese Voraussetzung wurde anhand eines Histogramms der Residuen überprüft. Im Ergebnis zeigte sich, dass die optisch feststellbare Anpassung der empirischen Verteilung an die theoretische Normalverteilung recht gut war. Bei der Interpretation wurde zugleich berücksichtigt, dass keine empirische Verteilung exakt der theoretischen Normalverteilung folgt und bei großen Stichproben - wie im vorliegenden Fall - selbst „unbedeutende“ Abweichungen zu statistischer Signifikanz führen. Dementsprechend konnte in mehreren regressionsanalytischen Studien nachgewiesen werden, dass trotz nicht erfüllter Normalverteilungsannahmen vor allem bei großen Stichproben valide Ergebnisse zu erwarten sind (BORG und STAUFENBIEL, 2007).

Nachfolgend werden nur solche Modelle ausführlich behandelt, in denen der multiple Korrelationskoeffizient signifikant von null verschieden und somit nicht zufällig, sondern durch den Zusammenhang der Daten entstanden war. 


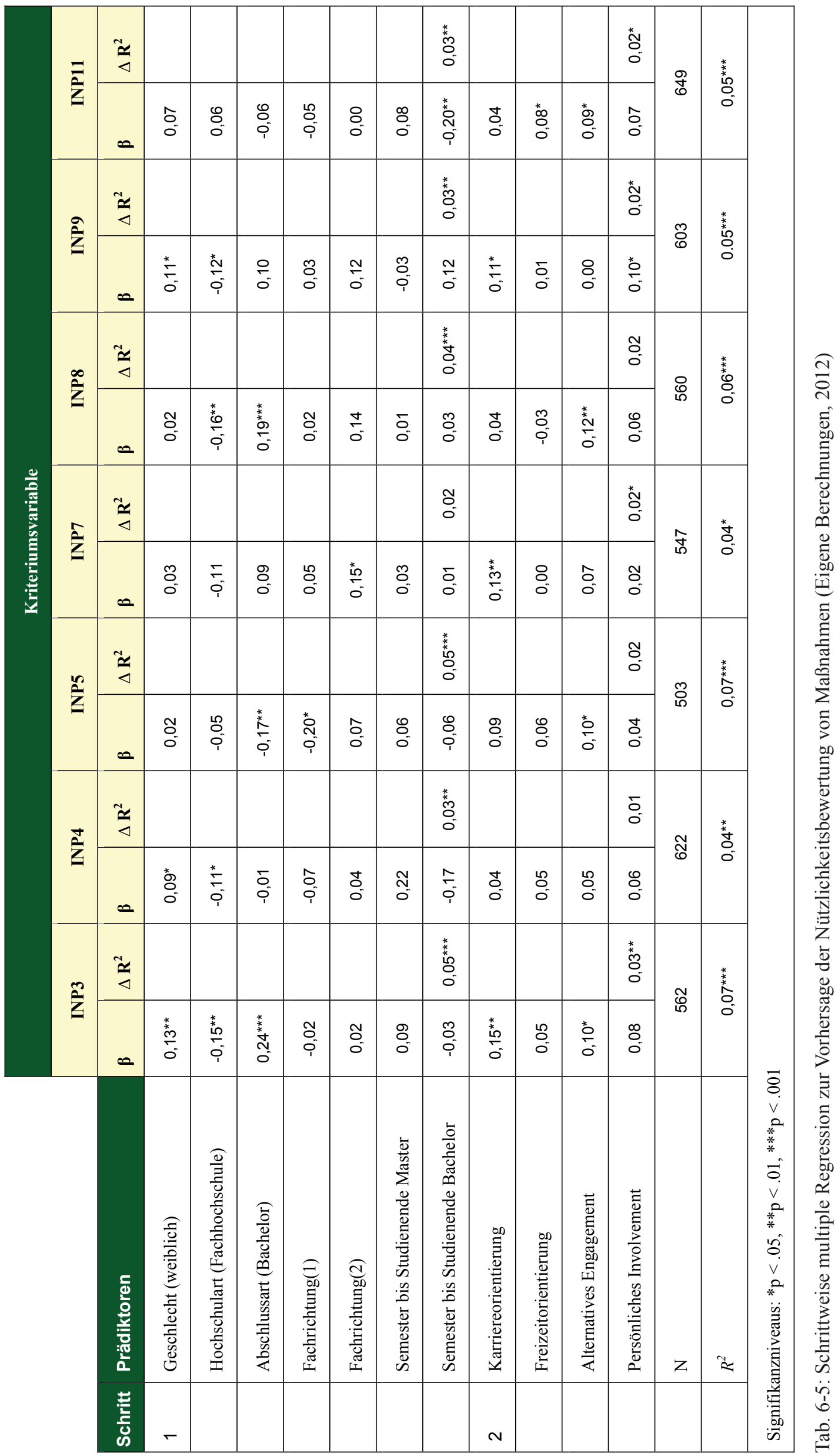


Wie Tabelle 6-5 zeigt, kann die Nützlichkeitsbewertung von sieben der insgesamt dreizehn Maßnahmen signifikant anhand der oben genannten Prädiktoren vorhergesagt werden. Demnach werden Unternehmenspräsentationen an Hochschulen von weiblichen Studierenden im Vergleich zu männlichen $(\beta=.13, \mathrm{p}<.01)$ und von Bachelorstudierenden vergleichen mit Masterstudierenden $(\beta=.24, \mathrm{p}<.001)$ als nützlicher beurteilt, während Studierende an Fachhochschulen diese weniger sinnvoll bewerten als Studierende an Universitäten $(\beta=-.15$, $\mathrm{p}<.01)$. Bei dieser Maßnahme leisten zudem die beiden persönlichkeitsspezifischen Variablen Karriereorientierung $(\beta=.15, \mathrm{p}<.01)$ und alternatives Engagement $(\beta=.10, \mathrm{p}<.05)$ einen statistisch signifikanten zusätzlichen Beitrag zur Vorhersage der Nützlichkeitsbewertung. Zur Vorhersage von Praktika/Werkstudententätigkeiten wirken sich die beiden soziodemographischen Variablen Geschlecht $(\beta=.09, \mathrm{p}<.05)$ und die Hochschulart $(\beta=-.11, \mathrm{p}<.05)$ in gleicher Weise wie oben signifikant zur Vorhersage der Nützlichkeitsbewertung aus. Anders als erwartet, scheint die Nützlichkeitsbewertung jedoch zu Beginn des Studiums größer zu sein als am Ende $(\beta=.22, \mathrm{p}<.01)$, wobei sich der positive Koeffizient ausschließlich auf die Masterstudierenden bezieht. Bei den Bachelorstudierenden zeigt sich indes ein erwartungskonformes Ergebnis: Im Bachelorstudium steigt die Nützlichkeitsbewertung von Praktika/Werkstudententätigkeiten mit abnehmender Anzahl bis zum Studienende verbleibender Semester $(\beta=-.17, \mathrm{p}<.05)$. Demgegenüber bewerten Bachelorstudierende die Nützlichkeit von Projekt- und Abschlussarbeiten negativer als Masterstudierende $(\beta=-.17, \mathrm{p}<.01)$, ebenso schätzen AEW-Studierende im Vergleich zu BWL-Studierenden diese Maßnahme als weniger sinnvoll ein $(\beta=-.20$, $\mathrm{p}<.05$ ). Darüber hinaus weist das alternative Engagement eine inkrementelle Validität auf $(\beta=.10, \mathrm{p}<.05)$. Fachvorträge von Unternehmensvertretern in Vorlesungen werden indes von LMT-Studierenden deutlich besser beurteilt als von BWL-Studierenden $(\beta=.16, \mathrm{p}<.05)$. Ferner fungiert die Karriereorientierung als signifikanter Prädiktor $(\beta=.13, \mathrm{p}<.01)$. Hinsichtlich ihrer Nützlichkeit bewerten Bachelorstudierende Unternehmensexkursionen positiver als Masterstudierende $(\beta=.19, \mathrm{p}<.001)$, während Studierende an Fachhochschulen im Vergleich zu Universitätsstudierenden eine negativere Einschätzung vornehmen $(\beta=-.16, p<.01)$. Hinzu kommt das alternative Engagement, das zusätzlich zu den soziodemographischen Variablen Varianz in der Nützlichkeitsbewertung dieser Maßnahmen aufklärt $(\beta=.12, \mathrm{p}<.01)$. Weiterhin messen weibliche Studierende Jobmessen außerhalb von Hochschulen einen höheren Stellenwert zu als männliche $(\beta=.11, \mathrm{p}<.05)$, während Studierende an Fachhochschulen verglichen mit Universitätsstudierenden diese als weniger informativ beurteilen $(\beta=-.12, \mathrm{p}<.05)$. Einen inkrementellen Beitrag leisten ferner die beiden persönlichkeitsspezifischen Variablen Karriereorientierung $(\beta=.11, \mathrm{p}<.05)$ und persönliches Involvement $(\beta=.10, \mathrm{p}<.05)$. Zur Vorhersage der Nützlichkeitsbewertung von Stellenangeboten auf der Unternehmenshomepage dient in erwartungskonformer Weise die bis zum Studienende verbleibende Fachsemesteranzahl, wobei dieser Koeffizient auf die Bachelorstudierenden zu beschränken ist $(\beta=-.20, \mathrm{p}<.01)$. Weitere statistisch signifikante Prädiktoren sind bei dieser Maßnahme die beiden Berufsorientierungen Freizeitorientierung $(\beta=.08, \mathrm{p}<.05)$ sowie alternatives Engagement $(\beta=.09, \mathrm{p}<.05)$. 
Um abschließend Aussagen über die Effektstärke der berechneten Regressionsmodelle zu treffen, wird das Bestimmtheitsmaß $\mathrm{R}^{2}$ herangezogen, das den aufgeklärten Varianzanteil wiedergibt. Gemäß der Klassifikation von COHEN (1988) zeigt sich hierbei, dass durchweg kleine Effektstärken zu verzeichnen sind und somit lediglich vier bis sieben Prozent der Varianz durch das aufgestellte Modell erklärt werden können.

\subsubsection{Präferenzmaß der Kommunikationsmaßnahmen}

Die Häufigkeitsverteilung des Bekanntheits- und Nutzungsgrades wurde um ein Präferenzmaß ergänzt, bei dem die Befragten anhand eines offenen Antwortformates die aus ihrer Sicht wichtigsten Maßnahmen nennen sollten. Hieraus wurde ein Präferenzmaß ermittelt, das sich aus dem Anteil derjenigen Maßnahmen zusammensetzt, die zu den drei bedeutendsten gehörten. $\mathrm{Zu}$ den am meisten präferierten Maßnahmen zählen demzufolge Stellenanzeigen in Zeitungen/ Zeitschriften und im Internet sowie Praktika/Werkstudententätigkeiten und Stellenangebote auf der Unternehmenshomepage (vgl. Tab. 6-2). Eine geringe Präferenz mit weniger als einem Prozent weisen hingegen soziale Netzwerke (z.B. Facebook) auf.

\begin{tabular}{|l|c|c|c|c|}
\hline Prädiktoren & df & N & Chi $^{2}$ & Phi/Cramers V \\
\hline Geschlecht & 12 & 1964 & 13,08 & 0,08 \\
\hline Abschlussart & 12 & 1964 & $37,63 * * *$ & 0,14 \\
\hline Hochschulart & 12 & 1964 & 16,34 & 0,09 \\
\hline Fachrichtung & 24 & 1964 & $36,57 *$ & 0,10 \\
\hline Signifikanzniveaus: $* \mathrm{p}<.05, * * * \mathrm{p}<.001$ & & \\
\hline
\end{tabular}

Tab. 6-6: Zusammenhänge von soziodemographischen Merkmalen mit dem Präferenzmaß von Maßnahmen (Eigene Berechnungen, 2012)

Im nächsten Schritt wurde der Zusammenhang zwischen Maßnahmenpräferenz und soziodemographischen Variablen untersucht. Während sich die Beziehung zwischen Hochschulart bzw. Geschlecht und Präferenzmaß als statistisch nicht signifikant erweist, zeigt sich für die beiden Variablen Fachrichtung bzw. Abschlussart ein statistisch signifikanter Zusammenhang mit dem Präferenzmaß (Abschlussart: $\chi_{(12, \mathrm{~N}=1964)}^{2}=37,63, \mathrm{p}<.001$; Fachrichtung: $\chi_{(24, \mathrm{~N}=1964)}^{2}=36,57, \mathrm{p}$ $<.05)$. Allerdings lässt sich der $\mathrm{Chi}^{2}$-Wert bei den Kreuztabellen nur schwer interpretieren, da dieser sowohl von $\mathrm{N}$ als auch von der Anzahl der Variablenausprägungen abhängt (AKREMI und BAUR, 2011). Die Stärke des Zusammenhangs wurde deshalb anhand der beiden Maße Phi (bei dichotom skalierten unabhängigen Variablen) bzw. Cramers V (bei unabhängigen Variablen mit mehr als zwei Ausprägungen) bestimmt. In Anbetracht der zu interpretierenden Werte (Phi bzw. Cramers V) von unter 0,30 ist die Stärke der Beziehung jedoch sowohl für die Abschlussart als auch für die Fachrichtung als ausgesprochen gering zu werten. 


\subsection{Analyse des zweiten Teilmodells: Differenzielle Wirkung des Arbeitgeberimages}

In den Discrete-Choice-Modellen wird grundsätzlich der Zusammenhang zwischen einer binär abhängigen Variablen in Form einer diskreten Auswahlentscheidung und einer oder mehreren metrisch und/oder kategorial skalierten unabhängigen Variablen untersucht (WEIBER und MÜHLHAUS, 2010). Dabei wird anhand der beobachteten Daten die Wahrscheinlichkeit bestimmt, dass ein Individuum n eine bestimmte Alternative i aus einer begrenzten Menge C (steht für: Choice-Sets) von Alternativen wählt. Die Auswahlwahrscheinlichkeit einer Alternative durch ein Individuum $\mathrm{P}_{\mathrm{n}}$ (i) basiert auf der Nutzenmaximierungsannahme der Zufallsnutzentheorie, d.h. es wird die Alternative mit dem größten individuellen Nutzen gewählt (LOUVIERE et al., 2000). Mit der nutzenmaximierenden Verhaltensannahme lassen sich die Discrete-ChoiceModelle zugleich von der logistischen Regression abgrenzen, da eine vergleichbare Annahme dort nicht vorhanden ist (TEMME, 2009).

Allerdings ist der Nutzen $U_{i}$ eines Entscheidungsträgers als latente Variable nicht direkt beobachtbar, sodass hierbei Wahrscheinlichkeitsaussagen über das Entscheidungsverhalten der Befragten getroffen werden müssen. Ursächlich hierfür sind folgende Aspekte (MAIER und WEISS, 1990, zit. in ENNEKING, 2003):

1. Wesentliche sozioökonomischen Variablen können nicht in ihrer Gesamtheit erfasst werden. So dürfte die Arbeitgeberwahlentscheidung von Befragten, die eine starke Nachfrage auf dem Arbeitsmarkt wahrnehmen, anders ausfallen als bei potenziellen Bewerbern, die ihre Arbeitsmarktchancen als eher negativ einschätzen. Infolgedessen können zwei Befragte, die sich anhand der beobachteten Größen nicht unterscheiden, aufgrund dieser Einschätzung bzw. Erfahrung zu unterschiedlichen Wahlentscheidungen gelangen.

2. Relevante persönlichkeitsspezifische Merkmale, wie beispielsweise Einstellungen oder Werthaltungen, lassen sich aufgrund unvollkommener Instrument-Variablen weder direkt noch indirekt messen.

3. Neben soziökonomischen und persönlichkeitsspezifischen Merkmalen erweisen sich auch die unbeobachteten Produktalternativen als bedeutsame Einflussfaktoren auf die Wahlentscheidung. Dies liegt darin begründet, dass es im Forschungskontext äußerst schwierig ist, sämtliche relevanten Produkteigenschaften ausreichend in einem Wahlexperiment zu berücksichtigen. So lassen sich beispielsweise nicht alle möglichen Eigenschaften des Produktes Stellenangebot darstellen, wodurch a priori ein gewisser Informationsverlust in Kauf genommen werden muss. 
Die Operationalisierung des Nutzens ist zwar grundsätzlich über beobachtbare Größen, zu denen Eigenschaften und Merkmale der Alternativen und Entscheidungsträger zählen, möglich. Allerdings kann der Nutzen auf der Datengrundlage der beobachtbaren Größen nur zu einem gewissen Teil erklärt werden, was eine Zerlegung der latenten Variablen in eine deterministische Komponente Vi und eine stochastische Komponente ei erfordert (URBAN, 1993; ENNEKING, 2003):

$$
\mathrm{U}_{\mathrm{i}}=\mathrm{V}_{\mathrm{i}}+\mathrm{e}_{\mathrm{i}}
$$

Gemäß der Nutzenmaximierungsannahme wählt ein Entscheidungsträger $\mathrm{i}$ die Alternative $\mathrm{j}$ dann aus, wenn der Nutzen Ui dieser Alternative höher ist als der Nutzen der übrigen Wahlalternativen:

$$
U_{j^{\prime} i}>U_{j i} \text { für } j=1 \ldots J
$$

Wird der Nutzen Ui in seine deterministische und einen stochastische Bestandteile zerlegt, so resultiert:

$$
\mathrm{V}_{\mathrm{j}^{\prime} \mathrm{i}}+\mathrm{e}_{\mathrm{j}^{\prime} \mathrm{i}}>\mathrm{V}_{\mathrm{ji}}+\mathrm{e}_{\mathrm{ji}}
$$

oder

$$
\mathrm{V}_{\mathrm{j}^{\prime} \mathrm{I}}-\mathrm{V}_{\mathrm{ji}}>\mathrm{e}_{\mathrm{ji}}-\mathrm{e}_{\mathrm{j}^{\prime} \mathrm{i}}
$$

Aus einer wahrscheinlichkeitstheoretischen Umformulierung ergibt sich die Wahlwahrscheinlichkeit einer Alternative $\mathrm{j}$ ”:

$$
P_{j{ }^{\prime} \mathrm{i}}=\operatorname{Prob}\left(\mathrm{V}_{\mathrm{j}^{\mathrm{i} i}}-\mathrm{V}_{\mathrm{ji}}>\mathrm{e}_{\mathrm{ji}}-\mathrm{e}_{\mathrm{j \textrm {i }}}\right) \text { für } \mathrm{j}=1 \ldots \mathrm{J} \backslash \mathrm{j}^{\mathrm{c}}
$$

Gemäß Gleichung (7) wählt der Entscheidungsträger i die Alternative j', wenn der beobachtbare Nutzen dieser Alternative größer ist als der Nutzen jeder anderen Alternative und diese Nutzendifferenz zugleich nicht durch die stochastische Größe $e_{j i}-e_{j{ }^{i}}$ überlagert wird.

Zur empirischen Umsetzung dieses Modells muss die aus beobachtbaren Größen bestehende deterministische Komponente $\mathrm{V}_{\mathrm{ji}}$ als eine Funktion des Parametervektors $\mathrm{X}_{\mathrm{ji}}$ dargestellt werden, der seinerseits einen substanziellen Beitrag zur Erklärung individueller Unterschiede im Wahlverhalten der Entscheidungsträger leistet (URBAN, 1993). In der Regel wird hierbei ein linearer Zusammenhang unterstellt mit $\beta_{\mathrm{i}}$ als Parametervektor und $\mathrm{X}_{\mathrm{ji}}$ stellvertretend für beobachtbare Merkmale der Alternativen $\mathrm{j}$ und Entscheidungsträger i, worunter im Einzelnen folgende Variablen fallen (ENNEKING, 2003): 
1. Sozioökonomische Variablen, die über die Befragten $n$, jedoch nicht über die Alternativen i variieren.

2. Generische Variablen, die sowohl über die Befragten $n$ als auch über die Alternativen $\mathrm{i}$ variieren.

3. Alternativenspezifische Variablen, die nur über eine Alternativ i variieren und für alle übrigen Alternativen den Wert null annehmen.

Um die Parameterschätzung mithilfe der Maximum-Likelihood-Schätzung (ML) vorzunehmen, muss die dazugehörige stochastische Größe $e$ über geeignete Verteilungsannahmen modelliert werden. Dabei wird das als Standard geltende multinomiale Logit-Modell (MNL) über eine Extremverteilung der Störterme beschrieben (TEMME, 2009). Aus der Nutzenmaximierungsannahme und der Annahme extremwertverteilter Störterme resultiert die klassische Form des MNL-Modells:

$$
P_{j i}=\frac{e^{X j i \beta}}{\sum_{j} e^{X j i \beta}}
$$

$\mathrm{P}_{\mathrm{ji}}$ gibt die Wahlwahrscheinlichkeit in Abhängigkeit der beobachteten Merkmale an. Somit entspricht die Wahlwahrscheinlichkeit einer Alternative dem Nutzen einer Alternative im Verhältnis zum Nutzen aller verfügbaren Alternativen. Hieraus resultiert ein Wertebereich zwischen null und eins, wobei sich die Summe aller Wahlwahrscheinlichkeiten zu eins aufaddiert. Das MNL-Modell ist allerdings nur unter der sog. IIA-Annahme (Independence of Irrelevant Alternatives) gültig, bei der von einem festen Substitutionsmuster ausgegangen wird (URBAN, 1993). Diese Annahme besagt, dass die Störterme e unabhängig von den anderen Alternativen im Choice-Set $C$ sind. Es wird also zum einen angenommen, dass sich das Verhältnis von zwei Alternativen unabhängig von den übrigen zur Wahl stehenden Alternativen nicht ändert. Allerdings erweist sich diese Annahme in vielen Fällen als unrealistisch, da sich die verfügbaren Alternativen sehr wohl auf die Wahlwahrscheinlichkeit auswirken können. Ferner wird innerhalb des MNL-Modells vorausgesetzt, dass der Parametervektor $\beta$ der Nutzenfunktion über alle Befragten identisch ist. Auch diese Annahme lässt sich in der Realität nur schwer aufrechterhalten, da die Präferenzen einzelner Alternativen und Einflussfaktoren zwischen den Befragten variieren können (TEMME, 2009). So können beispielsweise flexible Arbeitszeiten für freizeitorientierte Personen einen höheren Stellenwert einnehmen als für Personen, die ihrer Freizeit keine besondere Bedeutung beimessen. Das MNL-Modell kann solche individuellen Unterschiede im Responseverhalten nur eingeschränkt berücksichtigen. Während also beobachtbare Unterschiede, wie beispielsweise Präferenzen, adäquat berücksichtigt werden können, gelingt dies bei der unbeobachteten Heterogenität in Form von individuellen Unterschieden nicht (URBAN, 1993). Infolgedessen kann es beim MNL-Modell zu verzerrten Parameterschätzungen 
kommen, was wiederum zu nicht validen Schlussfolgerungen führt. Ein alternatives Modell, in dem sowohl die Berücksichtigung unbeobachteter Heterogenität zwischen den Befragten als auch die Verletzung bzw. Aufhebung der IIA-Annahme zulässig ist, ist das Mixed-MultinomialLogit-Modell (TEMME, 2009). Das Ziel dieses Modells besteht darin, unter Aufrechterhaltung der Nutzenmaximierungsannahme die Wahlwahrscheinlichkeiten flexibler als im MNL-Modell zu modellieren, indem bestimmte Attribute der Befragten als entscheidungsträgerspezifische Parameter in der Nutzenfunktion berücksichtigt werden. URBAN (1993) zählt zu solchen entscheidungsträgerspezifischen Attributen beispielsweise soziodemographische Variablen, Einstellungen oder Werthaltungen und bezeichnet diese als X-Variablen. Demgegenüber werden alternativenspezifische Attribute, wie beispielsweise das Arbeitgeberimage im Falle des Produktes Stellenangebot, Z-Variablen genannt. Mithilfe von Mixed-Logit-Modelle können sowohl entscheidungsträgerspezifische als auch alternativenspezifische Attribute in einem Modell geschätzt werden Es ergibt sich die folgende Wahlwahrscheinlichkeit (URBAN, 1993):

$$
P_{i j}=\frac{\exp \left(\gamma Z_{i j}+\beta X_{i}\right)}{\sum_{j=1}^{J} \exp \left(\gamma Z_{i j}+\beta X_{i}\right)}
$$

Das Mixed-Logit-Modell kam hier zum Einsatz, da sowohl entscheidungsträgerspezifische als auch alternativenspezifische Attribute gemeinsam modelliert werden sollen. Die Schätzung des Choice-Modells erfolgte in dieser Arbeit mit dem Softwareprogramm STATA 10.0. Die Entscheidung, die None-Option bei der weiterführenden Analyse nicht zu berücksichtigen, stützte sich neben den oben dargestellten methodischen Aspekten auf einem statistischen Modellvergleich, bei dem das Modell einmal mit dem vollständigen Datensatz und einmal mit dem Datensatz ohne die None-Option geschätzt wurde. Zur Modellbewertung wurde das sog. AkaikeInformationskriterium (AIC) herangezogen, dessen ausführliche Darstellung sich beispielsweise bei KUHA (2004) findet. Allgemein gilt, dass der Modellfit umso besser ist, je geringer der AIC-Wert ist. Wie der Vergleich zeigt, weist dieses Kriterium das Modell ohne die None-Option deutlich als überlegenes Modell aus.

\begin{tabular}{|l|l|l|}
\hline Kriterium & Modell mit None-Option & Modell ohne None-Option \\
\hline N & 6036 & 4104 \\
\hline AIC & 3096,14 & 1943,81 \\
\hline
\end{tabular}

Tab. 6-7: Modellvergleich anhand des AIC (Eigene Berechnungen, 2012)

Nach der Eliminierung der None-Option aus dem Datensatz waren für die anschließend durchgeführte Analyse insgesamt 4104 Wahlentscheidungen verfügbar. Die fünf Arbeitsplatzmerkmale wurden dabei als Dummy-Variablen unter Auslassung einer Referenzkategorie in das Discrete-Choice-Modell eingeführt. 


\begin{tabular}{|c|c|c|c|}
\hline Variable & b-Koeffizient & z-Wert & $\operatorname{LR}-\mathrm{Chi}^{2}(\mathrm{df}=1)$ \\
\hline \multicolumn{4}{|c|}{ Grundmodell } \\
\hline $\begin{array}{l}\text { Entwicklungs- und Aufstiegs- } \\
\text { chancen (eac) }\end{array}$ & 1,61 & $11,84 * * *$ & \\
\hline $\begin{array}{l}\text { Vereinbarkeit Beruf und } \\
\text { Familie/Privatleben (vbf) }\end{array}$ & 0,89 & $11,59 * * *$ & \\
\hline Bezahlung (bez) & $-0,27$ & $-2,48^{*}$ & \\
\hline Arbeitsplatzsicherheit (aps) & 1,58 & $19,19 * * *$ & \\
\hline $\begin{array}{l}\text { Gütesiegel „audit } \\
\text { berufundfamilie“ (gts2) }\end{array}$ & 0,70 & $5,60 * * *$ & \\
\hline Gütesiegel „Top Job“ (gts3) & 0,68 & $5,62 * * *$ & \\
\hline \multicolumn{4}{|c|}{ Interaktionseffekte } \\
\hline eac*Karriereorientierung & 0,22 & $2,36^{*}$ & $5,57^{*}$ \\
\hline vbf*Freizeitorientierung & 0,35 & $6,56^{* * *}$ & $45,59 * * *$ \\
\hline bez*Karriereorientierung & 0,07 & 0,96 & 0,93 \\
\hline gts $2 *$ Freizeitorientierung & 0,05 & 0,93 & 0,87 \\
\hline gts $3 *$ Karriereorientierung & $-0,03$ & $-0,60$ & 0,36 \\
\hline gts $2 *$ Bekanntheit & $-0,50$ & $-2,47 *$ & $6,16^{*}$ \\
\hline gts3*Bekanntheit & 0,05 & 0,25 & 0,06 \\
\hline gts $2 *$ Relevanz & 0,28 & $2,67 * *$ & $7,16^{* *}$ \\
\hline gts3*Relevanz & 0,16 & 1,52 & 2,31 \\
\hline \multicolumn{4}{|c|}{ Positionseffekte } \\
\hline $\begin{array}{l}\text { positionsspezifische Kontante } \\
2 \text { (psc2) }\end{array}$ & 0,10 & 1,17 & \\
\hline $\begin{array}{l}\text { positionsspezifische Kontante } \\
3 \text { (psc3) }\end{array}$ & 0,06 & 0,62 & \\
\hline $\begin{array}{l}\mathrm{N}=4104, \text { LR-Statistik }=1095,99 * \\
\text { Signifikanzniveaus: } * \mathrm{p}<.05, * * 1\end{array}$ & LL-Wert $=-954$ & & \\
\hline
\end{tabular}

Tab. 6-8: Schätzergebnisse des Discrete-Choice-Modells (Eigene Berechnungen, 2012) 
Zur Beurteilung der Modellgüte wird zum einen die Pseudo- $\mathrm{R}^{2}$-Statistik, genauer das Pseudo$\mathrm{R}^{2}$ von McFadden, herangezogen (KOHLER und KREUTER, 2008). Entsprechende Werte über 0,2 werden als akzeptabel und Werte über 0,4 als gut beurteilt. Ein Wert nahe eins bedeutet, dass die im Modell berücksichtigten unabhängigen Variablen einen bedeutenden Einfluss haben, während ein Wert nahe null auf eine geringe Bedeutung hinweist. Bei dem hier geschätzten Modell resultiert ein $\mathrm{R}_{\mathrm{MF}}^{2}$-Wert von 0,365. Die Modellanpassung wird demzufolge als sehr zufriedenstellend betrachtet.

Ein weiteres Beurteilungsmaß für die Güte des Gesamtmodells bietet der Likelihood-RatioTest (LR), bei dem zwei Modelle geschätzt und die daraus resultierenden LogLikelihood-Werte (LL) substrahiert werden. Dabei wird die Likelihood des vollständig geschätzten Modells $\left(\mathrm{LL}_{\mathrm{v}}\right)$ mit einem Modell verglichen, das nur die Konstante enthält $\left(\mathrm{LL}_{0}\right)$.

$$
\mathrm{LR}=\mathrm{LL}_{0}-\mathrm{LL}_{\mathrm{v}}
$$

Je größer die Differenz für J Freiheitsgrade ausfällt, desto höher wiegt die Bedeutung des geschätzten Modells bzw. der im vollständigen Modell berücksichtigten unabhängigen Variablen (BACKHAUS et al., 2011). Der LR-Wert folgt einer Chi $^{2}$-Verteilung und prüft die Hypothese, dass alle Koeffizienten bis auf die Konstante null sind. Der hier durchgeführte Likelihood-RatioTest ergibt ein hoch signifikantes Ergebnis $\left(\mathrm{LR}-\chi_{(17, \mathrm{~N}=4104)}^{2}=1095,99, \mathrm{p}<.001\right)$, aus dem folglich hervorgeht, dass mindestens einer der b-Koeffizienten in der Grundgesamtheit nicht null ist.

Die Schätzkoeffizienten des MNL-Modells sind gemäß Gleichung (8) nicht linear mit der endogenen Variablen verknüpft, was zugleich die Interpretation der Ergebnisse komplizierter macht. Infolgedessen erfolgte die Interpretation der Koeffizienten anhand der entsprechenden z-Werte, bei denen zum einen ihre Vorzeichen und zum anderen ihre relativen Größe herangezogen werden. So lassen sich aus den Vorzeichen Aussagen zur Wirkungsrichtung ableiten, während die standardisierten z-Werte einen direkten Vergleich unter den Koeffizienten innerhalb der Modellschätzung ermöglichen. Ein positives Vorzeichen eines Koeffizienten bedeutet demnach, dass die Wahrscheinlichkeit, ein bestimmtes Stellenangebot zu wählen, mit der jeweiligen Ausprägung steigt, während ein negatives Vorzeichen auf eine verringerte Wahrscheinlichkeit hinweist. Über die z-Werte lässt sich zudem das relative Ausmaß dieser Veränderung bestimmen, die umso stärker ist, je höher der Betrag des geschätzten z-Wertes ist. Die Schätzkoeffizienten dürfen indes aufgrund unterschiedlicher Standardfehler nicht miteinander verglichen werden.

Analog zur Likelihood des berechneten Modells lässt sich mithilfe des LR-Tests feststellen, welchen Einfluss eine oder mehrere unabhängige Variablen auf die Erklärungskraft des Modells haben, indem das Modell jeweils mit und ohne die relevante Variable geschätzt wird. So wurde für alle Interaktionsvariablen einzeln überprüft, ob sich die Modellgüte verbessert, wenn sie 
als zusätzliche Variablen in das Modell aufgenommen werden. Mit dem LR-Test lassen sich allerdings nur untereinander geschichtete Modelle vergleichen, sodass im vollständigen Modell alle Variablen des reduzierten Modells enthalten sein müssen. Die Teststatistik errechnet sich anhand der mit -2 multiplizierten Differenz zwischen der logarithmierten Likelihood eines Modells mit und ohne die zu untersuchende Variable (KOHLER und KREUTER, 2008). Auch diese Prüfgröße ist $\mathrm{Chi}^{2}$-verteilt, wobei die Anzahl der Freiheitsgrade der Differenz der Parameteranzahl der beiden Modelle, also in diesem Fall eins entspricht.

Aus den Ergebnissen der Modellschätzung in Tabelle 6-8 geht insgesamt hervor, dass zehn der dreizehn unabhängigen Variablen signifikant bis hoch signifikant ausfallen und somit den Nutzen des Stellenangebotes deutlich beeinflussen. Betrachtet man die Ergebnisse im Einzelnen, so zeigt sich zunächst, dass alle Koeffizienten der fünf Arbeitsplatzmerkmale im Grundmodell einen signifikanten Einfluss auf die Wahl des Stellenangebotes ausüben. Während die positiven Vorzeichen der geschätzten Parameter den Erwartungen entsprechen, fällt der Koeffizient der leistungsorientierten Vergütung wider Erwarten negativ aus $(z-W e r t=-2,48)$. Das bedeutet, dass eine solche Auslobung zu einem geringeren Nutzen und dementsprechend zu einer geringeren Wahlwahrscheinlichkeit eines Stellenangebotes führt. Möglicherweise ist dieses Ergebnis damit zu erklären, dass es sich bei den Befragten um potenzielle Bewerber mit keiner bzw. einer sehr geringen Berufserfahrung handelt. Die Befragten zeichneten sich also eher dadurch aus, dass sie hinsichtlich ihres Leistungsvermögens im Arbeitsleben nur wenige oder keine Erfahrungen hatten und eine just auf dieser Grundlage basierende Vergütung bei ihnen deshalb eher mit Unsicherheit einherging. Hypothesenkonform zeigt sich indes, dass das Vorzeichen des Interaktionsterms aus leistungsorientierter Vergütung und Karriereorientierung positiv ausfällt, wobei der dazugehörige Koeffizient nicht signifikant ist. Die negative Wirkung einer leistungsorientierten Bezahlung wird somit bei den eher karriereorientierten Befragten entkräftet.

Im Hinblick auf die relative Größe der Koeffizienten im Grundmodell zeigt sich, dass die Arbeitsplatzsicherheit hier die größte Bedeutung einnimmt $(z-W e r t=19,19)$. Analog zur leistungsorientierten Bezahlung ist auch hier zu vermuten, dass potenzielle Bewerber bei ihrem Berufseinstieg befristete und somit eher unsichere Arbeitsverhältnisse meiden wollen und deshalb den Nutzen unbefristeter Arbeitsverträge als besonders hoch einschätzen. Die beiden Koeffizienten der Entwicklungs- und Aufstiegschancen $(z-W e r t=11,84)$ und der Vereinbarkeit von Beruf und Familie/Privatleben $(z-W e r t=11,59)$ fallen in etwa gleich groß aus und besagen somit, dass beide Attribute einen ähnlichen Nutzen und eine vergleichbare Wahlwahrscheinlichkeit aufweisen. Die Ähnlichkeit in der relativ hohen Gewichtung beider Attribute ist insofern interessant, als dass es sich bei den Befragten um eine vergleichsweise junge Zielgruppe handelte (Altersdurchschnitt $=24$ Jahre). Dieses Ergebnis lässt sich zugleich so deuten, dass potenzielle Bewerber unterschiedliche Erwartungen an ihren Arbeitgeber haben, die sich wiederum in ihren Berufsorientierungen widerspiegeln. Ihr differenzieller Einfluss zeigt sich deutlich darin, dass die beiden postulierten Interaktionseffekte aus den Entwicklungs- und Aufstiegschancen 
und der Karriereorientierung $(\mathrm{z}$-Wert $=2,36)$ sowie aus der Vereinbarkeit von Beruf und Familie/Privatleben und der Freizeitorientierung $(\mathrm{z}$-Wert $=6,56)$ signifikant bzw. sehr signifikant ausfallen und die Wahlwahrscheinlichkeit entsprechend erhöhen.

Eine wesentliche Forschungsfrage dieses Teilmodells besteht ferner darin, ob und inwieweit Gütesiegel den Nutzen und die Wahlwahrscheinlichkeit eines bestimmten Stellenangebots beeinflussen. Dabei wurde zuerst der Frage nachgegangen, ob die Befragten bereits im Vorfeld der Studie mit den beiden Gütesiegeln vertraut waren.

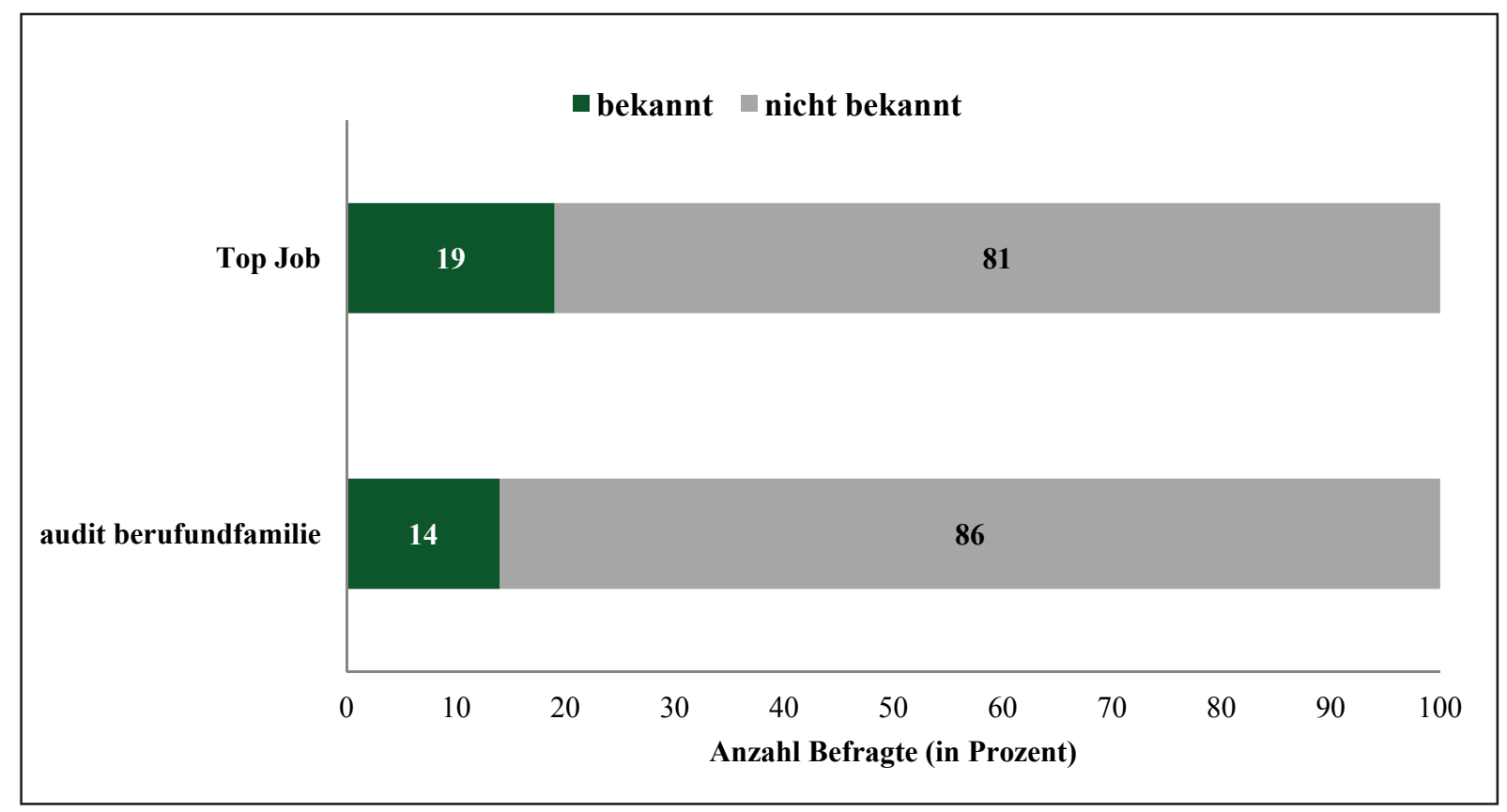

Abb. 6-1: Bekanntheitsgrad der beiden Gütesiegeln (Eigene Berechnungen, 2012)

Es zeigt sich, dass der Bekanntheitsgrad mit 14 Prozent für ,audit berufundfamilie“ bzw. 19 Prozent für „Top Job“ vergleichsweise gering ausfällt und somit nur ein kleiner Anteil der Befragten mit den beiden Gütesiegeln schon vorher konfrontiert wurde. Die nachfolgend durchgeführten statistischen Analysen stützen zunächst die Annahme, der zufolge Gütesiegel ein entscheidungsrelevantes Merkmal darstellen, da beide Koeffizienten sehr signifikant sind und somit die Wahlwahrscheinlichkeit des Stellenangebotes deutlich beeinflussen. Die Vorzeichen der geschätzten Parameter fallen erwartungsgemäß positiv und in etwa gleich hoch aus, d.h. das Vorhandensein eines der beiden Gütesiegel führt zu einem höheren Nutzen und folglich zu einer höheren Wahlwahrscheinlichkeit. Im Vergleich zu den übrigen positiven Parametern fallen sie in ihrer Höhe geringer aus, sodass ihr relativer Einfluss auch entsprechend schwächer sein sollte. Betrachtet man allerdings die beiden Interaktionseffekte aus dem Gütesiegel und seinem jeweiligen Bekanntheitsgrad, so ergibt sich ein differenzierteres Bild: Im Falle seiner Bekanntheit übt das Gütesiegel „Top Job“ keinen signifikanten Einfluss auf die Wahlwahrscheinlichkeit des Stellenangebotes aus, während der entsprechende Koeffizient des Gütesiegels ,audit berufundfamilie“ ein negatives Vorzeichen aufweist und demzufolge sogar zu einer 
geringeren Wahlwahrscheinlichkeit führt $(z-$ Wert $=-2,47)$. Der erstgenannte Interaktionseffekt kann möglicherweise dadurch erklärt werden, dass die im Gütesiegel transportierte Botschaft eher diffus erscheint und deshalb bereits anhand der übrigen, näher definierten Arbeitsplatzmerkmale ausreichend abgebildet ist, sodass das Gütesiegel keinen weiterführenden Informationsgewinn ermöglichen. Währenddessen lässt sich das negative Koeffizientenvorzeichen des Gütesiegels „audit berufundfamilie“ so interpretieren, dass die Kenntnis des Gütesiegels, beispielsweise von anderen Stellenangeboten oder aus den Medien, in einem inflationären Eindruck hinsichtlich seiner Verwendung mündet. Eine weitere Interpretationsmöglichkeit wäre auch darin zu sehen, dass das Gütesiegel insbesondere auf die Vereinbarkeit mit der Familie und damit einhergehende Maßnahmen fokussiert. Möglicherweise ist dieser Lebensbereich für die vergleichsweise jungen Befragten bislang noch nicht sonderlich relevant, was wiederum in einem verringerten Nutzen des Stellenangebotes resultiert. Hinsichtlich ihrer differenziellen Wirkung ergeben sich bei beiden Gütesiegeln nicht signifikante Interaktionseffekte mit den individuellen Berufsorientierungen. Die Annahme, dass das Gütesiegel „Top Job“ bzw. „,audit berufundfamilie“ bei karriere- bzw. freizeitorientierten Befragten mit einem größeren Nutzen und einer höheren Wahlwahrscheinlichkeit einhergeht, ist somit in beiden Fällen zurückzuweisen. Eine mögliche Erklärung für dieses Ergebnis könnte darin liegen, dass karriere- bzw. freizeitorientierte Befragte den Nutzen des Stellenangebotes ausreichend anhand der vorangegangenen Arbeitsplatzmerkmale einschätzen können und in den beiden Gütesiegeln deshalb keinen darüber hinaus gehenden Nutzenaspekt sehen.

Im nächsten Schritt wurde die Hypothese überprüft, ob und inwieweit die Nutzenbewertung des Gütesiegel von seiner generellen Bedeutung für die Befragten abhängt.

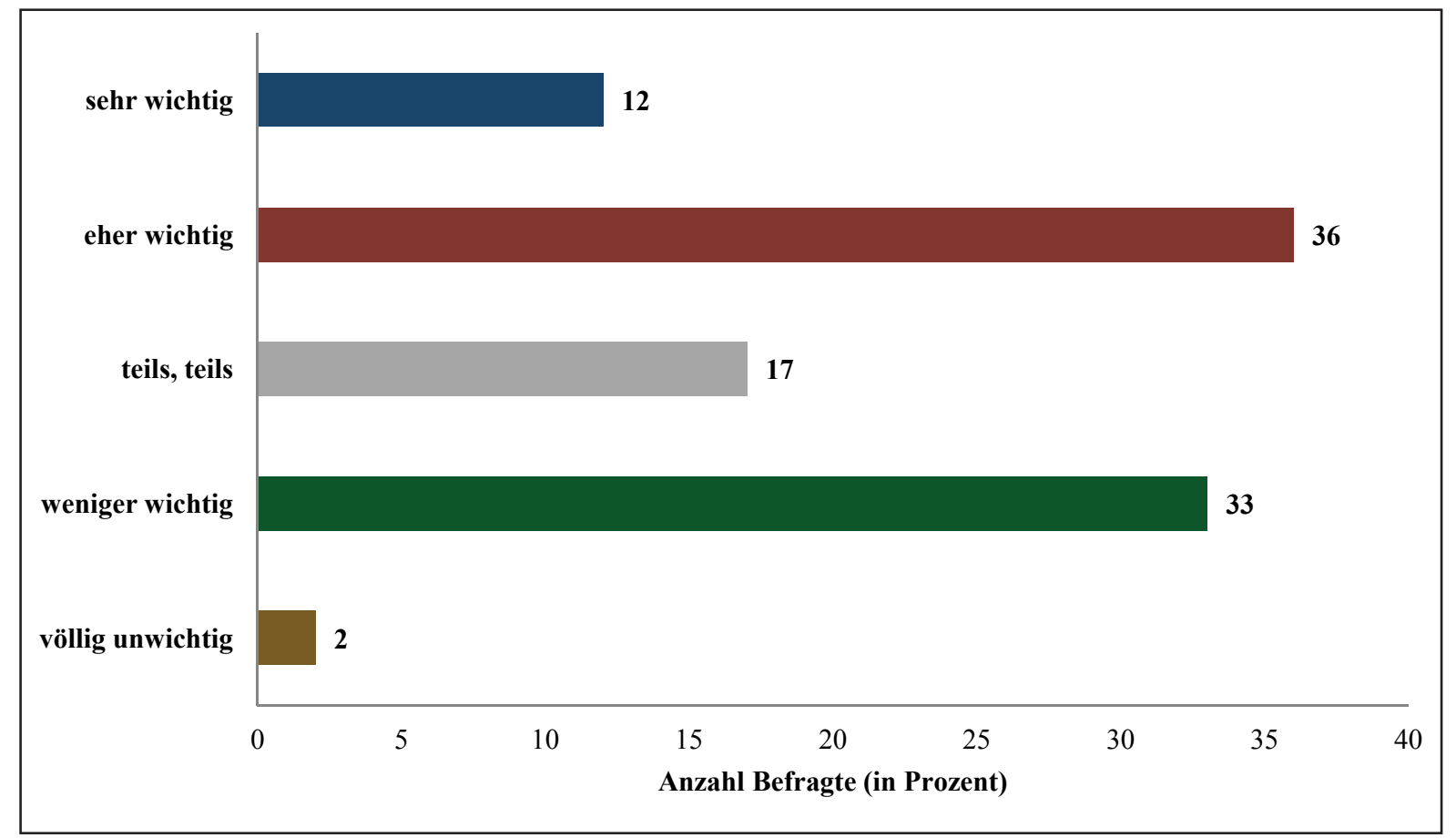

Abb. 6-2: Eingeschätzte Relevanz der Gütesiegel bei der Arbeitgeberwahl (Eigene Berechnungen, 2012) 
Hierbei gab knapp die Hälfte (= 48 Prozent) der Befragten an, einem Gütesiegel bei ihrer Arbeitgeberwahl generell eine hohe Bedeutung beizumessen. Dieses Ergebnis zeigt, dass Gütesiegel grundsätzlich ein entscheidungsrelevantes Merkmal darstellen könnten. Die statistischen Ergebnisse ergeben ein differenzierteres Bild: Während das Gütesiegel „,audit berufundfamilie“ bei Befragten, die einem Gütesiegel grundsätzlich einen höheren Stellenwert einräumen, zu einem signifikant größeren Nutzen und einer höheren Wahlwahrscheinlichkeit führt (z-Wert = 2,67), fällt der Parameter des zweiten Interaktionseffektes nicht signifikant aus. Die Annahme, dass mit steigender Relevanz des Gütesiegels auch sein Einfluss auf die Wahl eines Stellenangebotes vergrößert wird, muss für das Gütesiegel „Top Job“ daher zurückgewiesen werden. Im anschließend durchgeführten LR-Test wurde für alle Interaktionseffekte einzeln die Wahrscheinlichkeit berechnet, einen bestimmten LR-Chi²-Wert in einer Stichprobe zu erhalten, wenn der jeweiligen Koeffizienten in der Grundgesamtheit null ist. Die Ergebnisse zeigen, dass die Irrtumswahrscheinlichkeit für alle signifikanten Parameter sehr klein ist, sodass die entsprechenden Koeffizienten in der Grundgesamtheit nicht null sind. Schließlich geht aus dem hier geschätzten Discrete-Choice-Modell hervor, dass die beiden positionsspezifischen Konstanten nicht signifikant ausfallen. Das bedeutet, dass die Anordnung der Alternativen im Choice-Set keinen signifikanten Einfluss auf die Wahlwahrscheinlichkeit hatte und die Randomisierung somit erfolgreich war.

\subsection{Analyse des dritten Teilmodells: Erklärung der Bewerbungsintention}

Basierend auf den im Abschnitt 4.4 aufgestellten Hypothesen wurde ein Kausalmodell entwickelt, mit dem sich der Einfluss der einzelnen Determinanten auf die Bewerbungsintention bestimmen lässt. Zur Modellierung des Kausalmodells und dessen Messung wurde die statistische Software SmartPLS 2.0 (RINGLE et al., 2005) eingesetzt.

\subsubsection{Beurteilung des Messmodells}

Bei der Analyse des Messmodells gilt es, die Indikatorreliabilitäten sowie ihre konvergente und diskriminante Vailidität zu beurteilen (WEIBER und MÜHLhaUS, 2010). So wird anhand der Indikatorreliabilität festgestellt, wie hoch der Varianzanteil eines Indikators ist, der durch das Konstrukt erklärt wird. Der Varianzanteil lässt sich dabei anhand der jeweiligen Ladung des einem Konstrukt zugeordneten reflektiven Indikatoren ablesen. Ein solches Item gilt demzufolge dann als reliabel, wenn mindestens 50 Prozent seiner Varianz durch das entsprechende Konstrukt erklärt werden, was wiederum bei einer Ladung von mehr als 0,7 der Fall ist. Neben der Bestimmung der Indikatorreliabilität stellt die konvergente Validität der Konstrukte ein wesentliches Gütekriterium des Messmodells dar. Diese kann zum einen an- 
hand der internen Konsistenz und zum anderen anhand der durchschnittlich erfassten Varianz ermittelt werden. Die interne Konsistenz gibt an, wie gut ein Konstrukt mittels der ihm zugeordneten Items gemessen wird. Neben Cronbachs Alpha lässt sich die interne Konsistenz anhand der sog. Faktorreliabilität (FR) bestimmen, die sich aus der Gesamtsumme aller Indikatoren eines Konstruktes zusammensetzt und somit der Indikatorreliabilität auf der Konstruktebene entspricht (NITZL, 2010). Als Richtwert für die Faktoreliabilität wird ein Wert von größer als 0,6 angegeben. Daneben dient die durchschnittlich extrahierte Varianz als weiteres Gütekriterium der konvergenten Validität. Das entsprechende AVE-Maß beziffert anteilig die mittels der latenten Variablen erklärte und die auf ihren Messfehler zurückzuführende Varianz. Während bei Cronbachs Alpha ein Wert von 0,7 als ausreichend betrachtet wird, sollte das AVE-Maß nicht weniger als 0,5 betragen, sodass mindestens 50 Prozent der Varianz eines Konstrukts durch die ihm zugrunde liegenden Indikatoren erklärt werden sollten.

Demgegenüber lässt sich aus der diskriminanten Validität das Ausmaß ableiten, inwieweit sich die Indikatoren eines Konstrukts von denen eines anderen Konstrukts desselben Modells unterscheiden (HENSELER et al., 2009). So gilt diese gemäß dem sog. FornellLarcker-Kriterium dann als erfüllt, wenn die Wurzel der durchschnittlich extrahierten Varianz eines Konstrukts größer ist als jede Korrelation dieses Konstrukts mit allen übrigen Konstrukten im Modell (FORNELL und LARCKER, 1981). Ergänzende Hinweise zur Erfüllung der diskriminanten Validität können zudem aus den sog. Kreuzladungen zwischen den reflektiven Indikatoren und den Konstrukten abgeleitet werden: Die Ladung eines reflektiven Indikators sollte mit dem ihm theoretisch zugeordneten Konstrukt höher ausfallen als mit allen anderen im Modell enthaltenen Konstrukten (NITZL, 2010).

Die Güte des hier aufgestellten Messmodells erwies sich als durchweg zufriedenstellend (vgl. Tab. 6-9). So zeigen die verschiedenen Indikatoren zum einen sehr hohe Reliabilitäten und liegen mit Werten größer als 0,7 allesamt über dem erforderlichen Richtwert. Ebenso ist die interne Konsistenz des Messmodells aufgrund der entsprechenden Werte für Cronbachs Alpha als erfüllt zu betrachten. Dieses Gütekriterium wird auch anhand der Faktorreliabilitäten bestätigt, die im Bereich von 0,853 bis 0,947 liegen und somit den erforderlichen Grenzwert von 0,6 überschreiten. Ferner erreicht die durchschnittlich extrahierte Varianz Werte von 0,66 bis 0,898 und liegt damit ebenfalls oberhalb des Grenzwertes von 0,6. Aus der Gesamtbetrachtung dieser Maße folgt zugleich, dass die Konvergenzvalidität des Messmodells ausreichend gesichert ist. Schließlich wurde im Hinblick auf die Diskriminanzvalidität sichergestellt, dass die vorliegenden AVE-Werte größer als alle quadrierten Korrelationen mit anderen Faktoren und somit dem Fornell-Larcker-Kriterium genügen. Folglich liegen diesem Kausalmodell durchweg valide Konstruktmessungen zugrunde. 


\begin{tabular}{|c|c|c|c|c|}
\hline Konstrukt & Ladung & AVE & FR & $\alpha$ \\
\hline Arbeitgeberattraktivität (Item 1-4) & $\begin{array}{l}0,903 \\
0,887 \\
0,909 \\
0,918\end{array}$ & 0,818 & 0,947 & .93 \\
\hline Prestige (Item 1-3) & $\begin{array}{l}0,929 \\
0,878 \\
0,910\end{array}$ & 0,821 & 0,932 & .89 \\
\hline $\begin{array}{l}\text { Erfolgswahrscheinlichkeit der Bewerbung } \\
\text { (Item 1-2) }\end{array}$ & $\begin{array}{l}0,948 \\
0,948\end{array}$ & 0,898 & 0,946 & .89 \\
\hline $\begin{array}{l}\text { Person-Organisation-Passung } \\
\text { (Item 1-3) }\end{array}$ & $\begin{array}{l}0,941 \\
0,916 \\
0,908\end{array}$ & 0,850 & 0,945 & .91 \\
\hline Bewerbungsabsicht (1 Item) & - & - & - & - \\
\hline Alternativen auf dem Arbeitsmarkt (1 Item) & - & - & - & - \\
\hline Branchenruf (Item 1-3) & $\begin{array}{l}0,752 \\
0,832 \\
0,850\end{array}$ & 0,660 & 0,853 & .74 \\
\hline Branchenwissen (Item 1-4) & $\begin{array}{l}0,826 \\
0,822 \\
0,893 \\
0,737\end{array}$ & 0,674 & 0,892 & .84 \\
\hline Richtwerte & $\geq 0,7$ & $\geq 0,5$ & $\geq 0,6$ & $\geq \mathbf{0 , 7}$ \\
\hline
\end{tabular}

Tab. 6-9: Güte der Messmodelle (Eigene Berechnungen, 2012)

\subsubsection{Beurteilung des Strukturmodells}

Im Anschluss an die Schätzung des Messmodells wurde das Strukturmodell berechnet, mit dem die postulierten Wirkbeziehungen überprüft werden sollten.

Zur Beurteilung des Strukturmodells werden neben den Bestimmtheitsmaßen und Modellparametern auch Maße für seine Stabilität und Prognosefähigkeit herangezogen (HENSELER et al., 2009). Zum einen stellen die Bestimmtheitsmaße mit den dazugehörigen $\mathrm{R}^{2}$-Werten der endogenen Variablen ein wesentliches Gütekriterium dar. Analog zur traditionellen Regressionsanalyse, in der das Gütemaß den Anteil der erklärten Streuung an der Gesamtstreuung wiedergibt, setzt es sich innerhalb der PLS-Analyse aus der Höhe bzw. dem Anteil der erklärten Varianz der abhängigen latenten Variable zusammen und misst somit die Anpassungsgüte einer Regressionsfunktion an die empirisch gewonnenen manifesten Indikatoren (BOSSOW-THIES und PANTEN, 2009). Dementsprechend sollte eine endogene Variable, die anhand der berücksichtigten Einflussfaktoren erklärt werden soll, ein möglichst hohes Bestimmtheitsmaß aufweisen, 
das aufgrund seiner Normierung Werte zwischen null und eins annehmen kann (KRAFFT et al., 2005). So gibt CHIN (1998) folgende $\mathrm{R}^{2}$-Werte zur Beurteilung von PLS-Modellen an: 0,19, 0,33 und 0,67 gelten als schwach, moderat und substanziell.

Darüber hinaus sind die Pfadkoeffizienten hinsichtlich ihrer Richtung, Höhe und Signifikanz zu bewerten (WEIBER und MÜHLHAUS, 2010). Auch ihre Interpretation lässt sich analog zu den Regressionskoeffizienten in einer Regressionsgleichung durchführen, wobei die einzelnen Pfadkoeffizienten wie standardisierte $\beta$-Koeffizienten interpretiert werden. Ihr Signifikanzniveau wird dabei anhand der t-Werte überprüft, die sich ihrerseits mithilfe der sog. Bootstrapping-Prozedur berechnen lassen, indem $\mathrm{n}$ unterschiedliche Zufallsstichproben vom Umfang N jeweils zur Schätzung des PLS-Modells herangezogen werden. Anhand der so geschätzten Parameter lassen sich die aufgestellten Hypothesen folgendermaßen bestätigen oder zurückweisen: Während nicht signifikante oder mit einem entgegengesetzten Vorzeichen versehenen Pfadkoeffizienten zur Ablehnung einer aufgestellten Hypothese führen, wird sie anhand signifikanter und vorzeichenkonformer Pfade bestätigt.

Neben den Signifikanzen erweisen sich die unterschiedlichen Effektstärken als wesentliche Gütekriterien des PLS-Modells, da hieraus abzuleiten ist, ob eine unabhängige latente Variable einen substanziellen Einfluss auf eine abhängige latente Variable ausübt (GÖTZ und LIEHRGOBBERS, 2004). Als statistisches Maß hierfür dient die Effektgröße $\mathrm{f}^{2}$, die das geänderte Bestimmtheitsmaß einer endogenen Variablen angibt, wenn der jeweilige Einfluss einer bestimmten exogenen Variablen ausgeschlossen wird. Demzufolge lässt sich die Effektgröße mathematisch folgendermaßen ausdrücken (vgl. NITZL, 2010):

$$
f^{2}=\frac{R_{\text {eingeschlossen }}^{2}-R_{\text {ausgeschlossem }}^{2}}{1-R_{\text {eingeschlossen }}^{2}}
$$

Je höher diese Effektgröße ist, desto größer ist der Einfluss der zugrunde liegenden exogenen Variablen. CHIN (1998) zufolge geben die entsprechenden Werte größer gleich 0,02, 0,15 sowie 0,35 an, ob eine exogene latente Variable einen schwachen, moderaten oder substanziellen Einfluss auf die ihr im Kausalmodell zugeordnete latente endogene Variable ausübt.

Ein weiteres Kriterium zur Modellbeurteilung von reflektiv gemessenen endogenen Variablen stellt die Prognoserelevanz $Q^{2}$ dar, die anhand des sog. Stone-Geisser-Kriteriums quantifiziert wird (WEIBER und MÜHLHAUS, 2010). Ausschlaggebend hierfür ist das grundlegende Prinzip, dass PLS die Prognose der Rohdaten anstrebt und nicht wie im LISREL-Ansatz auf die Reproduktion der Kovarianzstrukturen fokussiert (BOSSOW-THIES und PANTEN, 2009). Berechnet wird das Stone-Geisser-Kriterium mithilfe der sog. Blindfolding-Prozedur, worin ein Teil der Rohdatenmatrix zunächst systematisch als fehlend angenommen wird und die so ermittelten 
Parameterschätzungen im darauffolgenden Schritt zur Vorhersage der als fehlend angenommenen Rohdaten eingesetzt werden (GöTZ und LIEHR-GOBBERS, 2004). Die Prognoserelevanz fungiert somit als Maß für die Rekonstruktionsgüte des zugrunde liegenden Modells und berechnet sich wie folgt (vgl. NITZL, 2010):

$$
Q^{2}=1-\frac{\sum_{D} E_{D}}{\sum_{D} O_{D}}
$$

Die Prognosefehler werden dabei mithilfe der verbleibenden Daten aus der Blindfolding-Prozedur mittels eines Vergleichs zu den tatsächlichen Werten bestimmt (KRAFFT et al., 2005). Eine hinreichende Prognosefähigkeit des Modells ist bei einem Wert von größer null gegeben, während Werte kleiner als null auf eine geringe Prognosegüte der überprüften Modellstruktur hindeuten (WEIBER und MÜHLHAUS, 2010). Ein Wert von null besagt indes, dass die empirisch erhobenen Daten nicht besser vorhergesagt werden als mittels einer Schätzung per Mittelwert. Mit der Gütebeurteilung des Kausalmodells auf seiner Strukturebene gilt der Validierungsprozess innerhalb der PLS-Analyse als abgeschlossen.

Für das Bestimmtheitsmaß der zentralen endogenen Variablen Bewerbungsabsicht resultiert ein Wert von 0,576. Demnach kann hier ein als substanziell einzustufendes Bestimmtheitsmaß ermittelt und die Bewerbungsabsicht mit dem aufgestellten Modell zufriedenstellend erklärt werden. Auch das zweite besonders relevante endogene Konstrukt, die wahrgenommenen Arbeitgeberattraktivität, weist mit einem $\mathrm{R}^{2}$-Wert von 0,648 einen substanziellen Erklärungsanteil auf. Demgegenüber können die übrigen Konstrukte des Strukturmodells erwartungsgemäß nur zu einem geringen Anteil erklärt werden. Zugleich lässt sich anhand des hieraus resultierenden Stone-Geisser-Kriteriums zeigen, dass der $\mathrm{Q}^{2}$-Wert oberhalb von null liegt und das aufgestellte Modell zur Erklärung der Bewerbungsabsicht somit eine Vorhersagerelevanz besitzt. Aus den im Strukturmodell resultierenden Pfadkoeffizienten geht zudem hervor, dass bis auf eine Hypothese alle postulierten direkten Wirkbeziehungen bestätigt werden können. Während die Pfadkoeffizienten aller übrigen angenommenen Wirkbeziehungen größtenteils sehr signifikant oder gar hoch signifikant ausfallen, ist der Pfadkoeffizient zwischen dem Branchenruf und dem organisationalen Prestige nicht signifikant. Die Hypothese 19, der zufolge der Branchenruf der Ernährungsindustrie direkt das organisationale Prestige eines mittelständischen Unternehmens aus dieser Branche beeinflusst, wird somit zurückgewiesen. Zur weiterführenden Einschätzung, ob eine exogene Variable einen bedeutenden Einfluss auf die endogenen Variablen hat, wurden für alle endogenen Variablen, die mehr als eine Beziehung zu einer latent exogenen Variablen aufweisen, die Effektgrößen $\mathrm{f}^{2}$ berechnet. 


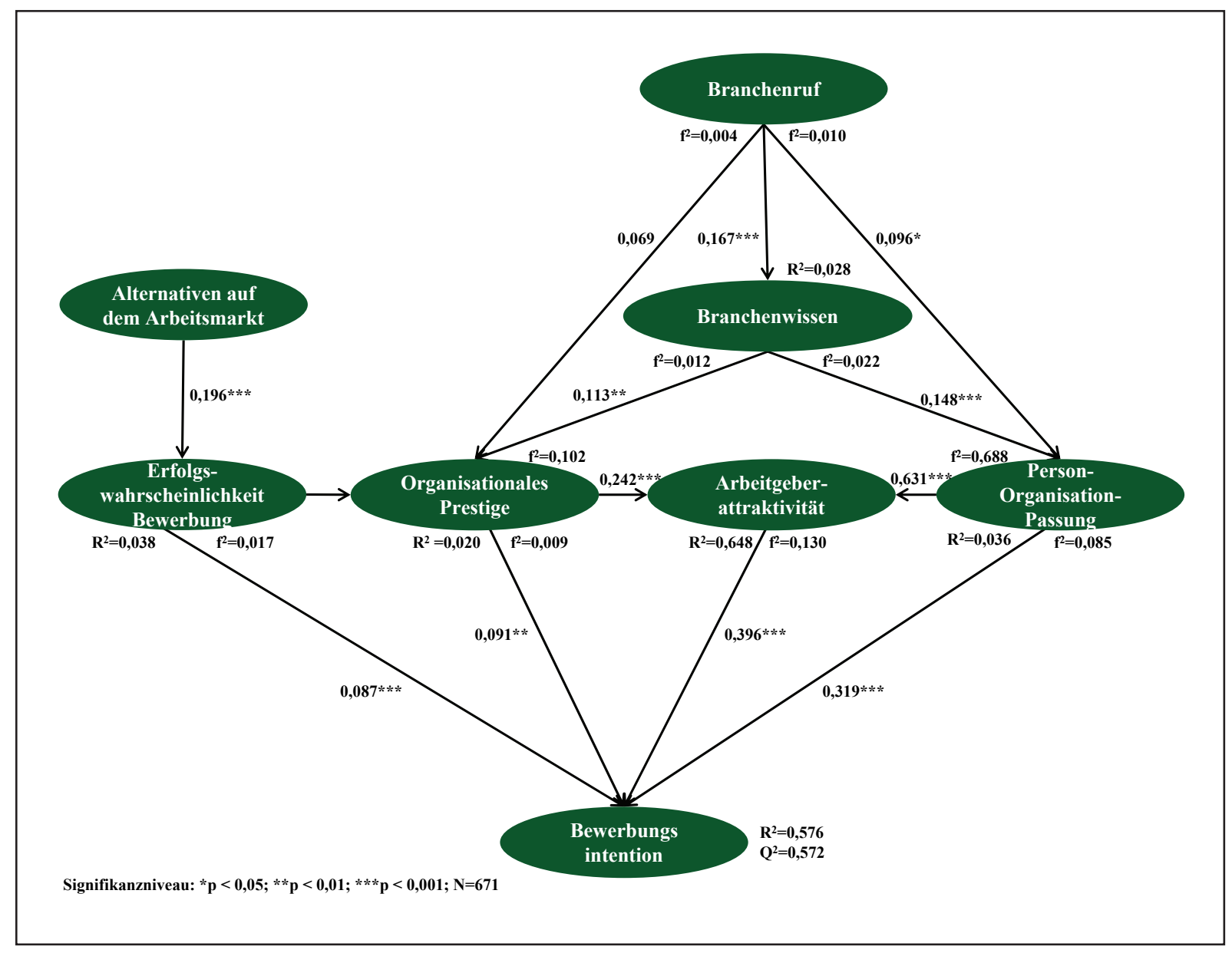

Abb. 6-3: Parameterschätzungen des Kausalmodells nach Bootstrapping mit 200 Stichproben

(Eigene Berechnungen, 2012)

Betrachtet man die wahrgenommene Arbeitgeberattraktivität, so lässt sich der Einfluss der exogenen Variablen Person-Organisation-Passung auf eine substanzielle Effektstärke von 0,688 beziffern, während der Erklärungsbeitrag des organisationalen Prestiges mit einem $\mathrm{f}^{2}$-Wert von 0,102 eher gering ausfällt. Bei der Bewerbungsabsicht ergeben sich insgesamt deutlich geringere oder gar gänzlich unbedeutende Effektstärken, unter denen die wahrgenommeine Arbeitgeberattraktivität über den größten Einfluss verfügt. Die wahrgenommene Arbeitgeberattraktivität hat somit die größte Bedeutung für die Bewerbungsabsicht, wenngleich es sich dabei um einen eher moderaten Effekt handelt.

Im Gegensatz zur Kovarianzstrukturanalyse, in der gleich mehrere globale Fitindizes zur Beurteilung der Gesamtgüte des betrachteten Modells bereitstehen, die ihrerseits anhand inferenzstatistischer Tests ermittelt werden, existiert innerhalb des PLS-Ansatzes aufgrund der wenig restriktiven Verteilungsannahmen ein solches Gütekriterium nicht (KRAFFT et al., 2005). Infolgedessen erfolgt die Modellbeurteilung kumulativ entlang aller oben aufgezeigten Gütekriterien. Ein Gesamtmodell erweist sich somit dann als zuverlässig, wenn sämtliche als relevant eingestuften Gütekriterien sowohl im Mess- als auch im Strukturmodell hinreichend erfüllt 
werden (HENSELER et al., 2009). Im Hinblick auf das hier überprüfte Kausalmodell wird aus einer solchen Gesamtbetrachtung deutlich, dass das Modell über eine akzeptable Eignung verfügt. Während bei den zugrunde liegenden reflektiven Messmodellen durchweg sehr zuverlässige und valide Kennwerte zu verzeichnen sind, ergeben sich im Strukturmodell vereinzelt auch nicht bedeutsame Wirkbeziehungen gepaart mit geringen Bestimmtheitsmaßen einiger endogener Konstrukte. Gleichwohl liegen für die beiden zentralen endogenen Variablen - wahrgenommene Arbeitgeberattraktivität sowie Bewerbungsabsicht - zufriedenstellende statistische Kennwerte vor, sodass das aufgestellte Kausalmodell in seiner Erklärungskraft insgesamt als gut zu bewerten ist.

\subsubsection{Modellierung mediierender Effekte}

Ergänzend zu den einfachen Wirkbeziehungen, in denen die exogene Variable direkt die endogene Variable beeinflusst, wurden vier mediierende Effekte modelliert. Eine mediierte Wirkbeziehung liegt dann vor, wenn der Effekt einer exogenen Variable auf die zu erklärende, endogene Variable teilweise oder vollständig durch eine dritte Variable vermittelt wird (BARON und KENNY, 1986). Werden mediierte Wirkbeziehungen in einem Kausalmodell unterstellt, jedoch nicht explizit überprüft, so verringert sich hierdurch die Gültigkeit der Modellschätzung (EGGERT et al., 2005). Ein Mediationseffekt wird anhand des in Abbildung 6-4 dargestellten Modells getestet.

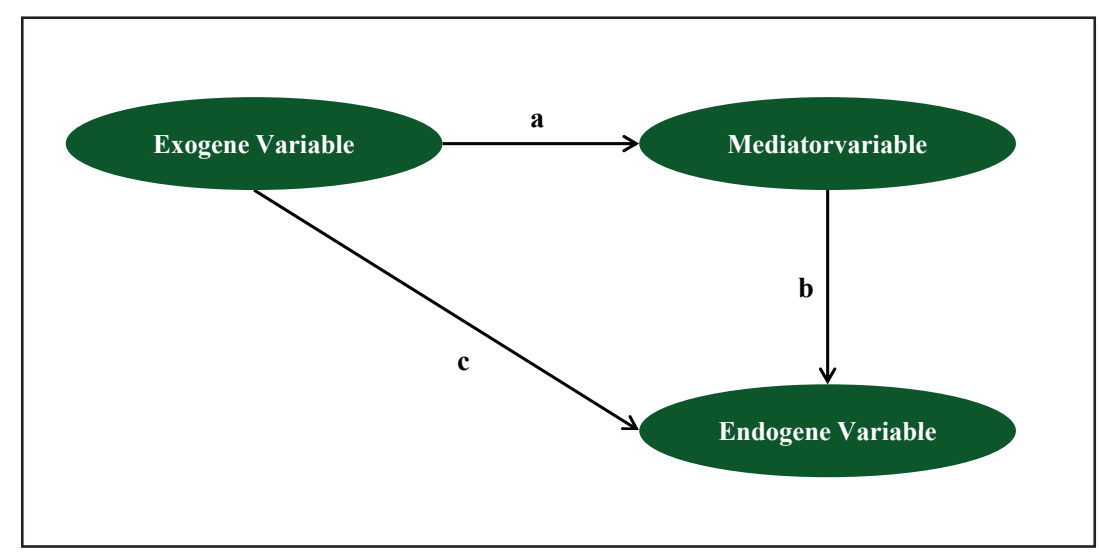

Abb. 6-4: Mediatorenmodell

(Eigene Darstellung, 2012, in Anlehnung an EGGERT et al., 2005)

Demzufolge liegt ein Mediationseffekt dann vor, wenn

1. graduelle Veränderungen der exogenen Variable zu signifikanten Effekten auf die mediierende Variable führen (Pfad a), 
2. Veränderungen der Mediatorvariable zu signifikanten Effekten auf die endogene Variable führen (Pfad b)

3. und der Pfad c signifikant kleiner ist, d.h. zwischen null und dem Pfad c' in einem Alternativmodell ohne Mediatorvariable liegt. Wenn der Pfad c sich nicht signifikant von null unterscheidet, dann liegt eine vollständige Mediation vor, andernfalls handelt es sich um eine partielle Mediation.

Um zu testen, ob ein Mediationseffekt vorliegt, kann der von SOBEL (1982) konzipierte zTest herangezogen werden. Dieser prüft die Signifikanz des indirekten Effektes der exogenen Variablen über den Mediator auf die endogene Variable. Die Testgröße z wird unter Berücksichtigung der Pfadkoeffizienten a und b sowie der entsprechenden Standardfehler von $a$ und $b$ gemäß der folgenden Formel ermittelt:

$$
z=\frac{a * b}{\sqrt{b^{2} * s_{a}^{2}+a^{2} * s_{b}^{2}}}
$$

Anschließend gilt es, das Ausmaß des mediierenden Effekts festzustellen. Als Kenngröße fungiert hierbei der VAF-Wert, bei dem die indirekte Wirkung der exogenen Variablen auf die endogene Variable ins Verhältnis zum Gesamteffekt gesetzt wird (vgl. EGGERT et al., 2005):

$$
V A F=\frac{a * b}{a * b+c}
$$

Demgemäß bedeutet beispielsweise ein VAF-Wert von 0,5, dass 50 Prozent des Einflusses, den eine exogene Variable auf die endogene Variable bewirkt, durch die Mediatorvariable verursacht wurden. Im Rahmen des aufgestellten Kausalmodells wurden folgende mediierende Effekte analysiert: Zum einen wurde angenommen, dass die wahrgenommene Arbeitgeberattraktivität sowohl den Einfluss der Person-Organisation-Passung als auch des organisationalen Prestiges auf die Bewerbungsabsicht vermittelt. Eine Steigerung der Bewerbungsabsicht über die Person-Organisation-Passung bzw. das organisationale Prestige wäre demnach nur dann möglich, wenn durch den Einfluss der beiden zuletzt genannten Konstrukte auch die Arbeitgeberattraktivität positiver ausfiele. Hierbei wird zugleich nicht angenommen, dass die PersonOrganisation-Passung bzw. das organisationale Prestige die Arbeitgeberattraktivität bewirkt, sondern vielmehr dass ein Effekt dieser beiden Konstrukte auf die Bewertung der Arbeitgeberattraktivität vorliegt. Anders ausgedrückt, führen die beiden Konstrukte nicht zur Arbeitgeberattraktivität, gleichwohl beeinflusst jedes Konstrukt für sich die individuelle Wahrnehmung eines Arbeitgebers durch einen potenziellen Bewerber. 
Analog dazu wurde überprüft, ob und inwieweit das Branchenwissen den Zusammenhang zwischen dem Branchenruf und der Person-Organisation-Passung bzw. dem organisationalen Prestige vermittelt. Auch hierbei wurde nicht davon ausgegangen, dass der Branchenruf zum Wissenserwerb führt, sondern dass ein Effekt des Branchenrufs auf die Aktivierung und Beurteilung des eigenen Branchenwissens vorliegt.

\begin{tabular}{|l|l|l|l|}
\hline Mediatorvariable & Mediierter Zusammenhang & z-Statisitk & VAF-Wert \\
\hline Arbeitgeberattraktivität & PO-Fit $\longrightarrow$ Bewerbungsabsicht & $7,802 * * *$ & $43,9 \%$ \\
\hline Arbeitgeberattraktivität & Prestige $\longrightarrow$ Bewerbungsabsicht & $5,408 * * *$ & $51,3 \%$ \\
\hline Branchenwissen & Branchenruf $\longrightarrow$ PO-Fit & $2,964 * *$ & $20,4 \%$ \\
\hline Branchenwissen & Branchenruf $\longrightarrow$ Organisationales Prestige & $2,297 *$ & $100 \%$ \\
\hline Signifikanzniveaus: ${ }^{*} \mathrm{p}<.05, * * \mathrm{p}<.01,{ }^{* * *} \mathrm{p}<.001$ & & \\
\hline
\end{tabular}

Tab. 6-10: Analyseergebnisse der mediierenden Effekte (Eigene Berechnungen, 2012)

Tabelle 6-10 verdeutlicht, dass ein hoch signifikanter Effekt der Person-Organisation-Passung auf die wahrgenommene Arbeitgeberattraktivität vorliegt $(0,631, \mathrm{p}<0,001)$. Zudem beeinflusst die wahrgenommene Arbeitgeberattraktivität die Bewerbungsabsicht $(0,396, \mathrm{p}<0,001)$. Da jedoch auch der direkte Effekt der Person-Organisation-Passung auf die Bewerbungsabsicht hoch signifikant ausfällt $(0,319, \mathrm{p}<0,001)$, geht mit der wahrgenommenen Arbeitgeberattraktivität nur eine partielle Mediation einher. Einen Beleg für diesen Mediationseffekt liefert der aus der z-Statistik resultierende Wert von 7,802. Dieses Ergebnis ist so zu interpretieren, dass die Person-Organisation-Passung sich sowohl direkt als auch indirekt über die wahrgenommene Arbeitgeberattraktivität auf die Bewerbungsabsicht auswirkt. Das Ausmaß der indirekten Wirkung der Person-Organisation-Passung auf die Bewerbungsabsicht veranschaulicht der VAF-Wert von 43,9 Prozent. Das bedeutet, dass mehr als 40 Prozent der Gesamtwirkung der Person-Organisation-Passung auf die Bewerbungsabsicht über die wahrgenommene Arbeitgeberattraktivität vermittelt werden.

Ferner wurde anhand der zweiten Mediationshypothese überprüft, ob und inwieweit die wahrgenommene Arbeitgeberattraktivität den Einfluss des organisationalen Prestiges auf die Bewerbungsabsicht kanalisiert. Da alle drei Koeffizienten signifikant ausfallen, ruft die wahrgenommene Arbeitgeberattraktivität auch hier nur einen partiellen Mediationseffekt hervor, dessen z-Statistik sich auf einen Wert von 5,408 beläuft. Aus dem dazugehörigen VAF-Wert von 51,3 Prozent folgt zugleich, dass mehr als die Hälfte des Gesamteinflusses des organisationalen Prestiges auf die Bewerbungsabsicht auf die wahrgenommene Arbeitgeberattraktivität zurückzuführen ist.

Darüber hinaus wurde der angenommene Mediationseffekt des Branchenrufs auf den Modellzusammenhang zwischen dem Branchenwissen und der Person-Organisation-Passung überprüft. 
Hierbei zeigen sich drei signifikante Effekte: Der Branchenruf beeinflusst das Branchenwissen $(0,167, \mathrm{p}<0,001)$, das Branchenwissen wirkt sich hoch signifikant auf die Person-Organisation-Passung aus $(0,148, \mathrm{p}<0,001)$ und auch der direkte Einfluss des Branchenrufs auf die Person-Organisation-Passung fällt signifikant aus $(0,096, \mathrm{p}<0,05)$. Diese Konstellation deutet ebenfalls auf eine partielle Mediation hin, die durch die z-Statistik von 2,964 bestätigt wird. Das Ausmaß der indirekten Wirkung des Branchenrufs über das Branchenwissen auf die Person-Organisation-Passung verdeutlicht der resultierende VAF-Wert von 20,4 Prozent.

Schließlich wurde die vierte Mediatorhypothese überprüft, der zufolge das Branchenwissen den Einfluss des Branchenrufs auf das organisationale Prestige vermittelt. Während die beiden indirekten Pfadkoeffizienten hoch bzw. sehr signifikant ausfallen $(0,167, p<0,001$ und 0,113, $\mathrm{p}<0,01$ ), ist der direkte Pfadkoeffizient zwischen Branchenruf und organisationalem Prestige nicht signifikant. Aus der z-Statistik resultiert ein signifikanter Wert von 2,297. Infolgedessen liegt hier ein vollständig mediierter Effekt des Branchenwissens vor. Dieses Ergebnis ist allerdings insoweit einzuschränken, als dass aufgrund eines nicht signifikanten Pfadkoeffizienten die entsprechende Alternativhypothese nicht gleich zurückzuweisen, sondern erst unter der Bedingung einer ausreichenden Teststärke zu überprüfen ist. Die Teststärke umfasst dabei die Wahrscheinlichkeit, mit der ein Signifikanztest zugunsten der Alternativhypothese entscheidet, wenn diese gültig ist (BORTZ und SCHUSTER, 2010). Es handelt sich also um die Wahrscheinlichkeit, mit der ein tatsächlich von null verschiedener Pfadkoeffizient durch den eingesetzten statistischen Test aufzudecken ist. COHEN (1988) empfiehlt ein $\alpha / \beta$-Fehler-Verhältnis von $1 / 4$ mit einem $\alpha$-Fehler von 5 Prozent und einem $\beta$-Fehler von 20 Prozent, woraus eine mindestens erforderliche Teststärke $(=1-\beta)$ von 80 Prozent resultiert. Falls das Risiko, eine richtige $\mathrm{HO}$ zu verwerfen, als genauso gravierend betrachtet wird, wie das Risiko der Zurückweisung einer gültigen H1, dann gilt eine höhere Teststärke von 95 Prozent (BORTZ und SCHUSTER, 2010). Die $\alpha$-Fehlerwahrscheinlichkeit, Teststärke, Effektgröße und Stichprobenumfang sind zugleich funktional miteinander verknüpft, sodass beim Vorliegen von drei Größen die vierte eindeutig bestimmt werden kann (BORG und STAUFENBIEL, 2007). Aus den bei COHEN (1988) aufgelisteten Werten für die Teststärke von t-Tests lässt sich unter der Annahme einer kleinen Effektgröße von 0,2, einem Signifikanzniveau von 0,05 und dem Stichprobenumfang von N= 671 eine Teststärke von 95 Prozent ableiten. Dies belegt die getroffene Schlussfolgerung, dass das Branchenwissen einen vollständig mediierenden Effekt auf die Wirkbeziehung zwischen dem Branchenruf und dem organisationalen Prestige ausübt.

\subsection{Zusammenfassende Darstellung der Hypothesenprüfung}

Um eine übersichtliche Struktur der oben dargestellten Ergebnisse zu erhalten, werden alle Hypothesen und die dazugehörigen empirischen Befunde tabellarisch aufgeführt. Dabei wird 
für jede Hypothese gesondert angegeben, ob diese bestätigt oder zurückgewiesen wurde. Insgesamt ist festzustellen, dass die meisten Hypothesen ihrer empirischen Überprüfung standhalten konnten. Eine ausführliche Diskussion der Ergebnisse wird im nachstehenden Kapitel 7 erfolgen.

\begin{tabular}{|c|c|c|}
\hline Hypothese & Inhaltliche Fragestellung & Ergebnis \\
\hline \multicolumn{3}{|c|}{ Teilmodell 1} \\
\hline $\mathrm{H} 1$ & $\begin{array}{l}\text { Es wird angenommen, dass der Bekanntheitsgrad einzelner Kommunikati- } \\
\text { onsmaßnahmen mit den soziodemographischen und persönlichkeitsspezifi- } \\
\text { schen Variablen zusammenhängt. }\end{array}$ & bestätigt \\
\hline $\mathrm{H} 2$ & $\begin{array}{l}\text { Es wird angenommen, dass die Nutzungsintensität einzelner Kommunikati- } \\
\text { onsmaßnahmen mit den soziodemographischen und persönlichkeitsspezifi- } \\
\text { schen Variablen zusammenhängt. }\end{array}$ & bestätigt \\
\hline $\mathrm{H} 3$ & $\begin{array}{l}\text { Es wird angenommen, dass die Bewertung einzelner Kommunikationsmaß- } \\
\text { nahmen mit den soziodemographischen und persönlichkeitsspezifischen Vari- } \\
\text { ablen zusammenhängt. }\end{array}$ & bestätigt \\
\hline $\mathrm{H} 4$ & $\begin{array}{l}\text { Es wird angenommen, dass das Präferenzmaß einzelner Kommunikations- } \\
\text { maßnahmen mit den soziodemographischen Variablen zusammenhängt. }\end{array}$ & bestätigt \\
\hline \multicolumn{3}{|c|}{ Teilmodell 2} \\
\hline $\mathrm{H} 5$ & $\begin{array}{l}\text { Die Entwicklungs- und Aufstiegschancen führen zu einer höheren Wahlwahr- } \\
\text { scheinlichkeit des Stellenangebotes. }\end{array}$ & bestätigt \\
\hline H6 & $\begin{array}{l}\text { Die Vereinbarkeit von Beruf und Familie bzw. Privatleben führt zu einer hö- } \\
\text { heren Wahlwahrscheinlichkeit des Stellenangebotes. }\end{array}$ & bestätigt \\
\hline $\mathrm{H} 7$ & $\begin{array}{l}\text { Die leistungsorientierte Vergütung führt zu einer höheren Wahlwahrschein- } \\
\text { lichkeit des Stellenangebotes. }\end{array}$ & verworfen \\
\hline $\mathrm{H} 8$ & $\begin{array}{l}\text { Die Arbeitsplatzsicherheit führt zu einer höheren Wahlwahrscheinlichkeit des } \\
\text { Stellenangebotes. }\end{array}$ & bestätigt \\
\hline H9 & $\begin{array}{l}\text { Das Gütesiegel „audit berufundfamilie“ führt zu einer höheren Wahlwahr- } \\
\text { scheinlichkeit des Stellenangebotes. }\end{array}$ & bestätigt \\
\hline $\mathrm{H} 10$ & $\begin{array}{l}\text { Das Gütesiegel „Top Job“ führt zu einer höheren Wahlwahrscheinlichkeit des } \\
\text { Stellenangebotes. }\end{array}$ & bestätigt \\
\hline H11 & $\begin{array}{l}\text { Die Interaktion zwischen den Entwicklungs- und Aufstiegschancen und der } \\
\text { Karriereorientierung führt zu einer höheren Wahlwahrscheinlichkeit des Stel- } \\
\text { lenangebotes. }\end{array}$ & bestätigt \\
\hline H12 & $\begin{array}{l}\text { Die Interaktion zwischen der Vereinbarkeit von Beruf und Familie bzw. Pri- } \\
\text { vatleben und der Freizeitorientierung führt zu einer höheren Wahlwahrschein- } \\
\text { lichkeit des Stellenangebotes. }\end{array}$ & bestätigt \\
\hline $\mathrm{H} 13$ & $\begin{array}{l}\text { Die Interaktion zwischen der leistungsorientierten Vergütung und der Karrie- } \\
\text { reorientierung führt zu einer höheren Wahlwahrscheinlichkeit des Stellenan- } \\
\text { gebotes. }\end{array}$ & verworfen \\
\hline
\end{tabular}




\section{Hypothese Inhaltliche Fragestellung}

Ergebnis

Teilmodell 2

\begin{tabular}{|l|l|l|}
\hline H14 & $\begin{array}{l}\text { Die Interaktion zwischen dem Gütesiegel ,audit berufundfamilie“ und der } \\
\text { Freizeitorientierung führt zu einer höheren Wahlwahrscheinlichkeit des Stel- } \\
\text { lenangebotes. }\end{array}$ & verworfen \\
\hline H15 & $\begin{array}{l}\text { Die Interaktion zwischen dem Gütesiegel „Top Job“ und der Karriereorientie- } \\
\text { rung führt zu einer höheren Wahlwahrscheinlichkeit des Stellenangebotes. }\end{array}$ & verworfen \\
\hline H16 & $\begin{array}{l}\text { Die Interaktion zwischen den Gütesiegeln und ihrem jeweiligen Bekanntheits- } \\
\text { grad führt zu einer höheren Wahlwahrscheinlichkeit des Stellenangebotes. }\end{array}$ & verworfen \\
\hline H17 & $\begin{array}{l}\text { Die Interaktion zwischen den Gütesiegeln und ihrer generellen Relevanz führt } \\
\text { zu einer höheren Wahlwahrscheinlichkeit des Stellenangebotes. }\end{array}$ & $\begin{array}{l}\text { z.T. } \\
\text { bestätigt }\end{array}$ \\
\hline
\end{tabular}

Teilmodell 3

\begin{tabular}{|c|c|c|}
\hline H18 & $\begin{array}{l}\text { Es besteht ein positiver Zusammenhang zwischen dem Branchenruf und der } \\
\text { Person-Organisation-Passung. }\end{array}$ & bestätigt \\
\hline H19 & $\begin{array}{l}\text { Es besteht ein positiver Zusammenhang zwischen dem Branchenruf und dem } \\
\text { organisationalen Prestige. }\end{array}$ & verworfen \\
\hline $\mathrm{H} 20$ & $\begin{array}{l}\text { Es besteht ein positiver Zusammenhang zwischen den wahrgenommenen } \\
\text { beruflichen Chancen auf dem Arbeitsmarkt und der subjektiv eingeschätzten } \\
\text { Erfolgswahrscheinlichkeit der Bewerbung. }\end{array}$ & bestätigt \\
\hline $\mathrm{H} 21$ & $\begin{array}{l}\text { Es besteht ein positiver Zusammenhang zwischen der Person-Organisation- } \\
\text { Passung und der Bewerbungsabsicht. }\end{array}$ & bestätigt \\
\hline $\mathrm{H} 22$ & $\begin{array}{l}\text { Es besteht ein positiver Zusammenhang zwischen dem organisationalen Pres- } \\
\text { tige und der Bewerbungsabsicht. }\end{array}$ & bestätigt \\
\hline $\mathrm{H} 23$ & $\begin{array}{l}\text { Es besteht ein positiver Zusammenhang zwischen der wahrgenommenen Ar- } \\
\text { beitgeberattraktivität und der Bewerbungsabsicht. }\end{array}$ & bestätigt \\
\hline $\mathrm{H} 24$ & $\begin{array}{l}\text { Es besteht ein positiver Zusammenhang zwischen der subjektiv eingeschätzten } \\
\text { Erfolgswahrscheinlichkeit der Bewerbung und der Bewerbungsabsicht. }\end{array}$ & bestätigt \\
\hline $\mathrm{H} 25$ & $\begin{array}{l}\text { Die wahrgenommene Arbeitgeberattraktivität mediiert die positive Beziehung } \\
\text { zwischen der Person-Organisation-Passung und der Bewerbungsabsicht (Me- } \\
\text { diatorhypothese). }\end{array}$ & bestätigt \\
\hline $\mathrm{H} 26$ & $\begin{array}{l}\text { Die wahrgenommene Arbeitgeberattraktivität mediiert die positive Beziehung } \\
\text { zwischen dem organisationalen Prestige und der Bewerbungsabsicht (Media- } \\
\text { torhypothese). }\end{array}$ & bestätigt \\
\hline $\mathrm{H} 27$ & $\begin{array}{l}\text { Das Branchenwissen mediiert die positive Beziehung zwischen dem Bran- } \\
\text { chenruf und der Person-Organisation-Passung (Mediatorhypothese). }\end{array}$ & bestätigt \\
\hline $\mathrm{H} 28$ & $\begin{array}{l}\text { Das Branchenwissen mediiert die positive Beziehung zwischen dem Bran- } \\
\text { chenruf und dem organisationalen Prestige (Mediatorhypothese). }\end{array}$ & bestätigt \\
\hline
\end{tabular}

Tab. 6-11: Zusammenfassende Darstellung der Hypothesenprüfung (Eigene Darstellung, 2012) 
KAPITEL 7

\section{Diskussion und Ausblick}

\subsection{Diskussion der Ergebnisse}

Das Hauptanliegen dieser Arbeit ist die empirische Überprüfung des Informations- und Entscheidungsverhaltens potenzieller Bewerber in mittelständischen Unternehmen der Ernährungsindustrie. Zur Untersuchung dieser Forschungsfrage wurden drei Teilmodelle konzipiert, in denen das Informationsverhalten, die präferierten Arbeitsplatzmerkmale sowie die Determinanten der Bewerbungsintention dieser Zielgruppe mithilfe ausgewählter methodischer Verfahren systematisch untersucht wurden. Im Folgenden werden die im Kapitel 6 dargestellten Ergebnisse diskutiert.

\subsubsection{Ergebnisdiskussion zum ersten Teilmodell}

Betrachtet man zunächst den Bekanntheitsgrad und die Nutzungsintensität einzelner Personalmarketingmaßnahmen auf der deskriptiven Ebene, so wird deutlich, dass hierbei beträchtliche Unterschiede bestehen (vgl. Abschnitte 6.2 und 6.3). Während Stellenanzeigen, Praktika/Werkstudententätigkeiten und Stellenangebote auf den unternehmenseigenen Websites nahezu allen Befragten bekannt sind, ist die Kenntnis von Karrierenetzwerken (z.B. Xing) und hochschuleigenen Datenbanken deutlich geringer ausgeprägt. Im Einzelnen wird dabei deutlich, dass zum einen die Abschlussart einen signifikanten Einfluss auf die Wahrscheinlichkeit hat, dass die befragten Personen bestimmte Maßnahmen kennen und auch nutzen. So weisen Masterstudierende im Vergleich zu Bachelorstudierenden erwartungskonform eine signifikant bessere Kenntnis und zugleich höhere Nutzungsintensität der verfügbaren Rekrutierungskanäle auf. Hier liegt die Vermutung nahe, dass Masterstudierende aufgrund ihres bereits absolvierten Bachelorstudiums und der damit einhergehenden Studienabschlussphase bereits häufiger mit solchen Maßnahmen konfrontiert wurden und diese auch entsprechend persönlich genutzt haben. Möglicherweise stand bei einigen Personen dieser Befragtengruppe auch die Überlegung im Raum, nach dem erfolgreich absolvierten Bachelorstudium in den Beruf einzusteigen, sodass sie zwangsläufig intensiver auf die verfügbaren Kommunikationsmedien angewiesen waren. Bemerkenswert ist zudem, dass sich die Abschlussart signifikant auf die Wahrscheinlichkeit auswirkt, mit Karrie- 
renetzwerken (z.B. Xing) vertraut zu sein und diese zum Zwecke der Arbeitgeberwahl zu verwenden. Dieses Ergebnis weist zugleich darauf hin, dass die Verbreitung solcher Plattformen mit dem Qualifizierungsniveau steigt.

Interessanterweise resultieren nur wenige signifikante Zusammenhänge zwischen dem Bekanntheitsgrad der Kommunikationsmaßnahmen und der Hochschulart der Befragten, wobei hier generell zu erwarten wäre, dass Studierende an Fachhochschulen aufgrund der im Vergleich zum universitären Studium größeren Praxisnähe auch mit den hier berücksichtigten Instrumenten des Personalmarketings vertrauter sein müssten (vgl. KANNING et al., 2009). Während die Ergebnisse für die Kontakte zu Professoren relevanter Fachrichtungen, Unternehmensexkursionen und Stellenangebote auf den unternehmenseigenen Websites erwartungskonform ausfallen, zeigt sich für Fachvorträge von Unternehmensvertreter in Lehrveranstaltungen, dass Universitätsstudierende diese mit einer höheren Wahrscheinlichkeit kennen. Zudem wird bei der Nutzungsintensität der untersuchten Maßnahmen deutlich, dass die Hochschulart erwartungskonform einen signifikanten Prädiktor für Unternehmensexkursionen, Stellenangebote auf den unternehmenseigenen Websites sowie Kontakte zu Professoren relevanter Fachrichtungen darstellt.

Auffällig ist, dass sich für die soziodemographische Variable Fachrichtung sowohl im Hinblick auf den Bekanntheitsgrad der Kommunikationsmaßnahmen als auch auf ihre Nutzungsintensität nur wenige Zusammenhänge ergeben. So ist zum einen zu beobachten, dass die Wahrscheinlichkeit, Unternehmensexkursionen zu kennen und zu nutzen, bei AEW- und LMT-Studierenden - vermutlich aufgrund ihres branchenfokussierten Studiums - höher ist als bei Studierenden der BWL. Demgegenüber ist es für die LMT-Studierenden im Vergleich zu ihren Kommilitonen aus der BWL weniger wahrscheinlich, dass sie mit Karrierenetzwerken vertraut sind, während die AEW-Studierenden dieses Medium mit einer geringeren Wahrscheinlichkeit nutzen. Aus einer solchen Ergebniskonstellation lässt sich zugleich die Schlussfolgerung ableiten, dass die Karrierenetzwerke vor allem für die i.d.R. branchenübergreifend aufgestellten BWL-Studierenden einen hohen Stellenwert einnehmen.

Darüber hinaus zeigt sich, dass die Berufsorientierungen nur einen geringen Beitrag zur Vorhersage des Bekanntheitsgrades und der Nutzungsintensität von Kommunikationsmaßnahmen leisten. Eine mögliche Erklärung für diesen Befund könnte darin liegen, dass die hier berücksichtigten Rekrutierungskanäle als Instrumente des Hochschulmarketings inzwischen sehr weit verbreitet sind, wodurch potenzielle Bewerber ungeachtet ihrer persönlichen Vorzüge bei der Stellensuche mit einer Vielzahl dieser Medien konfrontiert werden. Aus diesem hohen Verbreitungsgrad folgt zugleich eine weiterführende Erklärungsalternative: Potenzielle Bewerber verhalten sich bei der Informationsbeschaffung tendenziell weniger aktiv, sondern vielmehr passiv, wodurch auch die persönlichkeitsspezifischen Merkmale naturgemäß in geringerem Maße zum Tragen kommen (vgl. SÜss, 1996). 
Anders als erwartet fällt auch das Ergebnis für das Involvement der Befragten aus. Dieses Konstrukt wurde hier auf zwei unterschiedliche Arten gemessen. Während das persönliche Involvement direkt erfasst wurde, lag dem situativen Involvement die Überlegung zugrunde, dass es mit dem näher rückenden Studienende steigt. Die Ergebnisse verdeutlichen jedoch, dass sich weder das situative Involvement - erfasst über die bis zum Studienende verbleibende Studiendauer - noch das persönliche Involvement als signifikante Prädiktoren des Bekanntheitsgrades einzelner Kommunikationsmaßnahmen erweisen. Bei der Nutzungsintensität liegen indes zumindest zwei erwartungskonforme Ergebnisse vor, denen zufolge Unternehmenspräsentationen an Hochschulen sowie hochschuleigene Datenbanken mit näher rückendem Studienende auch mit einer höheren Wahrscheinlichkeit genutzt werden. Einschränkend ist bei diesen beiden Ergebnissen allerdings zu erwähnen, dass sie lediglich für die Befragtengruppe der Bachelorstudierenden gelten. Die Wirkung persönlichkeitsspezifischer Merkmale im Bereich der Informationsbeschaffung ist angesichts dieser empirischen Befundlage insgesamt als gering zu werten.

Wider Erwarten fällt der Beitrag des Involvements auch bei der Nützlichkeitsbewertung der Kommunikationsmaßnahmen in Form von zwei signifikanten Prädiktoren erstaunlich gering aus (vgl. Abschnitt 6.4). Bei der Informationsverarbeitung wiegt dieses Ergebnis allerdings um einiges schwerer, da das Involvement gemäß dem ELM-Modell von PETTY und CACIOPPO (1986) eine zentrale Einflussvariable der Bewertung von Kommunikationsmaßnahmen darstellt. Ein hohes Involvement sollte sich demzufolge insbesondere auf die persönlich-direkten Kommunikationskanäle, die tendenziell eine höhere Informationsgüte aufweisen, positiv auswirken. Als einziges theoriekonformes Ergebnis fungiert hierbei die Bewertung der Unternehmenspräsentationen an Hochschulen, während Stellenangebote auf der unternehmenseigenen Website eher zu den unpersönlich-indirekten Quellen zählen und aus theoretischer Perspektive somit nicht unbedingt positiv mit dem zugrunde liegenden Involvement zusammenhängen sollten. In praktischer Hinsicht erscheint es jedoch durchaus plausibel, dass Studierende insbesondere am Ende ihres Studiums konkrete Stellenangebote, auf die sie sich direkt bewerben können, als nützlich bewerten.

Hinsichtlich der Nützlichkeitsbewertung von Kommunikationsmaßnahmen erscheint es zudem überraschend, dass das Fachhochschulstudium als negativer Prädiktor bei Unternehmenspräsentationen an Hochschulen, Kontakten zu Professoren relevanter Fachrichtungen, Unternehmensexkursionen sowie Job- und Fachmessen außerhalb der Hochschule fungiert. Ein möglicher Grund für dieses Ergebnis könnte darin liegen, dass Studierende an Fachhochschulen aufgrund ihrer besonders großen Praxisnähe höhere Erwartungen an die Kommunikationsmaßnahmen stellen, denen die Unternehmen entsprechend auch schwieriger nachkommen können.

Während die Variable Abschlussart die Bewertung von Unternehmenspräsentationen an Hochschulen und Unternehmensexkursionen positiv beeinflusst, ergibt sich für die Unterstützung 
von Projekt- und Abschlussarbeiten ein gegenläufiges Ergebnis. Dieses könnte darauf zurückzuführen sein, dass Bachelorstudierende gerade mit der letztgenannten Maßnahme bislang nur wenige eigene Erfahrungen sammeln konnten und diese aufgrund der zumindest teilweise fehlenden Bewertungsgrundlage negativer beurteilen.

Erwartungskonform fällt hingegen das Ergebnis für die beiden Berufsorientierungen - Karriereorientierung und alternatives Engagement - aus, die sich als signifikante Prädiktoren der Nützlichkeitsbewertung von mehreren Kommunikationsmaßnahmen erweisen. Während bei diesen beiden Berufsorientierungen die beruflichen Ziele, wenngleich strukturell unterschiedlich ausgeprägt, im Vordergrund stehen, schätzen freizeitorientierte Personen eher außerberufliche Aktivitäten. Insofern erscheint es evident, dass sich gerade die zuerst genannten Merkmale signifikant auf die Vorhersage der Nützlichkeitsbewertung auswirken. Bemerkenswert und von praktischer Bedeutung für die externe Ansprache potenzieller Bewerber ist zudem, dass diese persönlichkeitsspezifischen Merkmale nach Berücksichtigung der soziodemographischen Variablen einen zusätzlichen Beitrag zur Vorhersage der Maßnahmenbewertung ermöglichen und somit ein relevantes Segmentierungskriterium darstellen (MEFFERT et al., 2012).

Für das Präferenzmaß der einzelnen Kommunikationsmaßnahmen geht schließlich hervor, dass hier zwar signifikante, aber in ihrer Stärke nur sehr geringe Zusammenhänge mit soziodemographischen Merkmalen der Befragten hervorgehen, deren Aussagekraft folglich als ausgesprochen eingeschränkt zu werten ist (vgl. Abschnitt 6.5).

Abschließend ist gleichwohl zu konstatieren, dass die Vorhersage der hier betrachteten Kriterien anhand der Modellvariablen nur in begrenztem Maße gelungen ist. In den entsprechenden Regressionsmodellen fallen die Effektstärken mit ihren zugrunde liegenden Gütemaßen, Nagelkerkes $\mathrm{R}^{2}$ in der logistischen Regression bzw. dem Bestimmtheitsmaß $\mathrm{R}^{2}$ in der multiplen Regression, relativ gering aus, wodurch ein erheblicher Varianzanteil unerklärt bleibt. Dennoch scheint, wie die multiplen Regressionsanalysen andeuten, eine nach soziodemographischen und persönlichkeitsspezifischen Segmentierungsmerkmalen aufgeteilte Betrachtung von potenziellen Bewerbern sinnvoll zu sein. Vor allem den beiden Berufsorientierungen - Karriereorientierung und alternatives Engagement - könnte eine besondere Bedeutung bei der Identifikation unterschiedlicher Zielgruppensegmente auf dem externen Bewerbermarkt zukommen.

\subsubsection{Ergebnisdiskussion zum zweiten Teilmodell}

Zentrales Forschungsziel des zweiten Teilmodells besteht darin, eine fundierte methodische Alternative zur unmittelbaren Erhebung der Wichtigkeitsurteile einzelner Arbeitsplatzmerkmale aufzuzeigen, da sich diese Herangehensweise aufgrund von systematischen Verzerrungsten- 
denzen zunehmend als ungeeignet erwiesen hat (vgl. Abschnitt 2.5.1). Dazu wurde mithilfe der DCA der Einfluss von insgesamt fünf Arbeitsplatzmerkmalen auf die Auswahlentscheidung von Stellenangeboten bei potenziellen Bewerbern untersucht. Die daraus resultierenden Befunde bestätigen einerseits die Ergebnisse direkter Befragungen, in denen den Entwicklungs- und Aufstiegschancen sowie der Vereinbarkeit von Beruf und Familie bzw. Privatleben eine große Bedeutung beigemessen wird (z.B. GROBE, 2003; LEWANDOWSKI und LIEBIG, 2004; KIRCHGEORG und GÜNTHER, 2006; BOURHIS und MEKKAOUI, 2010). Hinsichtlich der Arbeitsplatzsicherheit stehen sie dazu aber im Widerspruch, da die meisten Studien diesem Arbeitsplatzmerkmal einen mittleren bis geringen Stellenwert einräumen. Anders als bei der Verwendung direkter Befragungsmethoden zeigt sich hierbei, dass potenzielle Bewerber dieses Arbeitsplatzmerkmal als besonders gewichtig einschätzen. Eine mögliche Erklärung könnte darin liegen, dass befristete Arbeitsverhältnisse gerade unter Berufseinsteigern am weitesten verbreitet sind und diese Zielgruppe somit am meisten betreffen. Dabei könnte die entsprechende mediale Berichterstattung - Stichwort „Generation Probezeit“ - massiv dazu beitragen, dass unbefristete Arbeitsverträge inzwischen eher als Ausnahme betrachtet werden und deshalb eine übergeordnete Rolle bei potenziellen Bewerbern einnehmen (vgl. z.B. HAMBURGER ABENDBLATT, 2012; SPIEGEL ONLINE, 2013).

Entgegen bisheriger Befunde fällt auch das Ergebnis für das Arbeitsplatzmerkmal Bezahlung aus. Während das Einkommen bei seiner direkten Bedeutsamkeitseinschätzung überwiegend im mittleren oder gar im unteren Bereich angesiedelt wird, deuten die Ergebnisse aus dekompositionellen Verfahren eher auf einen hohen Stellenwert dieses Merkmals hin (vgl. Abschnitt 2.5.1). Aufgrund stark abweichender Operationalisierungen sind die Ergebnisse dieser Untersuchungen allerdings kaum miteinander vergleichbar. In dieser Studie konnte indes gezeigt werden, dass die leistungsorientierte Vergütung zu einem geringeren Nutzen des Stellenangebotes führt und folglich seine Wahlwahrscheinlichkeit mindert. Diese Vergütungsart ist generell dadurch gekennzeichnet, dass hiermit einerseits ein höheres Einkommen erzielt werden kann (STOCK-HOMBURG, 2010). Gleichzeitig steigt damit jedoch auch das sog. Vergütungsrisiko, was seinerseits anhand von drei Faktoren - Variabilität, Verlustrisiko sowie Fristigkeit der Vergütung - beschrieben werden kann (TUSCHKE, 2011).

Der oben dargestellte Befund lässt sich möglicherweise darauf zurückführen, dass es sich bei den Befragten um zukünftige Berufseinsteiger handelt, die bis dato nur wenige Gelegenheiten hatten, ihre eigene Leistungsfähigkeit im Berufsleben unter Beweis zu stellen. Dadurch fehlen ihnen im Sinne der Selbstwirksamkeit möglicherweise noch positive Überzeugungen, die in den zugrunde liegenden beruflichen Situationen erforderlich wären (vgl. HECKHAUSEN und HECKHAUSEN, 2010). Neben dieser lernpsychologischen Erklärung lässt sich mithilfe der VIETheorie auch eine motivationspsychologische Interpretation des obigen Befundes anbringen (vgl. Abschnitt 3.2). So nimmt THIERRY (2001) zufolge die Instrumentalität einer leistungsori- 
entierten Vergütung mit dem dazugehörigen Vergütungsrisiko ab, da potenzielle Bewerber die hierfür zu erbringenden Leistungen nicht zuverlässig beurteilen können und die entsprechenden Handlungsfolgen im Vorfeld als unsicher einschätzen. Demgegenüber ist über ein festes Gehalt sichergestellt, dass das erwartete Handlungsergebnis mit einer sehr hohen Wahrscheinlichkeit eintrifft, wenn die vorher vertraglich festgelegten Anforderungen, die ihrerseits nicht unmittelbar von der Leistung bzw. vom Erfolg anhängen, erfüllt werden. Vor diesem Hintergrund ist ferner anzunehmen, dass auch die Valenz der variablen Vergütung im Vergleich zu festen Gehaltsanteilen geringer ausfällt. Insofern erscheint es schlüssig, dass potenzielle Bewerber in dieser Situation ein durchschnittliches Festeinkommen präferieren. Schließlich weist TUSCHKE (2011) darauf hin, dass variable leistungsabhängige Vergütungsanteile auch deshalb zu Fehlanreizen führen können, da sie die im Vorfeld bestehende intrinsische Motivation potenzieller Bewerber abschwächen können. Dieses Phänomen wird als Korrumpierungseffekt bezeichnet und hinsichtlich seiner ökologischen Validität in der Literatur inzwischen auch sehr kritisch diskutiert (RYAN und DECI, 2000; RHEINBERG, 2010). THEUVSEN (2003) zufolge sollte ein solcher Effekt im Zusammenhang mit einer leistungsorientierten Vergütung insbesondere dann auftreten, wenn potenzielle Bewerber dadurch ihre Autonomie eingeschränkt sehen und zugleich einen höheren Grad an sozialer Kontrolle erleben. Davon zeigen sich vor allem Personen betroffen, die monetären Anreizen eine untergeordnete Bedeutung beimessen, wozu in dieser Studie die freizeitorientierten und alternativ engagierten Befragten zählen.

Alternative Interpretationsmöglichkeiten für diesen empirischen Befund ergeben sich auch aus betriebswirtschaftlichen Theorien (TUSCHKE, 2011). Einen Ansatzpunkt hierfür stellt die Prinzipal-Agent-Theorie dar, in der postuliert wird, dass sowohl Unternehmen als auch Arbeitnehmer nach dem Prinzip der Nutzenmaximierung handeln, wobei die Unternehmen ihre Mitarbeiter aufgrund von asymmetrischer Informationsverteilung häufig nur schwer beurteilen können, was letzteren wiederum opportunistisches Verhalten ermöglicht (WISEMAN et al., 2000). Um diesen Spielraum zu begrenzen, werden hohe variable Vergütungsanteile empfohlen. Damit gehen allerdings auch unterschiedliche Risikopräferenzen aufseiten der Unternehmen und der Arbeitnehmer einher. Demzufolge löst eine leistungsabhängige variable Vergütung bei einem (potenziellen) Mitarbeiter grundsätzlich eine risikoaverse Haltung aus, da dieser seine möglichen Einkommensverluste weniger gut absichern kann als ein ganzes Unternehmen, das seine Risiken streuen und dadurch zugleich besser kontrollieren kann (TUSCHKE, 2011). Demzufolge sollten sich Personen mit einer hohen Risikoaversion stets für diejenige Alternative entscheiden, die beim gleichen ökonomischen Wert ihre Unsicherheit minimiert.

Ein im Vergleich dazu differenzierteres Interpretationsmuster bietet die Prospect Theorie von KAHNEMAN und TVERSKY (1979), der zufolge sich Risikopräferenzen in Abhängigkeit von der Erreichbarkeit von Zielen ändern können. Die Wahl einer Alternative wird dabei von dem subjektiven Wert ihrer Konsequenzen und der davon unabhängigen Gewichtung der Eintre- 
tenswahrscheinlichkeit bestimmt. Der daraus resultierenden Wertfunktion liegen drei zentrale Aspekte zugrunde: Referenzpunktabhängigkeit, unterschiedliche Gewichtung von Gewinnen und Verlusten sowie verminderte Sensitivität (vgl. Holling und MELlES, 2004). Demzufolge wird der subjektive Wert der Konsequenzen nicht durch seine absolute Bedeutung eingeschätzt, sondern erfolgt in Relation zu einem neutralen Referenzpunkt, der als angemessen betrachtet wird. Die Konsequenzen werden entsprechend ihrer relativen Position zu einem solchen Referenzpunkt als Gewinne oder Verluste beurteilt. Hierbei werden die Verluste zugleich stärker gewichtet als damit korrespondierende Gewinne. Diese asymmetrische Bewertung wird innerhalb der Prospect Theorie mit dem ungleichen Erleben der Aversität von Verlusten gegenüber der Attraktivität von Gewinnen erklärt. Hinter der verminderten Sensitivität verbirgt sich schließlich die Beobachtung, dass mit steigenden Gewinnen oder Verlusten der Wertzuwachs abnimmt. Die bewerteten Konsequenzen werden durch eine Funktion der Eintrittswahrscheinlichkeit gewichtet, wobei geringe Wahrscheinlichkeiten tendenziell übergewichtet, während mittlere und hohe eher untergewichtet werden. Vor diesem theoretischen Hintergrund könnte das überwiegend risikoaverse Entscheidungsverhalten der Befragten dadurch erklärt werden, dass sie aufgrund des gewählten Referenzpunktes ein festes Gehalt ohne variable leistungsabhängige Bestandteile als eine vergleichsweise sichere Gewinnmöglichkeit beurteilen und sich deshalb eher für diese Vergütungsstruktur entscheiden (vgl. WISEMAN et al., 2000). Eine weitere Erklärungsmöglichkeit für den obigen Befund ist schließlich darin zu sehen, dass die mit einer leistungsorientierten Vergütung einhergehenden Vorteile innerhalb des Wahlexperimentes schlichtweg nicht ausreichend bekannt waren und diese Vergütungsform auch deshalb zu einem geringeren Nutzen des Stellenangebotes führte. Um diese Erklärungsalternative ausschließen zu können, sollte in zukünftigen experimentellen Studien eine kurze Erläuterung zu den Rahmenbedingungen der variablen Vergütung erfolgen.

Bemerkenswert ist an dieser Stelle das Schätzergebnis des Interaktionseffektes, aus dem hervorgeht, dass sich die leistungsorientierte Vergütung bei karriereorientierten Befragten nicht länger negativ auswirkt. Dieser theoriekonforme Befund weist zugleich darauf hin, dass der Einfluss von Arbeitsplatzmerkmalen auf die Auswahlentscheidung von Stellenangeboten differenziell, und zwar in Abhängigkeit der Berufsorientierungen potenzieller Bewerber zu betrachten ist. Zwei weitere Schätzergebnisse untermauern die theoretische Kernannahme des Modells der Person-Organisation-Passung, der zufolge potenzielle Bewerber sich für solche Arbeitgeber entscheiden, deren wahrgenommene Arbeitsplatzmerkmale am meisten mit ihrem eigenen Selbstbild, hier in Gestalt von Berufsorientierungen, übereinstimmen (KRISTOF, 1996). So beeinflusst zum einen die Vereinbarkeit von Beruf und Familie bzw. Privatleben signifikant die Auswahlentscheidung von freizeitorientierten Personen. Das zweite erwartungskonforme Ergebnis ist im signifikanten Interaktionsterm aus Karriereorientierung und Entwicklungs- und Aufstiegschancen zu sehen. 
Gütesiegel haben im Bereich des Personalmarketings primär die Aufgabe, die bestehenden Unsicherheiten bei potenziellen Bewerbern zu reduzieren und ihre Entscheidung, sich bei einem bestimmten Unternehmen zu bewerben, positiv zu beeinflussen (vgl. HUF, 2007: TAVAKKOLI, 2009; SCHERM und SÜSS, 2010; NAUNDORF und SPENGLER, 2012). Betrachtet man die postulierte unsicherheitsreduzierende Wirkung von Gütesiegeln aus der informationsökonomischen Perspektive, dann besteht die Ausgangsfrage hierbei darin, inwieweit die beiden untersuchten Gütesiegel die Erfahrungs- und Vertrauenseigenschaften eines Stellenangebotes in Sucheigenschaften transformieren und somit als verlässliches Signal für nicht beobachtbare Arbeitsplatzmerkmale genutzt werden können (vgl. SCHMIDTKE, 2002). Dabei sollte ist es mithilfe der Gütesiegel, die von anbieterunabhängigen und zugleich vertrauenswürdigen Instanzen nach vorher festgelegten Prüfkriterien vergeben werden, auch grundsätzlich möglich sein, bisher fehlendes Vertrauen in den Arbeitgeber aufzubauen und zugleich eine positive Unternehmensreputation zu erschaffen (NOLL und WINKLER, 2004). Ihre tatsächliche Wirkung als unsicherheitsreduzierendes Signal hängt zugleich von mehreren weiterführenden Prämissen ab (LICHARZ, 2002, zit. in NOLL und WINKLER, 2004). So ist es zum einen ganz entscheidend, ob die Zertifizierungsorganisation oder das Gütesiegel bekannt sind. Zudem stellt sich die Frage, ob und inwieweit die Zertifizierung bzw. Auszeichnung durch Transparenz und Neutralität bzw. fehlende kommerzielle Interessen gekennzeichnet ist. Darüber hinaus kann die Orientierungsfunktion eines Gütesiegels nur dann erfüllt werden, wenn die Anzahl von Siegel-Anbietern für den Konsumenten überschaubar ist und die Siegel-Vergabe nach standardisierten Kriterien erfolgt. Schließlich wird eine regelmäßige Überprüfung der bereits vergebenen Gütesiegel vorausgesetzt, um die damit einhergehende Informationsfunktion möglichst aktuell zu halten.

Die deskriptiven Ergebnisse bestätigen zunächst, dass die Gütesiegel ihrer angenommen Wirkung gerecht zu werden vermögen. So gab rund die Hälfte der Befragten an, dass sie von der grundsätzlichen Relevanz des Gütesiegels als wichtiges oder sehr wichtiges Entscheidungskriterium überzeugt sind. Diese deskriptiven Ergebnisse werden von den Schätzergebnissen der DCA jedoch nur z.T. bestätigt. So führt der einem Gütesiegel generell beigemessene Stellenwert nur im Falle des ,audit berufundfamilie“ zu einer höheren Wahlwahrscheinlichkeit des Stellenangebotes, während dieser Effekt beim Gütezeichen „Top Job“ ausbleibt.

Betrachtet man hingegen den Bekanntheitsgrad der beiden hier berücksichtigten Gütesiegel, so ist dieser mit knapp einem Fünftel der Befragten, denen das jeweilige Gütezeichen bereits vor der Befragung ein Begriff war, als gering einzustufen. Aus der Modellschätzung geht hierbei hervor, dass der Bekanntheitsgrad bei den dazugehörigen Siegeln unterschiedlich wirkt. Während „Top Job“ im Falle seiner Bekanntheit keinen signifikanten Einfluss ausübt, führt „,audit berufundfamilie“"zu einer geringeren Wahlwahrscheinlichkeit des Stellenangebotes. Anders als erwartet, führen beide Gütesiegel solange zu einem größeren Nutzen des Stellenangebotes, wie sie potenziellen Bewerbern noch nicht bekannt sind. Die divergente Wirkung des Bekanntheits- 
grades lässt vermuten, dass insbesondere der oben dargestellte Interessenskonflikt zwischen kommerziellen Anbieterzielen und der Vergabe von Gütezeichen für den ausbleibenden positiven bzw. negativen Effekt ursächlich sein könnte. Ein solcher Interessenskonflikt vermag nämlich nur dann ersichtlich zu werden, wenn man sich intensiver mit dem Aussagewert von Gütesiegeln befasst, was wiederum auf die Bedingung mit dem vorliegenden Bekanntheitsgrad zutrifft (vgl. NAUNDORF und SPENGLER, 2012). Ebenso ist anzunehmen, dass die angestrebte Informationsfunktion des Gütesiegels aufgrund der großen Menge an Anbietern, die sich wegen der fehlenden Standardisierung in ihrem Qualitätslevel nur sehr bedingt miteinander vergleichen lassen, unterlaufen wird (vgl. SPILLER, 2001). Insgesamt wird somit deutlich, dass die beiden Gütesiegel ihrem eigentlichen Wirkprinzip als Vertrauenssignale zumindest bislang nicht gerecht werden. Während sie aufgrund ihres derzeit geringen Bekanntheitsgrades einen höheren Nutzen der Stellenangebote bewirken, wird ihre weitere Verbreitung zwangsläufig dazu führen, dass auch die hier beobachteten positiven Effekte zurückgehen. Die Frage, ob und inwieweit Gütesiegel mittel- und langfristig das Vertrauen in mittelständische Unternehmen als Arbeitgeber stärken und ihre Reputation verbessern, kann anhand der erhobenen Daten indes nicht hinreichend beantwortet werden und verlangt deshalb weiterführende Studien auf diesem Gebiet.

Eine weitere Forschungsfrage innerhalb dieses Teilmodells bestand darin, ob Gütesiegel neben einer reinen Informationsfunktion auch eine Wertfunktion, die sich beispielsweise im Sinne einer Unternehmenskultur niederschlägt, vermitteln. Demzufolge sollten sich eher diejenigen Befragten für das Stellenangebot entscheiden, deren Berufsorientierungen mit den im Gütesiegel kommunizierten Werten übereinstimmen. Die Schätzergebnisse zeigen allerdings, dass die beiden Parameter der zugrunde liegenden Interaktionseffekte nicht signifikant von null verschieden sind und somit den Nutzen des Stellenangebotes nicht bedeutsam beeinflussen. Eine mögliche Erklärung für diesen Befund könnte darin liegen, dass die beiden Gütesiegel die erforderlichen Wertaussagen nicht genügend transportieren und dadurch keine ausreichende Identifikationsgrundlage für die unterschiedlichen Berufsorientierungen potenzieller Bewerber bieten.

Aus methodischer Sicht lässt sich bei diesem Teilmodell schlussfolgern, dass die DCA ein geeignetes Verfahren darstellt, um die mit einer direkten Befragung einhergehenden Probleme bei der Bedeutsamkeitseinschätzung von Arbeitsplatzmerkmalen zu vermeiden (vgl. SATTLER, 2006). Demzufolge führt die direkte Präferenzbefragung zu systematischen Verzerrungen der Bedeutungsgewichte, wobei wichtige Merkmale unterschätzt, während bedeutsame Attribute gleichzeitig überbewertet werden (TEICHERT, 2000). Hinzu kommt, dass diese Verzerrungstendenz bei bestimmten Arbeitsplatzmerkmalen, insbesondere beim Faktor Bezahlung, durch sozial erwünschtes Antwortverhalten intensiviert wird (vgl. Abschnitt. 2.5.1). So hat die Untersuchung von WiLTINGER (1997) gezeigt, dass Befragte gerade bei diesem Attribut bestrebt sind, 
ein positives Bild von sich abzugeben, wodurch sein Stellenwert deutlich unterschätzt wird. Demgegenüber war es mithilfe der DCA möglich, höhere Validitätswerte zu erzielen. So wurden zur Sicherstellung der internen Validität mögliche Einflüsse der Präsentationsreihenfolge mittels einer teilweisen Permutation kontrolliert, indem sowohl absolute als auch relative Positionseffekte der Arbeitsplatzmerkmale ausbalanciert wurden. Ein hohes Involvement der Befragten bei diesem Entscheidungsgegenstand wurde dabei über die Stichprobenauswahl sichergestellt, da ausschließlich Studierende in höheren Semestern befragt wurden, die somit kurz vor ihrer Bewerbungsphase standen. Bei der externen Validität ist es indes ausschlaggebend, einen möglichst hohen Realitätsbezug der Wahlaufgaben zu schaffen, was über die praxisbezogenen Formulierungen der Arbeitsplatzmerkmale bewerkstelligt wurde. Und nicht zuletzt aufgrund der reduzierten sozialen Erwünschtheitseffekte hat sich die DCA als ein valideres Instrument zur Modellierung von Arbeitgeberwahlverhalten erwiesen (HARTMANN und SATTLER, 2004). Durch eine Kombination von Arbeitsplatzmerkmalen und den Berufsorientierungen im Schätzmodell wurden mit dem hier eingesetzten Verfahren zudem Interaktionseffekte analysiert, aus denen zugleich deutlich spezifischere Aussagen für die Praxis gewonnen werden können. Insgesamt lässt sich festhalten, dass die DCA tiefgehende Informationen zum Gewicht einzelner Entscheidungsfaktoren bei der Arbeitgeberwahl liefert und das Verfahren somit auch auf Handlungsentscheidungen, die sich nicht ausschließlich auf Marktgüter im engeren Sinne beziehen, anwendbar ist (vgl. AUSPURG und LIEBE, 2011).

\subsubsection{Ergebnisdiskussion zum dritten Teilmodell}

Das dritte Teilmodell fokussiert darauf, den prädiktiven Beitrag einzelner theoriegeleitet ausgewählter Prädiktoren zur Bewerbungsintention zu ermitteln. Entsprechend der dazu aufgestellten Hypothesen wurde ein Kausalmodell entwickelt und mithilfe einer PLS-Analyse statistisch überprüft. Insgesamt ist es damit gelungen, die postulierten positiven Wirkungen einzelner Variablen auf die Bewerbungsabsicht in mittelständischen Unternehmen der Ernährungsindustrie empirisch zu bestätigen und weiter zu differenzieren. Die Bewerbungsabsicht wird dabei substanziell durch die latenten Variablen Erfolgswahrscheinlichkeit der Bewerbung, organisationales Prestige, Person-Organisation-Passung sowie wahrgenommene Arbeitgeberattraktivität erklärt, wobei die zuletzt genannte Variable den mit Abstand höchsten prädiktiven Beitrag leistet. Die Originalität dieses Teilmodells ist zugleich in mehreren weiteren Punkten zu sehen: So wird zunächst deutlich, dass der Branchenruf der Ernährungsindustrie bei der Analyse des Entscheidungsverhaltens potenzieller Bewerber einen hohen Stellenwert einnimmt, indem er seinerseits zwei proximale Variablen der Bewerbungsabsicht - das organisationale Prestige und die Person-Organisation-Passung - direkt oder indirekt beeinflusst. HAUTZINGER (2009) hatte in seiner Studie bereits positive Effekte des Branchenrufs nachgewiesen, diese Untersuchung spezifiziert seine Befunde nun gezielt für die Ernährungsindustrie. Außerdem wird anhand des 
hier aufgestellten Kausalmodell deutlich, dass einzelne positive Effekte durch die beiden Variablen wahrgenommene Arbeitgeberattraktivität und Branchenwissen vermittelt werden. Eine explizite Analyse der mediierten Wirkbeziehungen führt wiederum dazu, dass die Gültigkeit der Modellschätzung erhöht wird, was zugleich die inhaltliche Interpretation der Ergebnisse erleichtert. Die hier erzielten Ergebnisse verdeutlichen zugleich, dass das Branchenwissen, das im Kontext der Arbeitgeberwahl bislang kaum berücksichtigt wurde, für das Verständnis des Entscheidungsverhaltens potenzieller Bewerber von zentraler Bedeutung ist. Die nachgewiesenen Mediationseffekte unterstreichen dabei, wie wichtig der zugrunde liegende Wissenserwerb, der sowohl vonseiten der mittelständischen Unternehmen als auch der einschlägigen Branchenverbände der Ernährungsindustrie gefördert werden kann, für die Gewinnung neuer Mitarbeiter ist. Unter dem Gesichtspunkt der Suffizienz der TOPB wird ferner nachgewiesen, dass die Erweiterung des Grundmodells um die Determinante Person-Organisation-Passung zu einer verbesserten Vorhersage der Kriteriumsvariable Bewerbungsintention beiträgt und daher als angemessen zu werten ist.

Aus methodischer Sicht stellt zudem die grundsätzliche Eignung der PLS-Analyse zur Erklärung der Bewerbungsabsicht eine diskussionswürdige Frage dar. Im Gegensatz zur Kovarianzstrukturanalyse, die von der Richtigkeit des spezifizierten Modells ausgeht und dazu die vom Modell generierte Kovarianzmatrix mit der empirisch ermittelten Kovarianzmatrix abgleicht, werden die Parameter für das PLS-Modell so geschätzt, dass sie die beste Vorhersage leisten und dazu die Rohdatenmatrix möglichst optimal reproduzieren (HAIR et al., 2011). Es stellt sich innerhalb der PLS-Analyse somit weniger die Frage, ob ein Modell wahr oder falsch ist, sondern vielmehr ob es über mehr oder weniger Erklärungskraft verfügt (vgl. BOSSOW-THIES und PANTEN, 2009). Infolgedessen ist die PLS-Analyse nicht zum Theorietest geeignet, während sie hinsichtlich ihrer Erklärungs- bzw. Prognosekraft der Kovarianzstrukturanalyse überlegen ist (REINARTZ et al., 2009). Während in der Kovarianzstrukturanalyse primär die globalen Fitindizes als Bewertungsgrundlage der Gesamtmodellgüte dienen, werden in der PLS-Analyse die lokalen Fitindizes in Form des Bestimmtheitsmaßes $\mathrm{R}^{2}$ zur Ergebnisinterpretation mit dem Ziel herangezogen, die relevanten endogenen Variablen möglichst gut zu erklären (HAIR et al., 2011). Beleuchtet man diese forschungsmethodische Divergenz etwas genauer, so ist hierbei zu hinterfragen, ob eine reine Theorieprüfung anhand eines Modells ohne eine damit zugleich einhergehende Erklärungskraft wirklich erkenntnisreich ist, oder ob eine zusammenführende Betrachtung dieser beiden Forschungsziele sowohl aus der marketingwissenschaftlichen als auch aus der anwendungsbezogenen Perspektive vorteilhafter wäre (FASSOTT, 2005). Für die PLS-Analyse sprechen in diesem Sinne erste empirische Vergleichsuntersuchungen der beiden Schätzmethoden, aus denen hervorgeht, dass der Einsatz von PLS nicht ausschließlich auf explorative Forschungszwecke beschränkt bleiben muss, sondern durchaus auch als konfirmatorisches Analyseinstrument geeignet ist (HAIR et al., 2012; HENSELER und SARSTEDT, 2012). Wenn das Erkenntnisziel einer Untersuchung hingegen in der Hypothesenprüfung auf der Ebene 
von Wirkbeziehungen zwischen zwei latenten Variablen anzusiedeln ist, dann stellt die PLSAnalyse gar ein konservativeres Verfahren dar, da es die Pfadkoeffizienten im Strukturmodell niedriger schätzt als das kovarianzbasierte Verfahren LISREL (FASSOTT, 2005). Insgesamt lässt sich in diesem Kontext daher konstatieren, dass das zentrale Forschungsziel, nämlich die empirische Überprüfung eines Erklärungsmodells zur Bewerbungsabsicht in der Ernährungsindustrie, mittels der hier gewählten methodischen Vorgehensweise erreicht werden konnte.

\subsection{Limitationen und weiterer Forschungsbedarf}

Die Ergebnisse dieser Arbeit müssen vor dem Hintergrund einiger Einschränkungen interpretiert werden. Die erste Limitation betrifft die Analyse des Informationsverhaltens potenzieller Bewerber. So wurden hier ausschließlich formelle Methoden der externen Bewerberansprache bewertet, während informelle Kanäle, wie beispielsweise Mitarbeiterempfehlungen oder die „Mund-zu-Mund-Propaganda“, unberücksichtigt blieben. Diese einseitige Auswahl von Kommunikationsmaßnahmen lässt sich zugleich damit begründen, dass formelle Ausschreibungen häufig aufgrund von Betriebsvereinbarungen oder anderen unternehmensinternen Richtlinien vorgeschrieben werden und in der Unternehmenspraxis deshalb am weitesten verbreitet sind. Andererseits können informelle Rekrutierungswege zu geeigneteren Bewerbungen führen, indem sie eine strengere Vorauswahl durch die aktuellen Mitarbeiter ermöglichen (vgl. MARCUS, 2011). Künftige Studien könnten sich somit gezielt der Frage widmen, welche systematischen Unterschiede bei diesen beiden Formen der externen Bewerberansprache vorzufinden sind.

Aufgrund der bislang kaum existierenden theoretische Annahmen war es im Vorfeld zudem nicht möglich, begründete Hypothesen darüber abzuleiten, welche soziodemographischen und persönlichkeitsspezifischen Merkmale das Informationsverhalten potenzieller Bewerber beeinflussen. Dementsprechend standen hier zunächst explorative Überlegungen im Vordergrund, die ihrerseits empirisch gestützt bzw. falsifiziert wurden. Die hier erzielten Ergebnisse sollten in weiterführenden Studien dazu genutzt werden, die Theorienbildung innerhalb dieser Forschungsfrage voranzubringen.

Betrachtet man zudem die Wirksamkeit der einzelnen Rekrutierungsquellen, so beschränkt sich die hier durchgeführte Analyse darauf, ob und inwieweit sie die potenziellen Bewerber überhaupt erreichen. Zukünftige Studien könnten sich indes mit der weiterführenden Frage befassen, welche der hier dargestellten Maßnahmen faktisch zu einer Bewerbung führen und ob es sich dabei um ein einzelnes Medium oder um eine Kombination aus mehreren Maßnahmen handelt. Auch wäre es interessant zu erfahren, welche Spezifität und Informationsmenge bei den einzelnen Maßnahmen von den Bewerbern bevorzugt wird. 
Aus prozessualer Sicht liegt eine weitere Einschränkung dieser Studie darin, dass hier lediglich auf die erste Phase des Personalmarketings, in der aus potenziellen Bewerbern tatsächliche werden, fokussiert wurde. Aus der Bewerberperspektive sind diesem Prozess entsprechend bis zum Eintritt in eine Organisation jedoch noch weitere aufeinander bauende Entscheidungen zu treffen (vgl. MOSER und ZEMPEL, 2006). Eine wesentliche Konsequenz für künftige Studien besteht daher darin, die in den einzelnen Phasen zu treffenden Entscheidungen von Bewerbern vorrangig unter der Annahme eines prozessualen Verlaufs zu untersuchen. So stellt sich hier beispielsweise die Frage, ob und inwieweit sich die in dieser Studie als einflussreich erkannten Arbeitsplatzmerkmale auch in anderen Phasen des Personalmarketings als einflussreich erweisen (vgl. BoswelL et al., 2003). Dabei sollten auch andere Arbeitsplatzmerkmale betrachtet werden. Analog dazu wäre in zukünftigen Studien eine vergleichende Analyse der vielen übrigen derzeit auf dem Markt verfügbaren Employer Awards bzw. der dazugehörigen Gütesiegel erstrebenswert (vgl. Abschnitt. 2.5.2).

In diesem Zusammenhang müsste zugleich die Frage gestellt werden, ob eine standardisierte schriftliche Befragung als alleinige Erhebungsmethode geeignet ist, um den gesamten Entscheidungsprozess zu untersuchen, oder ob andere methodischer Zugänge für bestimmte Forschungsfragen in diesem Bereich zielführender wären. An erster Stelle können hier qualitative Interviews genannt werden, aus denen sich ableiten ließe, ob und inwieweit sich die entscheidungsrelevanten Erwartungen der Bewerber aufgrund der im Auswahlprozess hinzu gewonnen Informationen bzw. Erfahrungen verändern und wie Unternehmen auf die zugrunde liegenden Entscheidungen einwirken. Um solche Entscheidungsprozesse möglichst präzise abzubilden, wäre zudem anstelle des hier verwendeten Querschnittdesigns eine Längsschnittstudie, in der Bewerber zu mehreren Erhebungszeitpunkten befragt werden, zu konzipieren.

Bei der Erhebung von Wichtigkeitsurteilen mittels dekompositioneller Verfahren, wie der hier eingesetzten DCA, wäre es außerdem sinnvoll, die differenzielle Wirkung von Arbeitsplatzmerkmalen tiefergehender zu erforschen. In diesem Rahmen könnte beispielsweise neben den Berufsorientierungen auch der Einfluss weiterer persönlichkeitsspezifischer Variablen, wie beispielsweise der Selbstwirksamkeitserwartung potenzieller Bewerber, ermittelt werden.

Obwohl das Kausalmodell zur Bewerbungsintention mit sehr zufriedenstellenden Gütemaßen einhergeht, legt es zugleich offen, dass auch andere, hier nicht berücksichtigte Determinanten bedeutsam sein könnten. Infolgedessen könnte in zukünftigen Studien eine noch höhere Varianzaufklärung erzielt werden, indem der Einfluss weiterer Prädiktoren systematisch untersucht wird. Hierbei sind insbesondere die übrigen Komponenten des Rahmenmodells zur PersonUmwelt-Passung, wie beispielsweise die Person-Job-Passung oder die Person-Kollegen-Passung, zu nennen (KRISTOF, 1996). Zudem könnten auch andere Erweiterungsvorschläge zur Theorie des geplanten Verhaltens, wie sie unter anderem von JONAS und DOLL (1996) vor- 
gelegt wurden, berücksichtigt werden. So erscheint es beispielsweise sinnvoll, die subjektive Norm differenzierter zu betrachten und dabei auch weitere unterschiedliche normative Einflüsse, wozu neben der persönlichen Norm auch die soziale Norm und die persönliche Norm im Sinne der Selbstkonsistenz zählen, zu erfassen. Ebenso wäre im Sinne der Suffizienz zu prüfen, ob die eigenständige Erfassung der antizipierten Emotion Stolz, die bei potenziellen Bewerber hinsichtlich eines bestimmten Arbeitgebers aufkommt, zu einer höheren Prognosekraft des Modells führt (vgl. HECKHAUSEN und HECKHAUSEN, 2010).

Schließlich ist hier anzumerken, dass die Untersuchung auf einem Querschnittsdesign basierte, wodurch die Interpretation kausaler Wirkbeziehungen im Strukturgleichungsmodell nur eingeschränkt möglich ist. So können die einzelnen Pfadkoeffizienten des PLS-Strukturmodells zwar signifikant und mit dem a priori postulierten Vorzeichen ausfallen, ihre kausale Wirkrichtung ist dadurch jedoch nicht als bestätigt zu werten.

Insgesamt zeigen die Ergebnisse dieser Arbeit, dass das Phänomen Informations- und Entscheidungsverhalten von Bewerbern vielschichtig und komplex ist (vgl. BoswelL et al., 2012). Allerdings war es nicht möglich, die oben angeführten ergänzenden Variablen in den jeweiligen Teilmodellen zu berücksichtigen, da ansonsten zum einen die Gefahr bestanden hätte, die Befragten in ihrem Informationsverarbeitungsprozess durch eine übermäßige Anzahl von zu fällenden Urteilen zu überfordern (vgl. BORTZ und DÖRING, 2010). Unter forschungsökonomischen Gesichtspunkten sollten hier ferner zunächst solche Prädiktoren einbezogen werden, die aufgrund von theoretischen Ansätzen oder empirischen Befunden über den höchsten angenommenen Erklärungsbeitrag verfügen. Die hier aufgestellten Modelle sollten durch nachfolgende Studien schrittweise in ihrer Vorhersagekraft verbessert werden, indem sie um weitere relevante Prädiktoren ergänzt werden. Dabei besteht eine zentrale Konsequenz für zukünftige Arbeiten darin, die bereits vorliegenden Erkenntnisse aus den beteiligten Forschungsdisziplinen noch stärker als bisher miteinander zu verzahnen, um zu einem interdisziplinären Forschungsansatz zu gelangen. Eine theoriegeleitete und differenzierte Integration der bereits existierenden marketingwissenschaftlichen und psychologischen Erkenntnisse und Modelle bietet eine fundierte Grundlage dafür, die noch offenen Forschungsfragen nach Ursachen, Zusammenhängen und Konsequenzen mithilfe neuer Sichtweisen und Lösungsstrategien zu beantworten.

Neben diesen theoretischen Aspekten sind auch methodische Begrenzungen anzuführen. Ein erster kritischer Punkt liegt in der nichtzufälligen Stichprobenauswahl mit dem damit einhergehenden Nachteil, den Stichprobenfehler statistisch nicht berechnen zu können. Dadurch ergibt sich auch grundsätzlich ein höheres Risiko, dass innerhalb der Stichprobenbildung bestimmte Teile in der Grundgesamtheit systematisch über- bzw. unterrepräsentiert sind, was wiederum zu einer tendenziell geringeren Repräsentativität der Stichprobe und dadurch geminderten externen Validität führen kann (vgl. KAYA und HIMME, 2009). Diese Einschränkung lässt sich 
zugleich insoweit relativieren, als dass die interne Validität bei den hier behandelten Teilmodellen im Vordergrund steht und die Generalisierbarkeit der hier erzielten Ergebnisse vorerst nachrangig zu behandeln war. Gleichwohl sollten weitere Studien versuchen, die Ergebnisse mit geeigneten zufälligen Auswahlverfahren zu replizieren. Eine zentrale Bedeutung sollte dann auch die Frage nach der externen Validität, d.h. nach der Verallgemeinerung der Befunde auf andere Zielgruppen, z.B. Studierende anderer Fachrichtungen, oder Branchen haben. Dementsprechend erscheint es sinnvoll, die Gültigkeit der hier aufgestellten Modelle anhand weiterer Zielgruppen zu validieren.

Ein weiteres Problem stellt die Single-Item-Messung der Berufsorientierungen dar, die ihrerseits dazu führt, dass keine interne Konsistenz für dieses Konstrukt berichtet werden kann und die Zuverlässigkeit der Messung möglicherweise geringer ist, als sie im Fall einer Multi-ItemMessung wäre. Um einen präziseren Einblick in die Einflussstärke der Berufsorientierungen in den beiden Teilmodellen zu erhalten, sollten in weiterführenden Untersuchungen ergänzende Multi-Item-Messungen der Berufsorientierungen entwickelt und eingesetzt werden. Alternative Einsatzmöglichkeiten lassen sich auch beim hier verwendeten Messanasatz der Branchenreputation feststellen. Während die Branchenreputation in dieser Untersuchung mittels eines reflektiven Messmodells erfasst wurde, erscheint es an dieser Stelle sinnvoll, zukünftig auch formative Messmodelle heranzuziehen, um die das Konstrukt verursachenden Faktoren näher bestimmen zu können (vgl. z.B. HELM, 2007; HAUTZINGER, 2009).

Mit der DCA liegt zweifelsohne ein sehr brauchbares Verfahren zur simultanen und damit relativen Bestimmung der Einflussstärke einzelner Arbeitsplatzmerkmale vor. Allerdings treten hier auch grundsätzliche Probleme dekompositioneller Verfahren zutage. So hängt die Erklärungskraft eines Discrete-Choice-Modells ganz entscheidend von der Auswahl der als relevant eingestuften Attribute und ihrer Ausprägungen ab (BACKHAUS et al., 2011). Daher wurde bei der experimentellen Umsetzung streng darauf geachtet, nur solche Arbeitsplatzmerkmale aufzunehmen, deren grundsätzliche Relevanz für die Arbeitgeberwahl in vorangegangenen Studien empirisch belegt werden konnte. Damit sollte zugleich sichergestellt werden, dass eine mögliche Nicht-Beachtung bestimmter Arbeitsplatzmerkmale methodisch und nicht inhaltlich zu interpretieren ist (AUSPURG et al., 2009). Ein weiteres Problem ist darin zu sehen, dass sich nicht alle Befragten den gleichen Wahlaufgaben gegenübersahen. Es ist daher nicht auszuschließen, dass die einzelnen Aufgaben-Decks einen Einfluss auf das Wahlverhalten der Befragten hatten, da sie jeweils einen spezifischen Entscheidungskontext schafften (KLEIN, 2006). Ferner ist zu beachten, dass das Vorgehen keine Verallgemeinerung über die bei den Arbeitsplatzmerkmalen verwendeten Ausprägungen hinaus erlaubt.

Darüber hinaus gilt grundsätzlich, dass das Ergebnis einer DCA umso aussagekräftiger ausfällt, je realitätsnäher die Wahlaufgaben gestaltet sind (SATTLER, 2006). Diesem Anspruch wurde 
bei dieser als extensiv charakterisierten Entscheidungssituation insofern Rechnung getragen, als dass die ausgewählten Arbeitsplatzmerkmale und ihre Ausprägungen auf Basis ausführlicher Recherchearbeiten in der Unternehmenspraxis sowie der Durchführung eines Pretests sorgfältig umgesetzt wurden. Gleichwohl waren die hier verwendeten Wahlaufgaben in einen hypothetischen Entscheidungskontext eingebettet, was die getroffenen Aussagen von der ihnen zugrunde liegenden Präsentationsform, wie beispielsweise von der Platzierung des Gütesiegels oder der tabellarischen Darstellung der Arbeitsplatzmerkmale, abhängig macht. In einer realen Entscheidungssituation finden die potenziellen Bewerber die Stellenangebote mit den entsprechenden Arbeitsplatzmerkmalen in der Regel nicht in einer solch systematisch aufbereiteten Form vor. Einige Arbeitsplatzmerkmale waren hierdurch möglicherweise stärker ins Bewusstsein der Befragten gerückt, als dies bei einem realen Stellenangebot der Fall sein würde. Eine möglichst realitätsnahe Gestaltung der Wahlsituation kann andererseits einer genaueren Ursachenanalyse und einer Verallgemeinerung der Ergebnisse entgegenstehen (ENNEKING, 2003). So hätte man bei der Verwendung weit verbreiteter visueller Elemente, wozu beispielsweise Unternehmenslogos, typische Produkte sowie die Darstellung und Beschreibung eigener Mitarbeiter zählen, nicht eindeutig ableiten können, ob die festgestellte Wirkung vollständig auf die Arbeitsplatzmerkmale oder auch teils auf anderweitige mit dem Arbeitgeberimage einhergehenden Assoziationen zurückzuführen ist (VON WALTER et al., 2009). Mithilfe der standardisierten Präsentationsform war es hierbei indes möglich, vom Einfluss anderer möglicher Faktoren zu abstrahieren, wodurch der Beitrag einzelner Arbeitsplatzmerkmale eindeutig zuzuordnen war. Es erscheint dennoch sinnvoll, in zukünftigen Studien den Einfluss weiterer, in der Praxis häufig verwendeter illustrativer Elemente als integralen Bestandteil eines Arbeitgeberimages näher zu untersuchen (vgl. MOSER et al, 1999). Ebenso wäre es interessant, mittels qualitativer Studien zu analysieren, welchen Aussagewert und welches Glaubwürdigkeitsniveau die beiden Gütesiegel bei den Befragten innehaben. Alternativ wäre es auch möglich, diese Aspekte mittels einer Einstellungsmessung zu erfassen. Erfolgt diese im Anschluss an die Wahlaufgaben, so würde die Kombination aus den beiden Messverfahren sowohl den Anforderungen eines realitätsnahen Entscheidungskontextes als auch einer differenzierten Ursachenanalyse nachkommen und die dabei resultierenden Informationen sinnvoll miteinander verknüpfen.

$\mathrm{Zu}$ wenig ist zudem bislang über die externe Validität der Ergebnisse aus DCA und die damit einhergehende Problematik der Verallgemeinerung hypothetischer Beurteilungsszenarien auf das tatsächliche Verhalten in realen Entscheidungssituationen potenzieller Bewerber bekannt (SATTLER, 2006). Erste Validierungsstudien weisen zugleich auf einen hohen Übereinstimmungsgrad zwischen hypothetischen und realen Entscheidungen hin, der sich sogar für tendenziell sozial erwünschte Entscheidungssituationen, wie beispielsweise den Kauf ökologischer Lebensmittel, nachweisen lässt (vgl. für einen Überblick: AUSPURG und LIEBE, 2011). Um die externe Validität der DC-Methodik weiterführend zu untermauern, sollten zukünftige Studien zur Arbeitgeberwahl versuchen, dem revealed-preference-Ansatz zu folgen und dabei konkrete 
Bewerbungen als objektive Verhaltensmaße zu erheben. Gleiches gilt auch für das Kausalmodell der Bewerbungsintention. So könnten mithilfe von Längsschnittstudien neben der vorgelagerten Intention auch das tatsächliche Bewerbungsverhalten erfasst werden.

\subsection{Implikationen für die Unternehmenspraxis}

Aus den wissenschaftlichen Erkenntnissen dieser Untersuchung lassen sich zugleich praxisrelevante Handlungsempfehlungen für mittelständische Unternehmen der Ernährungsindustrie ableiten, die sie gezielt zur Optimierung ihres bisherigen Personalmarketings einsetzen können. So lässt sich aus dem im ersten Teilmodell analysierten Informationsverhalten schlussfolgern, dass sich die einzelnen Maßnahmen des Personalmarketings darin unterscheiden, wie sie von der jeweiligen Zielgruppe wahrgenommen, genutzt und bewertet werden. Generell zeigt sich, dass Stellenanzeigen in Zeitschriften und Zeitungen, in Online-Stellenbörsen und auf der unternehmenseigenen Website sowie Praktika und Werkstudententätigkeiten potenzielle Bewerber in hohem Maße erreichen und von ihnen auch als nützlich erachtet werden. Anhand von soziodemographischen und persönlichkeitsspezifischen Merkmalen von potenziellen Bewerbern lassen sich zugleich unterschiedliche Zielgruppensegmente identifizieren, mit deren Hilfe die externe Bewerberansprache spezifischer und dadurch auch wirksamer gestaltet werden kann. Ist man also gezielt auf der Suche nach Bewerbern aus agrar-/ernährungswissenschaftlichen und lebensmitteltechnologischen Studiengängen, so bieten sich in diesem Zielgruppensegment insbesondere Unternehmensexkursionen als geeignete Kommunikationsmaßnahmen an. Masterstudierende erreicht man neben den oben genannten Rekrutierungswegen am wirksamsten über die Unterstützung von Praktika und Projektarbeiten, Kontakte zu Professoren relevanter Fachrichtungen sowie Fachvorträge von eigenen Unternehmensvertretern in Lehrveranstaltungen. Daneben werden aber auch hochschuleigene Datenbanken und Karrierenetzwerke, wie beispielsweise Xing, eher von dieser Zielgruppe frequentiert. Zur Bewerberansprache von Studierenden an Fachhochschulen sind indes Unternehmensexkursionen und Stellenausschreibungen auf der unternehmenseigenen Website besonders geeignet. Betrachtet man die beiden Maßnahmen aus dem Bereich sozialer Netzwerke, so zeigt sich interessanterweise ein den medial vermittelten Trends gegenläufiger Effekt: Sowohl Facebook als auch Xing nehmen im Vergleich zu allen übrigen Rekrutierungsquellen, zumindest derzeit, einen deutlich geringeren Stellenwert ein. Aus den einschlägigen Rekrutierungstrends geht jedoch hervor, dass die Bedeutung sozialer Netzwerke aufgrund ihres vergleichsweise kostengünstigen und weitreichenden Charakters zukünftig zunehmen wird, was wiederum zu einem erhöhten Handlungsbedarf auf der Unternehmensseite führt (WEITZEL et al., 2012).

Eine ergänzende Herangehensweise an das Problem zielgruppenspezifischer Auswahl von Rekrutierungsquellen besteht darin, neue Mitarbeiter kurz nach ihrem Organisationseintritt struk- 
turiert dazu zu befragen, wie sie auf das Unternehmen aufmerksam wurden und welche Medien bzw. Informationen sie dabei als besonders nützlich für ihre Bewerbungsentscheidung empfanden. Eine solche strukturierte Befragung würde zudem ein angemessenes Instrument des HR-Controllings darstellen, mit dessen Hilfe zukünftige Rekrutierungsaktivitäten effizienter geplant, gesteuert und kontrolliert werden könnten (vgl. TROST, 2012).

Bei der Gestaltung von Stellenangeboten ist es für Personalverantwortliche von großem Interesse, welchen Stellenwert potenzielle Bewerber bestimmten Arbeitsplatzmerkmalen beimessen. Aufgrund der Bedeutung dieser Fragestellung für die Gewinnung neuer Mitarbeiter sind zahlreiche Studien durchgeführt worden, deren praktischer Nutzen aus den oben dargestellten Gründen bislang jedoch nicht überzeugen kann. Die mittels der DCA ermittelten Bewerberpräferenzen stellen indes eine deutlich geeignetere Grundlage für eine vernünftige Leistungs- und Gehaltspolitik im Personalmarketing dar (SIMON et al., 1995). So können beispielsweise über die Ausgestaltung von Arbeitsverträgen variable leistungsabhängige und fixe Gehaltsanteile festgelegt werden, die aufgrund ihrer Entscheidungsrelevanz für potenzielle Bewerber die Akquisitionsfunktion des Personalmarketings unterstützen können (KIRCHGEORG und MÜLLER, 2011). Den mittelständischen Unternehmen aus der Ernährungsindustrie wird hierdurch ferner ermöglicht, potenziellen Bewerbern gezielt solche Eigenschaftsausprägungen anzubieten, die ihren eigenen Erwartungen entsprechen und somit auch ihre Bewerbungsbereitschaft steigern. Der Nutzen dieser Studie für die Unternehmenspraxis ist an dieser Stelle insbesondere darin zu sehen, dass die zugrunde liegenden Arbeitsplatzmerkmale einen deutlich höheren Konkretisierungsgrad und Anwendungsbezug aufweisen, als dies bei den bisherigen vergleichbaren Untersuchungen der Fall ist. Die betriebliche Umsetzung der hier erzielten Ergebnisse soll die Unternehmen allerdings nicht nur dazu verleiten, eine durchweg marktorientierte Sichtweise des Personalmarketings zu befolgen, die sich ausschließlich nach der Perspektive des externen Arbeitsmarktes, d.h. den Erwartungen potenzieller Bewerber, richtet (HUF, 2007). Bei diesem in der Unternehmenspraxis weit verbreiteten Vorgehen werden in einem dreistufigen Verfahren zunächst die zielgruppenspezifischen Erwartungen identifiziert und anschließend dem unternehmenseigenen Arbeitgeberimage gegenübergestellt. Im dritten Schritt wird hierauf basierend ein Soll-Ist-Vergleich erarbeitet, aus dem sich zugleich der Handlungsbedarf für das Personalmarketing ableitet. Neben den im Abschnitt 2.5.2 aufgezeigten methodischen Defiziten, geht eine solche Personalmarketingstrategie mit der Gefahr einher, eine Dissonanz zwischen dem extern kommunizierten und dem intern tatsächlich vorherrschenden Arbeitgeberimage zu schaffen, was wiederum in enttäuschten Bewerbererwartungen und einer erhöhten Fluktuationsrate münden kann (Rosenstiel und NeRDinger, 2000). Demgegenüber basiert der ressourcenorientierte Ansatz auf einer internen Analyse des Arbeitgeberimages. Bei diesem Vorgehen werden die Arbeitsplatzmerkmale im ersten Schritt durch die Mitarbeiter expliziert und danach mit der zukünftig geplanten Unternehmensentwicklung abgeglichen. Schließlich werden sie hinsichtlich ihrer Erfolgsrelevanz überprüft, die dann vorliegt, wenn die identifizierten Arbeitsplatz- 
merkmale wichtig, dauerhaft und unternehmensspezifisch sind und von der Zielgruppe auch entsprechend wahrgenommen werden (HUF, 2007). Eine erfolgversprechende Anwendungsmöglichkeit der hier ermittelten Bewerberpräferenzen besteht folglich darin, sie als fundierte Vergleichsbasis zum internen Arbeitgeberimage zu nutzen. Die Bewerberpräferenzen samt den dahinter liegenden Werten und Zielen können ferner systematisch dazu verwendet werden, bedarfsspezifische Personal- und Organisationsentwicklungsmaßnahmen abzuleiten und umzusetzen. Für die Kommunikationspolitik ergibt sich hieraus ferner die Aufgabe, potenzielle Bewerber schon während ihres Studiums transparent und verlässlich über das eigene Unternehmen zu informieren und ihnen dabei möglichst realistische Perspektiven zu vermitteln. Dabei sollten die mittelständischen Unternehmen vor allem auf solche Arbeitsplatzmerkmale setzen, die potenzielle Bewerber direkt beobachten können und die gleichzeitig als glaubwürdige Signale für die nicht beobachtbaren Arbeitsplatzmerkmale fungieren (SCHMIDTKE, 2001).

Derartig gestaltete Signale sind beispielsweise in den beiden hier untersuchen Gütesiegeln zu sehen. Die Ergebnisse verdeutlichen allerdings, dass die beiden Gütesiegel ,audit berufundfamilie" und „Top Job“ sowohl mit Chancen als auch mit Risiken verbunden sind. Imageförderliche Effekte von ,audit berufundfamilie“ sind demzufolge nur dann zu erwarten, wenn potenzielle Bewerber mit diesem Gütesiegel nicht vertraut sind. Im Falle seiner Bekanntheit, die derzeit noch sehr gering ausfällt, kann es sich indes auch ungünstig auf das zugrunde liegende Arbeitgeberimage auswirken. Eine ähnliche Wirkung konnte auch beim zweiten Gütesiegel „Top Job“ festgestellt werden, wobei es das Arbeitgeberimage nicht weiter beeinflusst, wenn es den Befragten bekannt ist. Mit diesem Ergebnis erfährt das wesentliche „Verkaufsargument“, die Gütesiegel würden grundsätzlich zu einer Imageverbesserung aufseiten der Unternehmen und zu einer höheren Bewerbungsbereitschaft aufseiten potenzieller Bewerber führen, erstmalig eine differenzierte ökonomische Beurteilung.

Auch weitere mögliche Risiken sollten in diesem Kontext bedacht werden (vgl. TAVAKKOLI, 2009; SCHERM und SÜSS, 2010; NAUNDORF und SPENGLER, 2012). So soll beispielsweise der Bekanntheitsgrad von Unternehmen aufgrund der mit der Gütesiegelvergabe einhergehenden Prämierungs- und Publikationsleistungen steigen. Allerdings konnte bislang nicht gezeigt werden, dass die hierbei genutzten Kommunikationsmaßnahmen potenzielle Bewerber auch tatsächlich erreichen. Aus der Unternehmensperspektive wäre es somit sinnvoll, die entsprechenden Kanäle hinsichtlich ihrer Wirksamkeit bei den relevanten Zielgruppen zu überprüfen. Darüber hinaus ließe sich der vorläufig positive Effekt einer höheren Anzahl von (Initiativ-)Bewerbungen, die beispielsweise aufgrund der vorhandenen Gütesiegel auf den Stellenanzeigen zustande kommen, auch insoweit ins Negative umkehren, als dass dadurch die Anzahl ungeeigneter Bewerbungen mit dem entsprechenden kostenverursachenden Bearbeitungsaufwand steigt. In diesem Kontext ist ferner zu bedenken, dass die Wettbewerbsteilnahme den Einsatz verschiedener Instrumente der Organisationsentwicklung, wie beispielsweise der Mitarbeiterbefragungen, voraussetzt, was wiederum in einem Mehraufwand für die Mitarbeiter mündet. 
Eine mit vergleichsweise lockeren Vergaberegeln verbundene Wettbewerbsteilnahme könnte darüber hinaus zu einem grundlegenden Interessensunterschied bis hin zu einem tiefgreifenden Zielkonflikt führen, da in diesem Fall neben dem eigenen Unternehmen auch solche genannt werden, die über eine deutlich schlechtere Unternehmensreputation bei potenziellen Bewerbern verfügen. Weiterhin könnte aus dem Einsatz von Gütesiegeln auch ein Konflikt mit der sog. Employee Value Proposition (EVP) des eigenen Unternehmens resultieren. Darunter wird das individuelle Arbeitgeberversprechen verstanden, das sich aus realistischen, zielgruppenspezifischen und wettbewerbsdifferenzierenden Arbeitsplatzmerkmalen zusammensetzt und analog zum Alleinstellungsmerkmal bzw. zur Unique Selling Proposition (USP) aus dem Marketing zu sehen ist (TROST, 2012). So könnte die unternehmensübergreifende Wirkung der Gütesiegel, die zugleich mit einem recht hohen Verbreitungsgrad einhergeht, leicht dazu führen, dass das Arbeitgeberversprechen des einzelnen Unternehmens nivelliert wird (vgl. SPILLER, 2001). Mittelständische Unternehmen, die bereits über ein gut entwickeltes Arbeitgeberimage verfügen oder dieses gerade aufbauen, werden somit weniger von dem Einsatz solcher Gütesiegel profitieren, als Unternehmen, die potenziellen Bewerbern kein klares Bild als Arbeitgeber vermitteln können. In diesem Kontext ist es für die Unternehmen ausgesprochen wichtig, die mit einer EVP einhergehenden Stärken und Besonderheiten durch konkrete Beweise zu untermauern (TROST, 2012). Um dies zu bewerkstelligen, sind weniger die i.d.R. standardisiert gehaltenen Informationen aus Arbeitgeberwettbewerben und Zertifizierungen erforderlich, sondern vielmehr ein qualitatives Vorgehen, das genügend Raum für Interpretationen und Kreativität zulässt.

Schließlich ist es auch denkbar, dass der Einsatz der Gütesiegel sich eher dann nachteilig auswirkt, wenn die darin transportierte Botschaft nicht zum tatsächlichen Arbeitgeberimage bzw. zur gelebten Unternehmenskultur passt und dadurch anderweitig durchgeführte Personalmarketingaktivitäten konterkariert. Mittelständische Unternehmen aus der Ernährungsindustrie, die Gütesiegel als Differenzierungsmerkmale nutzen wollen, sollten folglich die dargestellten Vor- und Nachteile systematisch abwägen, bevor sie dieses Instrument in ihrer Personalmarketingstrategie einsetzen.

In dieser Untersuchung ist schließlich der zentrale Stellenwert der Branchenreputation für die Gewinnung neuer Mitarbeiter deutlich herausgearbeitet worden. Mittelständische Unternehmen könnten in besonderem Maße davon profitieren, da potenzielle Bewerber die Branchenreputation als Vertrauenssignal nutzen, um ihre eigene Entscheidungsunsicherheit bei der Arbeitgeberwahl - zumindest ein Stück weit - zu reduzieren (vgl. EISENEGGER und KÜNSTLE, 2003). Als entscheidender Wettbewerbsvorteil fungiert sie insbesondere dann, wenn die Unternehmen branchenübergreifend um geeignete Mitarbeiter miteinander konkurrieren und eine positive Branchenreputation dabei zu einem wichtigen Differenzierungskriterium avanciert (BURMANN und SCHAEFER, 2005). Gleichzeitig stellt eine positive Branchenreputation die Basis dafür dar, dass potenzielle Bewerber ihre Unternehmenszugehörigkeit als prestigeträchtig für sich erachten und den damit einhergehenden Stolz bereits im Vorfeld ihres Organisationseintrittes 
antizipieren können. Analog dazu lässt sich über die Branchenreputation auch eine erhöhte Person-Organisation-Passung erreichen, wodurch die Selbstselektion seitens potenzieller Bewerber gesteigert werden kann, was wiederum zu kostensenkenden Effekten im Personalmarketing führt. Im Umkehrschluss bedeutet es jedoch auch, dass ein lädierter Branchenruf zu ungünstigen Konsequenzen in Form von Rechtfertigungen der eigenen Arbeitgeberwahl gegenüber dem sozialen Umfeld führen und dadurch in letzter Konsequenz auch eine negative Bewerbungsentscheidung begünstigen kann. Die negativen Folgen wiegen an dieser Stelle umso mehr, als dass die Reputation einer ganzen Branche nur sehr bedingt dem direkten Einfluss des unternehmenseigenen Personalmarketings unterliegt (HUF, 2007). Stattdessen ist eine deutlich gestiegene Bedeutung der Medien zur Beeinflussung der Reputation von Unternehmen und Branchen zu konstatieren, die dadurch einem erhöhten Reputationsrisiko ausgesetzt werden (EISENEGGER und IMHOF, 2009). Gleichzeitig lässt sich bei den potenziellen Bewerbern beobachten, dass sie nicht auf alle Rufaspekte im Einzelnen achten, sondern primär darauf, dass die Gesamtreputation intakt ist (HAUTZINGER, 2009). Es ist daher zu erwarten, dass insbesondere die „branchenfernen" potenziellen Bewerber im Falle einer in Bedrängnis geratenen Branchenreputation weniger Bereitschaft aufbringen, sich detailliert mit den zugrunde liegenden Ursachen bzw. einzelnen verantwortlichen Akteuren auseinanderzusetzen. Vor diesem Hintergrund bedarf es hier eines fortwährenden Reputationsmanagements, der aufgrund des damit einhergehenden enormen Aufwandes und seines weitreichenden Spektrums auf der Branchenebene, beispielsweise bei den einschlägigen Branchenverbänden, angesiedelt werden sollte. Wie ein solches Reputationsmanagement im Einzelnen zu gestalten ist, kann an dieser Stelle aus forschungsökonomischen Gründen nicht näher erläutert werden, hier sei auf die einschlägige Literatur zu diesem Forschungsgebiet verwiesen (z.B. NeUJAHR, 2012). Hinsichtlich der Gewinnung neuer Mitarbeiter ist gleichwohl die funktionale Rolle des Branchenwissens besonders hervorzuheben. Demzufolge wäre es sinnvoll, gezielt solche Kommunikationskanäle zu bedienen, die erheblich zum Wissenserwerb auf der Bewerberseite beitragen. Wie oben dargestellt, können persönlich-direkte Kommunikationsmaßnahmen, wie z.B. Unternehmensexkursionen oder Fachvorträge in Lehrveranstaltungen, dieses Vorhaben in hohem Maße unterstützen. Weiterführende Handlungsoptionen liegen im Bereich einer langfristigen Kandidatenbindung, die analog zu klassischen Vertriebsprozessen zu sehen ist und einen systematischen und nachhaltigen Umgang mit der relevanten Zielgruppe beinhaltet (TROST, 2012). Dabei ist es von zentraler Bedeutung, die einzelnen Aktivitäten auf die spezifischen Erwartungen und Präferenzen potenzieller Bewerber auszurichten. Neben der Einrichtung eines oder mehrerer „Talent-Pools“ wären in diesem Bereich gezielte Bindungsmaßnahmen, wozu beispielsweise regelmäßige Stipendienvergabe, Wochenendworkshops oder Mentoring-Programme zählen, denkbar. Die Bündelung solcher Aktivitäten auf der Branchenebene könnte allerdings auch ein gewisses Risiko für die beteiligten Unternehmen bergen, da es ihre individuellen Differenzierungsoptionen mindern könnte. Es wäre also stets abzuwägen, ob der Nutzenzuwachs aus einer gebündelten Branchenstrategie die teils verringerten unternehmenseigenen Differenzierungsoptionen überwiegt. 


\section{KAPITEL 8}

\section{Zusammenfassung}

Die gegenwärtige und auch zukünftige Situation auf dem Arbeitsmarkt stellt hohe Anforderungen an die Personalmarketingaktivitäten der Lebensmittelproduzenten. Gleichzeitig setzt sich in den Unternehmen zunehmend die Erkenntnis durch, dass vor allem diejenigen wirtschaftliche Erfolge erzielen können, denen es gelingt, besonders geeignete und leistungsfähige Mitarbeiter dauerhaft für sich zu gewinnen. Von dieser Herausforderung zeigen sich die überwiegend mittelständischen Unternehmen der Ernährungsindustrie in besonderem Maße betroffen, da sie häufig nicht über ein ausgeprägtes Image als Arbeitgeber verfügen und sich dadurch mit einer spürbar geminderten Arbeitgeberattraktivität für potenzielle Bewerber behaftet sehen.

Ausgehend von dieser Problematik besteht das Ziel dieser Arbeit darin, das Informations- und Entscheidungsverhalten potenzieller Bewerber in mittelständischen Unternehmen der Ernährungsindustrie systematisch zu untersuchen. Dazu wurden 671 Personen befragt, die kurz vor dem Abschluss ihres wirtschafts-, agrar-, ernährungswissenschaftlichen oder lebensmitteltechnologischen Studiums standen. Die zugrunde liegende Analyse erfolgt hierbei anhand von drei Teilmodellen. Im ersten Teilmodell steht das Informationsverhalten potenzieller Bewerber im Vordergrund, wobei hier für ausgewählte Maßnahmen des Personalmarketings untersucht wird, ob die Befragten diese zum einen kennen und nutzen, zum anderen inwieweit sie jene als nützlich für ihre eigene Arbeitgeberwahl einschätzen. Eine weiterführende Forschungsfrage besteht bei diesem Teilmodell darin, inwiefern die obigen Beurteilungskriterien mit den soziodemographischen und persönlichkeitsspezifischen Merkmalen der Befragten zusammenhängen, und ob letztere eine inkrementelle Validität gegenüber ersteren aufweisen. Es zeigen sich dabei positive Zusammenhänge mit den soziodemographischen Merkmalen, insbesondere mit den Prädiktoren Fachrichtung, Abschluss- sowie Hochschulart. Darüber hinaus zeigt sich, dass die persönlichkeitsspezifischen Merkmale in Form von Berufsorientierungen zusätzlich zu den soziodemographischen Prädiktoren Varianz in den Bewertungskriterien einiger Marketingmaßnahmen aufklären können. Allerdings fallen die Effektstärken der zugrunde liegenden Regressionsanalysen klein aus, sodass die Zusammenhänge insgesamt durchweg als gering einzustufen sind.

Das zweite Teilmodell beschäftigt sich indes mit der Forschungsfrage, welche Bewerberpräferenzen für die anfängliche Entscheidung, sich bei einem bestimmten Unternehmen zu bewer- 
ben, ausschlaggebend sind. Gemäß dem Modell der Person-Organisation-Passung wird hierbei postuliert, dass der Rekrutierungserfolg eines Unternehmens maßgeblich von der Übereinstimmung seiner Arbeitsplatzmerkmale mit den Präferenzen der potenziellen Bewerber abhängt. Dabei wird anhand von insgesamt fünf Arbeitsplatzmerkmalen mithilfe eines dekompositionellen Präferenzmessverfahrens, der Discrete-Choice-Analyse, untersucht, ob und inwieweit diese die Wahl von Stellenangeboten bei potenziellen Bewerbern beeinflussen. Zu den hier ausgewählten Attributen gehören auch zwei Gütesiegel, bei denen angenommen wird, dass sie aufgrund ihrer unsicherheitsreduzierenden Signalwirkung zu einer höheren Wahlwahrscheinlichkeit von Stellenangeboten führen. Ergänzend werden zudem die Berufsorientierungen potenzieller Bewerber in das Schätzmodell integriert, um differenzielle Effekte der obigen Arbeitsplatzmerkmale zu analysieren. Anders als bei der Verwendung direkter Befragungsmethoden zeigt sich, dass potenzielle Bewerber der Arbeitsplatzsicherheit eine große Bedeutung beimessen. Demgegenüber führt eine leistungsabhängige variable Vergütung zu einem geringeren Nutzen und folglich zu einer geringeren Wahlwahrscheinlichkeit von Stellenangeboten. Darüber hinaus kann für einzelne Arbeitsplatzmerkmale nachgewiesen werden, dass ihr Einfluss auf die Wahlwahrscheinlichkeit von den persönlichkeitsspezifischen Merkmalen potenzieller Bewerber abhängt. Die erwarteten positiven Effekte von Gütesiegeln lassen sich indes nur für den Fall bestätigen, dass sie potenziellen Bewerbern nicht bekannt sind. Andernfalls wirken sie sich nicht signifikant bzw. gar negativ auf die Wahlentscheidung der Befragten aus.

Zentrales Anliegen des dritten Teilmodells besteht darin, geeignete Determinanten der Bewerbungsabsicht aus den theoretischen Ansätzen der marketingwissenschaftlichen und psychologischen Forschung zu identifizieren und diese anhand eines Kausalmodells zu analysieren. Mit dem hier aufgestellten Kausalmodell ist es dabei gelungen, den Erklärungsbeitrag ausgewählter Prädiktoren auf die Bewerbungsabsicht in mittelständischen Unternehmen der Ernährungsindustrie empirisch zu bestätigen und weiter zu differenzieren. Den höchsten prädiktiven Beitrag zur Bewerbungsabsicht leistet dabei eindeutig die wahrgenommene Arbeitgeberattraktivität. Darüber hinaus zeigt sich, dass die Branchenreputation der Ernährungsindustrie einen bedeutsamen Stellenwert innerhalb des Kausalmodells einnimmt, wobei die hier postulierten Wirkbeziehungen partiell oder gar vollständig durch das Branchenwissen vermittelt werden. Die Ergebnisse dieser Arbeiten verdeutlichen zugleich, dass es sinnvoll ist, das Phänomen der Arbeitgeberwahl unter interdisziplinären Gesichtspunkten zu betrachten, und unterschiedliche vorhandene Erklärungsansätze gezielt dafür zu nutzen, die Theoriebildung auf diesem Gebiet voranzutreiben.

Neben ihrem wissenschaftlichen Beitrag bietet die Arbeit auch Personalverantwortlichen in mittelständischen Unternehmen der Ernährungsindustrie konkrete Anhaltspunkte, um künftige Personalmarketingaktivitäten zielgruppenspezifisch auszurichten. Dabei stellen die hier erzielten Erkenntnisse aus den einzelnen Teilmodellen eine wesentliche Grundlage für eine vernünftige Leistungs-, Gehalts- und Kommunikationspolitik dar. 


\section{A}

Aiman, L., T.N. BAueR und D.M. CABle (2001): Are You Attracted? Do You Intend To Pursue? A Recruiting Policy-Capturing Study. In: Journal of Business and Psychology 16 (2): 219237.

AIMAN-SMITH, L., S.E. SCULLEN und S.H. BARR (2002): Conducting Studies of Decision Making in Organizational Contexts: A Tutorial for Policy-Capturing and Other Regression-Based Techniques. In: Organizational Research Methods 5 (4): 388-414.

AJZEN, I. (1991): The Theory of Planned Behavior. In: Organizational Behavior and Human Decision Processes 50 (2): 179-211.

AJZEN, I. (2006): Constructing a Theory of Planned Behavior Questionnaire. In: www.people. umass.edu/aizen/pdf/tpb.measurement.pdf, abgerufen am 14.09.2011.

AJZEN, I. (2008): Consumer Attitudes and Behavior. In: Haugtvedt, C.P. (Hrsg.): Handbook of Consumer Psychology, New York u.a.: 525-548.

AKREMI, L. und N. BAUR (2011): Kreuztabellen und Kontingenzanalyse. In: Akremi, L. et al. (Hrsg.): Datenanalyse mit SPSS für Fortgeschrittene, Wiesbaden: 169-210.

AlBers, S. und L. HiLDEBRANDT (2006): Methodische Probleme bei der Erfolgsfaktorenforschung - Messfehler, formative versus reflektive Indikatoren und die Wahl des Strukturgleichungsmodells. In: Zeitschrift für betriebswirtschaftliche Forschung 58 (2): 2-33.

AlBersmeier, F. und A. SPILlER (2009): Das Ansehen der Fleischwirtschaft: Zur Bedeutung einer stufenübergreifenden Perspektive. In Böhm, J. et al. (Hrsg.): Die Ernährungswirtschaft im Scheinwerferlicht der Öffentlichkeit, Lohmar-Köln: 273-297.

Allen, D. G., R.V. MAHTO und R.F. OTONDO (2007): Web-Based Recruitment: Effects of Information, Organizational Brand, and Attitudes Toward a Web site on Applicant Attraction. In: Journal of Applied Psychology 92 (6): 1696-1708.

AndratschKe, N., S. REgiER und F. HubER (2009): Employer Branding als Erfolgsfaktor: Eine conjoint-analytische Untersuchung. Eul, Lohmar-Köln.

ARmitage, C.J. und J. Christian (2003): From Attitudes to Behaviour: Basic and Applied Research on the Theory of Planned Behaviour. In: Current Psychology 22 (3): 187-195. 
ARMitage, C.J. und M. CONNER (2001): Efficacy of the Theory of Planned Behaviour: A Meta-Analytic Review. In: British Journal of Social Psychology 40 (4): 471-499.

ARMSTRONG, M. (2012): Armstrong's Handbook of Human Resource Management Practice. Kogan Page, London.

ARNOLD, H.J. und D.C. FELDMAN (1981): Social Desirability Response Bias in Self-Report Choice Situations. In: Academy of Management Journal 24 (2): 249-259.

Arnold, J., J. LoAn-Clarke, C. CoOmbs, A. Wilkinson, J. PARK und D. Preston (2006): How Well Can the Theory of Planned Behavior Account for Occupational Intentions? In: Journal of Vocational Behavior 69 (3): 374-390.

Arthur, W., S.T. Bell, A.J. Villado und D. Doverspike (2006): The Use of Person-Organization Fit in Employment Decision Making: An Assessment of Its Criterion-Related Validity. In: Journal of Applied Psychology 91 (4): 786-801.

AuspurG, K., T. HinZ und S. LieBig (2009): Komplexität von Vignetten, Lerneffekte und Plausibilität im Faktoriellen Survey. In: Methoden, Daten, Analysen 3 (1): 59-96.

AuspurG, K. und U. LIEBE (2011): Choice-Experimente und die Messung von Handlungsentscheidungen in der Soziologie. In: Kölner Zeitschrift für Soziologie und Sozialpsychologie 63 (2): 301-314.

\section{B}

BACKHAUS, K., B. ERICHSON und R. WEIBER (2011): Fortgeschrittene multivariate Analysemethoden: Eine anwendungsorientierte Einführung. Springer, Berlin u.a.

BackHaus, K.B., B.A. StOnE und K. HeIner (2002): Exploring the Relationship Between Corporate Social Performance and Employer Attractiveness. In: Business \& Society 41 (3): 292-318.

BAMBERG, S. (1999): Umweltschonendes Verhalten - eine Frage der Moral oder der richtigen Anreize? In: Zeitschrift für Sozialpsychologie 30 (1): 57-76.

BAMBERG, S. (2002): Helfen Implementationsintentionen, die Lücke zwischen Absicht und Verhalten zu überwinden? In: Zeitschrift für Sozialpsychologie 33 (3): 143-155. 
BALDERJAHN, I. und P.M. WiedemanN (1999): Risikobewertungen im kognitiven Kontext. Arbeitspapier des Lehrstuhls für Betriebswirtschaftslehre mit dem Schwerpunkt Marketing der Universität Potsdam.

BARBER, A.E. (1998): Recruiting Employees: Individual and Organizational Perspectives. Sage, Thousand Oaks.

BArber, A.E. und M.V. Roehling (1993): Job Postings and the Decision to Interview: A Verbal Protocol Analysis. In: Journal of Applied Psychology 78 (5): 845-856.

BARNETT, M.L., J.M. JERMIER und B.A. LAFFERTY (2006): Corporate Reputation: The Definitional Landscape. In: Corporate Reputation Review 9 (1): 26-38.

BARON, R.M. und D.A. KENNY (1986): The Moderator-Mediator Variable Distinction in Social Psychological Research: Conceptual, Strategic, and Statistical Considerations. In: Journal of Personality and Social Psychology 51 (6): 1173-1182.

BAUER, T.N. und L. AimAn-SMith (1998): Green Career Choices: The Influence of Ecologic Stance on Recruiting. In: Journal of Business and Psychology 10 (4): 445-458.

BEACH, L.R. (1990): Image Theory: Decision Making in Personal and Organizational Contexts. Wiley, Chichester.

BEACH, L.R. und T.R. Mitchell (1987): Image Theory: Principles, Goals, and Plans in Decision Making. In: Acta Psychologica 66 (3): 201-220.

BeCKer, W., S. Fischer, M. StAFFel und P. Ulrich (2008): Individuelle Präferenzen und „Job Pursuit Intention“: Ergebnisbericht einer experimentellen Untersuchung. Bamberger Betriebswirtschaftliche Beiträge 151, Bamberg.

BECKER, W., M. STAFFEL und P. UlRICH (2011): Experimentelle Untersuchung des Bewerberverhaltens. Bamberger Betriebswirtschaftliche Beiträge 166, Bamberg.

BeckmanN, J. und H. HeckHAusen (2010): Motivation durch Erwartung und Anreiz. In: Heckhausen, J. und H. Heckhausen (Hrsg): Motivation und Handeln, Berlin u.a.: 105-143.

BERGKVIST, L. und J.R. Rossiter (2007): The Predictive Validity of Multiple-item Versus Singleitem Measures of the Same Constructs. In: Journal of Marketing Research 44 (2): 175-184. 
BLICKLE, G. (1999): Karriere, Freizeit, alternatives Engagement: Empirische Studien zum psychologischen Kontext von Berufsorientierungen. Hampp, München u.a.

BorG, I. und T. STAUfENBIEL (2007): Theorien und Methoden der Skalierung. Huber, Bern.

BORTZ, J. und N. DÖRING (2009): Forschungsmethoden und Evaluation für Human- und Sozialwissenschaftler. Springer, Heidelberg.

BORTZ, J. und C. SCHUSTER (2010): Statistik für Human- und Sozialwissenschaftler. Springer, Berlin u.a.

Bossow-Thies, S. und M. Clement (2009): Fuzzy Clustering mit Hilfe von Mixture Models. In: Albers, S. et al. (Hrsg.): Methodik der empirischen Forschung, Wiesbaden: 175-190.

Bossow-THIES, S. und G. PANTEN (2009): Analyse kausaler Wirkungszusammenhänge mit Hilfe von Partial Least Squares (PLS). In: Albers, S. et al. (Hrsg.): Methodik der empirischen Forschung, Wiesbaden: 365-380.

Bourhis, A. und R. MEKKAOUI (2010): Beyond Work-Family Balance: Are Family-Friendly Organizations More Attractive? In: Industrial Relations 65 (1): 98-117.

Boswell, W.R., M.V. RoEHLING, M.A. LEPINE und L.M. MOYNIHAN (2003): Individual JobChoice Decisions and The Impact of Job Attributes and Recruitment Practices: A Longitudinal Field Study. In: Human Resource Management 42 (1): 23-37.

Boswell W.R., R.D. Zimmerman und B.W. Swider (2012): Employee Job Search: Toward an Understanding of Search Context and Search Objectives. In: Journal of Management 38 (1): 129-163.

BÖTTGER, E. (2012): Employer Branding: Verhaltenstheoretische Analysen als Grundlage für die identitätsorientierte Führung von Arbeitgebermarken. Gabler, Wiesbaden.

BRocke, M. (2006): Präferenzmessung durch die Discrete Choice-Analyse: Effekte der Aufgabenkomplexität. Gabler, Wiesbaden.

BROwn, D. und R.K. CRACE (1996): Values in Life Role Choices and Outcomes: A Conceptual Model. Career Development Quarterly 44 (3): 211-223. 
BRunsteIn, J.C. und G. MAIER (1996). Persönliche Ziele: Ein Überblick zum Stand der Forschung. In: Psychologische Rundschau 47: 146-160.

BurmanN, C. und K. SCHAEFER (2005): Das Branchenimage als Determinante der Unternehmensmarkenprofilierung. Arbeitspapier des Lehrstuhls für innovatives Markenmanagement der Universität Bremen.

C

CABle, D.M. und M.E. Graham (2000): The Determinants of Job Seekers' Reputation Perceptions. In: Journal of Organizational Behaviour 21 (8): 929-947.

Cable, D.M und T.A. Judge (1994): Pay Preferences and Job Search Decisions: A PersonOrganization Fit Perspective. In: Personnel Psychology 47 (2): 317-348.

CABle, D.M und T.A. Judge (1996): Person-Organization Fit, Job Choice Decisions, and Organizational Entry. In: Organizational Behavior and Human Decision Processes 67 (3): 294311.'

CABle, D.M und T.A. Judge (1997): Applicant Personality, Organizational Culture, And Organization Attraction. In: Personnel Psychology 50 (2): 359-394.

CABLE, D.M. und D.B. TuRBAN (2001): Establishing the Dimensions, Sources, and Value of Job Seekers' Employer Knowledge During Recruitment. In: Ferris, G.R. (Hrsg.): Research in Personnel and Human Resource Management, New York: 115-163.

CABle, D.M. und D.B. Turban (2003): The Value of Organizational Reputation in the Recruitment Context: A Brand-Equity Perspective. In: Journal of Applied Social Psychology 33 (11): 2244-2266.

CARLESS, S.A. (2005): Person-Job Fit Versus Person-Organization Fit as Predictors of Organizational Attraction and Job Acceptance Intentions: A Longitudinal Study. In: Journal of Occupational and Organizational Psychology 78 (3): 411-429.

CARLESS, S.A. und A. IMBER (2007): Job and Organizational Characteristics: A Construct Evaluation of Applicant Perceptions. In: Educational and Psychological Measurement 67 (2): 328341. 
CARMELI, A. und A. FreUnD (2002): The Relationship between Work and Workplace Attitudes and Perceived External Prestige. In: Corporate Reputation Review 5 (1): 51-68.

CASPER, W.J. und L.C. BUfFARDI (2004): Work-Life Benefits and Job Pursuit Intentions: The Role of Anticipated Organizational Support. In: Journal of Vocational Behavior 65 (3): 391410 .

Chapman, D.S., K.L. Uggerslev, S.A. Carroll, K.A. Piasentin und D.A. Jones (2005): Applicant Attraction to Organizations and Job Choice: A Meta-Analytic Review of the Correlates of Recruiting Outcomes. In: Journal of Applied Psychology 90 (5): 928-944.

ChAPMAN, D. und J. WeBSTER (2006): Toward an Integrated Model of Applicant Reactions and Job Choice. In: The International Journal of Human Resource Management 17 (6): 10321057.

CHIN, W. (1998) The Partial Least Squares Approach to Structural Equation Modeling. In: Marcoulides, G. (Hrsg.): Modern Business Research Methods, New Jersey: 295-336.

Christophersen, T. und C. GRAPE (2009): Die Erfassung latenter Konstrukte mit Hilfe formativer und reflektiver Messmodelle. In: Albers, S. et al. (Hrsg.): Methodik der empirischen Forschung, Wiesbaden: 103-118.

ChrZAN, K. und B. ORME (2000): An Overview and Comparison of Design Strategies for Choice-Based Conjoint Analysis. Sawtooth Software Research Paper Series.

COBER, R.T., D.J. BROwN, L.M. KEEPIN und P.E. LEVY (2004): Recruitment on the Net: How Do Organizational Web Site Characteristics Influence Applicant Attraction? In: Journal of Management 30 (5): 623-646.

CoHEN, J. (1988): Statistical Power Analysis for The Behavioral Sciences. Erlbaum, Hillsdale u.a.

COLLINS, C.J. (2007): The Interactive Effects of Recruitment Practices and Product Awareness on Job Seekers' Employer Knowledge and Application Behaviors. In: Journal of Applied Psychology 92 (1): 180-190.

CONNElly, B.L., S.T. CERTO, R.D. IRELAND und REutzel, C. R. (2011): Signaling Theory: A Review and Assessment. In: Journal of Management 37 (1): 39-67. 
D

Davies, G., R. Chun, R.V. DA Silva und S. Roper (2004): A Corporate Character Scale to Assess Employee and Customer Views of Organization Reputation. In: Corporate Reputation Review 7 (2): 125-146.

De Cooman, R., S. De Gieter, R. Pepermans, S. Hermans, C. Du Bois, R. Caers und M. JEGERS (2009): Person-Organization Fit: Testing Socialization and Attraction-Selection-Attrition Hypotheses. In: Journal of Vocational Behavior 74 (1):102-107.

DineEn, B.R., S.R. ASH und R.A. NoE (2002): A Web of Applicant Attraction: Person-Organization Fit in the Context of Web-Based Recruitment. In: Journal of Applied Psychology 87 (4): 723-734.

DutTON, J.E., J.M. DuKERICH und C.V. HARQUAIL (1994): Organizational Images and Member Identification. In: Administrative Science Quarterly 39 (2): 239-263.

DOLL, J. und I. AJZEN (1992): Accessibility and Stability of Predictors in the Theory of Planned Behavior. In: Journal of Personality and Social Psychology 63(5): 754-765.

\section{$\mathbf{E}$}

EAgly, A.H. und S. Chaiken (1993): The Psychology of Attitudes. Wadsworth Thompson, Belmont.

ECKES, T. (1996): Typizitäts- und Spezifitätseffekte in der Einstellungs-Intentions-Relation. In: Zeitschrift für Sozialpsychologie 27 (1): 81-89.

ECKES, T. und B. SIX (1994): Fakten und Fiktionen in der Einstellungs-Verhaltens-Forschung: eine Meta-Analyse. In: Zeitschrift für Sozialpsychologie 25 (4): 253-271.

EDWARDS, M.R. (2010): An Integrative Review of Employer Branding and OB Theory. In: Personnel Review 39 (1): 5-23.

EgGert, A., G. FASSOTT und S. Helm (2005): Identifizierung und Quantifizierung mediierender und moderierender Effekte in komplexen Kausalstrukturen. In: Bliemel, F. et al. (Hrsg.): Handbuch Pfadmodellierung, Stuttgart: 101-116. 
EHRHART, K.H. und J.C. ZIEGERT (2005): Why Are Individuals Attracted to Organizations? In: Journal of Management 31 (6): 901-919.

EISENEGGER, M. und K. IMHOF (2009): Reputationsrisiken moderner Organisationen. In: Röttger, U. (Hrsg.): Theorien der Public Relations, Wiesbaden: 243-264.

EISENEGGER, M. und M. KÜNSTLE (2003): Reputation und Wirtschaft im Medienzeitalter. In: Die Volkswirtschaft 11: 58-62.

Elles, A., O. HARFFEn und M. IVAnOV (2012): Ist die Food-Branche fit für den künftigen Bewerbermarkt?, Bonn.

ENNEKING, U. (2003): Die Analyse von Lebensmittelpräferenzen mit Hilfe von DiscreteChoice-Modellen am Beispiel ökologisch produzierter Wurstwaren. In: Agrarwirtschaft 52 (5): 254-267.

EUROPÄISCHE KOMMISSION (2005): Die neue KMU-Definition: Benutzerhandbuch und Mustererklärung. In: ec.europa.eu/enterprise/policies/sme/files/sme_definition/sme_user_guide_ de.pdf, abgerufen am 7.11.2010.

\section{$\mathbf{F}$}

FASSOTT, G. (2005): Die PLS-Pfadmodellierung: Entwicklungsrichtungen, Möglichkeiten, Grenzen. In: Bliemel, F. et al. (Hrsg.): Handbuch Pfadmodellierung, Stuttgart: 19-30.

FASSOTT, G. und A. EGGERT (2005): Zur Verwendung formativer und reflektiver Indikatoren in Strukturgleichungsmodellen: Bestandsaufnahme und Anwendungsempfehlungen. In: Bliemel, F. et al. (Hrsg.): Handbuch Pfadmodellierung, Stuttgart: 31-48.

FAZIO, R.H. und M.P. ZANNA (1981): Direct Experience and Attitude-Behavior Consistency. In: Advances in Experimental Social Psychology 14: 161-202.

FORNELL, C. und D.F. LARCKER (1981): Evaluating Structural Equations Models with Unobservable Variables and Measurement Error. In: Journal of Marketing Research 18 (1): 39-50.

FRANKE, N. (2000): Personalmarketing gegenüber betriebswirtschaftlichem Führungsnachwuchs. In: Marketing ZFP 22 (1): 75-92. 
FRÖHLICH, W. (1987): Strategisches Personalmarketing. VDI-Verlag, Düsseldorf.

Fuchs, C. und A. Diamantopoulos (2010): Evaluating the Effectiveness of Brand Positioning Strategies from a Consumer Perspective. In: European Journal of Marketing 44 (11/12): 1763-1786.

G

Gatewood, R.D., M.A. Gowan und G.J. LAuTEnsChlager (1993): Corporate Image, Recruitment Image and Initial Job Choice Decision. In: Academy of Management Journal 36 (2): 414-427.

Gotsi, M. und A. M. WILSON (2001): Corporate Reputation: Seeking a Definition. In: Corporate Communication: An International Journal 6 (1), 24-30.

GÖTHLICH, S.E. (2009): Zum Umgang mit fehlenden Werten in großzahligen empirischen Erhebungen. In: Albers, S. et al. (Hrsg.): Methodik der empirischen Forschung, Wiesbaden: 119136.

GÖTZ, O. und K. LIEHR-GOBBERS (2004): Analyse von Strukturgleichungsmodellen mit Hilfe der Partial-Least-Squares(PLS)-Methode. In: Die Betriebswirtschaft 64 (6): 714-738.

GPRA E.V. (2011): GPRA-Vertrauensindex Q1/2011. In: www.gpra.de/news-media/vertrauensindex/newsdetails/?L=1 gpra-e-v\%2Fverbandsprofil\%2F\&tx_branews_pi1[item] $=52 \& \mathrm{cHash}=$ cc5a06abf668c0d907fef5bbddd97ed8, abgerufen am 20.04.2013.

GROBE, E. (2003): Corporate Attractiveness - eine Analyse der Wahrnehmung von Unternehmensmarken aus der Sicht von High Potentials. HHL-Arbeitspapier 50, Leipzig.

GROBE, E. (2008): Employer Branding als Forschungsgebiet der Marketingwissenschaft. In: Akademische Marketinggesellschaft e.V. und O. Klante (Hrsg.): Aktuelle Perspektiven des Marketingmanagements, Wiesbaden: 123-132.

GRUND, C. (2003): Arbeitsplatzpräferenzen und Besitzeffekte. In: Zeitschrift für Personalforschung 17 (1): 87-109. 
$\mathbf{H}$

HAir, J.F., M. SARSTEDT, C.M. Ringle und J.A. MENA (2012): An Assessment of the Use of Partial Least Squares Structural Equation Modeling in Marketing Research. In: Journal of the Academy of Marketing Science 40 (3): 414-433.

HAIR, J.F., C.M. RINGLE und M. SARSTEDT (2011): PLS-SEM: Indeed a Silver Bullet. In: Journal of Marketing Theory and Practice 19 (2): 139-151.

HAMBURGER ABENDBLATT (2012): Befristeter Job als verlängerte Probezeit. In: www.abendblatt.de/wirtschaft/karriere/article2177607/Befristeter-Job-als-verlaengerte-Probezeit.html, abgerufen am 07.02.2013.

HAMMANN, P. und B. ERICHSON (2000): Marktforschung. Gustav Fischer, Stuttgart.

HartmanN, A. und H. SATtler (2004): Wie robust sind Methoden zur Präferenzmessung? In: Zeitschrift für betriebswirtschaftliche Forschung 56 (1): 3-22.

HAUTZINGER, H. (2009): Der Ruf von Branchen: Eine empirische Untersuchung zur Messung, Wechselwirkung und Handlungsrelevanz des Branchenrufs. Gabler, Wiesbaden.

HeCKHAUSEN, J. und H. HeCKHAUSEN (2010): Motivation und Entwicklung. In: Heckhausen, J. und H. Heckhausen (Hrsg): Motivation und Handeln, Berlin u.a.: 427-488.

HELM, S. (2007): Unternehmensreputation und Stakeholder-Loyalität, Wiesbaden.

Henseler, J., C.M. Ringle und R. Sinkovics (2009): The Use of Partial Least Squares Path Modeling. In: Advances in International Marketing 20: 277-319.

Henseler, J. und M. SARSTEDT (2012): Goodness-Of-Fit Indices for Partial Least Squares Path Modeling. In: Computational Statistics 28 (2): 565-580.

HERRBACH, O. und K. MignONAC (2004): How Organisational Image Affects Employee Attitudes. In: Human Resource Management Journal 14 (4): 76-88.

Highhouse, S., F. Lievens und E.F. SinAR (2003): Measuring Attraction to Organizations. In: Educational and Psychological Measurement 63 (6): 986-1001. 
HighHouse, S., E.E. ThORnBuRY und I.S. LitTLE (2007): Social Identity Functions of Attraction to Organizations. In: Organizational Behavior and Human Decision Processes 103 (1):134146.

HildebrandT, L., H. KREIS und J. SCHWAlBACH (2008): Eine Analyse der Dimensionen des Fortune-Reputationsindex. Diskussionspapier 61 der Humboldt-Universität zu Berlin.

Himme, A. (2009): Conjoint-Analysen. In: Albers, S. et al. (Hrsg.): Methodik der empirischen Forschung, Wiesbaden: 283-298.

HofFMAN B.J. und D.J. WOEHR (2006): A Quantitative Review of the Relationship between Person-Organization Fit and Behavioral Outcomes. In: Journal of Vocational Behavior 68 (3): 389-399.

Holling, H. und T. MELLES (2004): Entscheidung und Nutzen. In: Schuler, H. (Hrsg.): Enzyklopädie der Psychologie. Organisationspsychologie I - Grundlagen und Personalpsychologie, Göttingen: 335-381.

Honeycutt, T.L. und B. Rosen (1997): Family Friendly Human Resource Policies, Salary Levels, and Salient Identity as Predictors of Organizational Attraction. Journal of Vocational Behavior 50 (2): 271-290.

HuBER, J. und K. ZwERINA (1996): The Importance of Utility Balance in Efficient Choice Designs. In: Journal of Marketing Research 33 (3): 307-317.

HUF, S. (2007): Arbeitgeberattraktivität und Arbeitgeber-Rankings: Wer ist der Attraktivste im Land? In: Personalführung 40 (12): 58-63.

HuNZIKER, P. (1973): Personalmarketing. Haupt, Bern.

INGLEHART, R. (1990): Kultureller Umbruch: Wertwandel in der westlichen Welt. Campus, Frankfurt am Main u.a.

INSTITUT FÜR MITTELSTANDFORSCHUNG BONN (2002): Definition und gesamtwirtschaftlicher Stellenwert des Mittelstandes. In: www.ifm-bonn.org/mittelstandsdefinition/definition-kmudes-ifm-bonn, abgerufen am 7.11.2010. 


\section{$\mathbf{J}$}

JARVis, C.B., S.B. MACKenZIE und P.M. PodsaKOFF (2003): A Critical Review of Construct Indicators and Measurement Model Misspecifications in Marketing and Consumer Research. In: Journal of Consumer Research 30 (2): 199-218.

Johnson, F.R., B.J. KANNinen, M. BIngham und S. ÖZDEMIR (2007): Experimental Design for Stated Choice Studies. In: KANNINEN, B.J. (Hrsg.): Valuing Environmental Amenities Using Stated Choice Studies, Dodrecht: 159-202.

JONAS, K. und J. DOLL (1996): Eine kritische Bewertung der Theorie überlegten Handelns und der Theorie des geplanten Verhaltens. In: Zeitschrift für Sozialpsychologie 27 (1): 18-31.

JuDGE, T.A. und R.D. BRETZ (1992): Effects of Work Values on Job Choice Decisions. In: Journal of Applied Psychology 77 (3): 261-271.

Judge, T.A. und D.M. CABLE (1997): Applicant Personality, Organizational Culture, and Organization Attraction. In: Personnel Psychology 50 (2): 359-394.

Jungermann, H., H.-R. Pfister und K. Fischer (2010): Die Psychologie der Entscheidung: Eine Einführung. Spektrum, Heidelberg.

$\mathbf{K}$

KAAS, K.P. und A. BUSCH (1996): Inspektions-, Erfahrungs- und Vertrauenseigenschaften von Produkten. In: Marketing Zeitschrift für Forschung und Praxis 18 (4): 243-252.

Kahneman, D. und A. TVersky (1979): Prospect Theory: An Analysis of Decision under Risk. In: Econometrica 47 (2): 263-291.

KANNING, U. P., J. SchmalBROCK und S. WILD (2009): Instrumente des Personalmarketings aus Sicht von Studierenden. In: Zeitschrift für Personalpsychologie 8 (3): 147-153.

KARREN, R.J. und M.W. BARRINGER (2002): A Review and Analysis of the Policy-Capturing Methodology in Organizational Research: Guidelines for Research and Practice. In: Organizational Research Methods 5 (4): 337-361.

KAY, R., O. SUPRINOVIC und A. WERNER (2010): Deckung des Fachkräftebedarfs in kleinen und mittleren Unternehmen. IfM-Materialien 200, Bonn. 
KAYA, M. und A. HimmE (2009): Möglichkeiten der Stichprobenbildung. In: Albers, S. et al. (Hrsg.): Methodik der empirischen Forschung, Wiesbaden: 79-88.

KLEIN, M. (2006): Der faktorielle Survey: Conjoint- und Vignettenanalyse. In: Behnke, J. et al. (2006): Methoden der Politikwissenschaft: Neuere qualitative und quantitative Analyseverfahren, Baden-Baden: 133-143.

KIRCHGEORG, M. und E. GÜNTHER (2006): Employer Brands zur Unternehmensprofilierung im Personalmarkt: Eine Analyse der Wahrnehmung von Unternehmensmarken auf der Grundlage einer deutschlandweiten Befragung von High Potentials. HHL-Arbeitspapier 74, Leipzig.

KirchgeorG, M. und A. LORBEER (2002): Anforderungen von High Potentials an Unternehmen: Eine Analyse auf der Grundlage einer bundesweiten Befragung von High Potentials und Personalentscheidern. HHL-Arbeitspapier 49, Leipzig.

KIRChGeORG, M. und J. MÜLler (2011): Personalmarketing als Schlüssel zur Gewinnung, Bindung und Wiedergewinnung von Mitarbeitern. In: Stock-Homburg, R. und B. Wolff (Hrsg.): Handbuch Strategisches Personalmanagement, Wiesbaden: 63-81.

KOLlAR, R. und M. STENGEL (1990): Sind Berufsorientierungen und organisationale Identifikation Chimären der Forschung? Zur Verhaltensrelevanz berufsbezogener Orientierungen und Einstellungen bei Führungskräften. In: Zeitschrift für Arbeits-und Organisationspsychologie 34 (2): $74-84$.

KoYs, D.J., T.J. KEAVENY und R.E. AlLEN (1989): Employment Demographics and Attitudes That Predict Preferences for Alternative Pay Increase Policies. In: Journal of Business and Psychology 4 (1): 27-47.

KraffT, M., O. GÖTZ und K. LIEHR-GobBERS (2005): Die Validierung von Strukturgleichungsmodellen mit Hilfe des Partial-Least-Squares (PLS)-Ansatz. In: Bliemel, F. et al. (Hrsg.): Handbuch Pfadmodellierung, Stuttgart: 71-86.

Kroeber-Riel, W., P. WeInBERG und A. GrÖPPEL-KleIN (2009): Konsumentenverhalten. Vahlen, München.

KNOX, S. und C. FREEMAN (2006): Measuring and Managing Employer Brand Image in the Service Industry. In: Journal of Marketing Management 22 (7/8):695-716. 
KOHLER, U. und F. KreUter (2008): Datenanalyse mit Stata: Allgemeine Konzepte der Datenanalyse und ihre praktische Anwendung. Oldenbourg, München.

KotLer, P. (1972): A Generic Concept of Marketing. In: Journal of Marketing 36 (2): 46-54.

KRISTOF, A.L. (1996): Person-Organization Fit: An Integrative Review of Its Conceptualizations, Measurement, and Implications. In: Personnel Psychology 24(1): 1-49.

Kristof-Brown, A.L., R.D. ZIMMERMAN und E.C. JOHNSON (2005): Consequences of Individuals' Fit at Work: A Meta-Analysis of Person-Job, Person-Organization, Person-Group, and Person-Supervisor Fit. In: Personnel Psychology 58 (2): 281-342.

KuHA, J. (2004): AIC and BIC: Comparisons of Assumptions and Performance. In: Sociological Methods \& Research 33 (2): 188-229.

KUHFELD, W.F. (o.J.): Orthogonal Arrays. In: support.sas.com/techsup/technote/ts723_Designs. txt, abgerufen am 14.09.2011.

$\mathbf{L}$

LANG VON-Wins, T., A. WitTMAnN, J. KASChUBE und L. VON ROSEnStiEL (1995): Wertgeleitete Stellenwahl und ihre Folgen für berufliche Einstellungen bei Fach- und Führungsnachwuchskräften. In: Zeitschrift für Arbeitswissenschaft 49 (4): 191-196.

LARSEN, D.A. und J.I. PHILLIPS (2002): Effect of Recruiter on Attraction to the Firm: Implications of the Elaboration Likelihood Model. In: Journal of Business and Psychology 16 (3): 347-364.

LAURENT, G. und J.-N. KAPFERER (1985): Measuring Consumer Involvement Profiles. In: Journal of Marketing Research 22 (1): 41-53.

LEMmink, J., A. SChuiJf und S. STREuKEnS (2003): The Role of Corporate Image and Company Employment Image in Explaining Application Intentions. Journal of Economic Psychology 24 (1): 1-15.

LEWANDOWSKI, A. und C. Liebig (2004): Determinanten der Arbeitgeberwahl und Relevanz des Personalimages für die Bewerbungsabsicht. In: Mannheimer Beiträge zur Wirtschafts- und Organisationspsychologie 19 (1): 15-28. 
Lievens, F., C. DeCAesteker, P. Coetsier und J. GeirnAert (2001): Organizational Attractiveness for Prospective Applicants: A Person-Organisation Fit Perspective. In: Applied Psychology: An International Review 50 (1): 30-51.

LIEVENS, F., G. VAN HOYE und F. ANSEEL (2007): Organizational Identity and Employer Image: Towards a Unifying Framework. In: British Journal of Management 18 (1): 45-59.

LIS, B. (2010): Corporate Social Responsibility: Eine empirische Analyse des Einflusses auf potentielle Arbeitnehmer. Kovač, Hamburg.

Louviere, J., D. Hensher und J.D. Swait (2000): Stated Choice Methods: Analysis and Application. Cambridge University Press, Cambridge.

M

Maier, G.W., G. RAPPEnSPERGER, L. VON Rosenstiel und I. ZWARG (1994): Berufliche Ziele und Werthaltungen des Führungsnachwuchses in den alten und neuen Bundesländern. In: Zeitschrift für Arbeits- und Organisationspsychologie 38 (1): 4-12.

MAIER, G.W., M. WASTIAN und L. VON ROSEnSTIEL (2009): Der differenzielle Einfluss der Berufsorientierungen auf Berufserfolg und Arbeitsmotivation: Ergebnisse einer Längsschnittstudie über 6 Jahre. In: Zeitschrift für Arbeits- und Organisationspsychologie 53 (3): 104-120.

MALMENDIER (2006): Ein Modell zur Arbeitsplatzwahl als Grundlage für ein RelationshipMarketing-Konzept im Bereich High-Potentials. Dissertation, Magdeburg.

MARCUS, B. (2011): Personalpsychologie. Verlag für Sozialwissenschaften, Wiesbaden.

MCCALL, J.J. (1965): The Economics of Information and Optimal Stopping Rules. In: Journal of Business 38 (3): 300-317.

MEFFERT, H., C. BuRMANN und M. KIRCHGEORG (2012): Marketing: Grundlagen marktorientierter Unternehmensführung. Gabler, Wiesbaden.

MOSER, K. (1992): Personalmarketing: Eine Einführung. Quintessenz, München.

Moser, K. (1995): Vergleich unterschiedlicher Wege zur Gewinnung neuer Mitarbeiter. In: Zeitschrift für Arbeits- und Organisationspsychologie 39 (3): 105-114. 
Moser, K., R. GrabarKIEWICZ und G. FinKeS (1999): Die Darstellung unternehmenskultureller Werte in visuellen Elementen von Stellenanzeigen. In: Zeitschrift Führung und Organisation 68 (1): 16-19.

Moser, K. und J. ZEMPEL (2004): Personalmarketing. In: Schuler, H. (Hrsg.): Enzyklopädie der Psychologie. Organisationspsychologie I - Grundlagen und Personalpsychologie, Göttingen: $389-438$.

Moser, K. und J. ZEMPEL (2006): Personalmarketing. In: Schuler, H. (Hrsg.): Lehrbuch der Personalpsychologie, Göttingen: 69-96.

$\mathbf{N}$

NAUNDORF, J. und T. SPEngLER (2012): Notwendige Bedingungen für die Aussagekraft von Employer-Award-Ergebnissen. In: Personal Quarterly 64: 28-33.

NERDINGER, F.W. (1994): Selbstselektion von potentiellen Führungsnachwuchskräften. In: von Rosenstiel et al. (Hrsg.): Fach- und Führungsnachwuchs finden und fördern, Stuttgart: 20-38.

NEUJAHR, E. (2012): Wer die Reputation hat, hat die Zukunft: Reputationsmanagement in Unternehmen, Frankfurt am Main u.a.

NIEDERAUER, C. (2009): Messung von Zahlungsbereitschaften bei industriellen Dienstleistungen. Gabler, Wiesbaden.

NiTZL, C. (2010): Eine anwenderorientierte Einführung in die Partial Least Square (PLS)-Methode. Arbeitspapier 21 des Instituts für Industrielles Management der Universität Hamburg.

NOLL, J. und M. WiNKLER (2004): Gütesiegel und Vertrauen im E-Commerce. In: Der Markt 43 (1): 23-32.

$\mathbf{O}$

ORDÓÑEZ, L.D., L. BENSON und L.R. BEACH (1999): Testing the Compatibility Test: How Instructions, Accountability, and Anticipated Regret Affect Prechoice Screening of Options. In: Organizational Behavior and Human Decision Processes 78 (1): 63-80. 
OSBORN, D.P. (1990): A Reexamination of the Organizational Choice Process. In: Journal of Vocational Behavior 36 (1): 45-60.

$\mathbf{P}$

PetTy, R.E. und J.T. CACIOPPO (1986): Communication and Persuasion: Central and Peripheral Routes to Attitude Change. Springer, New York u.a.

PetTy, R.E., D.T. Wegener und L.R. FABrigar (1997): Attitudes and Attitude Change. In: Annual Review of Psychology 48: 609-647.

PFISTER, H.-R. und U. KONERDING (1996): Erklärung und Vorhersage mit unsicheren Konsequenzen: Folgerungen aus der Entscheidungsforschung für die Einstellungsforschung. In: Zeitschrift für Sozialpsychologie 27 (1): 90-99.

POWER, D.J. und R.J. AldAG (1985): Soelberg's Job Search and Choice Model: A Clarification, Review, and Critique. In: Academy of Management Review 10 (1): 48-58.

$\mathbf{R}$

Reinartz, W., M. HAEnlein und J. Henseler (2009): An Empirical Comparison of the Efficacy of Covariance-Based and Variance-Based SEM. In: International Journal of Research in Marketing 26 (4): 332-344.

RESICK, C.J., B.B. BALTES und C.W. SHANTZ (2007): Person-Organization Fit and Work-Related Attitudes and Decisions: Examining Interactive Effects With Job Fit and Conscientiousness. In: Journal of Applied Psychology 92 (5): 1446-1455.

RHEINBERG, F. (2010): Intrinsische Motivation und Flow-Erleben. In: In: Heckhausen, J. und H. Heckhausen (Hrsg): Motivation und Handeln, Berlin u.a.: 365-388.

RILEY, J.G. (2001): Silver Signals: Twenty-Five Years of Screening and Signaling. In: Journal of Economic Literature 39 (2): 432-478.

RINGLE, C.M., S. WENDE und A. WILL (2005): Smart PLS 2.0 (beta). In: www.smartpls.de 
Roberson, Q.M., C.J. COLlins und S. OREG (2005): The Effects of Recruitment Message Specificity on Applicant Attraction to Organizations. In: Journal of Business and Psychology 19 (3): 319-339.

Rosenstiel, L. und F.W. Nerdinger (2000): Die Münchner Wertestudien: Bestandsaufnahme und (vorläufiges) Resümee. In: Psychologische Rundschau 51 (3): 146-157.

RYAN, R.M und E.L. DECI (2000): Intrinsic and Extrinsic Motivations: Classic Definitions and New Directions. In: Contemporary Educational Psychology 25 (1): 54-67.

RYNES, S.L. (1991): Recruitment, Job Choice, and Post-Hire Consequences: A Call for New Research Directions. In: Dunnette, M.D. und L.M. Howe (Hrsg): Handbook of Industrial and Organizational Psychology, Palo Alto: 399-444.

RYNES, S.L., D.T. SCHWAB und H.G. HENEMAN (1983): The Role of Pay and Market Pay Variability in Job Applicant Decisions. In: Organizational Behavior and Human Performance 31 (3): $353-364$.

$\mathbf{S}$

SAKS, A.M. und B.E. ASHFORTH (2002): Is Job Search Related to Employment Quality? It All Depends on the Fit. In: Journal of Applied Psychology 87 (4): 646-654.

SAKS, A.M., H.W. WIESNER und R.J. SUMMERS (1996): Effects of Job Previews and Compensation Policy on Applicant Attraction and Job Choice. In: Journal of Vocational Behavior 49 (1): 68-85.

SAMMER, K. und R. WÜSTENHAGEN (2006): Der Einfluss von Öko-Labelling auf das Konsumentenverhalten: Ein Discrete Choice Experiment zum Kauf von Glühbirnen. In: Pfriem, R. et al. (Hrsg.): Innovationen für eine nachhaltige Entwicklung, Wiesbaden: 469-486.

SATTLER, H. (2006): Methoden zur Messung von Präferenzen für Innovationen. In: Zeitschrift für betriebswirtschaftliche Forschung 54 (6): 154-176.

SCHAEFER, K. (2006): Branchenimages als Determinanten der Markenprofilierung. Deutscher Universitätsverlag, Wiesbaden.

SCHAMBERGER (2006): Differenziertes Hochschulmarketing für High Potentials. Books on Demand, Norderstedt. 
SCHERM, E. und S. SÜSS (2010): Personal-Awards: Legitimationsfassade, Mythos, Symbol? In: Zeitschrift Führung und Organisation 79 (4): 254-246.

SCHERM, E., S. SÜSS, und B. KRUSE (2010): Was ist gutes Personalmanagement? PersonalAwards: (K)eine neue Antwort. In: Personalführung 43 (9): 56-61.

SchneIDER, B. (1987): The People Make the Place. In: Personnel Psychology 40 (3): 437453.

SCHNEIDER, B., H.W. GoldSTEIN und D.B. SMITH (1995): The ASA Framework: An Update. In: Personnel Psychology 48 (4):747-779.

Schneider, B., D.B. Smith, J. FleEnOR und S. TAYlor (1998): Personality and Organizations: A Test of the Homogeneity Hypothesis. In: Journal of Applied Psychology 83, (3): 462470 .

SCHOLZ, C. (2000): Personalmanagement. Vahlen, München.

SCHMIDTKE, C. (2001): Der Mittelstand im Wettbewerb um Fachkräfte - Institutionenökonomisch fundiertes Personalmarketing als Lösungsansatz. Schriften zur Mittelstandsforschung 90, Wiesbaden.

SCHMIDTKE, C. (2002): Signaling im Personalmarketing: Eine theoretische und empirische Analyse des betrieblichen Rekrutierungserfolges. Hampp, München u.a.

Schreurs, B., C. Druart, K. Proost und K. De WitTe (2009): Symbolic Attributes and Organizational Attractiveness: The Moderating Effects of Applicant Personality. In: International Journal of Selection and Assessment 17 (1): 35-46.

SCHULER, H. und K. MOSER (1993): Entscheidung von Bewerbern. In: Moser, K. et al. (Hrsg.): Personalmarketing, Göttingen: 51-75.

SCHWAAB, M.O. (1991). Die Attraktivität deutscher Kreditinstitute bei Hochschulabsolventen: Eine empirische Untersuchung zum Personalmarketing. Schäffer, Stuttgart.

SCHWAB, D.P., S.L. RYNES und R.J. ALDAG (1987): Theories and Research on Job Choice and Choice. In: Rowland, K.M. und G.R. Ferris (Hrsg.): Research in Personnel and Human Resource Management, Greenwich: 129-166. 
Schwoerer, C. und B. Rosen (1989): Effects of Employment-At-Will Policies and Compensation Policies on Corporate Image and Job Pursuit Intentions. In: Journal of Applied Psychology 74 (4): 653-656.

SEnG, A. und J. STARYSTACH (2010): Studienergebnisse Der Befragung Zum Thema: Talentmanagement Im Mittelstand. Arbeitspapier der Hochschule für Oekonomie \& Management in Essen.

SimON, H., K. Wiltinger, K.-H. Sebastian und G. TACKE (1995): Effektives Personalmarketing. Gabler, Wiesbaden.

SIX, B. und T. ECKES (1996): Metaanalysen in der Einstellungs-Verhaltens-Forschung. In: Zeitschrift für Sozialpsychologie 27 (1): 7-17.

SoBEL, M.E. (1982): Asymptotic Confidence Intervals for Indirect Effects in Structural Equation Models. In: Leinhardt, S. (Hrsg.): Sociological Methodology, San-Francisco: 290-312.

SPENCE, M. (1973): Job Market Signaling. In: Quarterly Journal of Economics 87 (3): 355374.

SPENCE, M. (2002): Signaling in Retrospect and the Informational Structure of Markets. In: American Economic Review 92 (3): 434-459.

SPIEGEL ONLINE (2013): Hochschulabsolventen: Jeder Dritte hat befristete Stelle. In: www. spiegel.de/karriere/berufsstart/studie-jeder-dritte-hochschulabsolvent-hat-befristeten-arbeitsvertrag-a-879491.html, abgerufen am 07.02.2013.

SPILLER, A. (2001): Gütezeichen oder Markenartikel? In: Fleischwirtschaft 6: 47-50.

SPONHEUER, B. (2010): Employer Branding als Bestandteil einer ganzheitlichen Markenführung. Gabler, Wiesbaden.

STATISTISCHES BUNDESAMT (2009): Rückgang und Alterung der Bevölkerung im Erwerbsalter. In: www.destatis.de/DE/PresseService/Presse/Pressekonferenzen/2009/Bevoelkerung/Statement _Egeler_PDF.pdf?_blob=publicationFile, abgerufen am 28.10.2010.

STATISTISCHES BUNDESAMT (2010): Investitionserhebung der Unternehmen im Verarbeitenden Gewerbe mit 20 tätigen Personen und mehr. In: www.genesis.destatis.de/genesis/online, abgerufen am 28.10.2010. 
Steiner, P.M. und C. AtZMÜller (2006): Experimentelle Vignettendesigns in faktoriellen Surveys. In: Kölner Zeitschrift für Soziologie und Sozialpsychologie 58 (1): 117-146.

STENGEL, M. (1987): Identifikationsbereitschaft, Identifikation, Verbundenheit mit der Organisation oder ihren Zielen. In: Zeitschrift für Arbeits- und Organisationspsychologie 31 (4): 152-166.

StiglitZ, J. E. (2000): The Contributions of the Economics of Information to Twentieth Century Economics. In: Quarterly Journal of Economics 115(4): 1441-1478.

StOcK-HomburG, R. (2010): Personalmanagement. Gabler, Wiesbaden.

Stotz, W. und A. WeDEL (2009): Employer Branding: Mit Strategie zum bevorzugten Arbeitgeber. Oldenbourg, München.

SÜSS (1996): Externes Personalmarketing für Unternehmen mit geringer Branchenattraktivität. Hampp, München u.a.

\section{$\mathbf{T}$}

TAVAKKOLI, R. (2009): Arbeitgeberwettbewerbe und ihre Implikationen für Unternehmenskultur und Talent-Management. In: Wirtschaftspsychologie 11 (3): 32-41.

TEICHERT, T. (2000): Auswirkungen von Verfahrensalternativen bei der Erhebung von Präferenzurteilen. In: Marketing Zeitschrift für Forschung und Praxis 2: 145-159.

TEMME, J. (2009): Discrete-Choice-Modelle. In: Albers, S. et al. (Hrsg.): Methodik der empirischen Forschung, Wiesbaden: 299-314.

TEUfER, S. (1999): Die Bedeutung des Arbeitgeberimage bei der Arbeitgeberwahl: Theoretische Analyse und empirische Untersuchung bei High Potentials. Gabler, Wiesbaden.

TheuVsen, L. (2003): Erfolgsbedingungen leistungsorientierter Entgeltsysteme. In: Die Verwaltung 36 (4): 483-499.

Theuvsen, L., C. JAnZE und M. Heyder (2009): Agribusiness in Deutschland 2009: Selbstbewusst in volatilen Wachstumsmärkten, Hannover. 
THIERRY, H. (2001): The Reflection Theory on Compensation. In: Erez, M. et al. (Hrsg.): Work Motivation in the Context of a Globalizing Economy, Mahwah: 149-169.

TROMMSDORFF, V. (2009): Konsumentenverhalten. Kohlhammer, Stuttgart.

Trost, A. (2012): Talent Relationship Management: Personalgewinnung in Zeiten des Fachkräftemangels. Springer, Berlin ua.

TurBAN, D.B. (2001): Organizational Attractiveness as an Employer on College Campuses: An Examination of the Applicant Population. In: Journal of Vocational Behavior 58 (2): 293-312.

Turban, D.B. und D.W. GREENING (1996): Corporate Social Performance and Organizational Attractiveness to Prospective Employees. In: Academy of Managemenl Journal 40 (3): 658672.

Turban, D.B. und T.L. KeON (1993): Organizational Attractiveness: An Interactionist Perspective. In: Journal of Applied Psychology 78 (2): 184-193.

Turban, D.B., M.L. ForReT und C.L. HENDRICKSON (1998): Applicant Attraction to Firms: Influences of Organization Reputation, Job and Organizational Attributes, and Recruiter Behaviors. In: Journal of Vocational Behavior 52 (1): 24-44.

TUSCHKE, A. (2011): Führungskräftevergütung. In: Stock-Homburg, R. und B. Wolff (Hrsg.): Handbuch Strategisches Personalmanagement, Wiesbaden: 241-255.

$\mathbf{U}$

URBAN, D. (1993): Logit-Analyse. Statistische Verfahren zur Analyse von Modellen mitqualitativen Response-Variablen. Gustav Fischer, Stuttgart.

URBAN, D. und MAYERL, J. (2011): Regressionsanalyse: Theorie, Technik und Anwendung. Verlag für Sozialwissenschaften, Wiesbaden.

\section{$\mathbf{V}$}

VAN EERDE, W. und H. THIERRY (1996): Vroom‘s Expectancy Models and Work-Related Criteria: A Meta-Analysis. In: Journal of Applied Psychology 81 (5): 575-586. 
VON WALTER, B., S. HeNKEL und W. Heidig (2011): Mitarbeiterassoziationen als Treiber der Arbeitgeberattraktivität. In: Tomczak, T. (Hrsg.): Behavioral Branding: Wie Mitarbeiterverhalten die Marke stärkt, Wiesbaden: 295-315.

VÖlCKNER, F., H. SATTLER und T. TEICHERT (2008): Wahlbasierte Verfahren der ConjointAnalyse. In: Herrmann, A. et al. (Hrsg.): Handbuch Marktforschung: Methoden - Anwendungen - Praxisbeispiele, Wiesbaden: 689-711.

Vroom, V.H. (1966): Organizational Choice: A Study of Pre- and Post-Decision Making Processes. In: Organizational Behavior and Human Performance 1 (2): 212-225.

W

WEIBER, R. und J. ADLER (1995): Der Einsatz von Unsicherheitsreduktionsstrategien im Kaufprozess. In: Zeitschrift für betriebswirtschaftliche Forschung, Sonderheft 35: 61-77.

WeIBER, R. und MÜHLHAUS, D. (2010): Strukturgleichungsmodellierung: Eine anwendungsorientierte Einführung in die Kausalanalyse mit Hilfe von AMOS, SmartPLS und SPSS. Springer, Heidelberg u.a.

Weitzel, T., A. Eckhardt, A. Von Stetten, S. Laumer und C. Maier (2012): Recruiting Trends 2012. Arbeitspapier des Centre of Human Resources Information Systems der Universitäten Bamberg Frankfurt am Main.

WiedmanN, K.-P., C.J. Fombrun und C.B.M. VAN RiEL (2006): Ansatzpunkte zur Messung der Reputation von Unternehmen. In: Der Markt 45 (177): 98-109.

Williamson, I O., J.E. King JR., D. LEPAK und A. SARMA (2010): Firm Reputation, Recruitment Web Sites, and Attracting Applicants. In: Human Resource Management 49 (4): 669-687.

Wiseman, R. M., L.R. GomeZ-MEJIA und M. FugAte (2000): Rethinking Compensation Risk. In: Rynes, S.L. und B.A. Gerhart (Hrsg.): Compensation in Organizations: Current Research and Practice, San Francisco: 311-347.

WiLTINGER, K. (1997): Personalmarketing auf Basis von Conjoint-Analysen. In: Zeitschrift für Betriebswirtschaft 67 (3): 55-97.

WotTAwA, H. (2003): Lehrbuch Evaluation. Huber, Bern. 


\section{Anhang}




\section{0 \\ Hochschule Osnabrück \\ University of Applied Sciences}

Liebe Teilnehmerinnen, liebe Teilnehmer,

im Rahmen einer groß angelegten Studie untersucht das Fachgebiet Agrarmarketing der Hochschule Osnabrück die Fragestellung, auf welcher Grundlage sich potenzielle Bewerber für ihre zukünftigen Arbeitgeber entscheiden. Als mögliche Arbeitgeber werden in dieser Studie die Unternehmen der Ernährungsindustrie näher betrachtet. Zur Bearbeitung dieser Fragestellung kommt es uns auf Ihre Einschätzung an!

Schon jetzt vielen Dank für Ihre Unterstützung!

Die Bearbeitung des Fragebogens dauert ca. 15 Minuten. Es ist sehr wichtig, dass Sie ALLE Fragen beantworten. Dabei gibt es keine richtigen oder falschen Antworten, sondern es geht allein um Ihre persönlichen Einschätzungen und Erfahrungen mit Ihren potenziellen Arbeitgebern und dabei insbesondere um Unternehmen der Ernährungsindustrie.

Die Befragung erfolgt vollkommen anonym. Wir werden Sie am Anfang der Befragung nach einem Code fragen. Dieser Code ist nur Ihnen bekannt und dient alleine der Vermeidung von Doppelbefragungen. Eine Zuordnung Ihrer Antworten zu Ihrer Person ist unmöglich.

Noch ein letzter Hinweis: Wir verwenden in der Studie nur die männliche Form (z.B. Student, Professor) um die Lesbarkeit zu vereinfachen.

Bei Fragen zur Studie können Sie sich jederzeit gerne per E-Mail an mich wenden. Kontakt: m.abramovskij@hs-osnabrueck.de.

Nochmals vielen Dank für Ihre Teilnahme an unserer Arbeitgeberwahlstudie!

Ihre Marina Abramovskij

Dipl.-Psych. Marina Abramovskij

(Fachgebiet Agrarmarketing, Hochschule Osnabrück) 
Bevor es los geht, bitten wir Sie Ihren persönlichen Code anzugeben.

Der Code besteht aus 6 Stellen:

- Erster Buchstabe des Vornamens der Mutter als Großbuchstabe (z.B. Anna = A)

- Erster Buchstabe des Vornamens des Vaters als Großbuchstabe (z.B. Hans = H)

- $\quad$ Tag des eigenen Geburtstages (z.B. 8. Mai $1987=08$ )

- Geburtsjahr der Mutter (z.B. 23. August $1961=61$ )
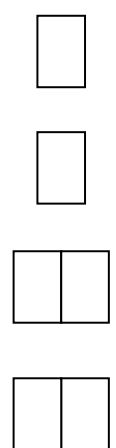

Jetzt benötigen wir noch einige Angaben zu Ihrer Person.

\section{Wie alt sind Sie?}

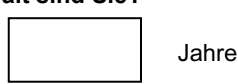

\section{Geschlecht}

$\bigcirc$ männlich

o weiblich

An welcher Hochschule studieren Sie?

(1) Universität

(2) Fachhochschule

Welches Studium absolvieren Sie derzeit?

(1) Bachelor

(2) Master

Um welche Art von Studium handelt es sich?

(1) Vollzeitstudium

(2) Fernstudium

(3) berufsbegleitendes Studium

(4) duales Studium

Welcher Fachrichtung ist Ihr Studiengang zuzuordnen?

Agrarwissenschaften

Ingenieurwissenschaften

O Naturwissenschaften

O Sozialwissenschaften

O Wirtschaftswissenschaften

Sonstiges 
In welchem Studiengang studieren Sie?

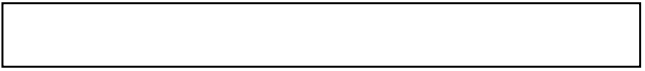

In welchem Fachsemester befinden Sie sich derzeit?

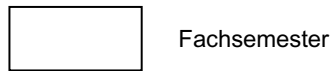

In wie vielen Semestern werden Sie voraussichtlich mit Ihrem Studium fertig sein?

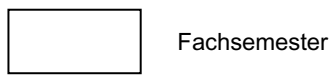

Verfügen Sie bereits über Berufserfahrung (z.B. durch Ausbildung, Praktika etc.)?

○ Ja

$\bigcirc \quad$ Nein

Wie viel Berufserfahrung konnten Sie bereits sammeln?

weniger als 6 Monate

- zwischen 6 Monaten und $1 \mathrm{Jahr}$

mehr als $1 \mathrm{Jahr}$

Wie viele Praktika (ausgenommen Schulpraktika) haben Sie bereits absolviert?

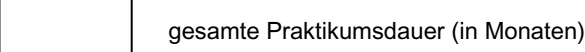

Befinden Sie sich derzeit aktiv auf Jobsuche?

○ Ja

Nein

Wenn ja, nach welcher Art von Beschäftigung sehen Sie sich derzeit um (Mehrfachnennung möglich)?
Nebenjob
○ Praktikum
- Abschlussarbeit
○ Festanstellung 
Zuerst würden wir gern mehr über Ihren persönlichen Bezug zur Ernährungsindustrie erfahren. Wie stark treffen die folgenden Aussagen zu?

\begin{tabular}{|c|c|c|c|c|c|c|}
\hline & & $\begin{array}{l}\text { trifft gar } \\
\text { nicht zu }\end{array}$ & $\begin{array}{l}\text { trifft wenig } \\
\mathrm{zu}\end{array}$ & $\begin{array}{l}\text { trifft mittel- } \\
\text { mäßig zu }\end{array}$ & $\begin{array}{l}\text { trifft über- } \\
\text { wiegend zu }\end{array}$ & $\begin{array}{l}\text { trifft voll- } \\
\text { ständig zu }\end{array}$ \\
\hline BRR2 & Die Ernährungsindustrie hat einen guten Ruf. & (1) & (2) & (3) & (4) & (5) \\
\hline BER3 & $\begin{array}{l}\text { Mein fachliches Wissen und meine Fähigkeiten kann } \\
\text { ich in der Ernährungsindustrie gut einbringen. }\end{array}$ & (1) & (2) & (3) & (4) & (5) \\
\hline BRW1 & Ich weiß ziemlich viel über die Ernährungsindustrie. & (1) & (2) & (3) & (4) & (5) \\
\hline BER1 & $\begin{array}{l}\text { Aufgrund meiner fachlichen Interessen und Schwer- } \\
\text { punkte kommt eine Tätigkeit in der Ernährungsindust- } \\
\text { rie für mich nicht in Frage. }\end{array}$ & (1) & (2) & (3) & (4) & (5) \\
\hline BRW3 & $\begin{array}{l}\text { Ich bin mit den Produkten der Ernährungsindustrie } \\
\text { vertraut. }\end{array}$ & (1) & (2) & (3) & (4) & (5) \\
\hline BRR1 & $\begin{array}{l}\text { Der Ruf der Ernährungsindustrie ist besser als der Ruf } \\
\text { anderer Branchen. }\end{array}$ & (1) & (2) & (3) & (4) & (5) \\
\hline BER2 & $\begin{array}{l}\text { Mein Studium ist darauf ausgerichtet, später in der } \\
\text { Ernährungsindustrie tätig zu sein. }\end{array}$ & (1) & (2) & (3) & (4) & (5) \\
\hline BRW2 & Ich bin mit der Ernährungsindustrie vertraut. & (1) & (2) & (3) & (4) & (5) \\
\hline BRR3 & $\begin{array}{l}\text { In der Öffentlichkeit genießt die Ernährungsindustrie } \\
\text { ein hohes Ansehen. }\end{array}$ & (1) & (2) & (3) & (4) & (5) \\
\hline
\end{tabular}

Nun würden wir gern erfahren, inwieweit Sie sich mit dem Thema „mein zukünftiger Arbeitgeber“ auseinandergesetzt haben und wie Sie Ihre aktuelle Situation auf dem Arbeitsmarkt einschätzen. Bitte geben Sie an, inwieweit folgende Aussagen auf Sie zutreffen.

\begin{tabular}{|c|c|c|c|c|c|c|}
\hline & & $\begin{array}{l}\text { trifft gar } \\
\text { nicht zu }\end{array}$ & $\begin{array}{l}\text { trifft wenig } \\
\text { zu }\end{array}$ & $\begin{array}{l}\text { trifft mittel- } \\
\text { mäßig zu }\end{array}$ & $\begin{array}{l}\text { trifft über- } \\
\text { wiegend zu }\end{array}$ & $\begin{array}{l}\text { trifft voll- } \\
\text { ständig zu }\end{array}$ \\
\hline INA4 & $\begin{array}{l}\text { Bisher habe ich mir zum Thema Arbeitsplatz keine } \\
\text { Gedanken gemacht. }\end{array}$ & (1) & (2) & (3) & (4) & (5) \\
\hline PIN3 & $\begin{array}{l}\text { Es sagt viel über einen Menschen aus, welchen Ar- } \\
\text { beitsplatz er auswählt. }\end{array}$ & (1) & (2) & (3) & (4) & (5) \\
\hline INA1 & $\begin{array}{l}\text { Ich habe mich ausführlich über die für mich in Frage } \\
\text { kommenden Arbeitgeber informiert. }\end{array}$ & (1) & (2) & (3) & (4) & (5) \\
\hline PIN2 & $\begin{array}{l}\text { Bei der Arbeitsplatzwahl kann man eigentlich keine } \\
\text { falschen Entscheidungen treffen. }\end{array}$ & (1) & (2) & (3) & (4) & (5) \\
\hline INA3 & $\begin{array}{l}\text { Ich habe mich ausführlich mit dem Thema „mein zu- } \\
\text { künftiger Arbeitgeber“ auseinandergesetzt. }\end{array}$ & (1) & (2) & (3) & (4) & (5) \\
\hline ARM1 & $\begin{array}{l}\text { Meine beruflichen Chancen auf dem Arbeitsmarkt } \\
\text { würde ich derzeit positiv einschätzen. }\end{array}$ & (1) & (2) & (3) & (4) & (5) \\
\hline PIN1 & $\begin{array}{l}\text { Die Wahl meines zukünftigen Arbeitsplatzes ist eine } \\
\text { sehr wichtige Entscheidung für mich. }\end{array}$ & (1) & (2) & (3) & (4) & (5) \\
\hline INA2 & $\begin{array}{l}\text { Ich habe bereits eine deutliche Vorstellung über poten- } \\
\text { zielle Arbeitgeber entwickelt. }\end{array}$ & (1) & (2) & (3) & (4) & (5) \\
\hline
\end{tabular}


Ferner würden wir gern wissen, worauf es Ihnen im Berufsleben besonders ankommt. Stellen Sie sich einmal vor, dass sich drei Studenten über ihre berufliche Zukunft unterhalten. Bitte geben Sie an, wie stark Sie folgenden Aussagen zustimmen.

\begin{tabular}{|c|c|c|c|c|c|c|c|c|}
\hline & & $\begin{array}{l}\text { stimme } \\
\text { überhaupt } \\
\text { nicht zu }\end{array}$ & $\begin{array}{l}\text { stimme } \\
\text { nicht zu }\end{array}$ & $\begin{array}{l}\text { stimme } \\
\text { eher } \\
\text { nicht zu }\end{array}$ & $\begin{array}{c}\text { stimme } \\
\text { teilweise zu }\end{array}$ & $\begin{array}{l}\text { stimme } \\
\text { eher zu }\end{array}$ & stimme zu & $\begin{array}{l}\text { stimme } \\
\text { voll und } \\
\text { ganz zu }\end{array}$ \\
\hline KAO1 & $\begin{array}{l}\text { Der erste sagt: „Ich möchte später } \\
\text { einmal in einer großen Organisation } \\
\text { in verantwortlicher Position tätig sein. } \\
\text { Dort habe ich die Möglichkeit, Einfluss } \\
\text { auf wichtige Geschehnisse zu neh- } \\
\text { men und werde außerdem gut be- } \\
\text { zahlt. Dafür bin ich gerne bereit, mehr } \\
\text { Zeit als vierzig Stunden in der Woche } \\
\text { zu investieren und auf Freizeit zu } \\
\text { verzichten." }\end{array}$ & (1) & (2) & (3) & (4) & (5) & (6) & (7) \\
\hline FRO1 & $\begin{array}{l}\text { Der zweite sagt: „Ich bin nicht so } \\
\text { ehrgeizig. Wenn ich eine sichere } \\
\text { Position mit geregelter Arbeitszeit } \\
\text { habe und mit netten Kollegen zu- } \\
\text { sammenarbeiten kann, bin ich zufrie- } \\
\text { den. Die mir wichtigen Dinge liegen } \\
\text { nicht in der Arbeitszeit, sondern in der } \\
\text { Freizeit - und dafür brauche ich auch } \\
\text { nicht sehr viel Geld.“" }\end{array}$ & (1) & (2) & (3) & (4) & (5) & (6) & (7) \\
\hline ALE1 & $\begin{array}{l}\text { Der dritte sagt: „Ich bin durchaus } \\
\text { bereit, viel Arbeitskraft zu investieren, } \\
\text { aber nicht in einer großen Organisati- } \\
\text { on der Wirtschaft oder Verwaltung, die } \\
\text { nur auf Profit ausgerichtet ist. Ich } \\
\text { möchte einmal in einer anderen Ar- } \\
\text { beitswelt tätig sein, in der ich mich } \\
\text { selbst verwirklichen und einen gesell- } \\
\text { schaftliche Beitrag leisten kann. Dafür } \\
\text { bin ich bereit, auf hohe Bezahlung } \\
\text { oder auf Statussymbole zu verzich- } \\
\text { ten.“ }\end{array}$ & (1) & (2) & (3) & (4) & (5) & (6) & (7) \\
\hline
\end{tabular}

Als nächstes möchten wir Sie bitten, die nachstehenden fiktiven Stellenangebote vor dem Hintergrund der folgenden Situation zu beurteilen:

Sie befinden sich am Ende Ihres Studiums und möchten sich demnächst um eine Stelle bewerben. Nun liegt Ihnen das Stellenangebot eines mittelständischen Unternehmens aus der Ernährungsindustrie vor. Der potenzielle Arbeitgeber erfüllt alle aus Ihrer Sicht relevanten fachlichen und regionalen Kriterien wie z.B. Art der Tätigkeit und Stand- bzw. Einsatzort - diese sollen hier nicht näher erläutert werden. Andere Kriterien werden im Stellenangebot dagegen explizit aufgeführt. Die folgenden Angebote unterscheiden sich hinsichtlich der Kriterien

- Bezahlung

- Arbeitsplatzsicherheit

- Vereinbarkeit von Beruf und Familie/Privatleben sowie

- Aufstiegs- und Entwicklungschancen

- Zudem wurden einige Unternehmen durch unabhängige Anbieter mit einem Gütesiegel ausgezeichnet.

Bitte geben Sie für jede der folgenden Entscheidungssituationen an, welches Stellenangebot Sie am meisten anspricht (nur ein Stellenangebot pro Entscheidungssituation ankreuzen). 
Ein mittelständisches Unternehmen aus der Ernährungsindustrie bietet Ihnen:

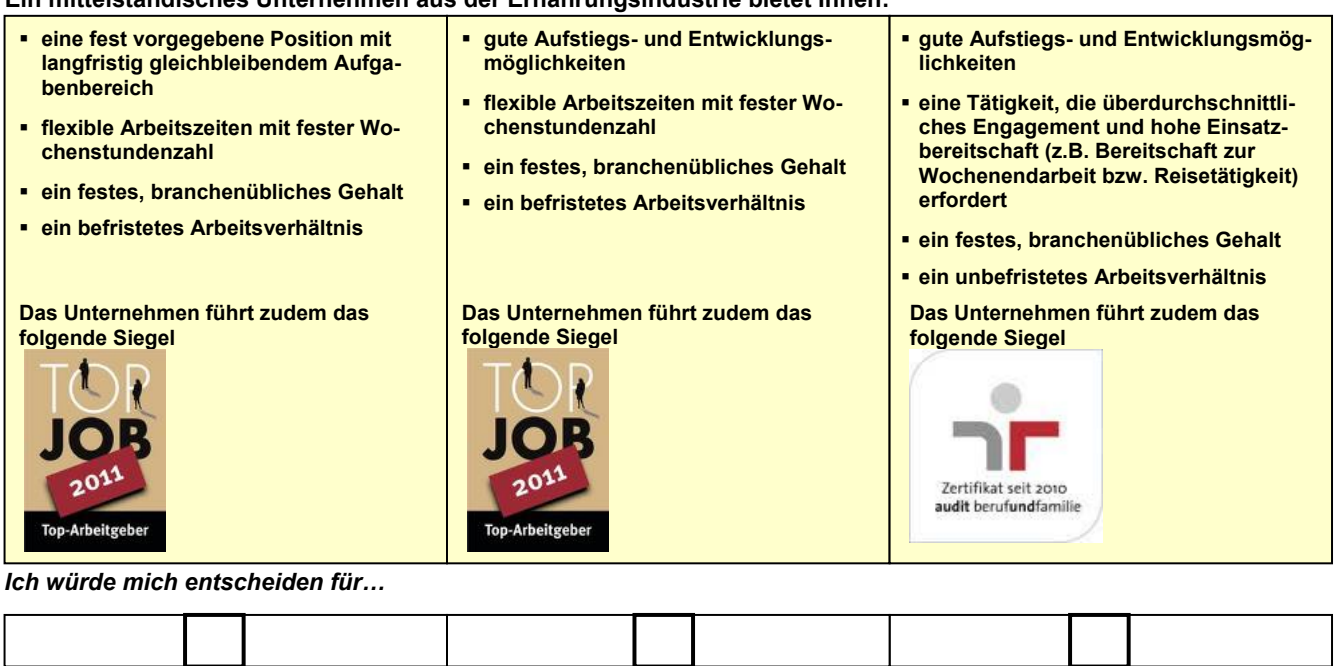

Würden Sie auch dann dieses Angebot wählen, wenn Sie nicht zwingend aus diesen drei Angeboten eine Auswahl treffen müssten?

(1) Ja, ich würde bei meiner Wahl bleiben.

(2) Nein, ich würde keines der Angebote wählen.

Ein mittelständisches Unternehmen aus der Ernährungsindustrie bietet Ihnen:

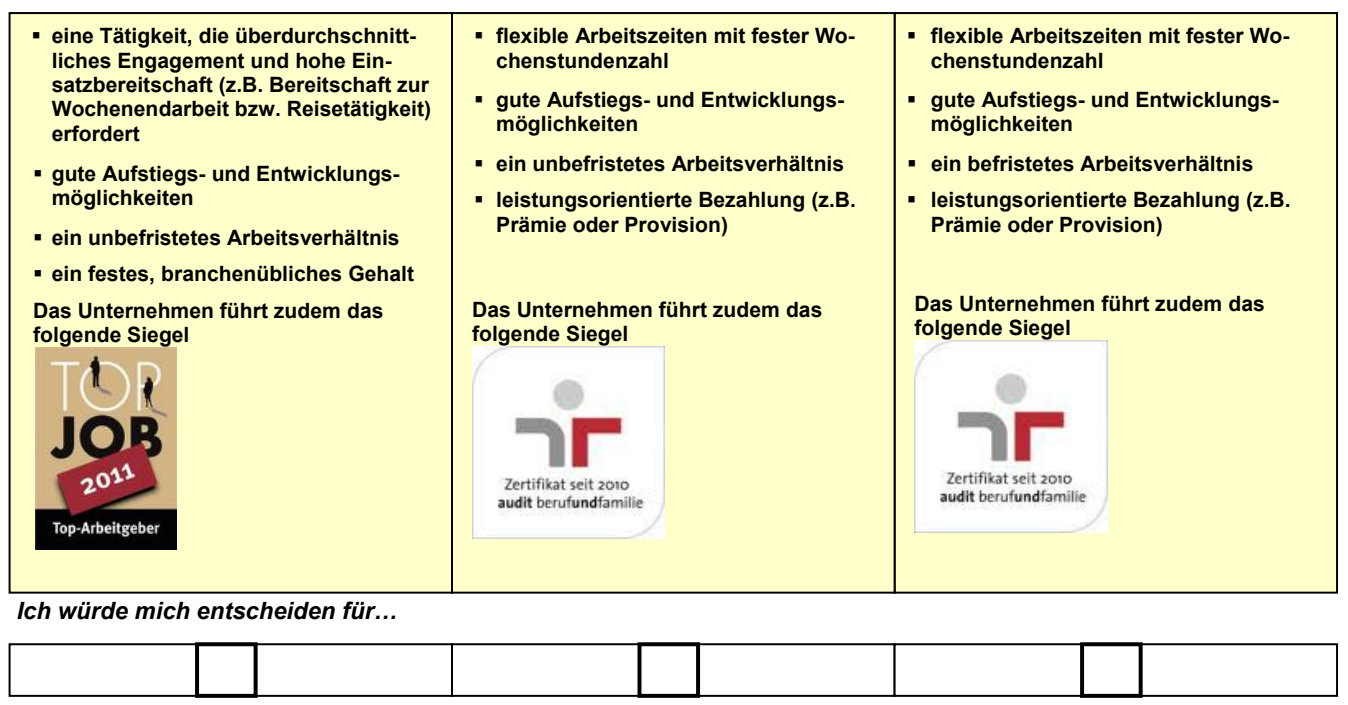

Würden Sie auch dann dieses Angebot wählen, wenn Sie nicht zwingend aus diesen drei Angeboten eine Auswahl treffen müssten?

(1) Ja, ich würde bei meiner Wahl bleiben.

(2) Nein, ich würde keines der Angebote wählen. 
Ein mittelständisches Unternehmen aus der Ernährungsindustrie bietet Ihnen:

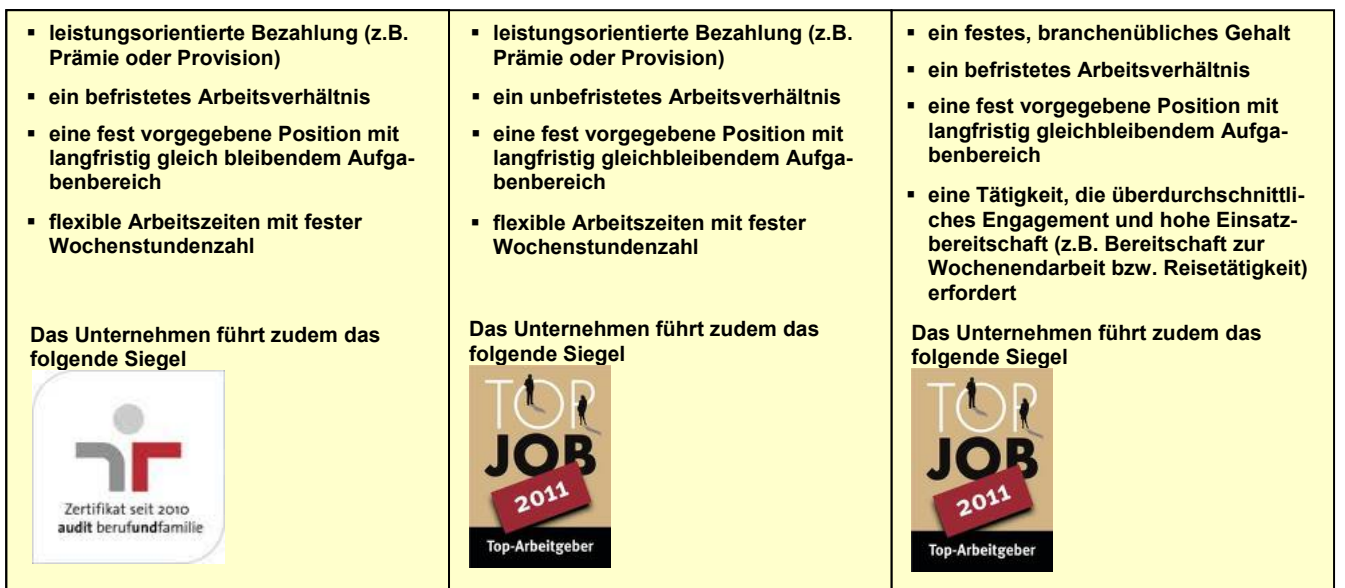

Ich würde mich entscheiden für...

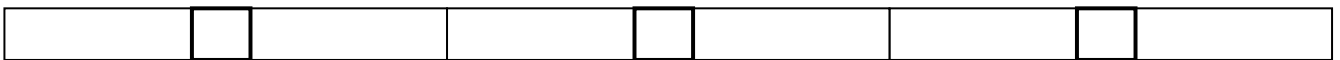

Würden Sie auch dann dieses Angebot wählen, wenn Sie nicht zwingend aus diesen drei Angeboten eine Auswahl treffen müssten??

(1) Ja, ich würde bei meiner Wahl bleiben.

(2) Nein, ich würde keines der Angebote wählen.

Wie schwer ist es Ihnen gefallen, sich zwischen den einzelnen Stellenangeboten zu entscheiden?

(1) Es ist mir schwer gefallen und ich musste länger nachdenken, bevor ich angekreuzt habe.

(2) Es ist mir leicht gefallen und ich konnte spontan ankreuzen.

Ist Ihnen das Gütesiegel audit berufundfamilie vorher bekannt gewesen (z.B. aus Karriereseiten im Internet, Imagebroschüren oder Stellenanzeigen von Unternehmen)?

(1) Ja, es ist mir vorher bekannt gewesen.

(2) Nein, es ist mir vorher nicht bekannt gewesen.

Ist Ihnen das Gütesiegel TOP JOB vorher bekannt gewesen (z.B. aus Karriereseiten im Internet, Imagebroschüren oder Stellenanzeigen von Unternehmen)?

(1) Ja, es ist mir vorher bekannt gewesen

(2) Nein, es ist mir vorher nicht bekannt gewesen.

\begin{tabular}{|c|c|c|c|c|c|}
\hline & $\begin{array}{c}\text { völlig } \\
\text { unwichtig }\end{array}$ & $\begin{array}{l}\text { eher un- } \\
\text { wichtig }\end{array}$ & teil teils & $\begin{array}{c}\text { eher } \\
\text { wichtig }\end{array}$ & $\begin{array}{l}\text { sehr wich- } \\
\text { tig }\end{array}$ \\
\hline $\begin{array}{l}\text { Wie wichtig ist es insgesamt für Sie, dass Ihr potenzieller Arbeit- } \\
\text { geber eine Auszeichnung in Form eines Gütesiegels (wie z.B. } \\
\text { audit berufundfamilie oder TOP JOB) führt? }\end{array}$ & (1) & (2) & (3) & (4) & (5) \\
\hline
\end{tabular}


Nun würden wir Sie gern bitten, das folgende Stellenangebot etwas detaillierter zu beurteilen.

Auch hier gilt: Der potenzielle Arbeitgeber erfüllt alle aus Ihrer Sicht relevanten fachlichen und regionalen Kriterien wie z.B. Art der Tätigkeit und Stand- bzw. Einsatzort.

\section{Tradition trifft Zukunft}

Die Food $\mathrm{GmbH}$ ist ein mittelständisches Unternehmen der produzierenden Lebensmittelwirtschaft mit Sitz an einem für Sie attraktiven Standort. Seit 50 Jahren steht der Name für vielseitige Produkte und höchste Ansprüche an deren Qualität. Mit rund 200 Mitarbeiterinnen und Mitarbeitern ist die Food $\mathrm{GmbH}$ führend in ihrem Segment und setzt Maßstäbe in den

Bereichen Herstellung, Vertrieb und Innovationen.

Zum nächstmöglichen Zeitpunkt sucht das Unternehmen eine fachlich wie menschlich überzeugende

Persönlichkeit

für eine Aufgabe, die sowohl Ihrer fachlichen Qualifikation als auch Ihren Interessen entspricht.

Ihr Profil:

Sie haben vorzugsweise ein wirtschafts-, agrar- oder ernährungswissenschaftliches Studium erfolgreich absolviert. Sie sind offen, engagiert, beherrschen MS-Office und haben idealerweise erste Berufserfahrungen gesammelt.

Was Sie bei der Food GmbH erwartet:

- gute Aufstiegs- und Entwicklungsmöglichkeiten

- flexible Arbeitszeiten mit fester Wochenstundenzahl

- ein festes, branchenübliches Gehalt

- ein unbefristetes Arbeitsverhältnis

Die Food $\mathrm{GmbH}$ führt zudem ein Gütesiegel, das ihr durch einen unabhängigen Anbieter verliehen wurde:

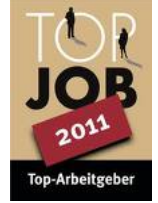

Wenn Sie Interesse haben, dann bewerben Sie sich bei der Food $\mathrm{GmbH}$ 
Ergänzen Sie bitte die folgenden Aussagen durch Ankreuzen der passenden Antwort (1 bis 5).

\begin{tabular}{|c|c|c|c|c|c|c|}
\hline & & $\begin{array}{c}\text { sehr } \\
\text { schlecht }\end{array}$ & & & & $\begin{array}{c}\text { sehr } \\
\text { gut }\end{array}$ \\
\hline \multirow[t]{2}{*}{ ATT1 } & $\begin{array}{l}\text { Wenn ich daran denke, bei diesem Unternehmen zu } \\
\text { arbeiten, ist mein Gefühl... }\end{array}$ & (1) & (2) & (3) & (4) & (5) \\
\hline & & $\begin{array}{l}\text { überhaupt } \\
\text { nicht } \\
\text { attraktiv }\end{array}$ & & & & $\begin{array}{l}\text { sehr } \\
\text { attraktiv }\end{array}$ \\
\hline \multirow[t]{2}{*}{ ATT2 } & $\begin{array}{l}\text { Als Arbeitgeber wäre dieses Unternehmen für mich } \\
\text { persönlich... }\end{array}$ & (1) & (2) & (3) & (4) & (5) \\
\hline & & $\begin{array}{l}\text { überhaupt } \\
\text { nicht } \\
\text { anspre- } \\
\text { chend }\end{array}$ & & & & $\begin{array}{l}\text { sehr an- } \\
\text { sprechend }\end{array}$ \\
\hline \multirow[t]{2}{*}{ ATT3 } & $\begin{array}{l}\text { Eine Beschäftigung bei diesem Unternehmen wäre für } \\
\text { mich... }\end{array}$ & (1) & (2) & (3) & (4) & (5) \\
\hline & & $\begin{array}{c}\text { sehr } \\
\text { unange- } \\
\text { nehmen }\end{array}$ & & & & $\begin{array}{c}\text { sehr ange- } \\
\text { nehm }\end{array}$ \\
\hline ATT4 & $\begin{array}{l}\text { Die Vorstellung, bei diesem Unternehmen zu arbeiten, } \\
\text { empfinde ich persönlich als... }\end{array}$ & (1) & (2) & (3) & (4) & (5) \\
\hline
\end{tabular}

Wie stark treffen die folgenden Aussagen auf das oben dargestellte Stellenangebot zu?

\begin{tabular}{|c|c|c|c|c|c|c|}
\hline & & $\begin{array}{l}\text { stimme } \\
\text { überhaupt } \\
\text { nicht zu }\end{array}$ & $\begin{array}{l}\text { stimme } \\
\text { eher nicht } \\
\text { zu }\end{array}$ & weder noch & $\begin{array}{l}\text { stimme } \\
\text { eher zu }\end{array}$ & $\begin{array}{l}\text { stimme voll } \\
\text { und ganz } \\
\text { zu }\end{array}$ \\
\hline POR1 & $\begin{array}{l}\text { Mit meiner Persönlichkeit passe ich gut in das Unter- } \\
\text { nehmen. }\end{array}$ & (1) & (2) & (3) & (4) & (5) \\
\hline INT1 & $\begin{array}{l}\text { Auf dieses Stellenangebot würde ich mich bewerben, } \\
\text { wenn ich einen Job suchen würde. }\end{array}$ & (1) & (2) & (3) & (4) & (5) \\
\hline POR2 & $\begin{array}{l}\text { Das Unternehmen stimmt mit meinen persönlichen } \\
\text { Erwartungen überein. }\end{array}$ & (1) & (2) & (3) & (4) & (5) \\
\hline INT2 & $\begin{array}{l}\text { Wenn ich mit dem Studium fertig wäre, würde ich mich } \\
\text { aktiv darum bemühen, eine Arbeitsstelle bei diesem } \\
\text { Unternehmen zu bekommen. }\end{array}$ & (1) & (2) & (3) & (4) & (5) \\
\hline POR4 & $\begin{array}{l}\text { Dieses Unternehmen erfüllt meine persönlichen Anfor- } \\
\text { derungen an einen Arbeitgeber. }\end{array}$ & (1) & (2) & (3) & (4) & (5) \\
\hline EWA1 & $\begin{array}{l}\text { Es ist wahrscheinlich, dass ich eine Stelle bei diesem } \\
\text { Arbeitgeber bekommen könnte. }\end{array}$ & (1) & (2) & (3) & (4) & (5) \\
\hline EWA2 & $\begin{array}{l}\text { Meine Bewerbung bei dem Unternehmen hätte Aus- } \\
\text { sichten auf Erfolg. }\end{array}$ & (1) & (2) & (3) & (4) & (5) \\
\hline POR3 & Dieses Unternehmen würde gut zu mir passen. & (1) & (2) & (3) & (4) & (5) \\
\hline
\end{tabular}


Stellen Sie sich nun bitte eine Person vor, deren Meinung Ihnen besonders wichtig ist. Schätzen Sie nun die folgenden Aussagen in Bezug auf das obige Stellenangebot ein.

\begin{tabular}{|c|c|c|c|c|c|c|}
\hline & & $\begin{array}{c}\text { stimme } \\
\text { überhaupt } \\
\text { nicht zu }\end{array}$ & $\begin{array}{c}\text { stimme } \\
\text { eher nicht } \\
\text { zu }\end{array}$ & weder noch & $\begin{array}{l}\text { stimme } \\
\text { eher zu }\end{array}$ & $\begin{array}{l}\text { stimme voll } \\
\text { und ganz zu }\end{array}$ \\
\hline SUB3 & $\begin{array}{l}\text { Sie wäre stolz auf mich, wenn ich für dieses Unter- } \\
\text { nehmen arbeiten würde. }\end{array}$ & (1) & (2) & (3) & (4) & (5) \\
\hline SUB1 & $\begin{array}{l}\text { Diese Person würde es begrüßen, wenn ich für dieses } \\
\text { Unternehmen tätig wäre. }\end{array}$ & (1) & (2) & (3) & (4) & (5) \\
\hline SUB2 & $\begin{array}{l}\text { Ich würde ihre Erwartungen erfüllen, wenn ich für } \\
\text { dieses Unternehmen arbeiten würde. }\end{array}$ & (1) & (2) & (3) & (4) & (5) \\
\hline & & $\begin{array}{c}\text { gar nicht } \\
\text { wichtig }\end{array}$ & \multicolumn{2}{|c|}{$\begin{array}{c}\text { eher } \\
\text { unwichtig }\end{array}$} & $\begin{array}{c}\text { eher } \\
\text { wichtig }\end{array}$ & $\begin{array}{c}\text { sehr } \\
\text { wichtig }\end{array}$ \\
\hline SUB4 & $\begin{array}{l}\text { Wie wichtig ist Ihnen die Meinung dieser Person } \\
\text { zu Ihrem Bewerbungsvorhaben bzw. zu Ihrer } \\
\text { Arbeitgeberwahl? }\end{array}$ & (1) & \multicolumn{2}{|c|}{ (2) } & (3) & (4) \\
\hline
\end{tabular}

Nun würden wir gern wissen, wie Sie sich über Ihre potenziellen Arbeitgeber informieren. Geben Sie bitte zunächst an, ob die Informationsquelle Ihnen bekannt ist, und im zweiten Schritt, ob Sie sich mindestens einmal persönlich mit ihr beschäftigt haben.

\begin{tabular}{|c|c|c|c|c|c|}
\hline \multirow{2}{*}{\multicolumn{2}{|c|}{ Die Informationsquelle... }} & \multicolumn{2}{|c|}{...ist mir bekannt } & \multicolumn{2}{|c|}{$\begin{array}{l}\text {...habe mich persönlich } \\
\text { damit beschäftigt }\end{array}$} \\
\hline & & ja & nein & ja & nein \\
\hline INP2 & Stellenanzeigen in Stellenbörsen im Internet & (1) & (2) & (1) & (2) \\
\hline INP1 & Stellenanzeigen in Zeitungen oder Zeitschriften & (1) & (2) & (1) & (2) \\
\hline INP9 & Job- und Fachmessen außerhalb der Hochschule & (1) & (2) & (1) & (2) \\
\hline INP7 & Fachvorträge von Unternehmensvertretern in Vorlesungen & (1) & (2) & (1) & (2) \\
\hline INP4 & Praktika/Werkstudententätigkeiten & (1) & (2) & (1) & (2) \\
\hline INP8 & Unternehmensexkursionen & (1) & (2) & (1) & (2) \\
\hline INP3 & $\begin{array}{l}\text { Unternehmenspräsentationen an Hochschulen (z.B. Un- } \\
\text { ternehmensmesse) }\end{array}$ & (1) & (2) & (1) & (2) \\
\hline INP6 & Kontakte zu Professoren relevanter Fachrichtungen & (1) & (2) & (1) & (2) \\
\hline INP10 & hochschuleigene Datenbanken & (1) & (2) & (1) & (2) \\
\hline INP12 & Soziale Netzwerke (z.B. Facebook) & (1) & (2) & (1) & (2) \\
\hline INP11 & Stellenangebote auf der Unternehmenshomepage & (1) & (2) & (1) & (2) \\
\hline INP5 & Unterstützung von Projekt- und Abschlussarbeiten & (1) & (2) & (1) & (2) \\
\hline INP13 & Karrierenetzwerke (z.B. XING) & (1) & (2) & (1) & (2) \\
\hline
\end{tabular}




\begin{tabular}{|c|c|c|c|c|c|c|}
\hline \multicolumn{2}{|c|}{ Arbeitgeberwahlstudie } & \multicolumn{5}{|c|}{$\begin{array}{l}\text { Hochschule Osnabrück } \\
\text { University of Applied Sclences }\end{array}$} \\
\hline & & $\begin{array}{c}\text { überhaupt } \\
\text { nicht } \\
\text { nützlich }\end{array}$ & $\begin{array}{c}\text { eher } \\
\text { unnützlich }\end{array}$ & teils teils & eher nützlich & $\begin{array}{l}\text { sehr } \\
\text { nützlich }\end{array}$ \\
\hline INP2 & Stellenanzeigen in Stellenbörsen im Internet & (1) & (2) & (3) & (4) & (5) \\
\hline INP1 & Stellenanzeigen in Zeitungen oder Zeitschriften & (1) & (2) & (3) & (4) & (5) \\
\hline INP9 & $\begin{array}{l}\text { Job- und Fachmessen außerhalb der Hochschule sind } \\
\text { nützlich und informativ }\end{array}$ & (1) & (2) & (3) & (4) & (5) \\
\hline INP7 & $\begin{array}{l}\text { Fachvorträge von Unternehmensvertretern in Vorle- } \\
\text { sungen }\end{array}$ & (1) & (2) & (3) & (4) & (5) \\
\hline INP4 & Praktika/Werkstudententätigkeiten & (1) & (2) & (3) & (4) & (5) \\
\hline INP8 & Unternehmensexkursionen & (1) & (2) & (3) & (4) & (5) \\
\hline INP3 & $\begin{array}{l}\text { Unternehmenspräsentationen an Hochschulen (z.B. } \\
\text { Unternehmensmesse) }\end{array}$ & (1) & (2) & (3) & (4) & (5) \\
\hline INP6 & Kontakte zu Professoren relevanter Fachrichtungen & (1) & (2) & (3) & (4) & (5) \\
\hline INP10 & hochschuleigene Datenbanken & (1) & (2) & (3) & (4) & (5) \\
\hline INP12 & Soziale Netzwerke (z.B. Facebook) & (1) & (2) & (3) & (4) & (5) \\
\hline INP11 & Stellenangebote auf der Unternehmenshomepage & (1) & (2) & (3) & (4) & (5) \\
\hline INP5 & Unterstützung von Projekt- und Abschlussarbeiten & (1) & (2) & (3) & (4) & (5) \\
\hline INP13 & Karrierenetzwerke (z.B. XING) & (1) & (2) & (3) & (4) & (5) \\
\hline
\end{tabular}

Nennen Sie bitte abschließend drei Informationsquellen aus der obigen Liste, die Sie für Ihre Arbeitgeberwahl für besonders informativ und nützlich erachten.

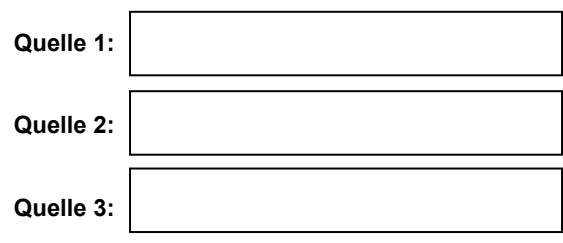

\section{Vielen Dank für Ihre Mitarbeit!}




\begin{tabular}{|c|c|c|c|c|c|c|c|c|c|c|c|c|c|c|c|c|}
\hline \multirow{2}{*}{$\hat{\hat{z}}$} & ठै & $\underset{\sim}{\stackrel{N}{r}}$ & $\begin{array}{l}\stackrel{R}{0} \\
0\end{array}$ & $\begin{array}{l}8 \\
0 \\
0\end{array}$ & $\begin{array}{l}5 \\
0 \\
0\end{array}$ & $\underset{0}{N}$ & 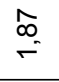 & $\begin{array}{l}\forall \\
0 \\
0\end{array}$ & $\underset{\sim}{\tilde{\sigma}}$ & $\begin{array}{l}\tilde{\delta} \\
\tilde{O}\end{array}$ & $\stackrel{m}{=}$ & 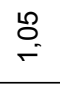 & \multirow{2}{*}{$\overline{\hat{\theta}}$} & \multirow{2}{*}{$\stackrel{\stackrel{2}{N}}{\underset{F}{F}}$} & \multirow{2}{*}{ ס̊ } & \\
\hline & 0 & $\begin{array}{l}\text { No } \\
0 \\
0\end{array}$ & $\begin{array}{l}\stackrel{ \pm}{N} \\
\stackrel{1}{1}\end{array}$ & $\begin{array}{c}\text { I } \\
\text { ì }\end{array}$ & $\begin{array}{l}0 \\
0 \\
0 \\
i\end{array}$ & $\begin{array}{l}\text { o } \\
\text { i. } \\
\text { in }\end{array}$ & $\begin{array}{l}\text { đ్ } \\
0\end{array}$ & $\begin{array}{l}\text { * } \\
\text { o. } \\
\text { i. }\end{array}$ & $\begin{array}{l}\text { No } \\
\text { O. }\end{array}$ & $\begin{array}{l}\infty \\
0 \\
i \\
\end{array}$ & $\stackrel{\mathrm{O}}{\stackrel{\circ}{0}}$ & $\begin{array}{l}20 \\
0 \\
0\end{array}$ & & & & \\
\hline \multirow{2}{*}{$\stackrel{\vec{Z}}{\stackrel{\Xi}{a}}$} & ๘ै & $\stackrel{\infty}{\sim}$ & $\begin{array}{l}\Delta \\
0 \\
0\end{array}$ & $\begin{array}{l}\infty \\
\infty \\
0\end{array}$ & $\begin{array}{l}\text { fo } \\
0\end{array}$ & $\stackrel{\infty}{\stackrel{\infty}{0}} \stackrel{0}{0}$ & $\stackrel{\hat{m}}{r}$ & $\begin{array}{l}R \\
\text { R }\end{array}$ & $\stackrel{\stackrel{\infty}{\sim}}{\sim}$ & $\stackrel{\circ}{\stackrel{\circ}{-}}$ & 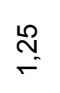 & $\underset{\sim}{\stackrel{\Xi}{-}}$ & \multirow{2}{*}{$\overline{\hat{\theta}}$} & \multirow{2}{*}{ 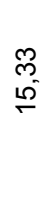 } & \multirow{2}{*}{$\begin{array}{l}8 \\
0\end{array}$} & \\
\hline & - & 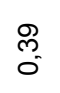 & 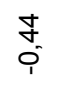 & $\frac{m}{\varphi^{\prime}}$ & $\begin{array}{l}R \\
0 \\
i\end{array}$ & $\begin{array}{l}m \\
\stackrel{0}{1} \\
i\end{array}$ & $\bar{g}_{0}$ & $\begin{array}{l}0 \\
\tilde{m} \\
i\end{array}$ & 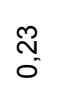 & $\frac{\pi}{0}$ & $\begin{array}{l}\text { Nָ} \\
0\end{array}$ & $\begin{array}{l}\text { O } \\
0 \\
0\end{array}$ & & & & \\
\hline \multirow{2}{*}{$\hat{\underline{z}}$} & ठै & $\begin{array}{l}\text { Jे } \\
\text { O }\end{array}$ & $\begin{array}{l}\text { đo } \\
0\end{array}$ & $\stackrel{\stackrel{\infty}{\sim}}{\sim}$ & $\underset{-}{\stackrel{8}{-}}$ & 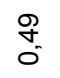 & $\stackrel{\infty}{\stackrel{\infty}{\leftarrow}}$ & $\begin{array}{l}\infty \\
\stackrel{\infty}{\infty} \\
0\end{array}$ & $\stackrel{\leftrightarrow}{\stackrel{-}{-}}$ & $\begin{array}{l}\tilde{O} \\
\delta \\
O\end{array}$ & $\underset{\sim}{\stackrel{\sigma}{r}}$ & $\stackrel{\infty}{\stackrel{\infty}{\leftarrow}}$ & \multirow{2}{*}{$\overleftarrow{\hat{\imath}}$} & \multirow{2}{*}{ 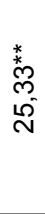 } & \multirow{2}{*}{$\stackrel{8}{0}$} & \\
\hline & - & $\begin{array}{l}8 \\
\stackrel{-}{1}\end{array}$ & $\begin{array}{l}\infty \\
\stackrel{+}{i} \\
i\end{array}$ & $\stackrel{\stackrel{2}{N}}{\stackrel{0}{0}}$ & O & $\begin{array}{l}\overline{\hat{T}} \\
\bar{i}\end{array}$ & $\begin{array}{c}\text { ले } \\
\text { o. }\end{array}$ & 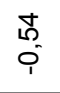 & $\begin{array}{l}8 \\
0\end{array}$ & $\begin{array}{l}\infty \\
0 \\
i^{-1}\end{array}$ & $\begin{array}{l}\tilde{O} \\
0 \\
0\end{array}$ & $\begin{array}{l}0 \\
0 \\
0\end{array}$ & & & & \\
\hline \multirow{2}{*}{$\tilde{\tilde{z}}$} & ๘ & $\begin{array}{l}\text { g } \\
\text { o. }\end{array}$ & $\stackrel{\leftrightarrow}{\circ}$ & $\begin{array}{l}\text { న్ } \\
\text { ల) }\end{array}$ & 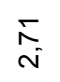 & $\begin{array}{l}\text { ठ } \\
\text { o }\end{array}$ & $\stackrel{\stackrel{\infty}{\sim}}{\sim}$ & $\begin{array}{l}0 \\
0 \\
0\end{array}$ & $\underset{F}{\check{F}}$ & 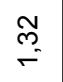 & $\begin{array}{l}\Sigma \\
0 \\
0\end{array}$ & 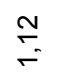 & \multirow{2}{*}{$\overline{\hat{\theta}}$} & \multirow{2}{*}{$\begin{array}{l}\stackrel{N}{N} \\
\sigma^{\prime}\end{array}$} & \multirow{2}{*}{$\begin{array}{l}\hat{0} \\
0\end{array}$} & \\
\hline & 0 & $\begin{array}{l}\overline{0} \\
0 \\
\end{array}$ & $\begin{array}{l}\text { స్ } \\
0 \\
0\end{array}$ & $\stackrel{\hat{m}}{=}$ & $\stackrel{8}{\circ}$ & $\begin{array}{l}0 \\
0 \\
i\end{array}$ & 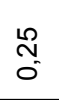 & $\begin{array}{l}\text { fo } \\
\stackrel{i}{i}\end{array}$ & $\frac{0}{0}$ & $\begin{array}{l}\hat{N} \\
\tilde{0}\end{array}$ & $\begin{array}{l}\stackrel{0}{0} \\
\stackrel{m}{1}\end{array}$ & $\underset{0}{E}$ & & & & \\
\hline \multirow{3}{*}{$\overline{\bar{z}}$} & ठै & $\underset{\mathbb{N}}{\sim}$ & $\stackrel{\Gamma}{r}$ & $\begin{array}{c}\stackrel{2}{0} \\
\stackrel{0}{0}\end{array}$ & $\begin{array}{l}8 \\
0\end{array}$ & $\begin{array}{l}8 \\
0\end{array}$ & $\begin{array}{l}8 \\
8 \\
0\end{array}$ & $\underset{⿱ 亠}{\sim}$ & $\stackrel{\infty}{\stackrel{\infty}{\sim}}$ & 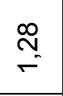 & $\begin{array}{l}\bar{\delta} \\
\text { o. }\end{array}$ & 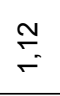 & \multirow{2}{*}{$\overline{0}$} & \multirow{2}{*}{$\begin{array}{l}\stackrel{8}{\infty} \\
\stackrel{\sim}{\sim}\end{array}$} & \multirow{2}{*}{$\begin{array}{l}8 \\
0 \\
0\end{array}$} & \\
\hline & 2 & ָָ & $\begin{array}{l}\text { 芯 } \\
0\end{array}$ & $\begin{array}{l}\text { N } \\
\text { ô }\end{array}$ & $\frac{\stackrel{8}{f}}{\stackrel{5}{\leftarrow}}$ & 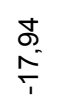 & $\begin{array}{l}\text { ¿ } \\
\text { ì }\end{array}$ & $\begin{array}{l}\bar{\infty} \\
0 \\
0\end{array}$ & 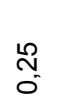 & $\begin{array}{l}\stackrel{L}{N} \\
\text { ô }\end{array}$ & $\begin{array}{l}8 \\
0 \\
0 \\
1\end{array}$ & $\stackrel{N}{\check{0}}$ & & & & $\begin{array}{l}0 \\
v \\
2 \\
* \\
*\end{array}$ \\
\hline & 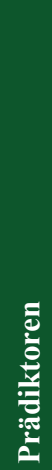 & 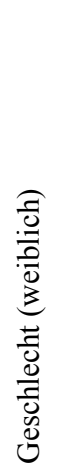 & 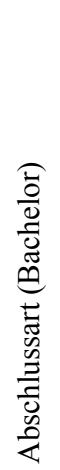 & 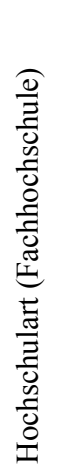 & 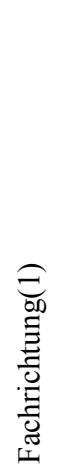 & 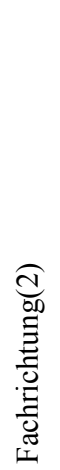 & 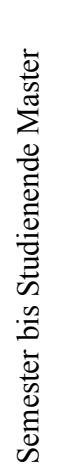 & 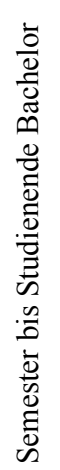 & 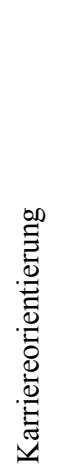 & 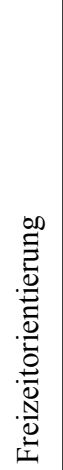 & 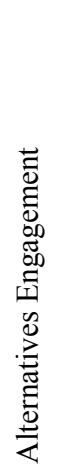 & 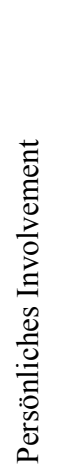 & z & 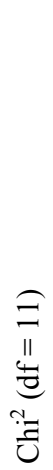 & 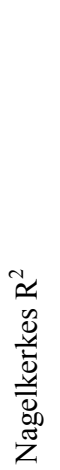 & 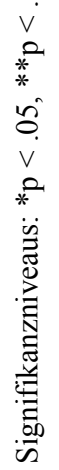 \\
\hline
\end{tabular}




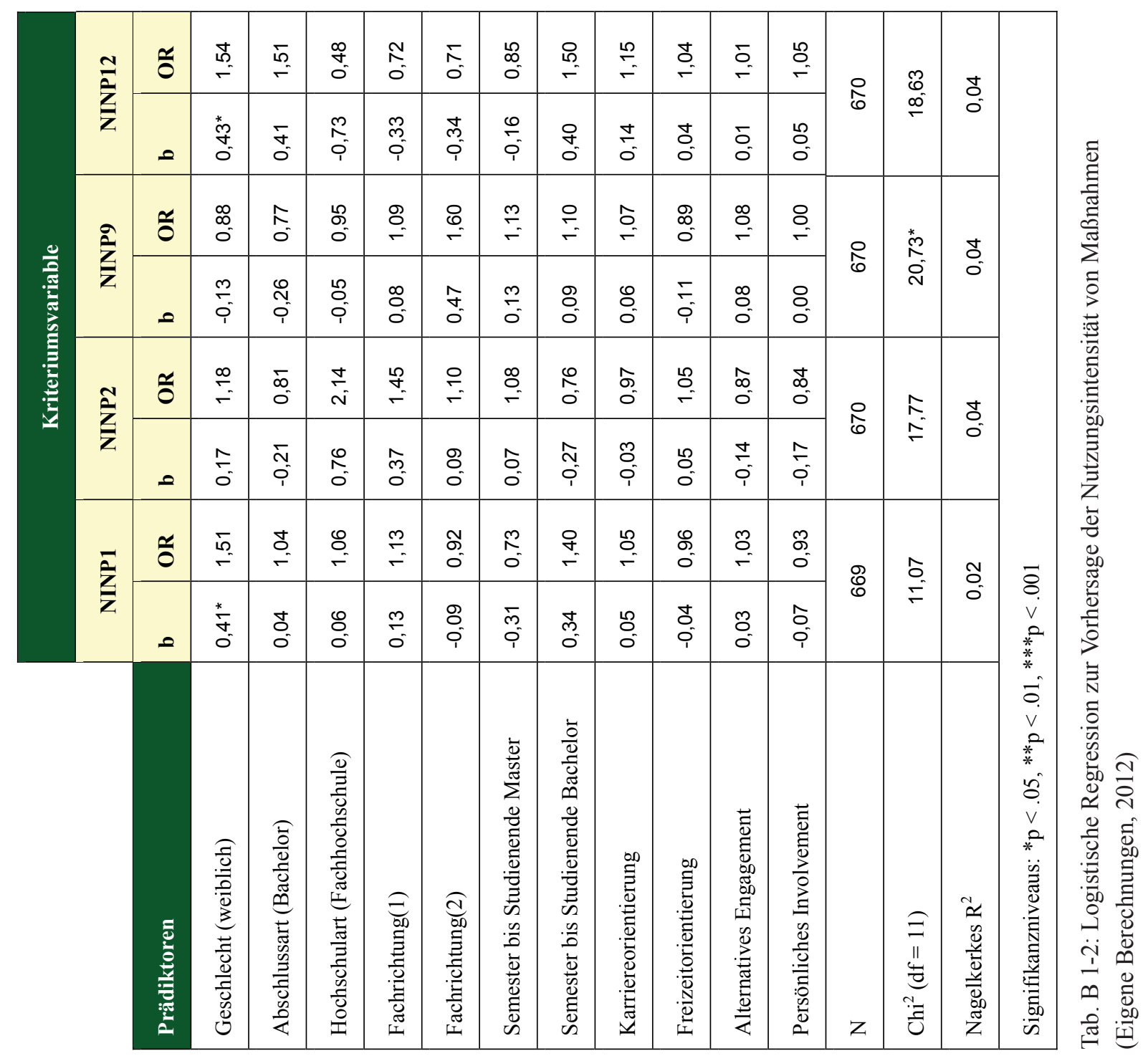




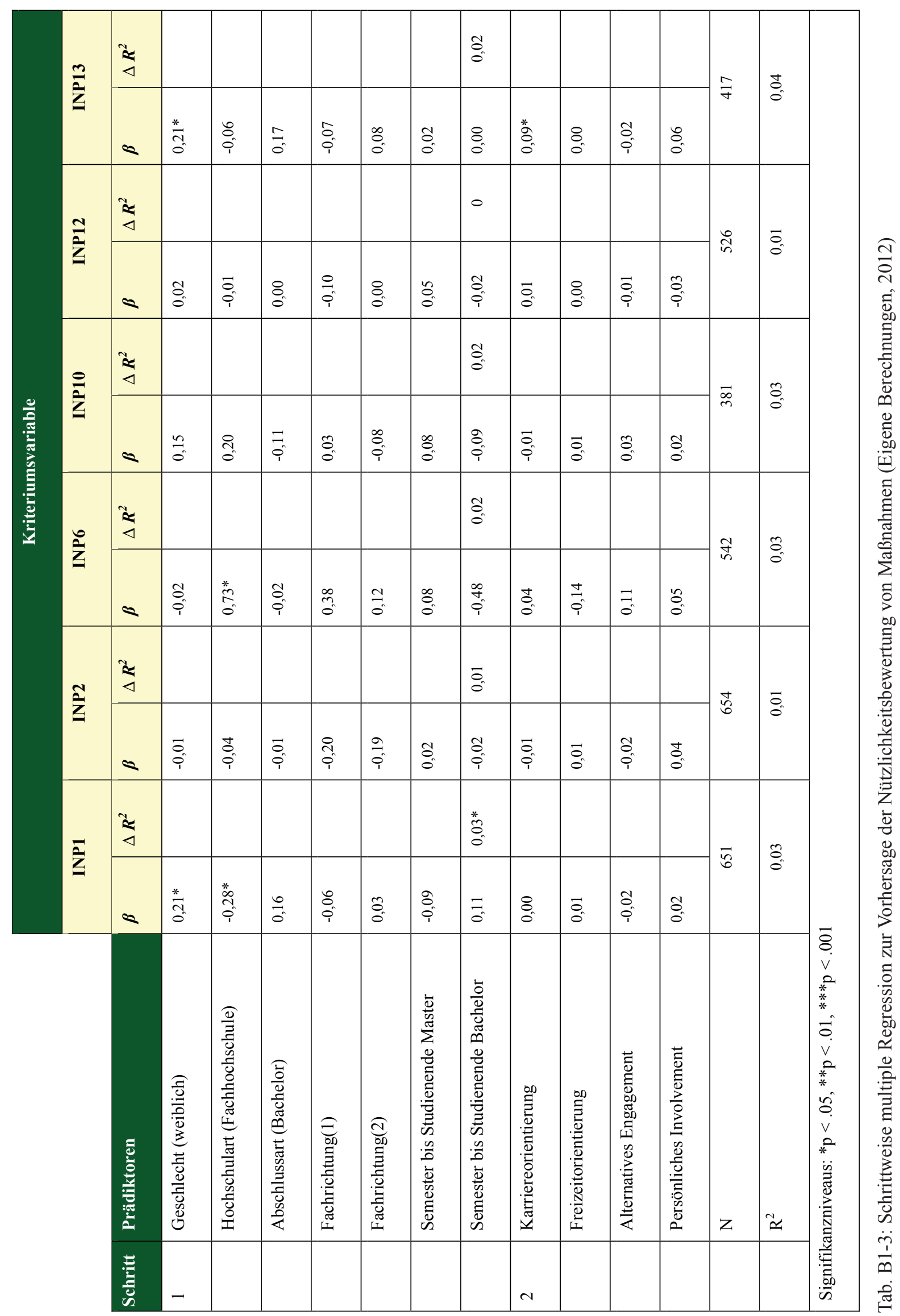

